Facoltà di Science Matematiche, Fisiche e Naturali

Dipartimento di Scienze Biomolecolari e Biotecnologie

Scuola di dottorato in Scienze Biologiche e Molecolari Corso di Dottorato di Ricerca in Biologia Cellulare e Molecolare Ciclo XXI

\title{
Functional analysis and localization studies of Phr1 and Gas1 proteins from the fungal pathogen Candida albicans and the budding yeast Saccharamyces cerevisiae
}

Tesi di dottorato di:

JULIA CALDERÓN BLANCO (matricola $\mathrm{n}^{\mathrm{O}}$ : R06755)

Relatore: Prof.ssa Laura Popolo

Coordinatore del dottorato: Prof.ssa Giuliana Zanetti 


\section{FUNDING:}

This work has been supported by the "CanTrain" EU Research Training Network CT2004-512481. I was a recipient of a Marie Curie fellowship from 2006 to 2009. 


\section{ACKNOWLEDGMENTS}

I would like to express my deepest gratitude to my supervisor, Prf.ssa Laura Popolo who was extremely helpful and offered invaluable assistance, support and guidance. I would like to thanks the other members of the group, Eleonora Rolli who was my $\mathrm{PhD}$ colleague during the whole period of my work in Italy and Enrico Ragni.

I also would like to thanks Dott. Umberto Fascio from C.I.M.A (Centro Interdisciplinare di Microscopia Avanzata) from the University of Milan.

I wish to adknowledge my Thesis Committee: Prf.ssa Giulia Morace and Prof. Marco Muzzifalcone from University of Milan.

I would like to thanks to the members of the Cantrain Project (EU Research Training Network) and specially to Prof. Patrick Van Dijck (project coordinator) from the VIB Department of Molecular Microbiology, K.U., Leuven (Belgium).

I also would like to thanks Prof. Steffen Rupp from the Fraunhofer Institute (FHG), in Stuttgart (Germany) for the opportunity to work in his group

I wish to express my gratitude also to my mentor in Spain, Prof. Dr. Ángel Domínguez Olavarri from the University of Salamanca (Spain).

Many thanks to my friends. For those that I have meet in Italy and those who have been always near me (even in the distance).

Special thanks goes to my family for the support they provided me through my entire life. Mi más sincero agradecimiento para vosotros, mi familia 


\section{INDEX}

INTRODUCTION.....................................................................

1. Candida albicans AS AN IMPORTANT PATHOGENIC FUNGUS .................1

1.1. Taxonomy and genomics........................................

1.2. Pathogenesis and therapy...........................................2

1.3. Virulence factors.................................................

1.3.1. Phenotypic switching ......................................4

1.3.2. Morphological dimorphism....................................5

1.3.3. Adherence and adherence molecules...........................6

1.3.4. Secreted hydrolytic enzymes................................6

1.3.5. Other virulence factors...............................................

1.4. C. albicans lyfe-cycle and polymorphism...............................

1.4.1. Morphogenesis and role in virulence..........................8

1.4.2. Transcriptional control of morphogenesis.......................10

1.4.3. Morphogenesis checkpoint and cell cycle progression................13

1. 5. Host-pathogen interactions ...................................... 18

1.5.1. Adhesion to host tissues......................................18

1.5.2. Invasion process in Candida albicans............................19

2. THE FUNGAL CELL WALL............................................... 21

2.1. General overview ...............................................21

2.2. Structure end composition of the fungal cell wall........................21

2.2.1. $\beta$-1,3-glucan network.....................................22

2.2.2. $\beta-1,6$-glucan...........................................24

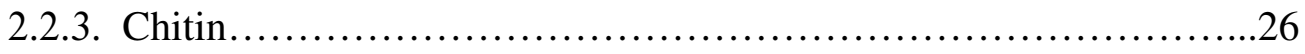

2.2.4. Cell wall proteins ..........................................29

3. THE GH72 FAMILY IN S. cerevisiae AND C. albicans..............................

3.1. GAS multigen family of $S$. cerevisiae................................ 31

3.1.1. Gas $1 \Delta$ mutant phenotype..................................34

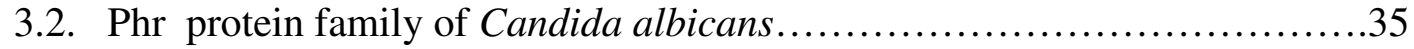

3.2.1. PHR1 deletion phenotype and its role in virulence...................36

3.2.2. The $\mathrm{pH}$ response in Candida albicans................................... 37 


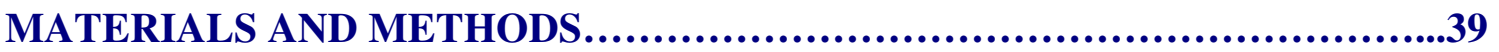

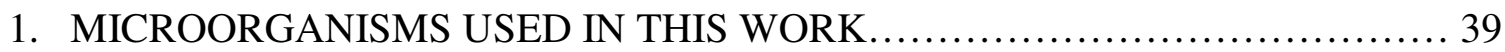

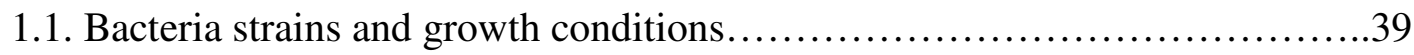

1.2. Saccharomyces cerevisiae strains and growth conditions.....................39

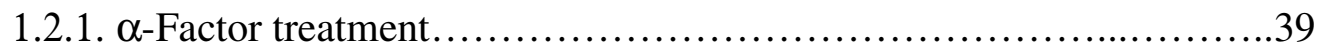

1.3. C. albicans strains and growth conditions used in this work.....................39

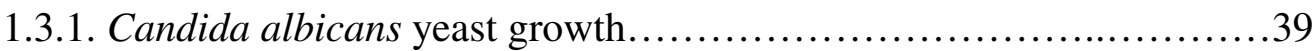

1.3.2. Induction of Candida albicans filamentation growth....................40

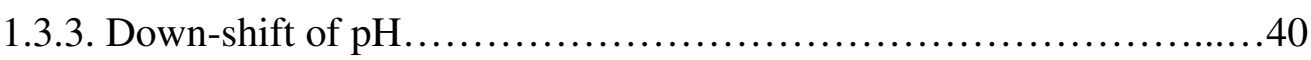

2. DNA MANIPULATION TECHNIQUES........................................ 43

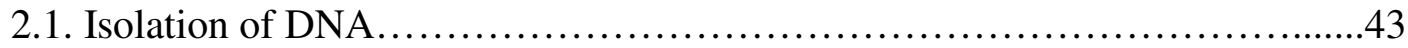

2.1.1. Preparation of plasmid DNA .....................................43

2.1.2. Total DNA extraction from yeast.....................................43

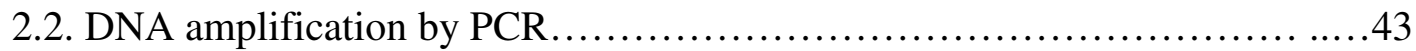

2.3. DNA Gel electrophoresis..................................................44

2.4. Purification of PCR products.............................................. 44

2.5. Isolation of DNA fragments from agarose gels........................... 45

3. TRANSFORMATION OF BACTERIA AND YEAST WITH

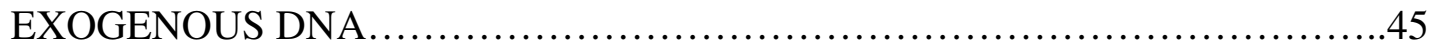

3.1. E. coli transformation.....................................................45

3.2. Saccharomyces cerevisiae transformation....................................46

3.3. Candida albicans transformation protocol......................................46

4. CONSTRUCTION OF S. cerevisiae AND C. albicans FUSION PROTEINS..........47

4.1. Construction of $S$. cerevisiae strain expressing mRFP-GAS1 fusion protein....47

4.2. Construction of $C$. albicans strain expressing Phr1-GFP fusion protein........47

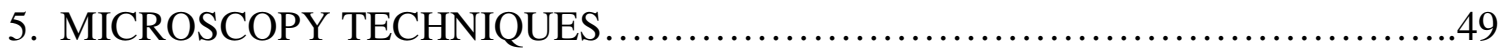

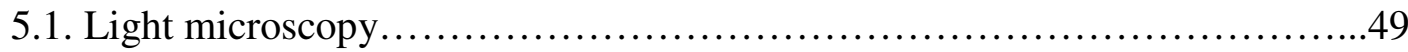

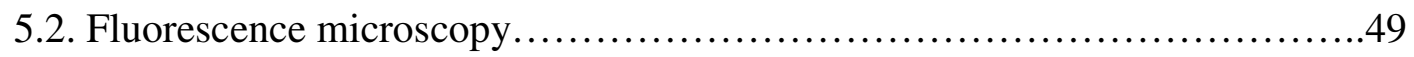

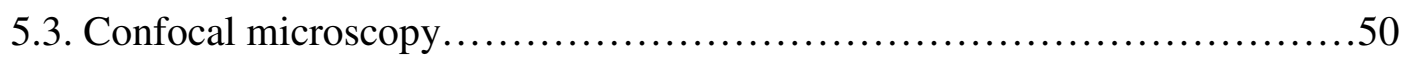

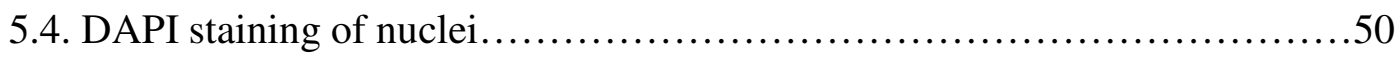

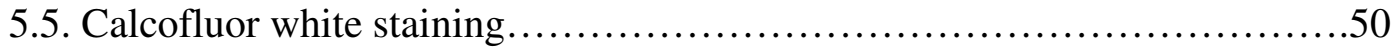

5.6. Rhodamine phalloidin staining ...........................................

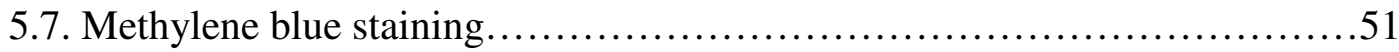


6. CALCOFLUOR WHITE SENSITIVITY ASSAY

7. TREATMENT OF C. albicans CELLS WITH Nocodazole (NZ)

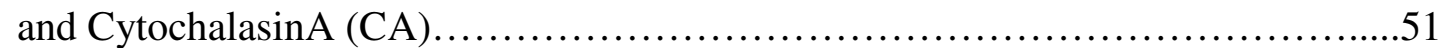

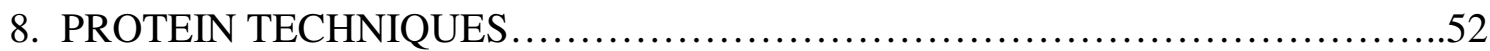

8.1. Total protein extracts from yeast...................................52

8.2. Subcellular fractionation and isolation of the cell walls....................53

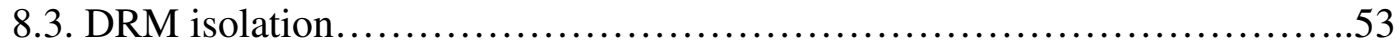

8.4. SDS-PAGE and immunoblotting ....................................5 54

9. ADHESION AND INVASION ASSAYS IN Candida albicans .....................54

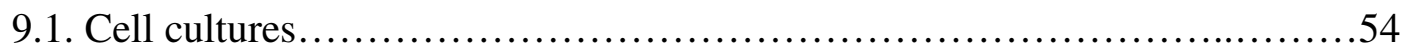

9.2. Adhesion assays................................................ 55

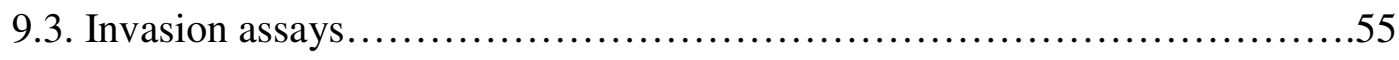

10. Quantification of mRNA using Real Time Quantitative reverse transcriptase PCR (qRT-PCR)....

OBJECTIVES

RESULTS

CHAPTER I................................................................59

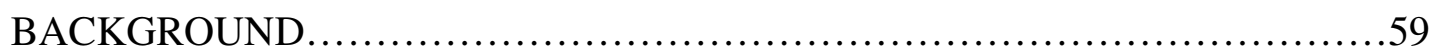

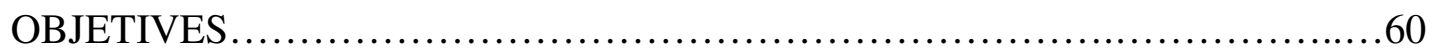

RESULTS .............................................................61

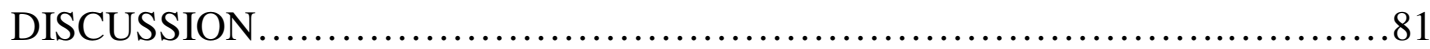

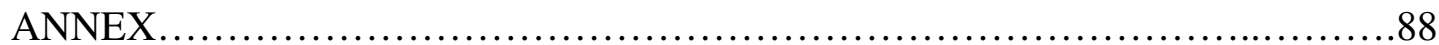

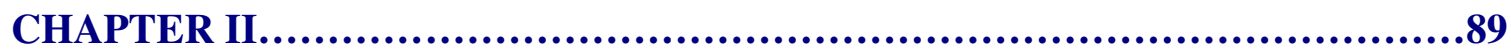

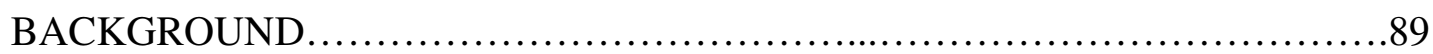

OBJETIVES ......................................................... 90

RESULTS........................................................... 90

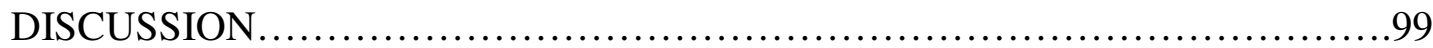

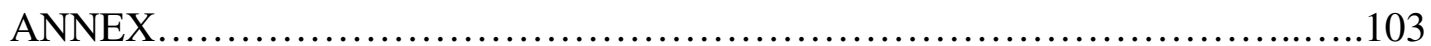

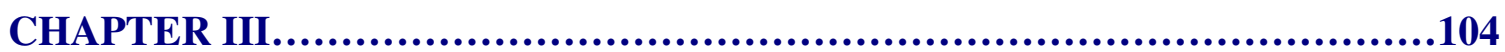

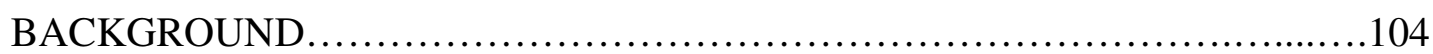

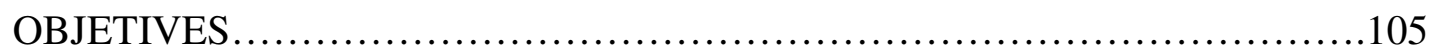


RESULTS. .106

DISCUSSION.

ANNEX.

CONCLUSIONS

\section{RESUMEN EN ESPAÑOL}

INTRODUCCIÓN............................................................124

OBJETIVOS......................................................................136

RESULTADOS

CAPÍTULO I........................................................................137

ANTECEDENTES ...................................................... 137

OBJETIVOS......................................................... 137

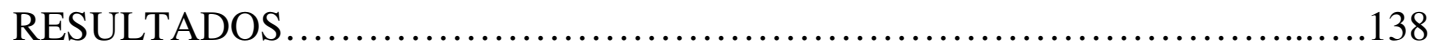

DISCUSIÓN ............................................................... 143

CAPÍTULO II........................................................................146

ANTECEDENTES ..................................................... 146

OBJETIVOS ........................................................... 147

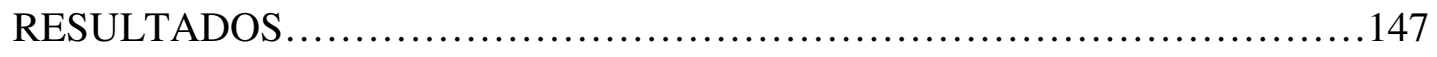

DISCUSIÓN ......................................................... 150

CAPÍTULO III......................................................................153

ANTECEDENTES .................................................. 153

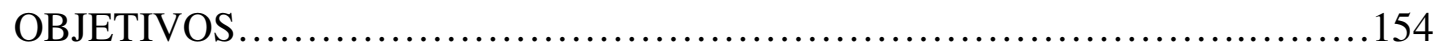

RESULTADOS ....................................................... 154

DISCUSIÓN................................................................

CONCLUSIONES......................................................................161

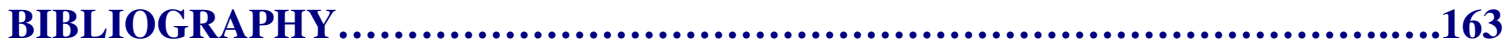


1. Candida albicans AS AN IMPORTANT PATHOGENIC FUNGUS:

\subsection{TAXONOMY AND GENOMICS:}

Candida albicans is a diploid fungus belonging to the Phylum Ascomycota.

Kingdom: Fungi

Phylum: Ascomycota

Subphylum: Ascomycotina

Class: Ascomycetes

Order: Saccharomycetales

Family: Saccharomycetaceae

Genus: Candida

Candida albicans is a diploid fungus that has become a medically relevant opportunistic pathogen in immunocompromised individuals. $C$. albicans is a morphologically complex organism capable of proliferating either as a budding yeast or by the formation of pseudohyphae or filamentous hyphae. C.albicans morphological complexity and diploidity, together with the inability to manifest a sexual cycle has significantly impeded conventional genetic analysis. Until recently, in fact, C. albicans was thought to be asexual, existing only as an obligate diploid. However, a mating locus and a genetic repertoire that could support a complete sexual cycle has been subsequently demonstrated in laboratory (Bennett and Johnson 2005) C. albicans has a diploid genome that is split between eight pairs of chromosomes that can be separated by pulse-field gel eletrophoresis. At $\sim 16 \mathrm{Mb}$, the haploid genome is slightly larger than that of $S$. cerevisiae, perhaps because of the greater number of retrotransposon families. It contains several large families of genes that encode for proteases, lipases and cell-wall proteins that are not present with a similar redundancy in the $S$. cerevisiae genome (Berman and Sudbery 2002).

The $C$. albicans genome was sequence by the Stanford Genome Technology Center, resulting in the release of successive assemblies. The completed and annotated sequence has been published in 2004 (Jones, Federspiel et al. 2004). Candida DB, a database dedicated to the analysis of the genome of the human fungal pathogen Candida albicans, avaible at: http://genolist.pasteur.fr/CandidaDB, has also been created. CandidaDB contains information pertaining to Assembly 19 of the genome of $C$. albicans strain SC5314. (d'Enfert, Goyard et al. 2005). Recently, supercontigs from Assembly 19 (183, representing $98.4 \%$ of the sequence) were assigned to individual 
chromosomes (van het Hoog, Rast et al. 2007)

\subsection{PATHOGENESIS}

AND

\section{THERAPY:}

The frequency of severe systemic fungal diseases has increased in the last few decades. Several factors have contributed. Among them are the expansion of the severely ill and/or immunocompromised population, including HIV-infected patients, patients with cancer who suffer of chemotherapyinduced neutropenia and transplant recipients who are receiving immunosuppressive therapy. Additional factors that have also contributed are the frequent use of more-invasive medical procedures, prolonged treatments with broad-spectrum antibiotics and glucocorticoids, receipt of parenteral nutrition, peritoneal dialysis or hemodialysis.

The most frequently diagnosed fungal infections are caused by pathogens from the genera Candida, Cryptococcus and Aspergillus (Richardson 2005). These fungi are ubiquitous and can be acquired from host surroundings (Cryptococcus neoformans, Aspergillus fumigatus) or are components of normal endogenous flora (Candida albicans) (KarkowskaKuleta, Rapala-Kozik et al. 2009). Nowadays, Candida albicans is thought to be the major fungal pathogen of humans. Severe Candida infections are a serious problem, especially in individuals whose immune defence mechanism has been weakened.

Candida infections (candidiasis) represent a major clinical problem with increasing incidence during the last years. Data from a study performed to evaluate contemporary epidemiology and outcomes of candidemia in multiple North American centres from 1 July 2004 through 5 March 2008 indicate that Candidemia (invasive candidiasis) remains associated with high crude and atributable mortality rates and with increased costs of care and duration of hospitalization. Attributable mortality has been reported to range from $5 \%$ to $71 \%$, and crude mortality rates have been reported to be as high as $81 \%$ (Horn, Neofytos et al. 2009).

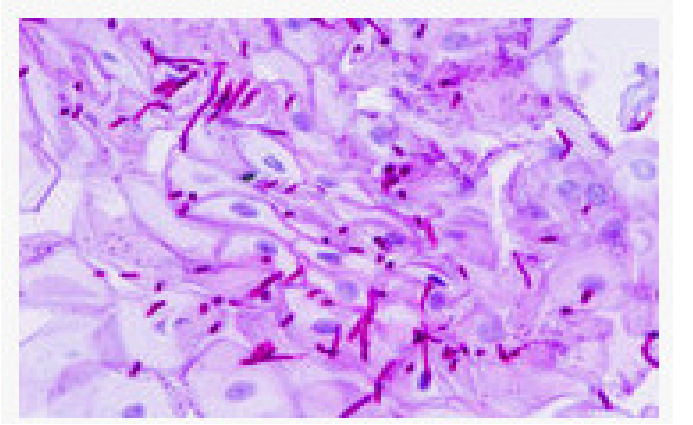

Figure 1. Esophageal candidiasis stained by periodic acid-Schiff procedure

C. albicans can colonize skin and mucosal surfaces of healthy people and thus occurs commensally in the gastrointestinal tract, oral cavity and 
vagina, often causing superficial infections (Mavor, Thewes et al. 2005) Moreover, $C$. albicans can enter the bloodstream by direct penetration from the epithelium after tissue damage, or by dissemination from biofilms formed on medical devices introduced into the patient's organism, e.g. catheters, dental implants, endoprotheses, artificial joints or central nervous system shunts. Then yeast cells disseminate with the blood flow and infect almost all inner organs, including lungs, kidney, heart, liver, spleen and brain, causing fungaemia and life-threatening septicaemia (Chandra, Kuhn et al. 2001; Mavor, Thewes et al. 2005; Karkowska-Kuleta, Rapala-Kozik et al. 2009).

It is important to consider that different recent data suggest that the epidemiology of invasive fungal infections is changing. The vast majority of the invasive fungal infections are still due to Aspergillus and Candida species, but infections due to mycelial fungi other than Aspergillus and to non albicans species of Candida are increasingly common (Fluckiger, Marchetti et al. 2006; Asmundsdottir, Erlendsdottir et al. 2008). In this context, the choice of an appropriate therapy is becoming more and more difficult.

The treatment of fungal infections, especially for the systemic infections, is difficult due to the genetic similarity between host and pathogen, both eukaryotes. Other factors should be considered. The appropriate antifungal therapy and selection of drug should be based on criteria such as: immune status of the host, site of infection, characteristics of the infection (the fungal species and its susceptibility to different antifungal drugs) and pharmacokinetic characteristics of the drug (e.g., absoption, elimination, and toxicity). Only a limited number of antimycotic drugs are available for the treatment of systemic fungal infections. On the basis of the mode of action the most important antifungal drugs can be divided into 4 different classes: (1) polyene macrolides that lead to an alteration of membrane fuctions (amphotericin A and its lipid formulations); (2) azole derivates that inhibit the $14 \alpha$-lanosterol demethylase, a key enzyme in ergosterol biosynthesis (ketonazole , fluconazole, itraconazolea and voriconazole); (3) DNA and RNA synthesis inhibitors (flucytosine); and (4) 1,3- $\beta$-glucan synthase inhibitors (echinochandines). Although new drugs have been introduced during the last years to combat invasive fungal infections, the development of resistance to antifungal drugs has become increasingly apparent, especially in 
patients who require long-term

treatments or are receiving antifungal prophylaxis and there is a growing awareness of shifts of the flora to moreresistance species.

Recently the development of a number of antifungals with increased potency and lower toxicity, have raised optimism that outcomes for invasive fungal infection can be improved upon. The availability of lipid formulations of amphotericin B, azoles with extended spectrum against filamentous fungi and the development of a new class of antifungal agents, the echinocandins, presents the clinician with a range of therapeutic choices. Recent clinical trials have provided important insights into how these agents should be used (Metcalf and Dockrell 2007).

Fortunately, during the last few years, virulence factors of fungi and their inhibitors have, at least to some extent, been discovered and characterized. This should provide new options for the development of potential antifungal therapeutics. Nevertheless, many therapeutic areas of uncertainty remain, including the role of combination therapy, and will provide the focus for future studies (Gauwerky, Borelli et al. 2009).

\subsection{VIRULENCE FACTORS:}

Candidiasis may occur as a result of disturbed balance between host immunity and the commensal microorganism. This disorder is not only due to the immunological dysfunction of the host, but also to the fungal ability to adapt to new niches, a feature which depends on the expression of infectionassociated genes (Brown, Odds et al. 2007). These genes and their products contribute to fungal pathogenicity and are described as virulence factors. $C$. albicans virulence factors include, among others the production of different hydrolytic enzymes and adhesins (Chaffin, Lopez-Ribot et al. 1998). There are also other characteristics that influence fungal virulence, for example, the ability to form biofilms on various surfaces, to change morphology and to switch between various phenotypes (Chaffin, Lopez-Ribot et al. 1998)

\subsubsection{Phenotypic switching:}

Occasionally some subpopulations of C. albicans cells can change their morphology, cell surface properties, colony appearance, biochemical properties and metabolism to become more virulent and more effective during infection (Karkowska-Kuleta, RapalaKozik et al. 2009). The most popular and well-known example of switched 
colonies is the white-opaque switching, when a white, oval and smooth colony changes into a grey, rough colony. It has been demonstrated that a variety of virulence characteristics differ between cells in the two phases.

While opaque-phase cells secrete high levels of aspartyl proteases, white-phase cells do not; while white-phase cells differentially express the drug-resistance genes CDR4, opaque-phase cells differentially express the drug-resistance gene CDR3 (Soll 2004).
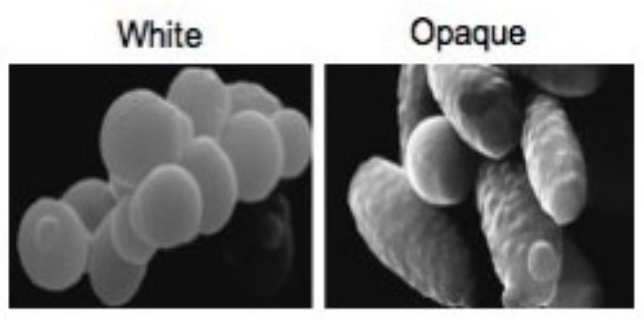

Figure 2. Scanning electron micrographs of white and opaque colonies of Candida albicans

The opaque cells produce aspartyl proteinases 1 and 3 and are less virulent, whereas white cells secrete aspartyl proteinase 2 and are more virulent during systemic infection. Phenotypic switching is most likely a signal of largescale processes involving changes of many molecular and biochemical properties of the pathogen, which are helpful for fungi to survive within the host organism (Karkowska-Kuleta, Rapala-Kozik et al. 2009).
1.3.2. Morphological dimorphism:

C. albicans is able to reversibly change its morphology from budding cells to elongated hyphae or filamentous growth forms. This morphological plasticity appears to be a key contributor to virulence. Many conditions induce filamentous growth. Among these, alterations in $\mathrm{pH}$, moderately high temperature, nutrient deprivation, and addition of serum or $\mathrm{N}$ acetylglucosamine (GlcNAc) are the most commonly used conditions (Sanchez-Martinez and Perez-Martin 2001). Yeast cells are thought to be responsible for dissemination in the environment and finding new hosts, while hyphae are required for tissue damage and invasion.
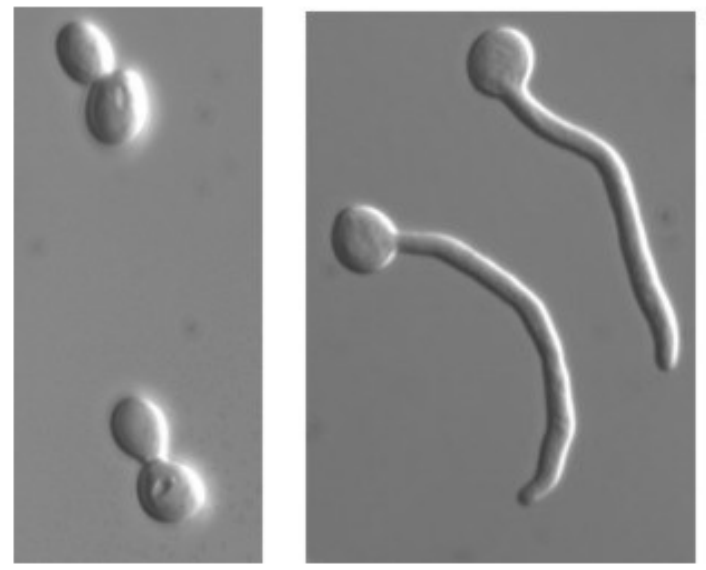

Figure 3. DIC images of Candida albicans growing as yeast (left) and hyphae (right).

Both forms are present in biofilms formed on artificial substrates (Chandra, Kuhn et al. 2001). Yeast cells have different properties than the mycelial 
forms: the ultrastructure, biological attributes and composition of the cell wall differ between these forms. Probably both forms, yeast cells and hyphae, are necessary for full virulence, because mutants lacking genes responsible for the production of one or the other are less virulent (Yang 2003).

Role of morphological dimorphism in the pathogenesis of $C$. albicans will be analyzed later on with more detail.

\subsubsection{Adherence and adherence} molecules:

The adherence to the host cells and tissues, as well as the binding to a set of diverse host proteins is essential for $C$. albicans to begin the invasion, followed by dissemination within the human organism. This step is crucial for fungal survival. On the cell wall surface $C$. albicans presents receptors which are responsible for adhesion to epithelial and endothelial cells, serum proteins and extracellular matrix proteins (Chaffin, Lopez-Ribot et al. 1998). Adhesion to different artificial substrates and formation of biofilms on medical devices is currently a serious problem in medicine, because of the frequent resistance to antifungal agents and increased pathogenicity among the subpopulation of cells forming the biofilm. During biofilm formation, $C$. albicans cells express several genes that influence pathogenicity. Products of these genes take part in adhesion (e.g. family of Als proteins), in carbohydrate synthesis, drug resistance (e.g. efflux pumps) and in quorum sensing (Chandra et al., 2001).

Adhesion in Candida albicans will be treated later on in the frame of hostpathogen interactions.

\subsubsection{Secreted hydrolytic enzymes:}

Production and secretion of hydrolytic enzymes, such as proteases, lipases and phospholipases are very important virulence factors. These enzymes play a role in fungal nutrition but also in tissue damage, dissemination within the human body, iron acquisition and overcoming the host immune system, and their orchestrated action strongly contributes to fungal pathogenicity. Many types of secreted hydrolytic enzymes are currently known for C. albicans (Yang 2003).

The activity of phospholipases is very high during tissue invasion, because these enzymes are responsible for hydrolysis of one or more ester linkages of glycerophospholipids, which are important constituents of cell membranes. $C$. albicans cells isolated from blood produce higher extracellular phospholipase activities compared with 
commensal strains. There are four types of secreted phospholipases: A, B, C and D (Yang 2003). The activity of phospholipase B (PLB), which has both hydrolase and lysophospholipasetransacylase activities, is very important for fungal virulence. Apart from phospholipases, C. albicans can produce at least nine lipases which can hydrolyze ester bonds of mono-, di- and triacylglycerols. A well-known group of C. albicans secreted hydrolytic enzymes are SAPs (secreted aspartyl proteinases). The family of $S A P$ genes includes at least ten different genes SAP1-SAP10 which encode enzymes with similar functions, but different molecular properties, such as molecular mass, isoelectric point and $\mathrm{pH}$ for optimal activity (Naglik, Fostira et al. 2006). Probably SAPs 1-3 are secreted only by yeast cells and SAPs 4-6 by hyphal forms, whereas both forms produce SAPs 9 and 10, which are connected with fungal cell walls because the presence of a GPI attachment signal. The synthesis and function of SAPs 7 and 8 are still under investigation. Many host proteins are hydrolyzed by secreted aspartyl proteinases, including collagen, laminin, fibronectin, mucin, salivary lactoferrin, $\alpha 2$-macroglobulin, almost all immunoglobulins, the proinflammatory cytokine interleukin-1 $\beta$, lactoperoxidase, cathepsin D, complement, cystatine A and precursors of several blood coagulation factors. The spectrum of optimal $\mathrm{pH}$ for SAPs activity is from 2.0 to 7.0 , therefore these enzymes may contribute to fungal pathogenesis and developing infections in different sites in the human body (Naglik, Challacombe et al. 2003; Naglik, Albrecht et al. 2004)

\subsubsection{Other virulence factors:}

The ability of pathogenic microorganisms to acquire iron from the environment during infection is another very important virulence factor. The ability to overcome host systems connected with iron transport and accumulation is crucial for the pathogen to survive during invasion of the bloodstream. In C. albicans Rbt5 protein has been shown to be involved in the utilization of hemoglobin and hemin for iron acquisition by the pathogen. Without this protein the C. albicans iron metabolism is severely impaired (Weissman and Kornitzer 2004). During infection, Candida cells are exposed to reactive oxygen species produced by immune cells, hence the organism expresses several virulence factors which help to overcome this host defense mechanism, including catalase, superoxide dismutase and heat shock proteins . Expression of many virulence 
factors often depends on environmental conditions, therefore fungi must possess a se (Brown, Odds et al. 2007). Expression of many virulence factors often depends on environmental conditions; therefore fungi must possess sensor/s for environmental changes. Probably calcineurin is one of the keyplayer to act as a sensor. Calcineurin is dispensable for growth at $37^{\circ} \mathrm{C}$, germ tube formation, and adherence to the host cells, but is essential for survival in the human serum, so fungal pathogenicity strongly correlates with its activity. It has been shown that calcineurin may influence the expression of several virulence factors of $C$. albicans (Karkowska-Kuleta, RapalaKozik et al. 2009). More on sensors will be deal with below.

\subsection{C. albicans LYFE-CYCLE AND}

\section{POLYMORPHISM:}

\subsubsection{Morphogenesis and role in virulence:}

An important feature of C. albicans, relevant to its pathogenesis, is its ability to switch between different morphological forms. C. albicans is able to develop single spherical cells including yeast cells and chlamydospores, as well as elongated cells developing into multicellular true hyphae or pseudohyphae. Budding yeast cells can be induced to form true hyphae, which grow by continuous apical extension followed by septation. Pseudohyphae grow, differently from true hyphae, by unipolar budding: buds develop into elongated cells, which remain attached to mother cells, stop growth and then resume budding. Chlamydospores are large, spherical, thick-walled cells, which are usually produced at the ends of filaments on certain nutrient-poor media (Ernst 2000; Staib and Morschhauser 2007).

A crucial component of this versatility is the ability to survive as a commensal in several anatomically distinct sites, each with its own specific set of environmental pressures. Thus, $C$. albicans must be able to adapt its growth to a range of physiological extremes. To achieve adaptability, the fungus has evolved sophisticated mechanisms of sensing and responding to environmental cues by activating developmental switches that result in coordinated changes in cell physiology, morphology, and adherence. Its ability to grow in such a great variety of morphological forms provides an excellent paradigm to understand how signalling pathways coordinate growth and development. Additional interest in the molecular mechanisms of C. albicans 
morphopathogenic determinants

originate from the necessity of identifying new drug targets due to increased drug resistance in clinical isolates (Liu 2001; Biswas, Van Dijck et al. 2007).

The transition between these different morphological forms in response to diverse stimuli seems to be very important for fungal pathogenicity (Lo, Kohler et al. 1997; Chaffin, Lopez-Ribot et al. 1998; Karkowska-Kuleta, RapalaKozik et al. 2009). The traditional criterion for distinguishing between these different forms is cell shape. Hyphae that develop from an unbudded yeast cell have no constriction at the neck of the mother cell and have parallel sides along their entire length. Pseudohyphal cells have a constriction at the neck of the mother cell and the bud and at every subsequent septal junction. Both the width and length of a pseudohyphal cell can vary enormously, so that at one end they resemble hyphae and at the other they resemble yeast cells with elongated buds. However, a characteristic feature of pseudohyphae is that the width of the compartments that make up the filaments is not constant being wider at the centre than at the two ends (Sudbery, Gow et al. 2004)

It is generally accepted now that dimorphism is not only a virulence trait per se, but is also co-regulated with other virulence factors, which are associated with cellular morphology. Dimorphism and other striking morphogenetic phenotypes of $C$. albicans, including chlamidospore formation and phenotypic switching, are regulated by environmental conditions (Ernst 2000).

The ability to switch from the yeast to the hyphal forms is often considered to be necessary for virulence. Both hyphae and pseudohyphae are invasive (i. e. they invade the agar substratum when they grow on laboratory plates). This property could promote tissue penetration during the early stages of infection, whereas the yeast form might be more suited for dissemination in the bloodstream. The filamentous forms might be important for the colonization of organs. The dimorphic transition clearly plays a role in virulence. However, it is important to recognize that most dimorphic fungi that are human pathogens exhibit growth by budding into diseased tissues and exist as filamentous mycelial fungi in the external environment. Therefore, filamentous growth is not obligatory coupled with tissue invasion. Moreover, in C. albicans, mutant strains incapable of hyphal formation are in general avirulent in models of disseminated or mucosal candidiases, but decreased 
infectivity without changes in hyphal formation has been also observed and strains that are unable to grow in the yeast form are also less virulent. Defects other than the inability to form hyphae could be responsible for the reduced virulence of the mutant strains. Invasion should be facilitated by the transition between yeast and hyphae, but other mechanisms have been observed including "persoption" of yeast cells on the gut mucosa into the inner tissues, as well as induced pathogenesis by human esophageal cells. A further complication in the in vivo analysis of pathogenicity comes from the reconignition that the expression of markers used to create mutant strains can significantly influence the measured level of virulence. This problem will require some re-evaluation (Calderone and Fonzi 2001; Romani, Bistoni et al. 2003; Sudbery, Gow et al. 2004; Whiteway and Oberholzer 2004).

\subsubsection{Transcriptional control of morphogenesis:}

Although many different signals can induce filamentous development, the strategies for connecting the external signals to the change in cell differentiation are broadly conserved among fungi. The yeast $S$. cerevisiae is able to grow in filamentous forms but does not have a true hyphal growth phase. Many of the genes that are required for polarity in S. cerevisiae are well conserved in $C$. albicans. The conservation of this machinery suggests that hyphal development does not occur through a totally novel mechanism, but is likely to represent a modulation of an intrinsic mechanism controlling polarized growth (Sanchez-Martinez and Perez-Martin 2001; Whiteway and Oberholzer 2004).

C. albicans morphology is directly related to environmental conditions. Hyphal development depends on two factors: (1) the nature, number and intensity of environmental signals (outside cues) and (2) the activity of signalling pathways including key transcription factors (cellular response machinery) (Ernst 2000).

As shown in Figure 4, environmental cues trigger separate signal-transduction pathways via sensor proteins.

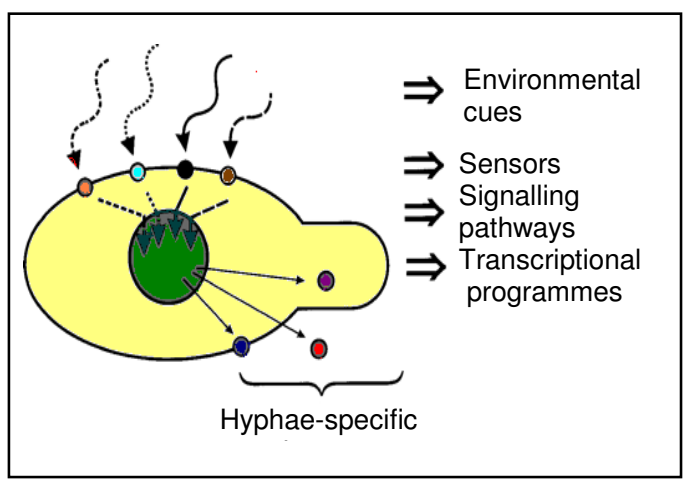

Figure 4. General scheme of hyphae induction. (modified from Ernst 2000 
Yeast to hyphal transition in $C$. albicans is triggered by various environmental cues. Different culture conditions in vitro can reflect the wide range of signals that can promote hyphal growth in many different niches in vivo. Factors that promote hyphal development in vitro are: presence of serum or $\mathrm{N}$-acetylglucosamine (GlcNAc); pH (around 7-7,5); high temperature $\left(37^{\circ} \mathrm{C}\right)$ or presence of $\mathrm{CO} 2$. The most powerful hyphae-inducing protocol in vitro involves incubation of C. albicans at low densities in liquid medium containing serum, GlcNAc or other inducers, at $37^{\circ} \mathrm{C}$. Liquid induction is usually monitored for a few hours, when initial germ tubes have developed into hyphae. In contrast, on solid media cells develop into colonies over several days of growth, until (pseudo-) hyphal growth emerging from the colonies is recorded (Ernst 2000; Liu 2001; Whiteway and Oberholzer 2004)

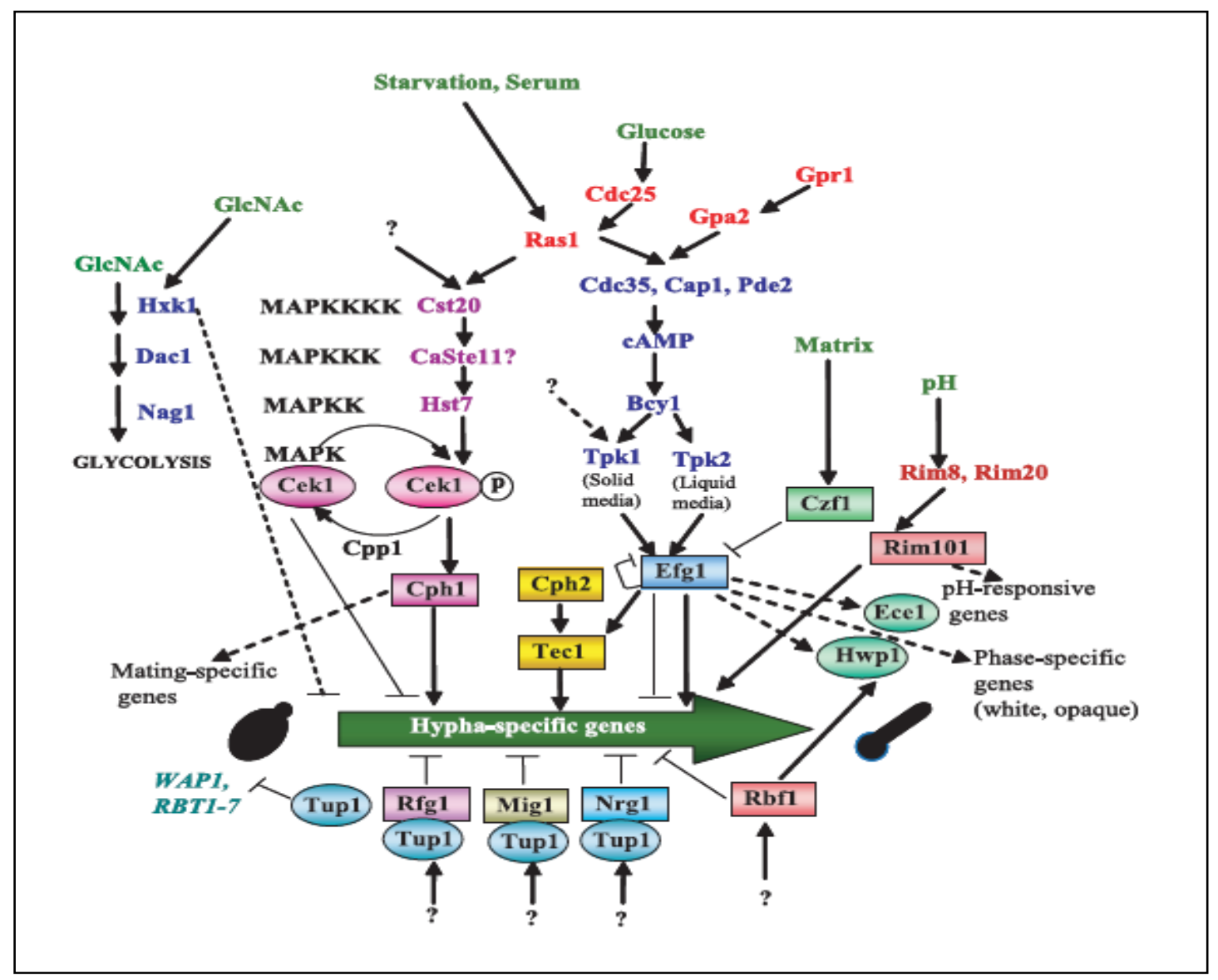

Figure 5. Regulation of dimorphism in C. albicans by multiple signaling pathways. Transcription factors are shown in rectangular boxes (from Biswas, Van Dijck et al. 2007). 
In recent years, receptors/sensors that may mediate environmental responses have been identified and partially characterized. Different works have aimed in identifying receptors, ligands and signaling pathways involved in the sensing of environmental signals in $C$. albicans. Signaling pathways that regulate dimorphism are summarized in Figure 5. The Cph1-mediated MAPK pathway and the Efg1-mediated cAMP pathway are well characterized signaling pathways in dimorphic transition regulation. In C. albicans, Ras1p is an important regulator of hyphal development and likely functions upstream of both pathways. In the cAMP-PKA pathway, two catalytic subunits or isoforms of PKA, Tpk1p and Tpk2p, have differential effects on hyphal morphogenesis under different hyphal-inducing conditions. The MAPK cascade includes Cst20p (PAK), Hst7p (MAPKK), Cek1p (MAPK), and the downstream transcription factor Cph1p, which is a homolog of the $S$. cerevisiae transcription factor Ste12. Positive regulators of hyphal formation are Efg1p, Cph1p, Cph2p, Tec1p, Rim101p and Czf1. Transcription of the hyphal regulator TECl is regulated by Efg1p and Cph2p. Rim 101p or Czf1p may function through Efg1p or act in parallel with Efg1p. Tup1p is the negative regulator of the hyphal transition. Tup1p, recruited by $\operatorname{Rfg} 1 \mathrm{p}, \mathrm{Nrg} 1 \mathrm{p}$, or Mig1p, and Rbf1p are also implicated in dimorphic transition. GlcNAc-inducible hexokinase, Hxk1p, plays a negative role in hyphal development under certain conditions (Biswas, Van Dijck et al. 2007).

These signal transduction pathways form a complex and interconnected network that integrates environmental cues to control morphogenesis and transcription appropriately. The existence of integration within this network is well illustrated by the transcription factor Efg1p, which receives information through multiple pathways and is involved in different programs such as hyphal development, chlamidospore formation, white-opaque switching and assembly of biofilms. The specificity of the response may be achieved by phase-specific activation of EFG1 transcription, by modulation of Efg1p levels, by post-translational modifications of Efg1p, or by other transcription factors of the network that function synergistically with or antagonistically to Efg1p (Whiteway and Oberholzer 2004).

Distinct filamentation signaling pathways converge to regulate a common set of differentially expressed genes. Importantly, most of the genes 
regulated by multiple filamentation pathways encode known virulence factors. Perhaps, C. albicans utilizes converging pathways to regulate its vital virulence factors to ensure its survival and pathogenicity in various host environments (Lane, Birse et al. 2001).

The hypha-specific genes (HSGs) identified so far include ECE1, $H W P 1$, HYR1, ALS3, ALS8, RBT1 and RBT4. They encode either cell wall or secreted proteins and many have been shown to be important virulence factors for systemic infection. HWPI encodes a cell-wall protein that can serve as a target for mammalian transglutaminases to form covalent attachments between $C$. albicans and host epithelial cells. RBT1 encodes a cell-wall protein, and RBT4 encodes a secreted protein similar to a set o pathogeneis-related proteins from plants. Both genes are necessary for the virulence of $C$. albicans in systemic mouse model. In addition, three members of the secreted aspartyl proteinase genes, SAPS 4-6, are differentially transcribed when hyphal development is induced with serum or media containing polypetides as the sole nitrogen source. They have been shown to promote virulence in host systemic and mucosal candidal infections. The expression of HSGs seems to be regulated by multiple pathways.
Cooperative interaction between hyphal regulators of different pathways at the promoters of hyphae-specific genes and pathways crosstalk are other potential mechanisms for the observed convergence in the regulation of HSGs expression (Lane, Birse et al. 2001; Liu 2001). In addition to hyphae specific genes, many dimorphic regulators or signalling pathways have also pathwayspecific genes. For example, PHRI and PHR2 expression is regulated by Rim101p. The expression of pathwayspecific genes and hypha-specific genes are not exclusive to each other. It is likely that, under any given conditions, a network of signalling pathways are employed to simultaneously assess the availability of multiple nutrients, cell density and other growth conditions. The integrated output of these pathways determines the gene expression and dimorphic transition (Liu 2001).

\subsubsection{Morphogenesis checkpoint and cell cycle progression:}

Despite the apparent importance of morphological switching to the virulence of $C$. albicans, little is known about the fundamental biological differences which coordinate cell cycle progression 
and morphology and how these

differences regulate morphological switching (Finley and Berman 2005).

Although connections between morphology switches, cell cycle checkpoints and cell cycle regulators have been actively studied in $C$. albicans, the absence of the cell cycle dependent transcriptional expression pattern has limited these analyses, because synchronization methods have not provided a complete picture of the $C$. albicans cell cycle (Cote, Hogues et al. 2009). As shown in Figure 6, the organization of the first pseudohyphal cell cycle is not substantially different from that of a yeast cell, except for bud elongation and failure to separate after septum formation. Therefore, similarly to what occurs in a yeast cell cycle, in the first pseudohpyphal cell cycle, a septin ring appears at the neck between the mother cell and the daughter cell before bud emergence. Mitosis takes place across the plane of the septin ring. When mitosis is complete, the septin ring separates into two rings and a primary septum composed of chitin is formed between them. As in budding yeast, septum formation involves the appearance and contraction of an actomyosin ring (Sudbery, Gow et al. 2004).

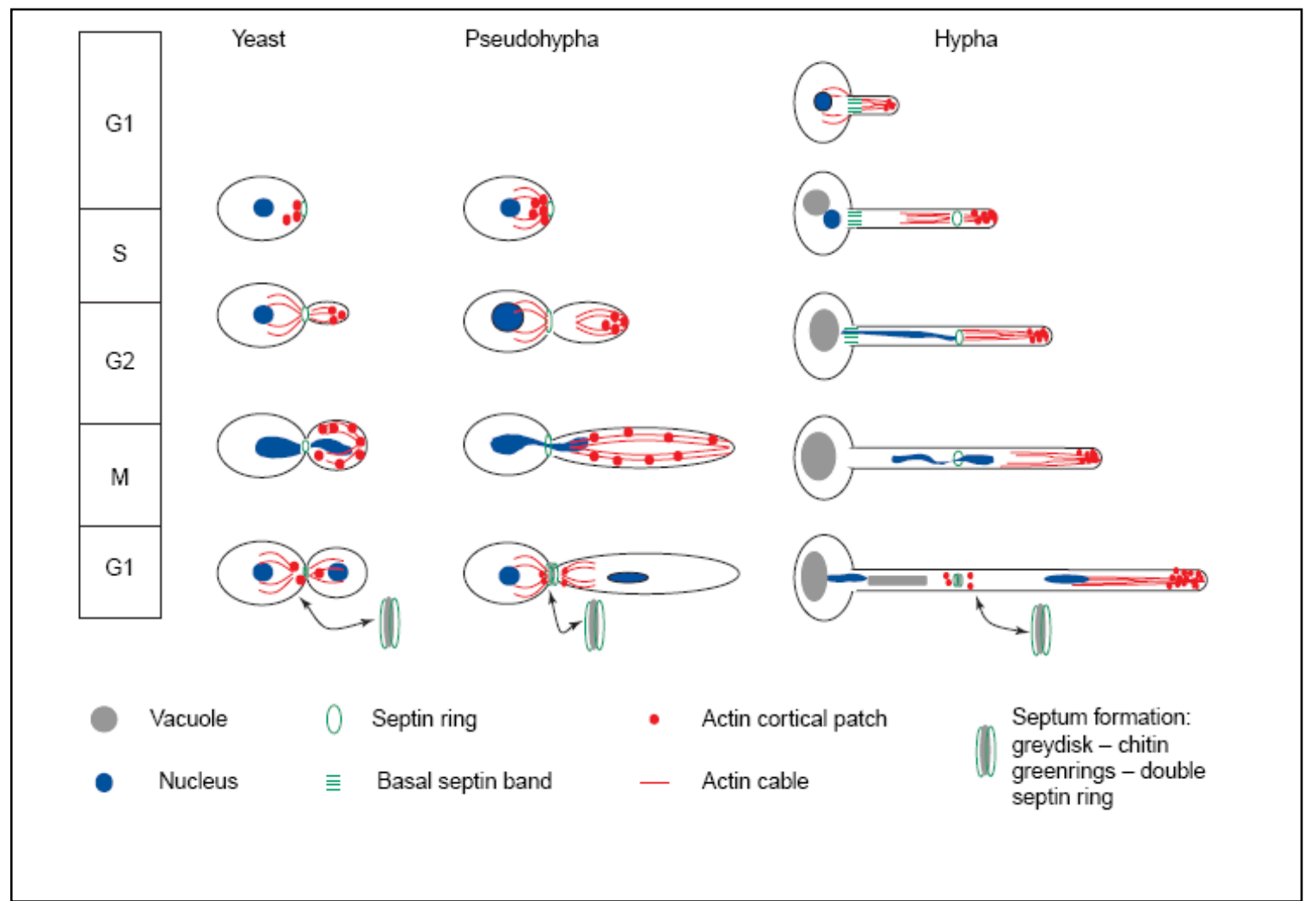

Figure 6. Representation of the cell cycle of yeast and the first cycle of hyphae and pseudohyphae induced from unbudded yeast cells (from Sudbery, Gow et al. 2004). 
By contrast, the first hyphal cell cycle shows substantial differences from yeast and pseudohypha which are summarized in the following: (i) In the yeast form, budding occurs either next to the bud scar from the previous cell cycle (axial pattern), or at the opposite end of the cell from which the previous bud formed (bipolar pattern) in a manner that is temperature-dependent. When germ tubes evaginate from yeast cells, 50\% appear at random sites on the mother cell whereas the remainder evaginate with a bipolar pattern relative to the previous bud site. (ii) Germ tube initiation occurs before the start of the cell cycle which is defined by two landmark events,spindle pole body duplication and DNA synthesis. On opposite, in yeast cells, bud emerging depends on the beginning of the cell cycle and occurs shortly after the two landmark events. (iii) The first septum forms at the mother-bud neck in yeast and pseudohyphae but within the germ tube in hyphae. (iv) Germ tubes have a basal band of longitudinal septin bars around the germ tube neck. This structure is distinct from the true septin ring that appears along the germ tube length and organizes the formation of the first septum. The septin ring is equivalent to that forming at the bud neck in yeast and pseudohyphae at the time of bud formation. It has probably the same structure as similar filament rings are observed by electron microscopy at the bud neck in yeast and within the germ tube in hyphae (Hazan, Sepulveda-Becerra et al. 2002) (v) The first nuclear division occurs across the mother bud neck in pseudohyphae and yeast, whereas in hyphae the nucleus migrates out of the mother cell and divides within the germ tube. Although mitosis takes place across the plane of the septin rings, these rings are not necessary for nuclear migration or mitosis, because mitosis takes place normally in a mutant deleted in GIN4, a gene that is required for the organization of the septin ring (Sudbery, Gow et al. 2004).

During the first cell cycle in germ tubes, a large vacuole appears in the mother cell. During the process of septum formation, most of the cytoplasm is transferred to the apical compartment, which continues to grow and progress through the cell cycle. After completion of the first cell cycle, the highly vacuolated mother cell remains in the $G_{1}$ phase. This pattern is repeated so that subapical compartments are also vacuolated and remain in $G_{1}$. These subapical compartments slowly accumulate cytoplasmic mass at the expense of vacuoles until they have reached a critical size threshold that 
allows them to re-enter the cell cycle by forming either a branch near an existing septum or a second germ tube from the mother cell (Hazan, Sepulveda-Becerra et al. 2002; Sudbery, Gow et al. 2004).

\section{Role of actin cytoskeleton:}

During hyphal development, cell surface expansion is highly restricted to the apical tip of hyphae. This polarized apical growth requires the actin cytoskeleton. C. albicans yeast cells display a temporal change in the organization of the actin cytoskeleton during cell cycle progression, like that in $S$. cerevisiae cells, whereas the actin cytoskeleton is polarized at the tip of all hyphal cells during filamentation (Figure 6). In S. cerevisiae and other organisms, the small GTPase of the Rho subfamily Cdc42 is known to be critical for establishing a polarized actin cytoskeleton in response to extracellular stimuli. C. albicans $\mathrm{Cdc} 42$ is also essential for cell morphogenesis and is known to be a master regulator of actin organization, essential for polarity establishment (Liu 2001; Fischer, Zekert et al. 2008).

\section{Role of microtubules:}

One clear difference between the various $C$. albicans morphological states is the spatial coordination of nuclear dynamics and morphology. Nuclear dynamics is most dramatic in hypha: nuclei migrate into germ tubes and divide across the future site of septation 10 to $20 \mu \mathrm{m}$ away from the basal mother cell. Mother nuclei then return to the basal cell while daughter nuclei migrate toward the growing tip. In yeast and pseudohyphae, mitosis resembles that of the budding yeast Saccharomyces cerevisiae where nuclei divide across the bud neck. Thus, mitosis occurs across the site of septation irrespective of cell morphology. This suggests that nuclear position varies as a function of morphology (Finley and Berman 2005). How nuclei migrate to the site of septation in C.albicans hyphae is unclear. However, it is likely to involve the microtubule (MT) cytoskeleton, the organization of which is similar to that of $S$. cerevisiae. MTs in C. albicans are involved in positioning nuclei and regulating hyphae morphogenesis. Figure 7 shows a model of nuclear and microtubule dynamics during hyphal cell cycle in $C$. albicans. During the $\mathrm{G}_{0}$ to $\mathrm{G}_{1}$ transition, tubulin saturates the cytosol of unbudded cells and short hyphae. Once a hypha reaches a critical size, it enters into $\mathrm{S}$ phase: the septin ring appears, SPBs duplicate, and the MT sliding machinery is activated. As the hypha proceeds into $G_{2}$, tubulin is 
incorporated into the developing spindle and the number of free MTs is reduced. Also in $\mathrm{G}_{2}$, repetitive sliding events from SPB-bound MTs facilitate nuclear migration to the septin ring. In early $\mathrm{M}$ phase, the short spindle approaches the ring and the hypha undergoes the transition into anaphase. This transition is also dependent upon the attainment of a critical size by hypha. During anaphase A, kinetochore MTs shorten and pull the chromosomes to a short distance from the spindle poles. Subsequently, during anaphase B, interpolar MTs push the spindle poles apart, preferentially pushing the mother nucleus toward the hyphal neck. As the spindle disassembles, the concentration of cytosolic tubulin reaches saturation and free MTs reappear. Following septation, a slower, unknown mechanism moves the $\mathrm{G}_{0}$ mother nucleus back into the basal cell. Thus, in C. albicans hyphae, nuclei and MTs exhibit behaviors that, in some ways, resemble those of $S$. cerevisiae (appearance of the septin ring prior to nuclear movement and nuclear movement to and division across the septin ring) and in other ways are more like those of other organisms (cell cycleregulated MT cytoskeleton) (Finley and Berman 2005).

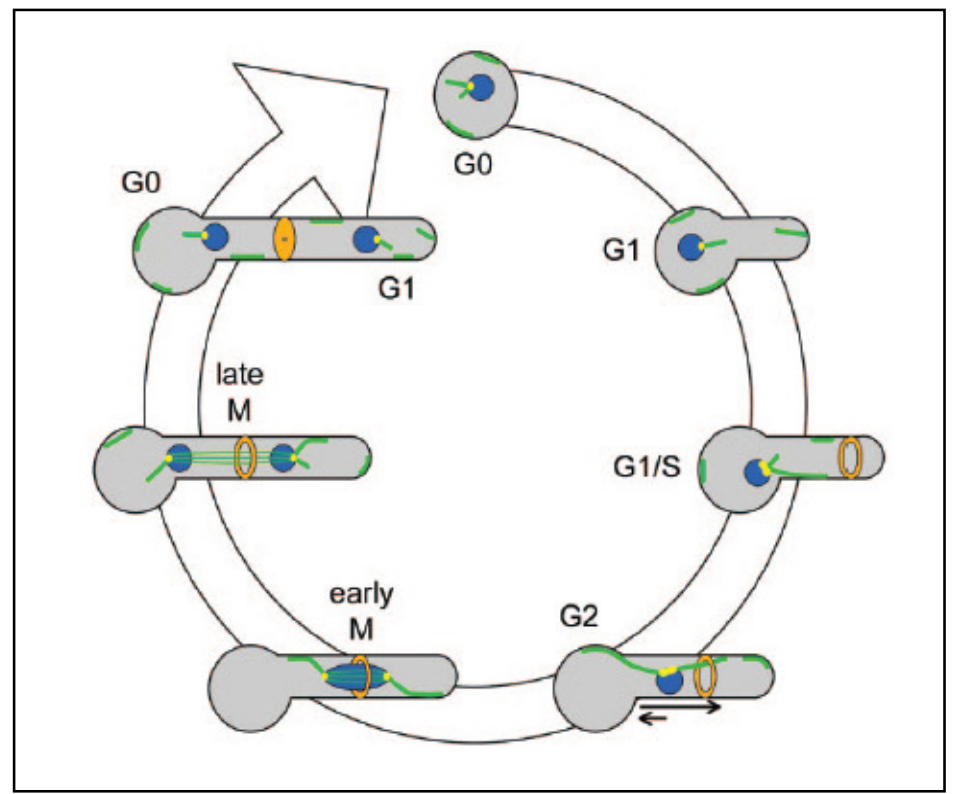

Figure 7. Model of nuclear and microtubule dynamics during a hyphal cell cycle in C.albicans. Nuclei are in dark blue, SPBs yellow, MTs in green, preseptum is represented as a narrow orange ring and septum as a thick orange ring (from Finley and Berman 2005). 
1.5. HOST-PATHOGEN

\section{INTERACTIONS :}

An understanding of the hostpathogen interactions that allow Candida albicans to switch from a commensal to a pathogen capable of infecting a variety of tissues would benefit the design of innovative approaches of the prophylaxis and therapy of this widespread and common infection. Binding of the organism to host cells, host cell proteins or microbial competitors (coaggregation) more than likely prevents or at least reduces the extent of clearance by the host (Calderone and Fonzi 2001; Rupp 2007; Richardson and Rautemaa 2009). One or the first steps of infection of the host is the attachment of the pathogen to the surface of host tissues. This step of the host-pathogen interaction is crucial for colonization by the pathogen and for the persistance of the pathogen in the host (Rupp 2007). Adhesion and invasion of Candida albicans to host tissues will be analyzed in some regards in this thesis.

\subsubsection{Adhesion to host tissues:}

Candida albicans expresses several adhesin proteins, including Als proteins, Hwp1p, Eap1p, Csh1p, and other cell surface proteins that promote attachment the host. $A L S$ gene family encode for proteins that mediate adhesion to epithelia, yeast aggregation and biofilm formation. The family is encoded at eight loci. The various forms are expressed at different phases of growth and infection. Reverse transcription PCR tests have detected $A L S$ gene expression in human clinical specimens and in a vaginal candidiasis model. Although transcription from all $A L S$ genes has been observed, ALS1, ALS3, and ALS9 were detected most frequently. Similar results for $A L S$ transcriptional activity have been found in a murine model of disseminated candidiasis. Experiments utilizing the gene encoding the yeast enhanced green fluorescent protein (yEGFP) as a reporter gene under control of $A L S$ promoters suggests that some Als proteins (mainly Als1p and Als3p) are abundant on the $C$. albicans cell surface, while others are produced at lower levels in the mouse disseminated candidiasis model. Als adhesins mediate both adhesion to mammalian tissues and aggregation of the $C$. albicans cells to form microscopic colonies (Dranginis, Rauceo et al. 2007; Karkowska-Kuleta, Rapala-Kozik et al. 2009).

C. albicans Hwp1p, a glutamine-rich, GPI wall-anchored adhesin, is a substrate for epithelial cell transglutaminases. Thus, it participates in a covalent cross-link between the 
yeast and the epithelium. The resulting association would be shear-resistant, extremely close, and permanent in the absence of proteolysis. Such interactions could underlie the clinical observation that oropharyngeal colonies of $C$. albicans resist removal by scraping (Naglik, Fostira et al. 2006)

Other $C$. albicans adhesins are Eap1p and Csh1p. Eap1p of C. albicans mediates binding to plastic surfaces in a manner resistant to shear forces. Theses properties are those required for stable adhesion to indwelling catheters and other devices, so the protein may be critical in prosthesis-induced candidemias and endocarditis. Eap1p has the same general architecture as the GPIcross-linked lectins and peptide-binding proteins. Chs1p of $C$. albicans is known to enhance hydrophobicity of $C$. albicans cells, also facilitating receptorligand interactions (Dranginis, Rauceo et al. 2007; Karkowska-Kuleta, RapalaKozik et al. 2009).

\subsubsection{Invasion process in Candida albicans:}

The first step for most microbial pathogens to cause an infection, but also of microbes to establish a commensal relationship, is the attachement to epithelial surfaces. Thus, pathogens must adhere to a host cell in order to colonize any given host tissue and as a prerequisite for survival and distribution. Pathogenic fungi interact with a variety of host cells during the induction of disease. To cross tissue planes and cause invasive disease, these organisms must invade normally nonphagocytic host cells such as epithelial cells and endothelial cells (Filler and Sheppard 2006; Thewes, Kretschmar et al. 2007; Zakikhany, Thewes et al. 2008; Karkowska-Kuleta, Rapala-Kozik et al. 2009).

In $C$. albicans many different factors are known to be responsible for the fungus to become a pathogen. Intuitively, invasion should be facilitated by the transition between yeast and filamentous growth. In fact, the latter statement is partially true, but other mechanisms such as persoption of yeast cells on the gut mucosa into the inner tissues have been observed as well as induced phagocytosis by human esophagel cells (Calderone and Fonzi 2001). Moreover, other factors should be considered, such as adhesion and adhesion molecules which are required at the first step in the host-pathogen interaction or hydrolytic enzymes that contribute to host tissue invasion by digesting or destroying cell membranes and by degrading host surface molecules (Staib, Kretschmar et al. 2000; 
Calderone and Fonzi 2001; Schaller, Borelli et al. 2005).

In a recent study (Zakikhany, Thewes et al. 2008), two types of reconstituted human tissues (RHE), an oral epithelial tissue and a reconstituted liver tissue, have been used to study the invasion of host cells by $C$. albicans. Dissection of the different stages of fungus-host interactions from attachment via invasion to tissue destruction was performed by using the oral epithelial model. Electron microscopy pictures revealed that contact of hyphae, but not of yeast cells, to epithelial cells in the early phase frequently caused host response mechanisms such as the formation of epithelial cell protrusions surrounding the hyphae and membrane ruffling characteristic of induced endocytosis.

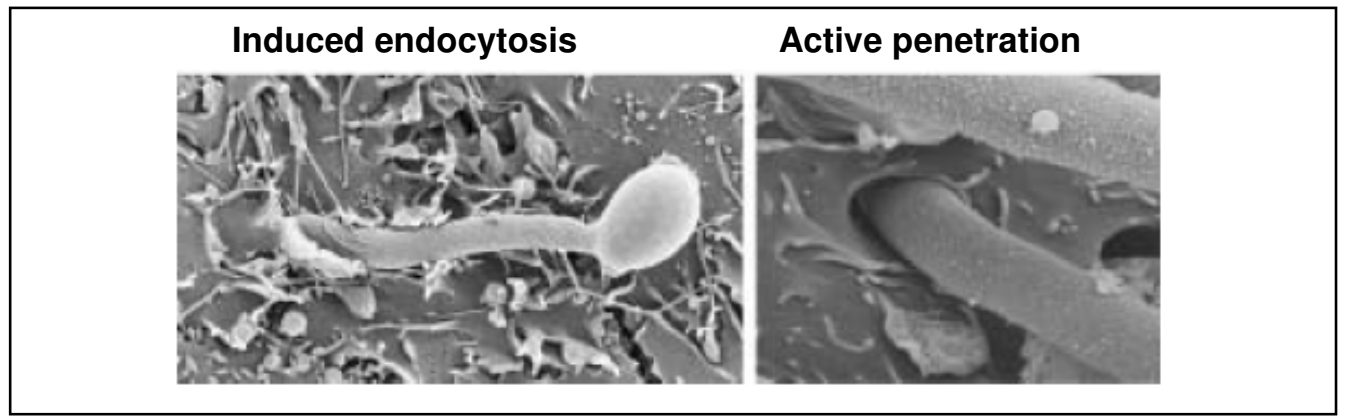

Figure 8. Entry of $C$. albicans into human host cells. Images from an electron microscope of a model of reconstituted human oral epithelium infected with $C$. albicans. Two disctinct mechanisms are shown: a passive one, in which $C$. albicans hypha are taken up by the host cells via induced endocytosis (associated with membrane ruffluing) and an active one, in which $C$. albicans hyphae penetrates into the host cell. (from Zakikhany, Thewes et al. 2008)

This induced endocytosis had been previously described to be mediated by the GPI-anchored adhesion protein Als3 (Phan, Myers et al. 2007). Despite these interactions, no tissue damage in the early phase (1-6 h) was observed. However, tissue damage increased dramatically after $12 \mathrm{~h}$, when hyphae penetrated the tissue not only in the top layer, but also in deeper epithelial cell layers. In this phase, penetration due to physical forces seemed to be the dominant mode of invasion. In summary, experimental oral infection based on RHE can be divided into three phases: attachment, invasion and tissue destruction (Zakikhany, Thewes et al. 2008). 


\section{THE FUNGAL CELL WALL}

\subsection{GENERAL OVERVIEW:}

Yeast and fungi are surrounded by a cell wall that is a complex structure essential for maintenance of the cell shape, prevention of lysis, and protection against harmful environmental conditions. By its definition, the cell wall appears to be static. However, the cell wall is constantly changing its size and shape, to accompany the growth of the cell. Indeed, it is the cell wall that confers a particular shape to the fungal cells. In mycelial fungi, the advance of the organism in search for nutrients is led by the cell wall and so in infections caused by pathogenic fungi. During cytokinesis, a specialized variant of the cell wall, the septum, is formed to separate the two dividing cells. Thus, synthesis of the cell wall and septum can and has been used as a model for morphogenesis. Because the formation of these structures must be co-ordinated with growth and cell division, study of the mechanisms that regulate their synthesis can also provide new information about cell cycle controls. Finally, inhibition of cell wall synthesis and septum formation is incompatible with cell growth, therefore these processes are promising targets for new antifungal agents (Cabib, Drgon et al. 1997; Lagorce, Hauser et al. 2003).

\subsection{STRUCTURE}

AND

COMPOSITION OF THE FUNGAL

\section{CEL WALL:}

Cell structure seldom stops at the plasma membrane boundary but usually extend beyond it in an extracellular network of polysaccharides and proteins. Among the eukaryotes, extracellular matrix composition is sufficiently different between the metazoan, fungal and plant kingdoms that a research area has been independently developed for each, with little common ground. Fungi and plants have extracellular matrices containing polysaccharide-protein complexes termed cell walls. Cell walls allow fungi and plants to build structures based on the use of cells as hydrostatic bricks, a process that is particularly well elaborated in woody plants. Despite a common body plan, the actual polymers used by fungi and plants to construct cell walls are often different (Lesage and Bussey 2006).

S. cerevisiae consumes a large amount of metabolic energy to build the cell wall that represents about $20 \%$ of its dry weight. The yeast cell wall consists mainly of polysaccharides made up of three sugars, glucose, mannose, and $\mathrm{N}$ - 
acetylglucosamine (GlcNAc).Glucose

residues are linked to other glucose

molecules through $\beta-(1,3)$ and $\beta-(1,6)$

linkages and to GlcNAc via $\beta-(1,4)$

bonds. Mannoproteins can be linked to $\beta$-(1,6)-glucose chains through a processed glycosylphosphatidylinositol (GPI) anchor or to $\beta$-(1,3)-glucan through an alkali-labile bond. The structure of the cell wall is illustrated in Figure 9. Chains of $\beta$-(1,3)-linked glucose residues are branched by $\beta-(1,6)$ linkages, forming a fibrillar $\beta-(1,3)$ - glucan which serves as a backbone to which chitin, $\beta-(1,6)$-glucan, and some mannoproteins are linked. In addition, the $\quad \beta$-(1,6)-glucan and GPImannoproteins are linked together via a remnant of their GPI anchors. The vegetative cell wall has a layered ultrastructure as observed by electron microscopy, with an inner layer of glucans and chitin and an outer layer of mannoproteins (de Groot, de Boer et al. 2004; Lesage and Bussey 2006).

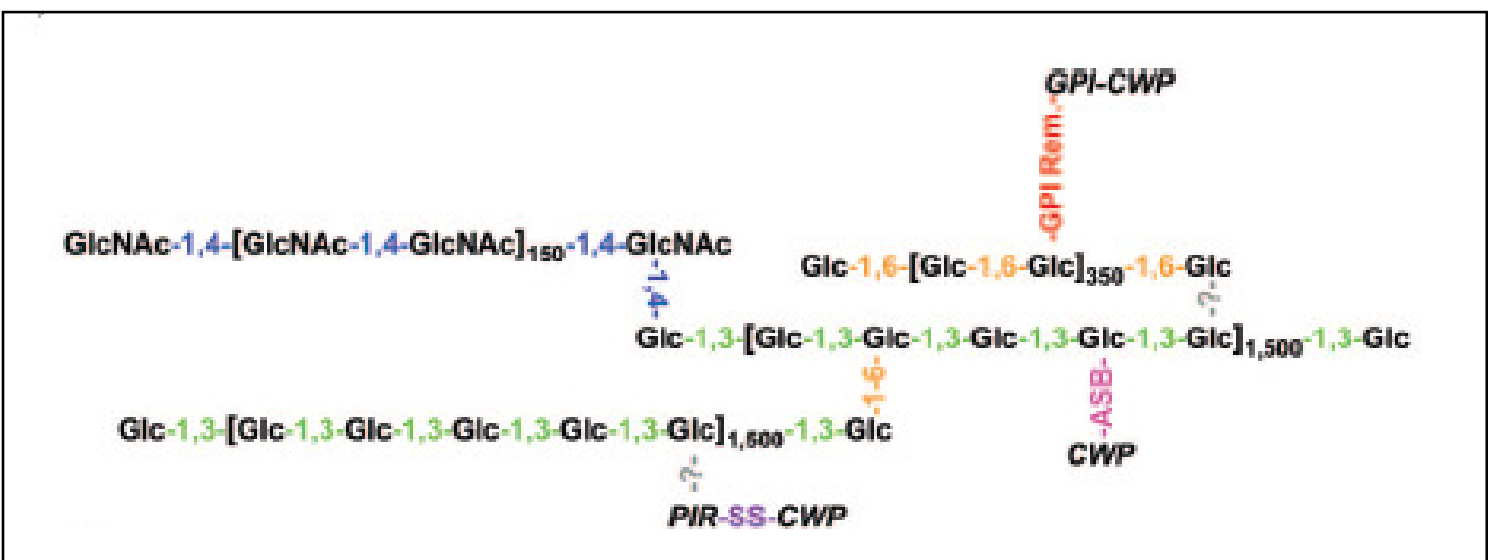

Figure 9. Schematic representation of the cell wall components and their linkages (from Lesange and Bussey 2006). Cell wall mannoproteins (CWP) can be linked to the $\beta$-(1,3)-glucan via alkali-sensitive Bonds (ASB) or to PIR proteins (PIR) via a sidulfide link (SS). GPI cell wall proteins are attached to the $\beta$-1,6-glucan through a remmant GPI anchor (GPI-Rem).

\subsection{1. $\beta-(1,3)-$ glucan network:}

The $\beta$-glucans are glucose polymers that account for half of the cell wall dry weight. In $S$. cerevisiae, approximately $80 \%$ of these $\beta$-glucans are $\beta$-(1,3)-linked. The $\beta$-(1,3)-glucan chains, with a degree of polymerization of $\sim 1500$ glucose units/chain, have a coiled spring-like structure that confers elasticity and tensile strength to the cell wall (Kollar, Petrakova et al. 1995; Dijkgraaf, Abe et al. 2002; Lesage and Bussey 2006).

This polymer is synthesized at the cell surface by a specific biosynthetic enzyme, the $\beta$-(1,3)-glucan synthase (GS), which 
uses cytosolic UDP-glucose as a substrate.

The $\beta$-(1,3)-glucan synthase complex, located in the plasma membrane extrudes the polymer outside of the cell and other families of enzymes are responsible for its incorporation in the cell wall (Abe, Nishida et al. 2001; Dijkgraaf, Abe et al. 2002). $\beta-(1,3)$-glucan synthase (GS) is composed of at least two subunits. In $S$. cerevisiae, the putative catalytic subunit is encoded by two related genes, FKS1 and $F K S 2$, and is predicted to be an intrinsic membrane protein with 16transmembrane domains. The regulatory subunit is a peripheral membrane protein encode by RHOl (Abe, Nishida et al. 2001). Rho1p is associated to the plasma membrane through a prenyl tail that is essential for its localization and function (Inoue, Takewaki et al. 1995). Like other small G proteins, Rho1 cycles between an active GTP-bound state and an inactive GDP-bound state under modulation of GDP-GTP exchange factors (GEFs) and by GTPase activating proteins (GAPs). Rom $2 p$ and $\operatorname{Lrg} 1 p$ are the respective GEF and GAP specialized for the regulation of $\beta$-(1,3)-glucan synthase (Roumanie, Weinachter et al. 2001; Levin 2005).

In C.albicans a gene that encodes a target for $\beta$-(1,3)-D-glucan synthase inhibitors, named CaFKSI was identified in 1997 (Douglas, D'Ippolito et al. 1997). CaFKS1, has significant homology to the
Saccharomyces cerevisiae FKS1 and FKS2 genes, which encode partially functionally redundant subunits of GS. An homologous of the Rho1 GTPase has also been identified in $C$. albicans and it has been shown to act in the same manner as Saccharomyces cerevisiae Rholp to regulate $\beta$-(1,3)-glucan synthesis.

The development of inhibitors of the synthesis of fungal cell wall glucan represents an important advancement in antifungal chemotherapy. Known $\beta$-(1,3)D-glucan synthase inhibitors include the acidic terpenoids, the papulocandins, and the echinocandins, cyclic hexapeptides that are N-linked to a fatty acyl side chain (Onishi, Meinz et al. 2000; Douglas 2001). Caspofungin (L-743,872, MK0991), an echinocandin, was the first of these noncompetitive inhibitors to become available in the United States, where it is marketed as Cancidas $₫($ Merck), and in Europe, where it is marketed as Caspofungin MSD ® (Merck). Caspofungin received in 2003 the approval of the US Food and Drug Administration (FDA) for the treatment of oropharyngeal and esophageal candidiasis and invasive candidiasis in adults. Despite the clinical efficacy of Caspofungin as an antifungal agent it has been shown that in laboratory mutants as well as in some clinical isolates, mutations in the FKS1 gene result in amino acid changes in the 
protein which are sufficient to confer a reduced susceptibility to Caspofungin (Desnos-Ollivier, Bretagne et al. 2008). Moreover evidences obtained by treating Candida albicans cells with Echinocandin indicate that resistant cells may arise also because of the activation of a compensatory response which involves hyperaccumulation of chitin (Walker, Gow et al. 2009).

\subsection{2. $\beta$-(1,6)-glucan:}

The remaining $20 \%$ of the $\beta$-glucan in $S$. cerevisiae is $\beta$-1,6-linked. $\beta-(1,6)-$ glucan polymer has an average chain length of $\sim 350$ glucose residues, with the $\beta-(1,6)$ backbone branched with $\beta-(1,6)$ side chains via 3,6-substituted glucose residues on $\sim 15 \%$ of the residues. This polymer has a glue-like function in the cell wall, interconnecting all major components (Kollar, Reinhold et al. 1997; Lesage and Bussey 2006).

Many genes involved in $\beta$-(1,6)-glucan biosynthesis have been identified in $S$. cerevisiae through mutations that confer resistance to the $\mathrm{K} 1$ killer toxin, a poreforming protein that binds to a $\beta-(1,6)$ glucan-containing cell surface receptor, however their role in the synthesis of the polymer is still unclear. The KRE (killer toxin resistant) gene products have been localized along the secretory pathway and at the cell surface (Shahinian and Bussey 2000; Dijkgraaf, Abe et al. 2002). Out of these genes, KRE5 encodes a protein that harbours the endoplasmic reticulum retention signal (Meaden, Hill et al. 1990), KRE6 and the multicopy suppressor of the kre6 $\Delta$ null phenotype, $S K N 1$, code for homologous proteins that contain the putative UDP-glucose-binding consensus sequence (Roemer and Bussey 1991; Roemer, Delaney et al. 1993) and localize in the Golgi subcompartments, whereas Kre2p functions as $\alpha-1,2$ mannosyltransferase for protein $\mathrm{O}$ glycosylation (Hausler, Ballou et al. 1992; Lussier, Sdicu et al. 1995). It has also been demonstrated that Kre1p and Kre9p are O-glycosylated cell surface proteins and contribute to the production of $\beta$ $(1,6)$-glucan polymers (Brown and Bussey 1993; Roemer and Bussey 1995). One of the hypothesis that arises from these observations is that the synthesis of $\beta$ $(1,6)$-D-glucan begins in the endoplasmic reticulum, the product is extended in the Golgi and the final processing steps take place at the cell surface. In Schizosaccharomyces pombe, the polymer has been detected in the Golgi; however, attempts to detect intracellular $\beta$-(1, 6)-Dglucan in $S$. cerevisiae have been unsuccessful leaving open the possibility that synthesis occurs at the plasma 
membrane. A clear molecular description

of the synthesis of $\beta$-(1,6)-glucan is still lacking.

In $C$. albicans cell walls, $\beta$-(1,6)-glucan is particularly abundant, being present at almost double the amount found in Saccharomyces cerevisiae (Mio, YamadaOkabe et al. 1997). In C. albicans homologous of KRE genes of $S$. cerevisiae have been identified supporting the idea that the mechanism by which the $K R E$ genes are involved in $\beta$-(1,6)-glucan synthesis is generally conserved in yeast cell wall biosynthesis. The KRE homologs identified so far in C. albicans are: KRE1, KRE6, SKN1, KRE9 and KRE5. The first $K R E$ homolog identified in C. albicans was KRE1, which was isolated by Boone et al. by its abilitly to complement a krels mutation in $S$. cerevisiae and confer sensitivity to killer toxin (Boone, Sdicu et al. 1991). Later on, cDNA for $C$. albicans homologs of S. cervisiae KRE6 and SKN1 genes were also isolated. The hemizygous kre6 $6 \Delta$ mutation resulted in a reduction of more than $80 \%$ of the cell wall $\beta-(1,6)$-glucan but no obvious change in the amount of cell wall $\beta$ glucan in the homozygous Candida albicans skn1s null mutants. Therefore, only C. albicans KRE6 is active and SKN1 is redundant in terms of $\beta$-(1,6)-glucan synthesis (Mio, Yamada-Okabe et al.
1997). The functional homolog of the $S$. cerevisiae KRE9 has also been isolated in C. albicans. CaKRE9 gene is essential and thus, the CaKRE9 gene product is a potentially useful candidate as a target for fungal-specific drugs (Lussier, Sdicu et al. 1998). Another KRE homolog in $C$. albicans is KRE5. Candida albicans Kre5p is involved in morphogenesis, cell wall construction, dimorphic transition and adhesion to epithelial cells and it is essential for the virulence of $C$. albicans. Thus, Kre5p is also a potentially good target for the development of antifungal drugs (Mio, Yamada-Okabe et al. 1997; Herrero, Magnelli et al. 2004).

Recently the discovery of a small molecule inhibitor of $\beta$-(1,6)-glucan biosynthesis in yeast has been reported. Interestingly, studies of one of its derivates, D21-6076, revealed that although it only weakly reduced the growth of Candida albicans in conventional media, it significantly prolonged the survival of mice infected by the pathogen. Biochemical evaluation of D21-6076 indicated that it inhibited $\beta$ $(1,6)$-glucan synthesis of $C$. albicans, causing a release into the medium of the cell wall proteins, which are known to play a critical role in its virulence. Interestingly, adhesion of C. albicans cells to mammalian cells and elongation of hyphae were strongly reduced by the 
drug treatment. Moreover, evidences suggest that it probably exhibited in vivo efficacy against $C$. albicans by inhibiting its invasion process (Kitamura, Someya et al. 2009). This strongly supports the notion that $\beta$-(1,6)-glucan synthesis may be a promising target for antifungal drugs.

\subsubsection{Chitin:}

Chitin is a linear polymer of $\beta-(1,4)$ linked GlcNAc that forms microfibrils stabilized by hydrogen bonds. It is a minor constituent of the $S$. cerevisiae lateral wall ( 1 to $2 \%$ cell wall dry weight) but it has a crucial role in morphogenesis and is required for cell viability (Cid, Duran et al. 1995; Cabib, Roh et al. 2001). Chitin is found in three locations: at the primary septum, largely in free form, at the mother-bud neck, partially linked to $\beta$ (1,3)-glucan and in the lateral wall, attached in part to $\beta$-(1,3)-glucan. Chitin deposition within the cell is precisely controlled both spatially and temporally. At late $G_{1}$, it is deposited as a ring at the site of bud emergence, then as a disk (the primary septum), and finally in the lateral cell wall of the mother cell after septation (Bulawa 1993; Lesage and Bussey 2006; Cabib, Blanco et al. 2007).

The enzymatic synthesis of chitin in yeast has been extensively studied. Three chitin synthases encoding genes, CHSI,
CHSII, and CHSIII, have been identified and characterized in S. cerevisiae and the genes believe to code for their catalytic components, CHS1, CHSII and CHSIII have been cloned (Orlean 1987; Silverman, Sburlati et al. 1988; Cabib, Sburlati et al. 1989; Choi, Sburlati et al. 1994). All three chitin synthases are membrane-bound proteins, localized in the plasma membrane and in intracellular vesicles compartments of uncertain origin that have been named chitosomes (Ford, Shaw et al. 1996). In S. cerevisiae, Chs3p is responsible for the majority of the chitin deposited in its vegetative cell wall, Chs $2 p$ is specifically required for primary septum formation (Shaw, Mol et al. 1991) whereas Chs1p has a repair function (Cabib, Sburlati et al. 1989; Cabib, Roh et al. 2001). Several proteins are required for Chs3p activity. It has been demonstrated that different proteins participate in the intracellular sorting of Chs3p to the plasma membrane. Exit of Chs3p from the ER depends on Chs7p (Trilla, Cos et al. 1997). Chs5p plays a role in vesicle formation in the trans-Golgi network (TGN) and Chs6p mediated the cargo into the TGN-derived vesicles (Trautwein, Schindler et al. 2006). Chs3p-containing vesicles are later delivered to the plasma membrane in a polarized fashion. Chs $4 p$ has been recognized to promote Chs $3 p$ translocation into the plasma membrane in 
a stable and active form (Reyes, Sanz et al. 2007).

\section{The septation process:}

In fungi, cell division implies a formation of a cross wall, the septum, between the mouther and the daughter cells. The septation process in budding yeast is schematically illustrated in Figure 10. The position of the cell division plane, and that of the septum, is established at budding. The earliest structure to appear is the septin ring (Fig. 10A) that is formed at the incipient bud site before bud emergence and spreads into a hourglass structure after budding. The septin ring is a filamentous structure constituted by five proteins called septins, Cdc3p, Cdc10p, Cdc11p, Cdc12p and Shs1p (Gladfelter, Pringle et al. 2001; Castillon, Adames et al. 2003). This septin ring seems to serve as a scaffold for localization of proteins that participate in cell division. When the septin ring is formed, Chs $3 p$ forms a chitin disk at the basis of the site of bud emergence. After budding, the septin ring spreads into a hourglass structure. After completion of mitosis, the septin ring splits in two, leaving a gap in the middle (Fig. 10B). Chs2p appears in that gap (red dots in Figure 10). Then, as shown in Figure 10C, actomyosin ring contracts, pulling and invaginating the plasma membrane. Simultaneously, chitin synthesized by Chs $2 p$ is laid down in the evagination. The process continues with a centripetal deposition of chitin, forming a disk, the primary septum, while the contractile ring disappears and the membrane pinch off. At this point, cytokinesis has been completed, but not the septation process. Secondary septa are made with cell-material secreted through the membranes of both the mother and daughter cells (Fig. 10E).

The activity of two enzymes, a chitinase that digest part of the primary septum, and a $\beta$-(1,3)-glucanase, which degrades parts of the cell wall surrounding the septum, is required for the separation of the mother and daughter cells. Because the chitinase attacks the primary septum from the daughter cell side (Roberts, Bowers et al. 1983), most of the primary septum remains with the mother cell forming a crater-like structure, the bud scar (Cabib, 2004).

From different studies, as those from Roh and co-workers, it has emerged that the septation apparatus, composed of septins, an actomyosin contraction ring, and the chitin synthase system can function at ectopic locations autonomously and independently of cell division, and that it can recruit the other elements necessary for the formation of 
secondary septa (Roh, Bowers et al. 2002).

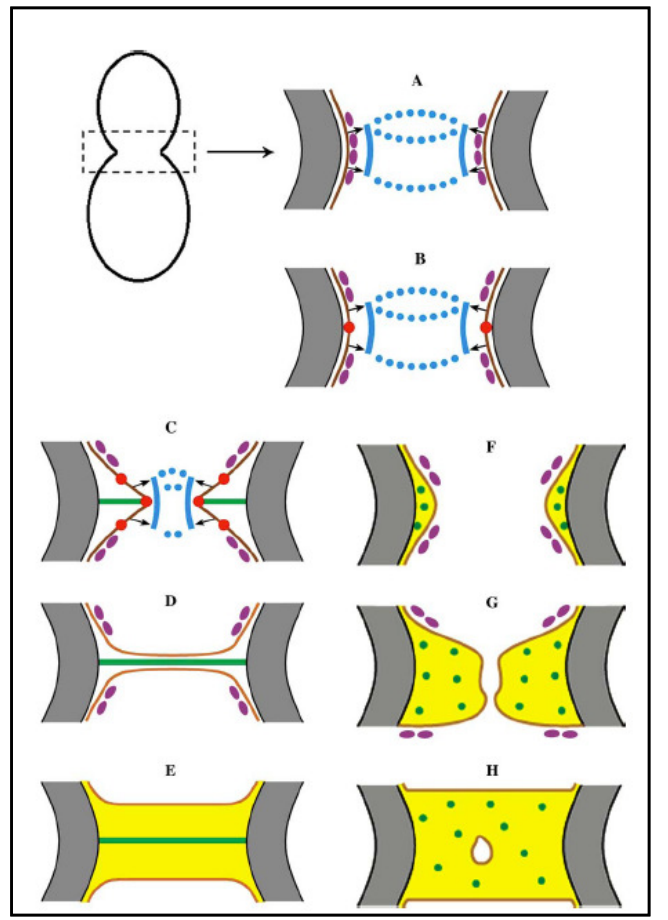

Figure 10. Scheme of the septation process in budding yeast. The cell wall is represented in grey, the plasma membrane in brown, the septin ring in purple, and the contractile ring in light blue. The septin ring, plasma membrane, and cell wall are shown in section. Only the contractile ring is drawn in perspective, to indicate the cylindrical shape of the neck. The small black arrows represent connections, of unknown nature, between contractile ring and plasma membrane.In (B) and (C), the red dots stand for Chs $2 \mathrm{p}$. In (C) to (H) green color represents chitin, either in the primary septum or dispersed in aberrant septa. (AE) Normal septation; $(\mathrm{F}-\mathrm{H})$ aberrant septation in chs2 and myo1 mutants. (from Cabib 2004).

\section{Chitin synthesis in C. albicans:}

In Candida albicans, the equivalent counterparts of ScCHS2, ScCHS1 and ScCHS3 are CaCHS1 (Au-Young and Robbins 1990), CaCHS2 (Chen-Wu, Zwicker et al. 1992) (Mio, Yabe et al. 1996) and CaCHS3(Sudoh, Nagahashi et al. 1993). It is known that both $\mathrm{CaCHS} 2$ and $\mathrm{CaCHS} 3$ are expressed preferentially during hyphal growth, whereas $\mathrm{CaCHSI}$ appears to be expressed in both yeast and hyphae at lower level (Chen-Wu, Zwicker et al. 1992; Munro, Schofield et al. 1998). CaChs1p displays the highest amino acid sequence identity with ScChs2p (ChenWu, Zwicker et al. 1992) and represents the orthologous enzyme. CaChs1p synthesizes the primary septal chitin and contributes to general cell wall integrity. CHS1 and is the only known CHS gene to date that has been shown to be essential for growth (Munro, Winter et al. 2001). The class I enzyme Chs2p represents the major chitin synthase activity in vitro. Null mutants of CHS2 had up to a $40 \%$ reduction in hyphal chitin, depending on the chitin assay used, and exhibited a marginal attenuation in virulence in a systemic mouse model of candidosis (Gow, Robbins et al. 1994; Munro, Schofield et al. 1998). Chs3p synthesizes the majority of chitin in yeast and hyphal cells (Bulawa, Miller et al. 1995). However, chs $3 \Delta$ null mutants have normal growth rates in vitro, near wild type morphology when grown under yeast or hypha-forming media and were attenuated in virulence despite being able to colonize the organs of infected mice (Bulawa, Miller et al. 1995).

In silico analysis of the genome sequence of $C$. albicans identified an open 
reading frame encoding a putative fourth member of the chitin synthase gene family. This gene was named CaCHS8 because the genes whose products encode post-translational regulators of Chs $3 \mathrm{p}$ had been named CHS4-7. The CHS8 gene product, like that of $C H S 2$, is a class I enzyme and $C$. albicans therefore differs from $S$. cerevisiae in having two isoenzymes in this class. Analysis of chs $8 \Delta$ performed by Munro and col. in wild type and chs $2 \Delta$ null mutants backgrounds demonstrated that $C$. albicans has four chitin synthases with two non-essential class I Chs isoenzymes that contribute collectively to more than $97 \%$ of the in vitro chitin synthase activity (Munro, Whitton et al. 2003).

In $C$. albicans the total specific activities of both chitin synthases and chitinases are higher in the hyphal form, mainly due to the activities of Chs $2 p$ and Cht3p (chitinase 3), respectively. It appears, that chitin synthesis and hydrolysis are not coupled, but that both are regulated during yeast-hypha morphogenesis (Selvaggini, Munro et al. 2004).

\subsubsection{Cell wall proteins:}

Mannoproteins of the yeast cell wall can be divided into four classes: 1) non covalently linked proteins, such as Bgl2p
(Klebl and Tanner 1989); 2) proteins that are linked covalently to the cell wall through disulfide bridges (Orlean, Ammer et al. 1986; Moukadiri, Jaafar et al. 1999); 3) Pir proteins, which are linked via an alkaline-sensitive bond to $\beta$-(1,3)-glucan (Kapteyn, Van Egmond et al. 1999; Jaafar, Moukadiri et al. 2003) and 4) GPIdependent cell wall proteins, which are covalently linked to $\beta$-(1,3)-glucan through $\beta$-(1,6)-glucan linked to a remnant of the GPI (Kapteyn, Montijn et al. 1996; Kapteyn, Ram et al. 1997; Kollar, Reinhold et al. 1997; Jaafar, Moukadiri et al. 2003). Cell wall proteins are processed through the secretory pathway, where they are $\mathrm{O}$-and often also N-glycosylated and where GPI is attached. N-glycosylated proteins receive a pre-formed oligosaccharide through an $\mathrm{N}$-glycosidic bond between a GlcNAc and an asparagine residue, while O-mannosylated proteins receive short mannose chains onto the hydroxyl side chains of serine or threonine residues through an $\alpha$-mannosyl bond.

Many fungal covalently linked cell wall proteins are involved in processes such as water retention, adhesion, and virulence. Many of these functions require proper glycosylation of the respective proteins. Mutations of the synthetic glycosylation and mannosylation pathways collectively affect all mannoproteins and cause 
multiple cell wall phenotypes, such as compound or stress hypersensitivity and altered cell wall composition. The proliferation of genes and gene products with overlapping functions in Golgi $\mathrm{N}$ and/or O-mannosylation suggests that such gene duplications have conferred fitness and have been selected during evolution (Lesage and Bussey 2006).

\section{THE GH72 FAMILY IN S. cerevisiae AND C. albicans :}

The Gel/Gas/Phr family of proteins from Aspergillus

fumigatus, Saccharomyces cerevisiae and Candida albicans plays an essential role in cell wall biogenesis acting as $\beta-(1,3)$-glucan processing enzymes (Mouyna, Fontaine et al. 2000). In vitro these proteins catalyze a $\beta$-(1,3)-glucanosyltransferase reaction that consists of the cleavage of an internal glycosidic linkage of a $\beta-(1,3)$-glucan chain, the release of the reducing end portion and the transfer of the new reducing end to the nonreducing end of another $\beta$-(1,3)-glucan that acts as an acceptor. Proteins related to the Gel/Gas/Phr family are widespread in yeast and fungal species and all together form the family $\mathrm{GH} 72$ in the carbohydrate active enzymes database (CAZy) of glycoside

hydrolases

(GHs)

(http://afmb.cnrs-

mrsm/fr/CAZY/fam/GH72.html) from the Pfam database

(http://www.sanger.ac.uk//cgi-

bin/Pfam/getacc??PF03198).

These proteins share common structural features: a secretory signal peptide at the $\mathrm{N}$ terminus and a carboxy terminal domain including both a Ser rich region and a GPI attachment signal, the latter being necessary for their anchoring to the plasma membrane. The central part of these proteins includes the catalytic domain $\mathrm{GH} 72$ responsible for their enzymatic activity a linker segment and a cysteine-rich domain, named Cysbox/CBM43 or $\mathrm{X} 8$ that is shared only by some members belonging of the GH72+ subfamily (Ragni, Fontaine et al. 2007).

Several studies have demonstrated that in A. fumigatus and C. albicans, strains carrying mutations in the homologs of these genes, belonging to the GEL and PHR gene families, show a great reduction in virulence in animal models of infection (De Bernardis, Muhlschlegel et al. 1998; Mouyna, Morelle et al. 2005). Because of the importance of this class of proteins in cell wall assembly and virulence, they are promising molecular targets for new antifungal drugs. Moreover, Gas proteins have been found to be fungal antigens. In a work to which 
our laboratory has participated in 2007, recombinant soluble forms of Gas1-5 from $S$. cerevisiae and their orthologous proteins Gel1-Gel2 from A. fumigatus have been found to be specifically recognized by antibodies present in the sera from patients with sperigillosis or candidiasis (Arroyo, Sarfati et al. 2007)
3.1. GAS MULTIGEN FAMILY OF $S$. cerevisiae:

The GAS multigene family of $S$. cerevisiae is constituted by five genes, GAS1-5. GAS family members play an essential role in cell wall assembly and remodelling acting as $\beta-(1,3)$ glucanosyltransferases.

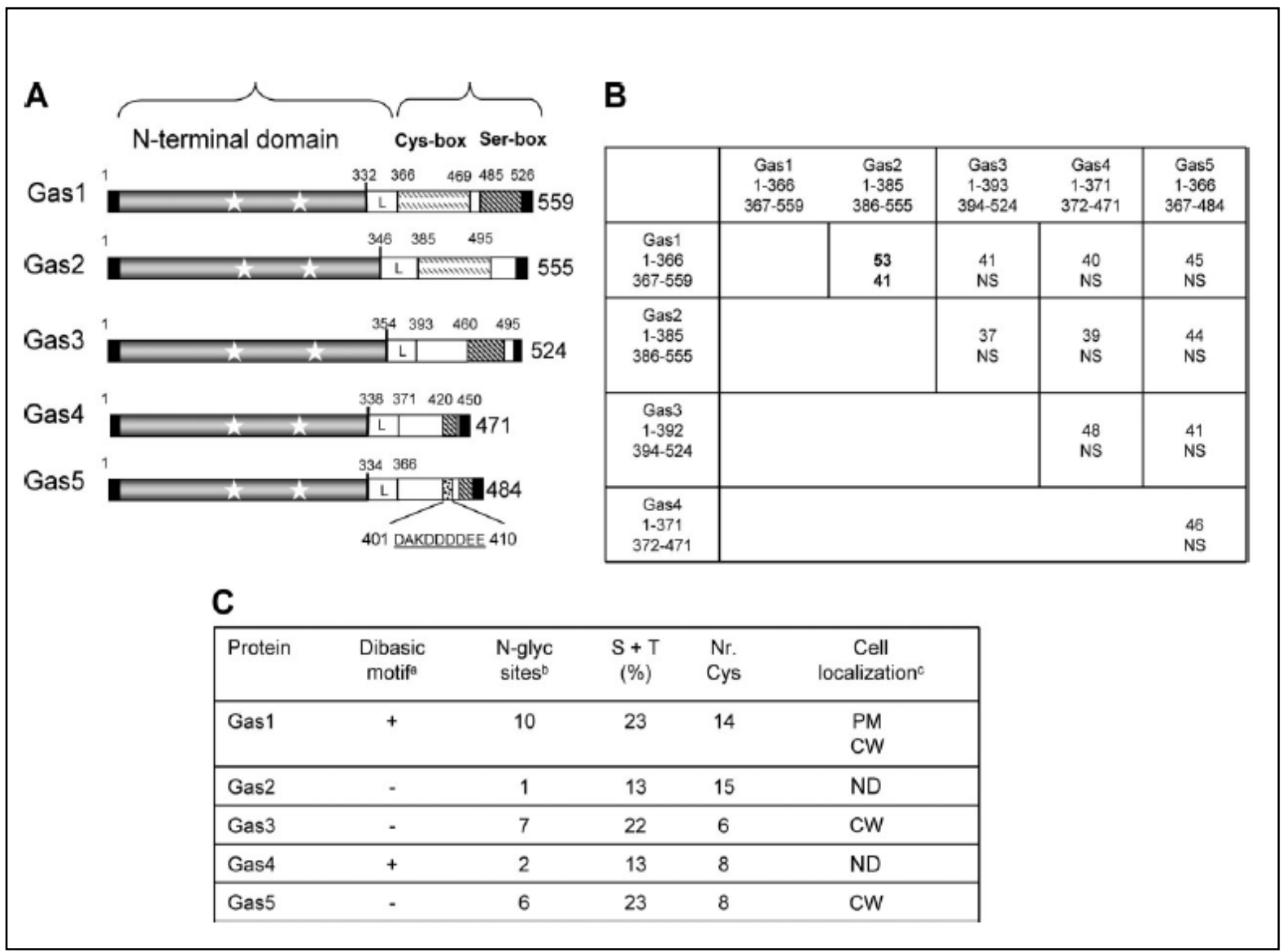

Figure 11. Relevant features of Gas proteins of S. cerevisiae. (A) Modular architecture of Gas proteins. The black boxes at the $\mathrm{N}$ - and the C-terminal are the signal peptide and the GPI-signal, respectively. The stars indicate the catalytic residues: E161 and E262 in Gas1p (Carotti et al., 2004), E176 and E275 in Gas2p, E169 and E283 in Gas3p, E161 and E266 in Gas4p and E160 and E262 in Gas5p. L is the putative linker segment. The Cys-box is the cysteine-enriched module, similar to CBM43, and the Ser-box is the serine-rich region. The short acidic region of Gas5p is also shown. (B) Percentage of amino acid identity among the N-terminal $(\mathrm{NtD}+\mathrm{L})$ regions or the $\mathrm{C}$-terminal regions of Gas proteins. The numbers indicate the first and last residue in the amino acid sequence of each region of a Gas protein. Values in bold: highest similarity; NS: no similarity. (C) Summary of some features of Gas proteins. (From Ragni, Fontaine et al. 2007). 
$G A S 1$, which is the best characterized member of the family, is expressed together with GAS5 exclusively during vegetative growth being repressed during meiosis and sporulation. The pair GAS2 and GAS4 shows the reverse pattern of expression, being induced during meiosis and sporulation and repressed during vegetative growth. $G A S 3$ is weakly expressed during vegetative growth and weakly induced during sporulation (Ragni, Coluccio et al. 2007).

Recently, the sequence of the five paralogs of Gas proteins has been extensively analysed in our laboratory. Figure 11A shows a schematic representation of the modular architecture of Gas proteins. They share a secretory signal sequence and an $\mathrm{N}$ terminal domain, named $\mathrm{NtD}$, which spans about 330-350 amino acids. The $\mathrm{NtD}$ is the putative catalytic domain, since it contains two glutamate residues that are conserved in all the Gas proteins and in all GH72 family members that have been shown to be essential for catalysis (Mouyna 2000) (Carotti, Ragni et al. 2004). This domain is annotated as Glyco_hydro_72 (GH72) in the Pfam database and was predicted to assume a TIM-barrel conformation (Papaleo, Fantucci et al. 2006). A putative linker segment, rich in flexible amino acids, such as T, G, A, P and S, and containing a highly conserved cysteine residue, is thought to connect the NtD to the $\mathrm{C}$ terminal region.

Figure 11B shows the percentage of amino acids identity in the $\mathrm{N}$-terminal $(\mathrm{NtD}+\mathrm{L})$ or the $\mathrm{C}$-terminal regions in the Gas protein family. The C-terminal region has a variable length and is highly different between the various Gas proteins except for Gas1p and Gas $2 p$ (see Figure 11B). Gas1p and Gas $2 p$ share a high identity in the C-terminal region $(41 \%)$, and this is due to the presence of domain named Cys-box, of about 100 amino acids. The Cys-box is also annotated as Pfam X8 in the Pfam Database. This domain contains a motif of six cysteine residues and, interestingly, it is conserved in some members of the family GH72 family and has a weak homology with a similar domain present in some plant $\beta-(1,3)$ glucanases of the family GH17, as well as in many other proteins of plant origin whose function is still unknown. This domain was recently classified as CBM43 in the carbohydrate binding modules (CBMs) database of $\mathrm{CaZy}$, since it was known to act as an autonomous non-catalytic module for binding to laminarin in the protein of olive pollen, Ole10 (Barral, Suarez, 2005) (Palomares 2003) (Palomares 
2005). In a recent work in which our laboratory has participated the Family GH72 was classified in two subfamilies, $\mathrm{GH}_{72}{ }^{+}$and $\mathrm{GH}^{-}$, that can be distinguished depending on the presence or absence of the C-terminal cysteinerich domain, the Cys-box (Ragni, Fontaine et al. 2007). Another study perfomed in our laboratory was focused on the characterization of the disulfide bridges in Gas1 and Gas2 proteins and led to the mapping of seven disulfide bonds, three in the $\mathrm{NtD}+\mathrm{L}$ region and four in the Cys-box/CBM43/X8 domain (Popolo, Ragni et al. 2008). In particular one of the bonds connects a central Cys residue of the $\mathrm{NtD}$ with a single conserved Cys residue in the linker. It has been demonstrated by site-directed mutagenesis that this relevant disulfide bond has a crucial role in folding as it may stabilize the NtD and facilitate its interaction with the $\mathrm{C}$-terminal portion of Gas proteins. Deletion of the Cys-box in Gas1 or Gas2 proteins leads to the formation of an $\mathrm{NtD}$ devoid of any enzymatic activity. These results suggest that the Cys-box is required for proper folding of the $\mathrm{NtD}$ and/or substrate binding in Gas1 and Gas2 proteins (Popolo, Ragni et al. 2008). These results were confirmed by a recent publication describing the 3D-structure of Gas2p of $S$. cerevisiae (HurtadoGuerrero, Schuttelkopf et al. 2009).

Moreover, at the end of the C-terminal region, a cluster of serine residues of variable length (Ser-box) is present is Gas1p, Gas3p, Gas4p and Gas5p but not in Gas2p. It is known that the Ser-box is not required for activity in Gas1p (Gatti, Popolo et al. 1994). Moreover, Gas5p contains a highly acidic sequence that could be part of an extended disorder segment of the protein, including a Ser box (Figure 11A).

Importantly, at the C-terminal end all proteins share a GPI-attachment signal (represented as a black box in Figure 10A). Gas1p and Gas4p contain a motif of two contiguous basic amino acids in the $\omega-1$ to $\omega-4$, where $\omega$ is the GPI attachment site (Figure 11C). This motif is a positive signal for the localization of the proteins in the plasma membrane (Caro, Tettelin et al. 1997; Frieman and Cormack 2003). Consistent with these data, several studies have shown that Gas1p is located in the plasma membrane (Popolo, Grandori et al. 1988; Conzelmann, Fankhauser et al. 1991). Gas2p or Gas $4 p$ are synthesized during meiosis and sporulation and localize to the spore periphery (Eleonora Rolli, $\mathrm{PhD}$ thesis, Jan 2009). Gas3 and Gas5 proteins are predicted to be preferentially anchored to the cell wall glucan network, 
given the absence of the dibasic motif in

their sequences although it is not known

whether the dibasic rule applies also to prospore membrane proteins. A comprehensive proteomic analysis of proteins covalently linked to the cell wall identified Gas3p and Gas5p as proteins attached to the cell wall via a GPI remnant (Yin, de Groot et al. 2005). Interestingly, in this mass spectrometry analysis as well as in other study (De Sampaio, Bourdineaud et al. 1999) Gas1p was also identified as cell-wall anchored. An analysis of Gas1 protein localization and in particular with regard to the presence of the protein in the cell wall will be presented in this thesis.

Interestingly, Gas1 protein has been reported to be associated to lipid rafts (Bagnat, Keranen et al. 2000). Lipid rafts are membrane microdomains enriched in sterols and sphingolipids. A distinctive feature of lipid rafts is their insolubility in mild non-ionic detergents (tipically Trition X-100) at $4^{\circ} \mathrm{C}$ (Brown, 1998) and therefore they are usually referred as detergent-resistant membranes (DRMs) or detergent-insoluble glycolipidenriched microdomains (DIGs) (Malinska, Malinsky et al. 2003). Lipid rafts are progressively enriched of their components along the secretory pathway and they are known to be required for the ER exit of several GPI-proteins. It has been proposed that rafts form a platform for lipid and protein sorting, trafficking and cell signalling (Malinska, Malinsky et al. 2003). Lipid rafts are well characterized in $S$. cerevisiae. Recently, together with Gas1p, also Gas3p and Gas5p have been found to be enriched specifically in the plasma membrane derived DRMs (Aronova, Wedaman et al. 2007).

\subsubsection{Gas $1 \Delta$ mutant phenotype:}

The absence of $\beta-(1,3)$ glucanosyltransferase activity of Gas1p causes an aberrant cell morphology and a transient cell wall damage. Cells lacking Gas1 protein shows a reduction in the growth rate of 15 to $40 \%$. gas $1 \Delta$ mutant cells are rounded, swollen, highly vacuolated and multibudded. Defects in bud maturation and cell separation are responsible to the appearance of what has been called "mickey mouse" morphology (Popolo, Vai et al. 1993). Cell wall properties are also affected: the permeability to external substances increases, cells becomes very sensitive to cell wall perturbing agents such as calcofluor white, the resistance to hydrolytic enzymes is high and similar to that of stationary growing cells (Ram, Wolters et al. 1994; Popolo, Gilardelli et al. 1997). All these defects reflect a 
series of modifications in the cell wall organization and composition. In fact, gas $1 \Delta$ mutation leads to a decrease in the incorporation of new glucan chains into the pre-existing cell wall and the crosslinking defects result in a release of mannoproteins in the culture medium (Ram, Wolters et al. 1994). The alkalisoluble/-insoluble glucan ratio shows a decrease and content in chitin increases (Popolo, Gilardelli et al. 1997; Valdivieso, Ferrario et al. 2000). This cell wall weakening caused by the absence of Gas1p induces a large fraction of genes that are devoted to cell wall reinforcement in order to ensure cell wall integrity. Damages in cell wall organization leads to the activation of the so called "integrity pathway", a MAP kinase pathway, that activates the transcription of cell wall proteins and enzymes involved in the cell wall synthesis and remodelling (Garcia, Bermejo et al. 2004).

\subsection{Phr PROTEIN FAMILY OF}

\section{Candida albicans:}

The first homologues of GAS genes in C. albicans that were described were the PHRI (Saporito-Irwin, Birse et al. 1995) and PHR2 genes (Muhlschlegel and Fonzi 1997). Phr1 and Phr2 proteins were found to be GPI-anchored cell surface proteins which process $\beta$-(1,3)glucans and create attachment sites for $\beta$-(1,6)-glucans (Fonzi 1999). Later on, new Gas1p homologoues of C. albicans, PHR3, PGA4 and PGA5 were described at the completion of the genome sequence by Eckert and col. (Eckert, Heinz et al. 2007).

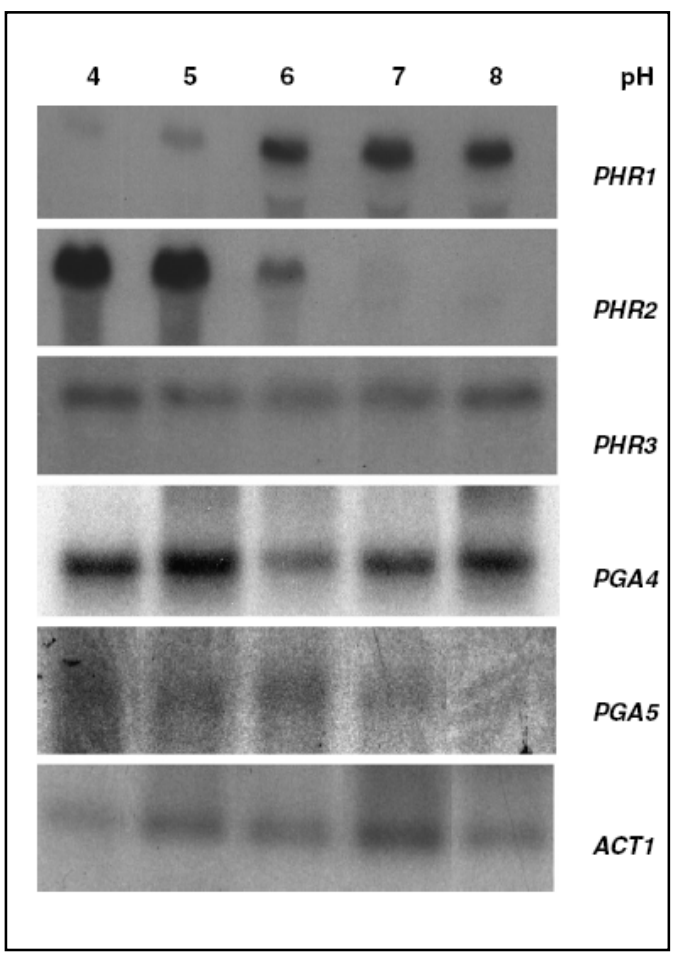

Figure 12. Expression of the $P H R$ genes in $C$. albicans. Total mRNA levels of PHR1, PHR2, PHR3, PGA4 and PGA5. Immunoblot of total mRNA isolated from SC5314 strains grown in liquid YNB medium at $\mathrm{pH}$ 4-8. (from Eckert, Heinz et al. 2007).

In vitro expression of PHRI is detected only when the ambient $\mathrm{pH}$ is above 5.5 and increases at more alkaline pH. PHR2 exhibits the inverse pattern, being expressed at valous 6 and with maximal expression levels below $\mathrm{pH} 5$ 
(Saporito-Irwin, Birse et al. 1995; Muhlschlegel and Fonzi 1997; Fonzi 1999). As shown in Figure 12, in contrast to $P H R 1$ and $P H R 2$, expression of the homologous PHR3, PGA4 and PGA5 genes has been found to be $\mathrm{pH}-$ independent. Expression of $P H R 3$ and PGA5 is constitutively low whereas PGA4 shows strong constitutive expression (Eckert, Heinz et al. 2007).

Either PHR1 or PHR2 are required for proper morphogenesis both in vitro and in vivo being both of them required for virulence. It has been demonstrated that the defects in virulence in animal models of infections of mutants lacking Phrlp or $\mathrm{Phr} 2 \mathrm{p}$ are $\mathrm{pH}$-dependent and reflect their pattern of expression (Ghannoum, Spellberg et al. 1995; De Bernardis, Muhlschlegel et al. 1998).

\subsubsection{PHR1 deletion phenotype and its role in virulence:}

Aberrant cell morphology is a characteristic phenotype of all mutants lacking PHR genes. As PHR1 and PHR2 were the first described members of the family and due to their role in virulence, mutants lacking both PHR1 and PHR2 have been extensively characterized. In particular I will focus in this thesis on Phr1 protein. As demonstrated by Fonzi et al. when PHRl gene was isolated, when growing at $25^{\circ} \mathrm{C}$, which promotes growth in the yeast morphology, the phrl null mutant exhibits morphological aberration becoming rounded and enlarged after extended growth at alkaline $\mathrm{pH}$ values such as at $\mathrm{pH} 7,5$ or 8. Shorter periods of incubation result in more subtle morphological changes. Rather than being ellipsoidal in shape, which is typical of normal buds, the buds of the mutant strain in restrictive conditions are rounder and flattener compared with buds of the parental strain. Despite these aberrant morphologies, the cells continued to exhibit polarized budding. It is important to remark that these morphological aberrations are not specific to the growth medium (Saporito-Irwin, Birse et al. 1995). PHRI null mutant presents also defects in hyphal growth, which that are also $\mathrm{pH}$-dependent. At $\mathrm{pH}$ 6.0, the phrls mutant was indistinguishable from the parental strain in both frequency and morphology of the germ tubes. At higher values of $\mathrm{pH}$, progressive increases in $\mathrm{pH}$ results in a corresponding reduction in the length of the germ tube and lateral expansion of the germ tube apex. At $\mathrm{pH}$ 8.0, the pattern culminates with the emergence of distorted growth projections, with many cells adopting aberrant shmoos and dumbbell-or 
peanut-shaped morphologies. This

behaviour of the mutant clearly demonstrates that the $\mathrm{pH}$ of the medium alters its ability to sustain or conduct apical growth in both the yeast and hyphal growth forms (Saporito-Irwin, Birse et al. 1995). PHR2 mutant exhibits the same aberrant phenotypes of PHRI but, since it is repressed at $\mathrm{pH}$ values above 6 and progressively induced at more acidic $\mathrm{pH}$ values, the mutant grows as the parental strain at values higher that 6 and it is unable to conduct apical growth at lower $\mathrm{pH}$ values (Muhlschlegel and Fonzi 1997).

PHR1 null mutant is avirulent is a mouse model of systemic infection but uncompromised in its ability to cause vaginal infection in rats. The virulence phenotype of $P H R 2$ null mutant is the inverse. Since systemic $\mathrm{pH}$ is near neutrality and vaginal $\mathrm{pH}$ is around 4.5, the virulence phenotype parallels the $\mathrm{pH}$ dependence of the in vitro defects that

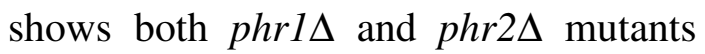
(Ghannoum, Spellberg et al. 1995; De Bernardis, Muhlschlegel et al. 1998).

\subsubsection{The $\mathrm{pH}$ response in}

\section{Candida albicans:}

The ability of $C$. albicans to respond to changes in extracellular $\mathrm{pH}$ is crucial for its survival in different environmental conditions and subsequently for its potential as a pathogen (Davis 2003).

A conserved signal transduction pathway that mediates adaptation to neutral-alkaline $\mathrm{pH}$ has been described in both the Ascomycestes, including $C$. albicans, and basidiomycetes (Davis, Wilson et al. 2000; Davis 2003; Kullas, Martin et al. 2007). This pathway is governed by the zinc finger DNAbinding protein Rim101/PacC. Rim101 binds to promoters and induces expression of genes expressed preferentially at acidic $\mathrm{pH}$ (Baek, Martin et al. 2006; Kullas, Martin et al. 2007). Rim101 activity is controlled by proteolytic removal of a C-terminal inhibitory domain ( $\mathrm{Li}$, Martin et al. 2004). In acidic environments Rim101 is either unprocessed ( $S$. cerevisiae) or processed to an inactive form $(C$. albicans) and in neutral-alkaline environments, Rim101 is processed to an active form ( $S$. cerevisiae and $C$. albicans). The processing event requires the upstream Rim101 pathway members, including Rim8/PalF, Rim13/PalB, Rim20/PalA, Rim21/PalH, Dfg16 and Snf7 (Futai, Maeda et al. 1999; Porta, Ramon et al. 1999; Davis, Wilson et al. 2000; Davis 2003; Li, Martin et al. 2004; Kullas, Martin et al. 2007). Importantly, proteolytic processing of Rim101 is 
critical for $C$. albicans pathogenesis in a murine model of heamatogenously disseminatated candidiasis (Davis, Edwards et al. 2000).

In C. albicans, Rim101p governs adaptation to neutral-alkalineenvironments by directly acting as an inducer and a repressor of gene expression (Davis 2003; Ramon and Fonzi 2003; Baek, Martin et al. 2006) whereas in $S$. cerevisiae Rim101p governs adaptation to neutral-alkaline environments primarily as a repressor (Lamb and Mitchell 2003). 
1. MICROORGANISMS USED IN THIS WORK:

\subsection{Bacteria strains and growth conditions:}

Escherichia coli strains used in this work are listed in Table 1.

Bacteria were grown at $37^{\circ} \mathrm{C}$ in LuriaBertani (LB) medium (1\% peptone, $0,5 \%$ yeast extract, $1 \% \mathrm{NaCl}$ and $2 \%$ agar for solid media) supplemented with antibiotics $(100 \mu \mathrm{g} / \mathrm{ml}$ of ampicilline).

\subsection{Saccharomyces cerevisiae strains and growth conditions:}

$S$. cerevisiae yeast strains used in this work are listed in Table 2.

Cells were grown in batches at $30^{\circ} \mathrm{C}$ in synthetic dextrose minimal medium (SD) (Difco yeast nitrogen base without amino acids at $6.7 \mathrm{~g} /$ liter, $2 \%$ glucose) to which amino acids and uracil were added to a concentration of $50 \mathrm{mg} / \mathrm{liter}$ and adenine to $100 \mathrm{mg} / \mathrm{liter}$, or in YPD (1\% yeast extract, 2\% Bacto-peptone, $2 \%$ glucose). For solid media $2 \%$ agar was added to SD media or YPD (YPDA and SDA).

Growth was monitored as the increase in optical density at $450 \mathrm{~nm}\left(\mathrm{~A}_{450 \mathrm{~nm}}\right)$. Duplication time (Td) was calculated by the equation $T_{d}=\mathrm{Ln} 2 / \mathrm{K}$, where $\mathrm{K}$, the growth rate constant, is the slope if the line obtained by linear regression on a semilogarithmic plot of the $\mathrm{OD}_{450 \mathrm{~nm}}$ values, whereas the growth rate, $\mu\left(\mathrm{h}^{-1}\right)$ was calculated as $1 / T_{d}$.

\subsection{1. $\alpha$-Factor treatment:}

Treatment with $\alpha$-factor $(\alpha-\mathrm{F})$ in $S$. cerevisiae was performed as follows. 20 $\mu \mathrm{g} / \mathrm{ml}$ of $\alpha-\mathrm{F}$ (GenScript Corp.) was added to cells from strains JC-9 and W303-1A, exponentially growing in YPD at $30^{\circ} \mathrm{C}$, at a cell density of about 5 X $10^{6}$ cells $/ \mathrm{ml}\left(\sim 0.3 \mathrm{OD}_{450 \mathrm{~nm}}\right)$, collected by centrifugation, washed with fresh YPD and suspended in fresh YPD at the same cell density. At different time intervals from $\alpha-F$ addition, aliquots corresponding to $13 \mathrm{OD}_{450}$ or $20 \mathrm{OD}_{450}$ were collected for the preparation of total extracts or for subcellular fractionation respectively.

\subsection{C. albicans strains and growth} conditions used in this work:

C. albicans strains used in this work are listed in Table 3.

\subsubsection{Candida albicans yeast growth:}

Candida albicans was routinely grown at $30^{\circ} \mathrm{C}$ in YPD $(1 \%$ yeast extract, $2 \%$ Bacto-peptone, 2\% glucose) or synthetic mimimal medium (SD) (Difco yeast nitrogen base without amino acids at 6.7 g/liter, $2 \%$ glucose and 
supplemented with the required amino acids or uridine). For Ura ${ }^{-}$strains all media, except those used for selection of Ura+ transformants were supplemented with $1 \mathrm{mM}$ uridine. When media with a defined $\mathrm{pH}$ was required, $150 \mathrm{mM}$ HEPES [4-(2-Hydroxyethyl) piperazine1-ethanesulfonic acid sodium salt; Sigma Aldrich] was added to the other components. Then the $\mathrm{pH}$ of the medium was adjusted to the required $\mathrm{pH}$ and the buffered medium was filter-sterilized. Solid medium was prepared as described above with the addition of $2 \%$ bactoagar.

\subsubsection{Induction of Candida albicans filamentation growth:}

To induce hyphal growth in liquid medium, yeast cells were cultured overnight to stationary phase at $25^{\circ} \mathrm{C}$ or $30^{\circ} \mathrm{C}$ in YPD or in YPD-150 mM HEPES buffered at $\mathrm{pH}$ 6. The stationaryphase cells were inoculated at a density of $2.5 \times 10^{6}-5 \times 10^{6}$ cells $/ \mathrm{ml}$ into prewarmed RPMI 1640 (Sigma Aldrich), $\alpha$ MEM [Alpha MEM w/o ribonucleosides, $\quad$ w/o deoxyribonucleosides, w/o NaHCO3, (Invitrogen)], Medium 199 [M199+ Earle's salts + L-Glutamine and w/o aminoacids (Gibco)], or YPD $+10 \%$ fetal calf serum (Difco) at $37^{\circ} \mathrm{C}$. All the inducing media were buffered with
$150 \mathrm{mM}$ HEPES to $\mathrm{pH} 7,7.5$ or 8 . Formation of germ tubes and hyphae was monitored by microscopy analysis after different time points (30min to $7 \mathrm{~h}$ ). Percentage of germ tube was calculated by counting the number of cells with a germ tube or with an elongated germ tube (hyphae). At least 200 cells were counted.

Filamentation on agar solid medium was assessed using Medium 199 plates solidified with $2 \%$ bacto-agar. Stationary-phase cells growing at $28^{\circ} \mathrm{C}$ were diluted to $\mathrm{OD}_{600 \mathrm{~nm}}=1.0$ in $1 \mathrm{~m}$ $\mathrm{dH}_{2}$. From that solution two $1: 100$ serial dilutions were made and from the last dilution 50 and $75 \mu \mathrm{l}$ were plated into Medium 199-150mM HEPES plates adjusted to the required $\mathrm{pH}$. Plates were incubated at $37^{\circ} \mathrm{C}$ for $4-5$ days.

\subsubsection{Down-shift of $\mathrm{pH}$ :}

Cells were grown at $25^{\circ} \mathrm{C}$ in YPD150mM HEPES buffered at pH 7.5, until $0 \mathrm{D}_{600}=0.5$. Then, cells were centrifuged and suspended in pre-warmed $\left(25^{\circ} \mathrm{C}\right)$ YPD-150mM HEPES buffered at $\mathrm{pH}$ 4.5. At time zero and after $30 \mathrm{~min}, 1 \mathrm{~h}, 3 \mathrm{~h}$ and $5 \mathrm{~h}$ aliquots of culture corresponding to $15 \mathrm{OD}_{600}$ were rapidly collected by filtration on nitrocellulose filters. Cells were resuspended in $800 \mu \mathrm{l}$ of ice-cold $\mathrm{dH}_{2} \mathrm{O}$, centrifuged for $2 \mathrm{~min}$ in a microfuge at the maximum speed and 
then the pellet was quickly frozen in dryice acetone and stored at $-20^{\circ} \mathrm{C}$ for subsequent preparation of total protein extracts. At each time point $2 \mathrm{ml}$ of cells were sonicated, collected by mild centrifugation (2 $\mathrm{min}$ at 8,000 r.p.m.), washed twice with cold PBS and kept on ice for fluorescence microscopy analysis. In addition, at $\mathrm{T}_{\mathrm{O}}$ and $5 \mathrm{~h}$ aliquots of culture corresponding to 20 $\mathrm{OD}_{600}$ were harvested and processed for the isolation of the cell walls. At $5 \mathrm{~h}$ after the $\mathrm{pH}$ down-shift the medium was also collected and frozen at $-20^{\circ} \mathrm{C}$ until the following day. Precipitation of the proteins from the medium was performed in $10 \%$ trichloroacetic acid (TCA) in ice for 1h. After centrifugation $(10 \mathrm{~min}$ at $13,000 \mathrm{x} \mathrm{g})$ at $4^{\circ} \mathrm{C}, 3 \mathrm{ml}$ of cold acetone were added to the pellet whereas the supernatant was discarded. Samples were centrifuged again for $10 \mathrm{~min}$ at $13,000 \times \mathrm{g}$ at $4^{\circ} \mathrm{C}$ the pellet was let evaporate. The surpernatant was discarded and he pellet was let evaporate. After that, the sample was denatured in SDS- sample buffer at $95^{\circ} \mathrm{C}$ for $5 \mathrm{~min}$ and neutralized by addition of $1 \mathrm{M}$ Tris.

\section{LIST OF BACTERIA AND YEAST STRAINS USED IN THIS STUDY}

\begin{tabular}{|c|c|c|}
\hline Strain & Genotype & Source \\
\hline $\mathrm{DH} 5 \alpha$ & $\begin{array}{l}\text { F'/endA1 hsdR17(rk m }^{-} \mathrm{k}^{-} \text {) supE44 thi-1 recA1 gyrNAl } \\
\text { relA1A(lacZYA-orfF) U169 deoR( } \$ 80 \text { dlacD (lacZ)M15) }\end{array}$ & INVITROGEN \\
\hline TOP & 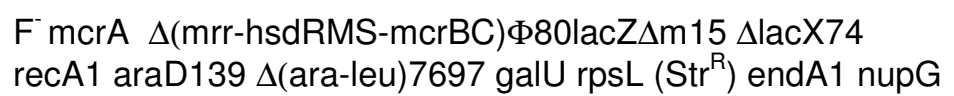 & INVITROGEN \\
\hline
\end{tabular}

Table 1. List of bacteria strains used in this study

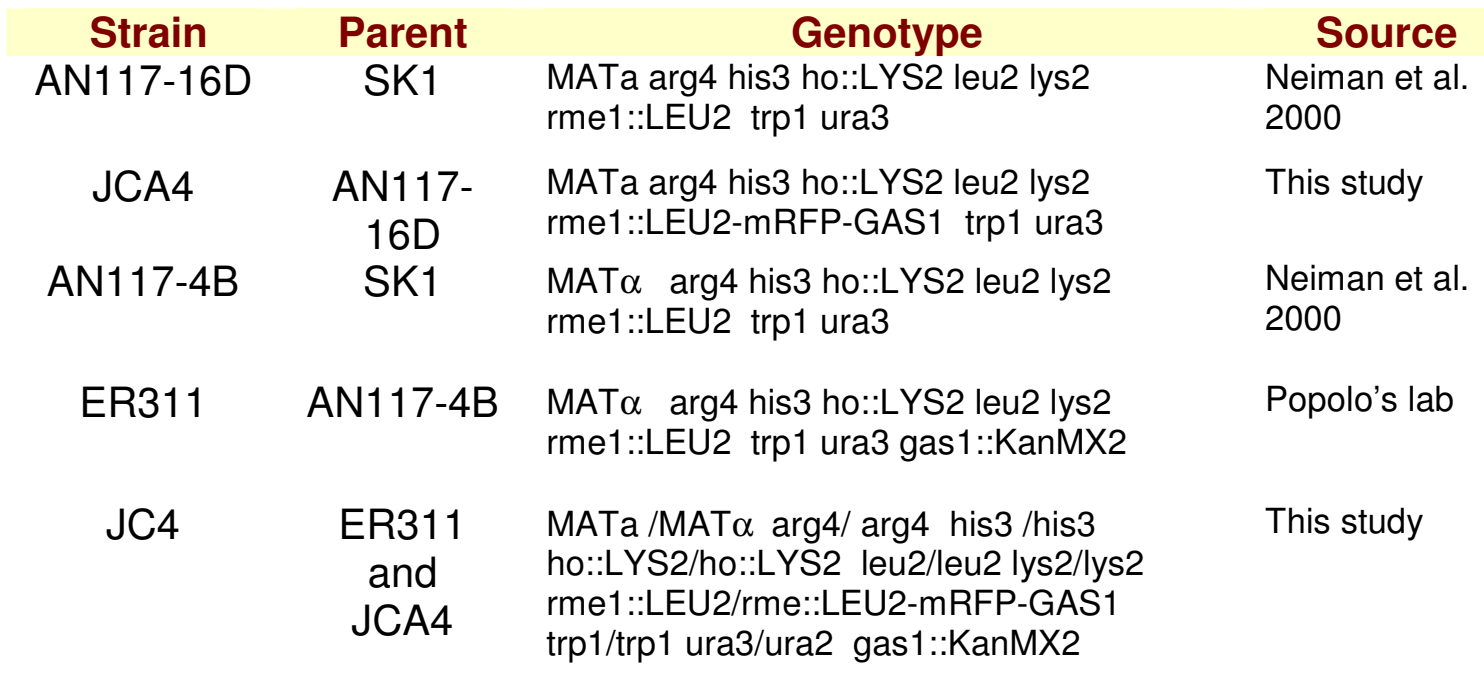

Table 2. S. cerevisiae strains used in this study 


\begin{tabular}{|c|c|c|c|}
\hline EC4 & $\begin{array}{l}\text { AN117-4B } \\
\text { and } \\
\text { JCA4 }\end{array}$ & $\begin{array}{l}\text { MATa /MAT } \alpha \text { arg4/ arg4 his3 /his3 } \\
\text { ho::LYS2/ho::LYS2 leu2/leu2 lys2/lys2 } \\
\text { rme1::LEU2/rme::LEU2-mRFP-GAS1 } \\
\text { trp1/trp1 ura3/ura2 }\end{array}$ & This study \\
\hline W3031A & W303 & $\begin{array}{l}\text { MATa ade } 2-1 \text { his3-11,15 trp1-1 ura3-1 } \\
\text { leu2-3,112 can } 1-100\end{array}$ & \\
\hline JC5 & W3031A & $\begin{array}{l}\text { MATa ade2-1 his3-11,15 trp1-1 ura3-1 } \\
\text { leu2-3-mRFP-GAS1,112 can1-100 }\end{array}$ & This study \\
\hline WAH & W3031A & $\begin{array}{l}\text { MATa ade } 2-1 \text { his3- } 11,15 \text { trp1-1 ura3-1 } \\
\text { leu2-3,112 can } 1-100\end{array}$ & \\
\hline JC9 & WAH & $\begin{array}{l}\text { MATa ade2-1 his3- } 11,15 \text { trp1-1 ura3-1 } \\
\text { leu2-3-mRFP-GAS1,112 can1-100 }\end{array}$ & This study \\
\hline
\end{tabular}

Table 2. S. cerevisiae strains used in this study (continuation)

\begin{tabular}{|c|c|c|c|}
\hline & Parent & Genotype & \\
\hline SC5314 & & Clinical isolate & $\begin{array}{l}\text { Kirsch et al. } \\
1984\end{array}$ \\
\hline CAF3-1 & $\begin{array}{c}\text { SC531 } \\
4\end{array}$ & Aura3::imm434/_ura3::imm434 & $\begin{array}{l}\text { Fonzi et al. } \\
1995\end{array}$ \\
\hline $\begin{array}{l}\text { CT1 or } \\
\text { JC9.2 } \\
\text { (cl } 9.2 \text { ) }\end{array}$ & CAF3-1 & $\begin{array}{l}\text { Aura3::imm434/_Lura3::imm434 } \\
\text { PHR1/PHR1-GFP }\end{array}$ & This study \\
\hline $\begin{array}{c}\text { CT2 } \\
\text { (cl.9.4) }\end{array}$ & CAF3-1 & $\begin{array}{l}\text { Aura3::imm434/_Lura3::imm434 } \\
\text { PHR1/PHR1-GFP }\end{array}$ & This study \\
\hline $\begin{array}{c}\text { CT3 } \\
\text { (cl 9.5) }\end{array}$ & CAF3-1 & $\begin{array}{l}\text { Aura3::imm434/_Aura3::imm434 } \\
\text { PHR1/PHR1-GFP }\end{array}$ & This study \\
\hline $\begin{array}{l}\text { UBP8 } \\
\text { (cl } 8 \text { ura+) }\end{array}$ & CT2 & PHR1-GFP Aura3::imm434/pLUBP-URA3 & This study \\
\hline $\begin{array}{l}\text { UBP10 } \\
\text { (cl 10 } \\
\text { ura+) }\end{array}$ & CT2 & PHR1-GFP $\Delta u$ ra3::imm434/pLUBP-URA3 & This study \\
\hline CAI10 & CAF3-1 & URA3/Aura3::imm434 & $\begin{array}{l}\text { Fonzi et al. } \\
1998\end{array}$ \\
\hline CAS10 & CAS8 & 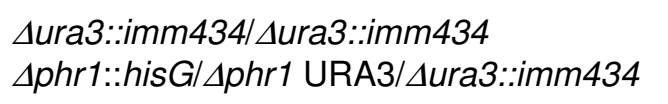 & $\begin{array}{l}\text { Fonzi et al. } \\
1998\end{array}$ \\
\hline CAS11 & CAS8 & $\begin{array}{l}\text { Aphr1::hisG/PHR1-pUC18-URA3- } \Delta p h r 1 \\
\text { Aura3::imm434/Aura3:::imm434 }\end{array}$ & $\begin{array}{l}\text { Fonzi et al. } \\
1998\end{array}$ \\
\hline
\end{tabular}

Table 3. C. albicans strains used in this study 


\section{DNA MANIPULATION}

\section{TECHNIQUES:}

\subsection{Isolation of DNA:}

\subsubsection{Preparation of plasmid DNA:}

To extract plasmid DNA from bacterial cell suspensions, QIAprep Spin Miniprep Kit or QIAGEN Plasmid Midi Kit (QIAGEN) were used. Both Miniprep and Midipreps were done using a microfuge following the instructions of the commercial brand. Plasmid purification using QIAprep Kits follows a simple bind-wash-elute procedure. First, bacterial cultures are lysed and the lysates are cleared by centrifugation. The cleared lysates are then applied to the QIAprep module where plasmid DNA adsorbs to the silicagel membrane. Impurities are washed away and pure DNA is eluted in a small volume of elution buffer or water.

\subsubsection{Total DNA extraction from yeast:}

Yeast cells were grown overnight in $10 \mathrm{ml}$ of $\mathrm{YPD}$ at $30^{\circ} \mathrm{C}$ under vigorous agitation. The following morning cells were collected by centrifugation and resuspended in $200 \mu \mathrm{l}$ of SZ buffer (for 25ml: $6.8 \mathrm{ml} \mathrm{H}_{2}$ 0, $12.5 \mathrm{ml}$ Sorbitol, 2.5 $\mathrm{ml} 1 \mathrm{M}$ NaCitrate, $3 \mathrm{ml}$ 0.5M EDTA $\mathrm{pH}$ 8, 15mg Zymolyase 20T, 0.2ml $\beta$ - mercaptoethanol) and incubated for 1hour at $37^{\circ} \mathrm{C}$. Treatment with Zymolyase (a $\beta(1,3)$-glucanase) serves to digest the cell wall. Spherophlasts were centrifugated for $2 \mathrm{~min}$ at 2,000 rpm and, after discarding the supernatant, the pellet was resuspended in $200 \mathrm{ml}$ of SDS-TE (2\% SDS, $0.1 \mathrm{M}$ Tris-HCl $\mathrm{pH} \quad 9.0, \quad 0.01 \mathrm{M}$ EDTA). Incubation was performed at $65^{\circ} \mathrm{C}$ for 15 min. Subsequently, $200 \mu \mathrm{l}$ of $5 \mathrm{M}$ KAcetate were added and samples were incubated at $4^{\circ} \mathrm{C}$ for 1 hour. After centrifugation at $13,000 \mathrm{rpm}$ at $4^{\circ} \mathrm{C}$ for 10 min the supernatants were transferred into new Eppendorf tubes. $1 \mu \mathrm{l}$ of isopropanol and $200 \mathrm{ml}$ of $\mathrm{NH}_{4}$ Acetate were added and samples were incubated at $-20^{\circ} \mathrm{C}$ for $30 \mathrm{~min}$ to precipitate nucleic acids. After 15 min-centrifugation at $13,000 \mathrm{rpm}$ at $4^{\circ} \mathrm{C}$, the pellet constituted by DNA and RNA was washed with $1 \mathrm{ml}$ of EtOH $70 \%$. Finally the pellet was dried in Savant and $20 \mu \mathrm{l}$ of TE+ RNAse were added to eliminate RNA by further 10 min incubation at $37^{\circ} \mathrm{C}$.

\subsection{DNA amplification by PCR:}

For the amplification of DNA fragments the Polymerase Chain Reaction was used. The multiple cycles of heating and cooling involved in PCR were performed using an Eppendorf 
Mastercycler thermocycler. DNA polymerase from Thermus aquaticus, Taq polymerase, was used. Taq polylmerase enzymes from Fermentas or Invitrogen were used for routine PCR reactions. For amplifications in which high fidelity was required Expand we used the Expand High Fidelity PCR system (Roche). Expand Hi Fi DNA Polymerase is composed of a unique enzyme mix containing thermostable Taq DNA polymerase and Tgo DNA polymerase, a thermostable DNA polymerase, the latter being a DNA polymerase with proofreading activity.

Conditions used for PCR reactions were set up according to the indications described in the datasheet of the enzymes and following the basic protocols described in the molecular biology manuals.

To perform several parallel reactions, master mix was prepared containing sterile MilliQ water, buffer, $\mathrm{MgCl}_{2}$, dNTPs, primers and Taq DNA Polymerase in a single tube, which was aliquoted into individual tubes. Template DNA solutions were then added to each tube. This method of setting reactions minimizes the possibility of pipetting errors and saves time by reducing the number of reagent transfers

\subsection{DNA Gel electrophoresis:}

Conventional electrophoresis in agarose gels was used to visualize the DNA, to quantify it or to isolate a particular DNA fragment. Electrophoresis was carried out in proper electrophoresis chambers and using a power supply. DNA was stained by addition of ethidium bromide stained and DNA bands were visualized under UV light by use of with a transiluminator.

To determine the size of under UV light by use of DNA fragments, Molecular Weight Markers were used. We used Gene Ruler 1kb DNA ladder as well as a Mass Ruler DNA ladder when quantification was required (Fermentas).

\subsection{Purification of PCR products:}

PCR products were routinely desalted and purified by salt/ethanol precipitation. An aliquot corresponding to $1 / 10$ of the sample volume of NaAcetate $3 \mathrm{M}$ pH 5.4 and 2.5 volumes of ice-cold ethanol $96 \%$ were added to the PCR reactions which were then incubated at $-20^{\circ} \mathrm{C}$ overnight. The following morning, the sample was centrifuged at 13,000 rpm and the pellet was washed with $1 \mathrm{ml}$ of ice-cold $\mathrm{EtOH}$ $70 \%$. DNA was finally dried in Savant, suspended in 5-20 $\mu \mathrm{l}$ of TE buffer and stored at $-20^{\circ} \mathrm{C}$ until use. 
In the case in which a complete removal of contaminants and inhibitors was needed GENECLEAN Turbo Kit for PCR (Qbiogene) was used.

\subsection{Isolation of DNA fragments from agarose gels:}

When required, DNA fragments were purifed from agarose gels. We started by excising the desired band using a razor blade from an ethidium-stained gel viewed with a UV transilluminator. The piece of agarose gel containing the DNA was then processed with a QIAquick Gel Extraction Kit (Quiagen). QIAquick Gel Extraction Kit provides spin columns, buffers and collection tubes for silica-membrane-based purification of DNA fragments from gels.

\subsection{Isolation of DNA fragments from agarose gels:}

Restriction enzymes function under specific conditions of salt concentration, temperature and $\mathrm{pH}$. Therefore the reactions are performed in a buffer solution at a defined temperature. Restriction enzymes are supplied with concentrated buffer to make this buffered solution. We used enzymes from NEB or Roche. To be sure that we were using the buffer that was optimal for the enzyme (s) with which we were working, we followed the recommendations from the datasheet of the products or from technical information which were available on the websites.

\section{TRANSFORMATION OF BACTERIA AND YEAST WITH EXOGENOUS DNA:}

\subsection{E. coli transformation:}

E. coli competent cells were routinely obtained in the laboratory. Competent cells were aliquoted and freezed at $-80^{\circ} \mathrm{C}$ into labelled screw-cap microcentrifuge tubes until use.

Transformation of plasmid DNA to competent E. Coli cells was done as follows. $100 \mu \mathrm{l}$ of competent cells were thawed on ice and then $2 \mu$ l of plasmid were added. After gently vortex the tubes were putted on ice for $30 \mathrm{~min}$. The cells were then heat shocked at $42^{\circ} \mathrm{C}$ for 2 min and immediately placed on ice of at least $2 \mathrm{~min}$. After addition of $250 \mu \mathrm{l}$ of SOC medium (2\% Tryptone, $0.5 \%$ Yeast Extract, $10 \mathrm{mM} \mathrm{NaCl}$, $10 \mathrm{~m} \mathrm{MMgSO}_{4}, 10 \mathrm{mM} \mathrm{MgCl}_{2}$ ) tubes were incubated for 1 hour at $37^{\circ} \mathrm{C}$. Tubes were shaken vigorously and spun down briefly. Supernatant was removed and the pellet was resuspended in SOC medium. The suspension was plated out on LB agar plates (supplemented with 
Ampicillin) and incubated overnight at $37^{\circ} \mathrm{C}$.

\subsection{Saccharomyces cerevisiae}

\section{transformation:}

Lithium acetate protocol adapted from (Gietz, Schiestl et al. 1995) was used to transform Saccharomyces cerevisiae cells with exogenous DNA. Yeast cells were inoculated in $10 \mathrm{ml}$ of YPD and grown overnight at $30^{\circ} \mathrm{C}$. The following day, cells were inoculated in $50 \mathrm{ml}$ of fresh rich medium at a cell density about $5 \times 10^{6}$ cells $/ \mathrm{ml}$ and let grow until reaching a cell density of $2 \times 10^{7}$ cells $/ \mathrm{ml}\left(\mathrm{OD}_{450 \mathrm{~nm}}\right.$ about1-1.5). Cells were harvested at $4000 \mathrm{rpm}$ for $5 \mathrm{~min}$, washed once with $\mathrm{dH}_{2} \mathrm{O}$ and resuspended in $1 \mathrm{ml}$ of $100 \mathrm{mM}$ lithium acetate. The solution was removed after a short centrifugation at top speed for $5 \mathrm{sec}$ and cells were resuspended to a final volume of $500 \mu 1$ of $100 \mathrm{mM}$ lithium acetate. $50 \mu \mathrm{l}$ of the cell suspension were pelleted to remove the solution. The transformation mix was prepared by sequential addition of: $240 \mu \mathrm{l}$ of polyethylenglycol (PEG) (50\% w/v), $36 \mu \mathrm{l}$ of $1 \mathrm{M}$ lithium acetate, $25 \mu \mathrm{l}$ of single strand carrier salmon DNA (2mg/ml), 50ml of $\mathrm{dH}_{2} \mathrm{O}$ and plasmid DNA $\quad(0.1-10 \mu g)$. After vigorous shaking, the mix was incubated for $30 \mathrm{~min}$ at $30^{\circ} \mathrm{C}$ and then subjected to a heat shock for $25 \mathrm{~min}$ at $42^{\circ} \mathrm{C}$. Cells were collected at $8000 \mathrm{rpm}$ for $15 \mathrm{~s}$ to remove the transformation mix and resuspended in $500 \mu \mathrm{l}$ of $\mathrm{dH}_{2} 0$. Different dilutions of the sample were spread on selective plates and let grown for at least 3 days in an incubator at $30^{\circ} \mathrm{C}$.

\subsection{Candida albicans transformation protocol:}

Candida albicans was transformed using a modified version of the lithium acetate procedure. Cells were inoculated in 10ml YPD and incubated overnight at $30^{\circ} \mathrm{C}$ until stationary phase. Cells were then diluted to $\mathrm{OD}_{600 \mathrm{~nm}}=0.1-0.2$ and incubated at $30^{\circ} \mathrm{C}$ with shaking until a final $\mathrm{OD}_{600 \mathrm{~nm}}$ of approximately 0.8-1.0. Cells were harvested for $10 \mathrm{~min}$ at 4,000 rpm, washed once with sterile $\mathrm{dH}_{2} \mathrm{O}$ and resuspended in $1.5 \mathrm{ml}$ of $100 \mathrm{mM}$ of LiAc-TE buffer (100mM LiAc, 100mM Tris-HCl pH 7.5, 1mM EDTA). Cell were then pelleted at top speed for $5 \mathrm{~s}$. $100 \mu \mathrm{l}$ of C.albicans competent cells were transferred to an Eppendorf tube and then 1-10 $\mu \mathrm{g}$ DNA and $100 \mu \mathrm{g}$ of single strand DNA were added. To this transformation mixture, $600 \mu \mathrm{l}$ of PEG/LiAc solution (50w/v polyethylenglycol in LiAc-TE buffer) was added and briefly vortexed. 
Subsequently, the transformation mixture was incubated for 20-24 hours at $30^{\circ} \mathrm{C}$. Cells were then pelleted and resuspended in $250 \mu \mathrm{l}$ of sterile $\mathrm{dH}_{2} \mathrm{O}$, plated onto selective plates and incubated for up to 4 days prior to counting the transformants.

\section{CONSTRUCTION OF $S$. cerevisiae AND C. albicans FUSION PROTEINS:}

\subsection{Construction of $S$. cerevisiae strain expressing mRFP-GAS1 fusion protein:}

WAH (gas1 $\Delta$ ) strain was transformed with the integrative plasmid pMF608, a kind gift of Prof. Y. Jigami (AIST, Tokyo). Plasmid pMF608 harbours an mRFP-GAS1 fusion under the control of the natural GAS1 promoter. The Nterminal sequence of the encoded hybrid protein is MLFKSLSKLATAAAFFAGVATA $\downarrow D \underline{\mathrm{TR}}$ ASASSE - where the signal peptide of Gas1p is in italics, the amino acids of the linker are underlined, the first four aminoacids of mRFP are in bold and the arrow indicates the cleavage site for leader peptidase. Plasmid pMF608 was linearized by digestion with $B f r \mathrm{I}$ for targeting into the LEU2 locus. $\mathrm{Leu}^{+}$ transformants were analyzed for the presence of the fluorescent protein. Strain JC9 (Table 2) was obtained and further characterized

\subsection{Construction of $C$. albicans strain expressing Phr1-GFP fusion protein:}

For the construction of a chimera of Phrlp with the green fluorescent protein (GFP), an internal tagging was necessary (see Chapter II). By using a GlobPlot prediction (http://globplot.embl.de/), two disorder regions were selected for the tagging. Both an $\mathrm{N}$-terminal and a Cterminal tagging were obtained. The $\mathrm{N}$ terminal insertion site was between aminoacids S24-S25 whereas in the Cterminal region was between the residues G489 and G490.

By using a previously reported PCRbased strategy we amplified the GFPURA3-GFP cassette from pGUG plasmid (kindly gifted by Cheryl Gale) with two different pairs of primers for the $\mathrm{N}$-terminal and C-terminal taggings. The primers used for the N-terminal insertion were: SSFOR: GTATTCATTAATCAAATCATTGGT TACATTTGCCACACTCTTTTCATTA ACTTTAGCCAAGTTTGAATCGTCT AAAGgTGAAGAATTATTCACTG G and SSREV: GTATTCATTAATCAAATCATTGGT TACATTTGCCACACTCTTTTCATTA 
ACTTTAGCCAAGTTTGAATCGTCT

\section{AAAGgTGAAGAATTATTCACTG}

G (in bold: amino acids corresponding to the GFP sequence) whereas the ones used for the C-terminal tagging were: GPFOR2:

TGATTTCAAAGGCAGTGCTTCAAT CAATATCAAGGCTAGTGCTAGTGG CAGCTGCAAAGCTGTTAGTGGAGT AGCTACTGGTAAGGCATCTTCCTC TGGTGGTGGTTCTAAAGGTGAAG AATTATTCACTGG -where the underlined nucleotides encode for two glycan residues (linker amino acids)and:

\begin{tabular}{|c|c|}
\hline $\begin{array}{l}\text { Name } \\
\text { SSFOR }\end{array}$ & $\begin{array}{l}\text { Sequence } \\
\text { GTATTCATTAATCAAATCATTGGT7 } \\
\text { TCATTAACTTTAGCCAAGTTTGAA } \\
\text { TTATTCACTGG }\end{array}$ \\
\hline SSREV & $\begin{array}{l}\text { TGATTAAAAACTGAGACCCGTTAT } \\
\text { TACCAACAACTTCAACTGGTGGAC } \\
\text { CCATAC }\end{array}$ \\
\hline GPFOR2 & $\begin{array}{l}\text { TGATTTCAAAGGCAGTGCTTC } \\
\text { GTGCTAGTGGCAGCTGCAAAG } \\
\text { CTACTGGTAAGGCATCTTCCT } \\
\text { AGGTGAAGAATTATTCACTGG }\end{array}$ \\
\hline GPREV2 & $\begin{array}{l}\text { GAGTTGCTTTAACTCCAGAGC } \\
\text { GCTGGTGCTGCTGCTTGATGA } \\
\text { GAGGAAGATCCAGATTTGGAG } \\
\text { CATCCATAC }\end{array}$ \\
\hline PHRFOR & ACATACCAAACTACAGGTTG \\
\hline GFREV & TCATATGATCTGGGTATCTA \\
\hline URA3FOR & GAATGGAGTGGTTGAAGGAT \\
\hline URA3REV & АТССТТСААССАСТССАТТС \\
\hline GFFOR2 & CAAATTGGAATACAACTATA \\
\hline GFREV2 & TGTCTGGTAACAAGACTCGA \\
\hline
\end{tabular}

Table 3. C. albicans strains used in this study
GPREV2:GAGTTGCTTTAACTCCAG AGCTTGAGCTGGACCCAGAGCTG GTGCTGCTGCTTGATGATCCAGAA GTAGATGCAGAGGAAGATCCAGA TTTGGAGCTTCCTTTGTACAATTC ATCCATAC. For all the primers the 5' segments (80nt for SSFOR2 and SSREV2; and 100nt for GPFOR2 and GPREV2) were homologous of PHR1 sequence in the site selected for the insertion and the last ones (in bold) were complementary to the GFP sequence at both ends of the construct in the PCR template plasmid. 
The PCR products amplified in this way were used to transform CAF3-1 strain. Transformants were then checked by PCR for correct integration of the cassette. The excision of the URA3 marker was performed as follows. $5 \mathrm{ml}$ of cells were grown in YPD at $25^{\circ} \mathrm{C}$ for $24 \mathrm{~h}$ and then $100 \mu \mathrm{l}$ of the culture were transferred into fresh YPD medium and incubated again for 24. After that cells were washed twice and resuspended in $\mathrm{dH}_{2} \mathrm{O}$. After measurement of $\mathrm{OD}_{600 \mathrm{~nm}}$, $10^{6}$ and $5 \times 10^{6}$ cells were plated onto 5FOA plates $(\mathrm{YNB}+625 \mu \mathrm{g} / \mathrm{ml}$ 5-FOA). Excision of $U R A 3$ was also checked by PCR.

5.

MICROSCOPY

\section{TECHNIQUES:}

\subsection{Light microscopy:}

Cells were routinely observed by phase-contrast microscopy using an Olympus BX60 microscope and a DC290 Kodak digital photo camera or with a Leica. Cells were scored for budding or filamentation (in C. albicans) by counting at least 200 cells after mild sonication.

\subsection{Fluorescence microscopy:}

For fluorescence microscopy cells were either fixed or analyzed without fixation. In either case cells were sonicated before being processed. For direct GFP visualization cells were washed three times with PBS by 2 mincentrifugation at 2,000 rpm. After the washes, cells were resuspended in $1 \mathrm{~m}$ of PBS and incubated for at least 15 min on ice before examination under the microscope. For visualization of fixed cells, about $2 \times 10^{8}$ cells were treated with $3.7 \%$ formaldehyde and $0.1 \mathrm{M} \mathrm{K-}$ phosphate $\mathrm{pH} 6.5$ for $30 \mathrm{~min}$ at room temperature. Cells were filtered and resuspended in the same volume of a fixing buffer [3.7\% formaldehyde, 0.1 M K-phosphate, $\mathrm{pH}$ 6.5]. Cells were washed twice with PBS, pH 7.4 at $4{ }^{\circ} \mathrm{C}$ and left in PBS for $1 \mathrm{~h}$ in ice before examination under the microscope. The cells were examined as wet mounts using an Olympus BX60 microscope and a DC290 Kodak digital photo camera or a Leica DMRA2 microscope at the Centro Interdipartimentale di Microscopia Avanzata dell'Università degli Studi di Milano C.I.M.A. 


\subsection{Confocal microscopy:}

For confocal microscopy cells were examined using a Leica TCS SP2 AOBS (Leica Microsystems, Heidelberg, Germany) confocal laser-scanning microscope, equipped with $\mathrm{Ar} / \mathrm{Kr}$ and $\mathrm{He} / \mathrm{Ne}$ lasers and PLAPO 63 oil immersion objective. mRFP was excited with a laser line of excited with a laser line of $\lambda=561 \mathrm{~nm}$ and the fluorescence collected between 555 and $620 \mathrm{~nm}$. GFP was excited with a laser line of $488 \mathrm{~nm}$ and the fluorescence was collected between 493 and $539 \mathrm{~nm}$. DAPI or Calcofluor were excited in the UV $(\lambda=364 \mathrm{~nm})$ and the fluorescence was collected in the range 410 and $470 \mathrm{~nm}$. A focal series of horizontal planes of section were assessed by sequential scanning of sample with $1.0 \mu \mathrm{m}$ step size.

\subsection{DAPI staining of nuclei:}

For the staining with DAPI without fixation $8.3 \mu \mathrm{g} / \mathrm{ml}$ of 4,6-diamidinephenylindole (DAPI) were added to the cells and samples were incubated for 15 $\min$ at RT in the dark before visualization. Staining with DAPI after a short fixation was done as follows: aliquots of cells of about $2 \times 10^{7}$ were collected, sonicated and fixed for $15 \mathrm{~min}$ at $4^{\circ} \mathrm{C}$ in a solution of Formaldheyde
$3.7 \%$ and KPhosphate buffer $0.1 \mathrm{M}$. After centrifugation for $5 \mathrm{~min}$ at 4000 rpm the pellet was washed with PBS and then $5 \mu \mathrm{g} / \mathrm{mL}$ of DAPI was added and incubated for $15 \mathrm{~min}$ at $\mathrm{RT}$ in the dark before observation (Warenda and Konopka 2002).

\subsection{Calcofluor white staining:}

Chitin staining was performed by using $1 \mu \mathrm{g} / \mathrm{ml}$ of Calcofluor White (Sigma, St. Louis, Mo.). For microscopy analyses, cells were stained for $3 \mathrm{~min}$ and washed once with PBS.

In the $\alpha-\mathrm{F}$ experiments in $S$. cerevisiae as well as in other experiments, chitin staining was performed by adding $0.2 \mu \mathrm{g} / \mathrm{ml}$ of Calcofluor White to the culture during the last $10 \mathrm{~min}$ of growth before microscope examination of the cells without fixation (Warenda and Konopka 2002).

\subsection{Rhodamine phalloidin staining:}

Staing with Rhodamine phalloidin (Molecular Probes) in C. albicans was done as follows: $2 \times 10^{7}$ cells were harvested and fixed with formaldehyde (at a final concentration of $3.7 \%$ ) for $2 \mathrm{~h}$ at RT. Cells were centrifuged, washed twice with $1 \mathrm{ml}$ of $\mathrm{dH}_{2} \mathrm{O}$, and resuspended in $500 \mu \mathrm{l}$ of PBS. After that 
cells were sonicated and incubated with $20 \mu \mathrm{l}$ of Rhodamine phalloidin for 45 min in the dark. Finally cells were washed twice with PBS for microscopy visualization.

\subsection{Methylene blue staining:}

Vital dye staining with methylene blue was performed using a standard protocol (Iida, Yagawa et al. 1990). After mild sonication, cells were washed once with $\mathrm{dH}_{2} 0$. Then, an equal volume of a solution of $0.01 \%$ Methylene Blue and $0.2 \%$ Sodium Citrate was prepared and added to the cells. Cells were stained for $10 \mathrm{~min}$ at $\mathrm{RT}$ before counting of positive (dead) and negative (viable) cells under a light microscope.

\section{CALCOFLUOR WHITE SENSITIVITY ASSAY:}

Both in $S$. cerevisiae and C. albicans Calcofluor White sensitivity assay was performed as follows. $5 \mu \mathrm{l}$ from a concentrated suspension of cells corresponding to $\mathrm{OD}_{450 \mathrm{~nm}}$ or $\mathrm{OD}_{600 \mathrm{~nm}}=8$ and $5 \mu \mathrm{l}$ from 1:10 serial dilutions of that suspension, were spotted on SDA or SDA plates in the absence or presence of 2-5-10-20 $\mathrm{mg} / \mathrm{ml}$ of Calcofluor White (Sigma, St. Louis, Mo.). Growth was monitored after 2 days at $30^{\circ} \mathrm{C}$.
7. TREATMENT OF C. albicans CELLS WITH Nocodazole (NZ) and CytochalasinA (CA):

Microtubules polymerization was inhibited by Nocodazole (NZ) (Sigma Aldrich) treatment. Cells were incubated in the presence or absence of NZ for $15 \mathrm{~min}, 1 \mathrm{~h}$ and $2.5 \mathrm{~h}$. The cells were then fixed and stained with DAPI as described previously (Warenda and Konopka 2002) in order to evaluate the efficacy of the inhibitor that should inhibit MTs formation and therefore arrest nuclear division. The quantification of the cells with divided nuclei allowed us to demonstrate that a concentration of $20 \mu \mathrm{g} / \mathrm{ml} \mathrm{NZ}$ was efficient for the inhibition of the microtubule polymerization. The inhibiton of the microtubules polymerization in hyphal cells was done as follows: cells expressing Phr1-GFP protein were split in two cultures and induced to form hyphae in M199 buffered at $\mathrm{pH} 7.5$ (at $37^{\circ} \mathrm{C}$ ) in the absence or presence of $20 \mu \mathrm{g} / \mathrm{ml}$ of nocodazole (NZ) (from a $5 \mathrm{mg} / \mathrm{ml}$ stock in dimethyl sulfoxide [DMSO]) added at the moment of the induction. After $30 \mathrm{~min}, 1.5 \mathrm{~h}$ and $3.5 \mathrm{~h}$ cells were collected, sonicated and stained with DAPI after a short fixation, in order to in order to preserve GFP fluorescence. The 
effects on nuclear division as well as on the green fluorescence were monitored.

To inhibit actin cable formation, cells were treated with Cytochalasin A (CA) from Dreschslera dematoidea (Sigma Aldrich). Overnight cultures of cells expressing the Phr1-GFP fusion protein were diluted to $\mathrm{OD}=0.5$ and induced to form hyphae (in $\mathrm{M} 199 \mathrm{pH} 7.5$ at $37^{\circ} \mathrm{C}$ ) in the presence or absence of various concentrations of CA (stock solution $5 \mathrm{mg} / \mathrm{ml}$ in DMSO). In order to assess for the efficacy of CA as an actin inhibitor we did a Rhodamine-phalloidin staining that allowed us to directly visualize the actin cytoskeleton. Different concentrations $(5,10$ and $20 \mu \mathrm{g} / \mathrm{ml})$ were tested for the efficacy of the inhibitor. Two different experiments with CA were performed: 1) CA was directly added to the culture at the moment in which cells were induced to form hyphae and 2) CA was added after $2 \mathrm{~h}$ after the cultures were induced to form hyphae. By Rhodamine-phalloidine staining we monitored the effect of that the concentrations of $5 \mu \mathrm{g} / \mathrm{ml}, 10$ and 20 $\mu \mathrm{g} / \mathrm{ml}$ on the organization of the actin cytoskeleton. Both concentrations were tested in an experiment in which at $30 \mathrm{~min}, 1 \mathrm{~h}$ and $2.5 \mathrm{~h}$ after the addition of CA aliquots were collected to visualize, after sonication and two washes with
PBS, the green fluorescence under the microscope.

\section{PROTEIN TECHNIQUES:}

\subsection{Total protein extracts from yeast:}

Exponentially growing cells $\left(2 \times 10^{8}\right)$ were collected by filtration, washed with $1 \mathrm{ml}$ of ice-cold water and after 2 mincentrifugation, the pellets were rapidly frozen and stored at $-20^{\circ} \mathrm{C}$. After thawing, $400 \mu \mathrm{l}$ SB-minus buffer $(0.065 \mathrm{M}$ Tris- $\mathrm{HCl} \mathrm{pH} 6.8,5 \%$ SDS, each pellet was supplemented with protease inhibitors $[1 \mathrm{mM}$ phenhylmethylsulfonyl fluoride (PMSF), $1 \mu \mathrm{g} / \mathrm{ml} \mathrm{Pepstanin} \mathrm{A}$ and the Protease Inhibitor Cocktail Complete (Roche)]. After the addition of an equal volume of cold glass beads, cells were broken by shaking in a FastPrep 120 for three cycles of $45 \mathrm{~s}$ at maximum speed alternating with 1 min-incubations on ice. Unbroken cells and glass beads were removed by a 5 min centrifugation at $13.000 \mathrm{rpm}$ at $4^{\circ} \mathrm{C}$. The supernatant was collected and centrifuged again before freezing at $-20^{\circ} \mathrm{C}$. For determination of the protein concentration with the DC Protein Assay (BioRad), aliquots of 20$50 \mu 1$ of the cleared lysates were used in duplicate for each sample. 
8.2. Subcellular fractionation and isolation of the cell walls:

Cells corresponding to $20 \mathrm{OD}_{450}$ for $S$. cerevisiae or $\mathrm{OD}_{600}$ for C. albicans were collected by centrifugation. The pellet was washed with $1 \mathrm{ml}$ ice cold $\mathrm{dH}_{2} \mathrm{O}$ and cells were resuspended in $100 \mu 110 \mathrm{mM}$ Tris- $\mathrm{HCl} \mathrm{pH} 7.5$ supplemented with the proteases inhibitors describe above. Cells were mechanically broken with the presence of glass beads in a FasPrep 120 for three cycles of $45 \mathrm{~s}$ at maximum speed. After removal of the glass beads and centrifugation of the crude extract, the pellet contained the cell walls. The supernatant was centrifuged at $100,000 \mathrm{x}$ $\mathrm{g}$ for $30 \mathrm{~min}$ at $4^{\circ} \mathrm{C}$ and the $\mathrm{S} 100$ and P100 fractions were obtained. The pellet containing the cell walls was processed essentially as described previously (de Groot, de Boer et al. 2004). To remove non covalently linked proteins the pellet was washed with $1 \mathrm{ml}$ of $1 \mathrm{M} \mathrm{NaCl}$ at $4^{\circ} \mathrm{C}$ and then was boiled twice at $100^{\circ} \mathrm{C}$ for 10 min with $1 \mathrm{~m}$ of buffer A (2\% SDS, 100mM EDTA, Tris- $\mathrm{HCl} \mathrm{pH}$ 7,8, 40 $\mathrm{mM} \beta$-mercaptoethanol). After 5 washes with $1 \mathrm{ml}$ of $\mathrm{dH}_{2} \mathrm{O}$ the pellet was resuspended in $30 \mu \mathrm{l}$ of $50 \mathrm{Mm}$ Tris- $\mathrm{HCl}$ pH 7.4 containing 20 U Quantanzyme, a recombinant $\quad \beta-(1,3)$-glucanase (Qbiogen Europe), and protease inhibitors. After $16 \mathrm{~h}$ of digestion at $37^{\circ} \mathrm{C}$ under gentle shaking, the sample was centrifugated at $11,000 \mathrm{x} \mathrm{g}$ for $5 \mathrm{~min}$. The supernatant was denatured in SDSsample buffer. The pellet was boiled in buffer $\mathrm{A}$ for $10 \mathrm{~min}$ to inactivate the enzyme. After 5 washes with $\mathrm{dH}_{2} \mathrm{O}$ the pellet was resuspended in $30 \mathrm{ml}$ of $50 \mathrm{mM}$ phosphate buffer $\mathrm{pH} \quad 6.3$ containing $0.3 \mathrm{mg}$ of pure exo-chitinase of Serratia marcescens (a kind gift of Dr. Enrico Cabib) together with protease inhibitors. After overnight digestion at $37^{\circ} \mathrm{C}$ the sample was centrifugated and the supernatant was denatured for SDSPAGE.

\subsection{DRM isolation:}

Isolation of the detergent resistant membranes (DRMs) was performed as previously described (Bagnat, Keranen et al. 2000). Yeast cells corresponding to $25 \mathrm{OD}_{450 \mathrm{~nm}}$ were mechanically broken in a FastPrep 120 in 500 $\mu$ l of TNE buffer (50mM Tris- $\mathrm{HCl}, \mathrm{pH} 7.4,150 \mathrm{mM} \mathrm{NaCl}$ and 5mM EDTA) supplemented with protease inhibitors as described above. The lysates were cleared by centrifugation at $500 \mathrm{x} \mathrm{g}$ for $5 \mathrm{~min}$ at $4^{\circ} \mathrm{C}$. Two cleared lysates were pooled and incubated with $1 \%$ Triton $\mathrm{X}-100$ for $30 \min$ at $4^{\circ} \mathrm{C}$. After the detergent treatment, about $1.5 \mathrm{ml}$ of lysate was adjusted to $40 \%$ OptiPrep (SigmaAldrich) and $4.2 \mathrm{ml}$ of the resulting 
mixture was sequentially overlaid with $6.7 \mathrm{ml}$ of $30 \%$ OptiPrep in TNEX buffer (TNE buffer including $0.1 \%$ Triton $\mathrm{X}$ 100) supplemented with protease inhibitors and $1.1 \mathrm{ml}$ of TNEX buffer. The samples were centrifuged at 100,000 $\mathrm{x} \mathrm{g}$ in SW41Ti rotor for $2.5 \mathrm{~h}$. At the end, 0.9 ml-fractions were colleted from the top of the gradient except for the first fraction. Proteins were precipitated with $10 \%$ trichloroacetic acid after 1hincubation on ice. Proteins were analyzed by Western blot.

\subsection{SDS-PAGE and immunoblotting:}

For SDS-polyacrylamide gel electrophoresis (PAGE), appropriate amounts of a concentrated solution were added to the lysates in order to bring the samples to a final concentration of $10 \%$ glycerol, 5\% $\beta$-mercaptoethanol, and $0.02 \%$ bromophenol blue. Before loading, samples were denatured at $100^{\circ} \mathrm{C}$ for 3min. SDS-PAGE and immnunoblot were performed as described in (Ragni, Coluccio et al. 2007). Monoclonal mouse anti-actin antibody, clone C4 (MP Biomedicals), was used at a dilution of 1:1000 in Trisbuffered saline (TBS)-5\% bovine serum albumin (BSA), $\quad 0.1 \% \quad$ Tween-20. Monoclonal mouse anti-GFP antibody (Roche) was used at a diluton 1:4000.
Anti-Gas1p serum was obtain by immunizing rabbits with a soluble 6XHis-tagged form of Gas1p produced in Pichia pastoris (Ragni, Fontaine et al. 2007). Immunization procedure was carried out by Areta Intenational S.r.l. (Gerenzano, Varese, Italy). The optimal dilution of anti-Gas1p serum was 1:1000 in TBS-BSA, $0.2 \%$ Tween-20. Peroxidase-conjugated affinity -purified $\mathrm{F}(\mathrm{ab})_{2}$ fragment anti-rabbit or antimouse IgG were from Jackson Laboratories and used at a dilution 1:10000. Bound antibodies were revealed using ECL Western blotting detection reagents (Amersham Pharmacia Biotech). Densitometric measurements of under-saturated films were performed using the Scion Image software.

\section{ADHESION AND INVASION ASSAYS IN Candida albicans}

These experiments were done during my stage in the Fraunhofer Institute [FHG] in Stuttgart, Germany.

\subsection{Cell cultures:}

All cell culture procedures were performed in the cell culture facility of the Fraunhofer Institute. Two human cell 
lines, Caco-2 (ATCC HTB-37), an intestinal epithelial cell line and TR146, a buccal epithelium cell line from the oral cavity, were grown in $75 \mathrm{~cm}^{2}$ tissueculture flasks (Greiner). For both cell lines Dulbecco's Modified Eagle's medium (DMEM) supplemented with $10 \%$ heat-inactivated fetal calf serum (FCS) and $1 \mathrm{mM}$ sodium pyruvate (w/o gentamycin) was used. Cells were cultivated in an incubator at $37^{\circ} \mathrm{C}$ under $5 \% \mathrm{CO}_{2}$. Periodic replacement of the medium and subculture of the proliferating cells were performed until the cells were forming a confluent monolayer.

\subsection{Adhesion assays:}

The adhesion assays were set up in 24-well-polystyrene plates (Greiner). The cavities were either untreated, or pretreated to establish a confluent monolayer of Caco-2 or TR146 cells on the bottom of the well, using D-MEM without gentamycin $+10 \%$ FCS medium. Once the plates were ready for the experiment, the culture medium was removed and $250 \mu \mathrm{l}$ of fresh D-MEM (pre-warmed at $37^{\circ} \mathrm{C}$ ) was added to each well. C. albicans cells were grown overnight at $30^{\circ} \mathrm{C}$ in YPD buffered at $\mathrm{pH}$ 6.0 and then $3 \times 10^{2}$ cells (in $50 \mu \mathrm{l}$ ) were added to each well (for 5 time points in duplicate for each strain).
Plates were incubated at $37^{\circ} \mathrm{C}$ under $5 \%$ $\mathrm{CO}_{2}$. After 30, 60, 120 and $240 \mathrm{~min}$ the medium was removed to plate the nonadherent $C$. albicans cells onto YPD agar plates. The wells were then washed with $300 \mu$ of phosphate-buffered saline (PBS) and after the addition of new PBS C. albicans adherent cells were scratched off and spread onto agar plates. For error documentation adherent and non-adherent $C$. albicans cells were plated immediately after the infection (time point 0). YPD plates were incubated at $30^{\circ} \mathrm{C}$ until next day. Numbers of CFU were then counted and the $\%$ of adherent cells was calculated using statistic parameters.

\subsection{Invasion assays:}

The ability of $C$. albicans strains to invade human tissues was tested using a reconstituted human epithelial (RHE) system of the intestinal cell line Caco-2. The system was developed in the $\mathrm{S}$. Rupp's laboratory and it has been recently described in Methods Mol Biol. (Hernandez and Rupp, 2009). It is composed of a collagen type I matrix overlaid by a monolayer of Caco-2 epithelial cells. Acidic collagen solution (collagen type I from rat tail tendons dissolved in $0.1 \mathrm{M}$ acetic acid) was mixed with an apropriate volume of gelificant solution (2 x DMEM, 0.25 M 
HEPES, $0.8 \% \mathrm{NaHCO}_{3}, 20 \%$ FCS, 0.22

$\mathrm{M} \mathrm{NaOH}$ and $1 \mathrm{x}$ gentamycin) to form solid gel of $\mathrm{pH}$ about 7.4. $300 \mu \mathrm{l}$ of collagen mixture with gelificant solution was let to solidify in 24-well plate inserts (ThinCert, Greiner) for $15 \mathrm{~min}$ at $37^{\circ} \mathrm{C}$ under $5 \% \mathrm{CO}_{2}$. Each insert was transferred to a well of a 24-well plate containing $0.5 \mathrm{ml}$ of supplemented
DMEM and overlaid with $100 \mu \mathrm{l}$ of supplemented DMEM containing $1 \times 10^{5}$ Caco-2 cells. After two days of cultivation the inserts were infected with $10 \mu \mathrm{l}$ of stationary grown Candida cells in YPD-150 mM HEPES pH 6.0 diluted into $\mathrm{dH}_{2} \mathrm{O}$ to $\mathrm{OD}_{600}=0.1$ (about $2.8 \mathrm{x}$ $10^{4}$ cells).

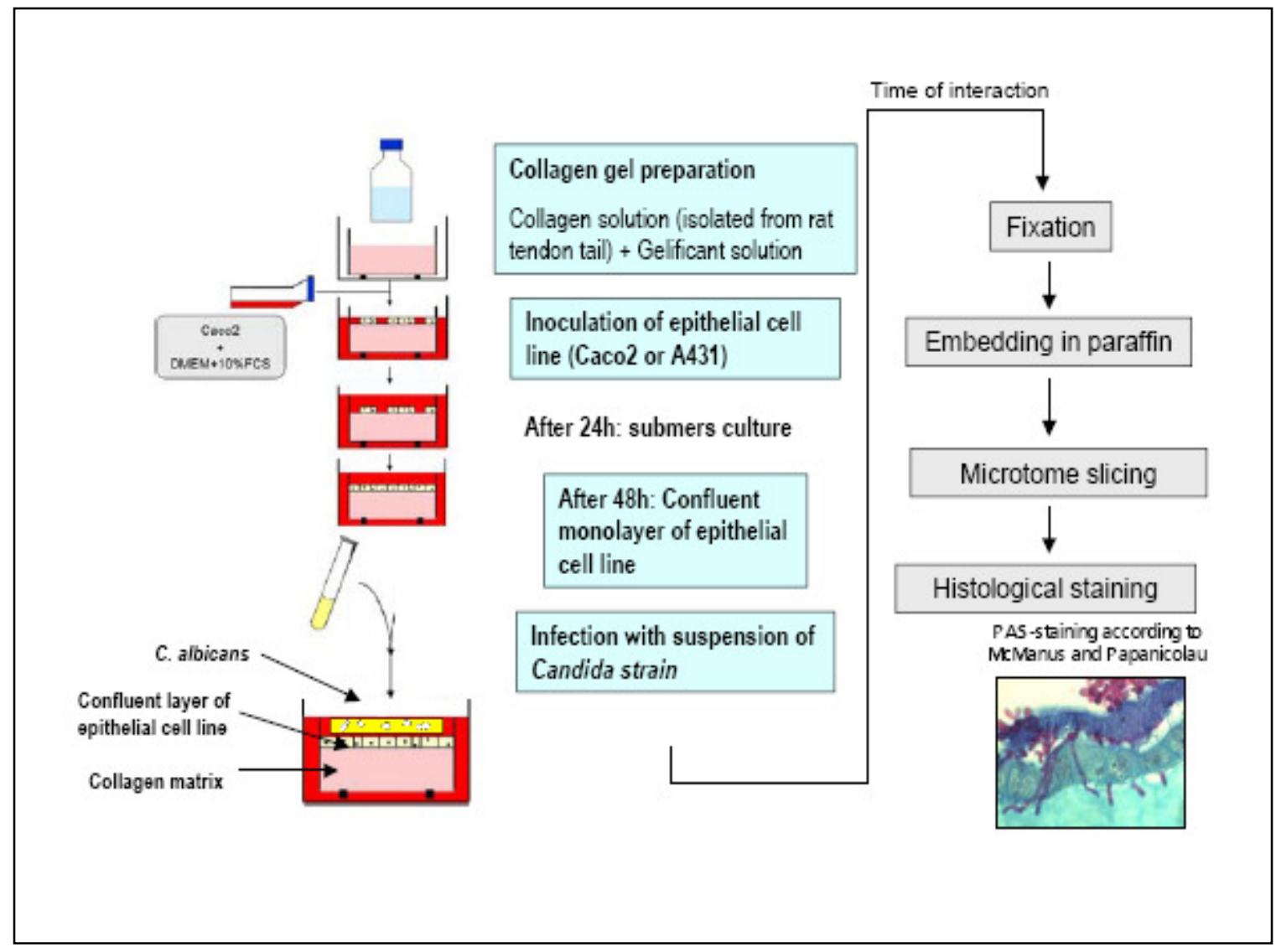

Figure 1. Scheme of the procedure used for the invasion assays

After two days of cultivation the inserts were infected with $10 \mu \mathrm{l}$ of stationary grown Candida cells in YPD$150 \mathrm{mM}$ HEPES pH 6.0 diluted into $\mathrm{dH}_{2} \mathrm{O}$ to $\mathrm{OD}_{600}=0.1$ (about $2.8 \times 10^{4}$ cells). After $7 \mathrm{~h}$ and $24 \mathrm{~h}$ the inserts were fixed for one hour in Bouin's solution (Sigma). The samples were then placed into cassettes to proceed with the embedding process that were 
automatically done into a Shandon

Citadel Tissue Processor. The paraffin blocks were then cut into sections of 5 $\mu \mathrm{m}$ using a microtome (Leica RM2145). Histological staining was done using the PAS method and the method of Papanicolau. After that, the samples were observed under a light microscope (Zeiss, Axiovert 200M).

\section{Quantification of mRNA} using Real Time Quantitative reverse transcriptase $\mathbf{P C R}$

\section{(qRT-PCR):}

RT-PCR experiments were performed in Stuttgart (Germany) in the frame of a collaboration with the laboratory of Dr. Stephen Rupp. Stationary phase cells from an overnight culture in YPD-150 $\mathrm{mM}$ HEPES $\mathrm{pH} 6.0$ at $30^{\circ} \mathrm{C}$, under an agitation speed of $160 \mathrm{rpm}$, were pelleted and suspended in sterile $\mathrm{dH}_{2} \mathrm{O}$ at an $\mathrm{OD}_{600 \mathrm{~nm}}=1.0 .1 \mathrm{ml}$ of this suspension was immediately added to 10 $\mathrm{ml}$ of pre-warmed DMEM medium in a cell culture flask to induce adhesion to the polystyrene surface. The flask was placed in an incubator at $37^{\circ} \mathrm{C}$ under $5 \%$ $\mathrm{CO}_{2}$. Cells from $1 \mathrm{ml}$ of the cell suspension from the YPD culture were collected by centrifugation and quickly frozen in liquid nitrogen. After $2 \mathrm{~h}$ adhesive cells were detached from the plastic surface with a cell scraper and collected together with the non-adhesive cell fraction. Cell pellets from both conditions were frozen in liquid nitrogen as well.

Frozen pellets were mechanically broken mechanically by grinding ina a precooled grinder using a Retsch-mill. RNA was further isolated using RNEasy mini kit (Qiagen). Up to $1 \mathrm{ug}$ of isolated RNA was transcribed into cDNA using QuantiTect Reverse transcription kit (Qiagen). Samples of cDNA were diluted 1:50 and used for qRT-PCR in a LightCycler 480 system (Roche) with universal probe library and respective oligonucleotides (Table 5).

\begin{tabular}{ll} 
Gene & \multicolumn{1}{c}{ Left primer (5'-3') } \\
TDH3 & GCCGTCAACGATCCATTC \\
ALS3 & TGAATATTGGTCTCAATCATTTGC \\
EAP1 & CCACTCCAATTCAACCAACA \\
HWP1 & ATTGCTCCAGGTGCTGAAAC \\
ECE1 & TCAAGCTGCCATCATCCAC
\end{tabular}

Right primer (5'-3') AGAATCGTATTTGAACATGTAAGCA GTGTTTCCTGGTGGTCCAGT TCGTGTAGGAGGTAGATTCAACAA TTCCGGAATAGTAATAGCACCAC GCAGCTGGAGCAACATCTCT

Table 5. Sequences of the oligonucletides used for RT-PCR experiment 


\section{OBJECTIVES OF THIS THESIS:}

The objective of this thesis was a functional characterization of the glycosylphosphatidylinositol-containing proteins Phr1p and its homologous Gas1p in the dimorphic fungal pathogen $C$. albicans and in the budding yeast $S$. cerevisiae. By exploiting the use of fluorescent protein fusion techniques we undertook a cell biology study of the localization of $C a \mathrm{Phr} 1 \mathrm{p}$ and $S c \mathrm{Gas} 1 \mathrm{p}$ in order to elucidate the role of these plasma membrane proteins in different morphogenetic processes. Moreover, our aim was also to study the role of Phrlp, an important virulence factor, in the pathobiology of Candida albicans by analyzing Phr1p contribution to adhesion and invasion, two early processes which promote the establishment of a $C$. albicans infection in the host.

In particular, the aims of the work were:

1. The analysis of the dynamic localization of Phr1p during yeast and hyphal growth in Candida albicans

2. The use of in vitro reconstituted tissue models of infection to test the ability of phrl null mutant of C.albicans to adhere and invade human tissues

3. The analysis of the localization of Gas1p during vegetative growth in $S$. cerevisiae

4. The study of the plasma membrane localization and cell wall anchoring of Gas $1 p$ in S. cerevisiae to understand whether the cell wall is a specific destination of the protein. 
SUBCELLULAR

LOCALIZATION OF Phr1p

DURING YEAST GROWTH

AND DYNAMIC

LOCALIZATION

DURING HYPHAL GROWTH

IN C. albicans

\section{BACKGROUND:}

The cell wall of $C$. albicans is in intimate contact with host cells and is highly dynamic, responding to the environment, growth phase and stresses conditions. C. albicans cell wall is remodelled as the fungus switches morphology or is challenged by agents that compromise its integrity. Alterations in Candida albicans cell surface could influence in the ability of the fungus to evade the host's immune system and to colonize different sites of the body (Nather and Munro 2008). C. albicans is provided with a series of compensatory mechanism to ensure cell wall integrity.

Phr proteins of Candida albicans are members of the Family GH72 of the Glycosylhydrolase classification which are

putative

glycosylphosphatidylinostitol-anchored

cell surface proteins and are endowed

with $\quad \beta$-(1,3)-glucanosyltransferase activity. Mutants lacking these proteins are unable to establish cross-links between $\beta$-(1,6)- and $\beta$-(1,3)- glucans (Fonzi 1999; Ragni, Fontaine et al. 2007). In particular Phrlp, is known to be required for proper morphogenesis and is considered as an important virulence factor in Candida albicans (Ghannoum, Spellberg et al. 1995; Saporito-Irwin, Birse et al. 1995).

PHRl gene is regulated in response to the $\mathrm{pH}$ of the culture. PHRI expression is repressed at $\mathrm{pH}$ values below 5.5 and induced at more alkaline values. Null mutants of PHRl show $\mathrm{pH}$-conditional morpological defects. At alkaline $\mathrm{pH}$ phrls mutant is unable to sustain apical growth of either yeast or hyphal growth forms (Saporito-Irwin, Birse et al. 1995). We have also demonstrated that the mutant is completely unable to form hyphae on solid medium (see Annex). Moreover, it has also been demonstrated that PHRl gene contributes to the virulence of Candida albicans. It is known that phrls mutant is avirulent in mouse models of systemic infections but uncompromised in its ability to cause vaginal infection in rats (Ghannoum, Spellberg et al. 1995; De Bernardis, Muhlschlegel et al. 1998).

In Candida albicans the lack of the polymer activity of Phrlp is influenced by the external $\mathrm{pH}$. At $\mathrm{pH} 8$, and not at $\mathrm{pH}$ 4.5, the alkali-soluble/-insoluble 
glucan ratio of phrls mutant is increased of about $50 \%$ compared with the parental strain (Popolo and Vai 1998). As occurs in gasld mutants of $S$. cerevisiae, phrls mutants shows also an increase in the chitin content (approximately a sixfold increased) in order to counteract the weakening of the cell wall, and are also hypersensitive to calcofluor white (Popolo and Vai 1998; Fonzi 1999).

GPI anchoring is an eukaryotic mechanism for attaching proteins to the cell surface. Proteins destined to be GPIanchored have conserved features, an Nterminal sequence for localization to the endoplasmic reticulum (RE) and a Cterminal signal sequence for the attachment of the GPI anchor. GPI anchor is essential in S. cerevisiae and also in Candida albicans and there are many proteins that are known to be GPImodified. Phr1p has a GPI-anchor signal sequence at the $\mathrm{C}$-terminal end of the protein. Nevertheless, there are some evidences that indicate that $\mathrm{Phr} 1 \mathrm{p}$ is not only a plasma membrane protein but it is also covalently linked to the cell wall. A comprehensive proteomic analysis of the covalently linked CWPs in exponentialphase yeast Candida albicans cells has revealed the presence of Phr1p in the cell wall (de Groot, de Boer et al. 2004).
Still considerable efforts must be done in order to fight against Candida albicans infections. There are several reasons to sustain that $\mathrm{Phr} 1$ protein of Candida albicans could be an important potential molecular target for new antifungal drugs. Among these are: (i) the lack of the protein determines strong reduction of growth in vitro and it is considered to be required for virulence in animal models of infection in vivo, (ii) there is no human ortholog, (iii) no uptake by the cell of an inhibitory molecule would be required as it is an exocellular protein that is anchored to the plasma membrane through a glycolipid (GPI) or cross-linked to the cell wall glucan network.

\section{OBJECTIVES:}

The aims of this work were the study of the localization of Phr1 protein during yeast growth as well as during and hyphal formation as an approach to better understand its role in morphogenesis and pathogenesis. 


\section{RESULTS:}

Construction of a functional Phr1-

\section{GFP fusion protein:}

For the study of the localization of Phr1 protein in $C$. albicans we took advance of fluorescent protein fusion techniques. Since the N-terminal signal peptide is required for the targeting to the ER, and the $\mathrm{C}$ - terminal signal sequence is known to direct the attachment of GPI anchor, and both of them are required for maturation and transport to the cell surface, an internal tagging was necessary. Using a GlobPlot prediction (http://globplot.embl.de/) two disordered regions were selected in order to internally tag Phr1p with the Green Fluorescent Protein (GFP) both at the Nterminal and the C-terminal regions. Figure 1A shows the diagram obtained with GlobPlot in which disorder regions are represented in blue and the globular domains in green.

A

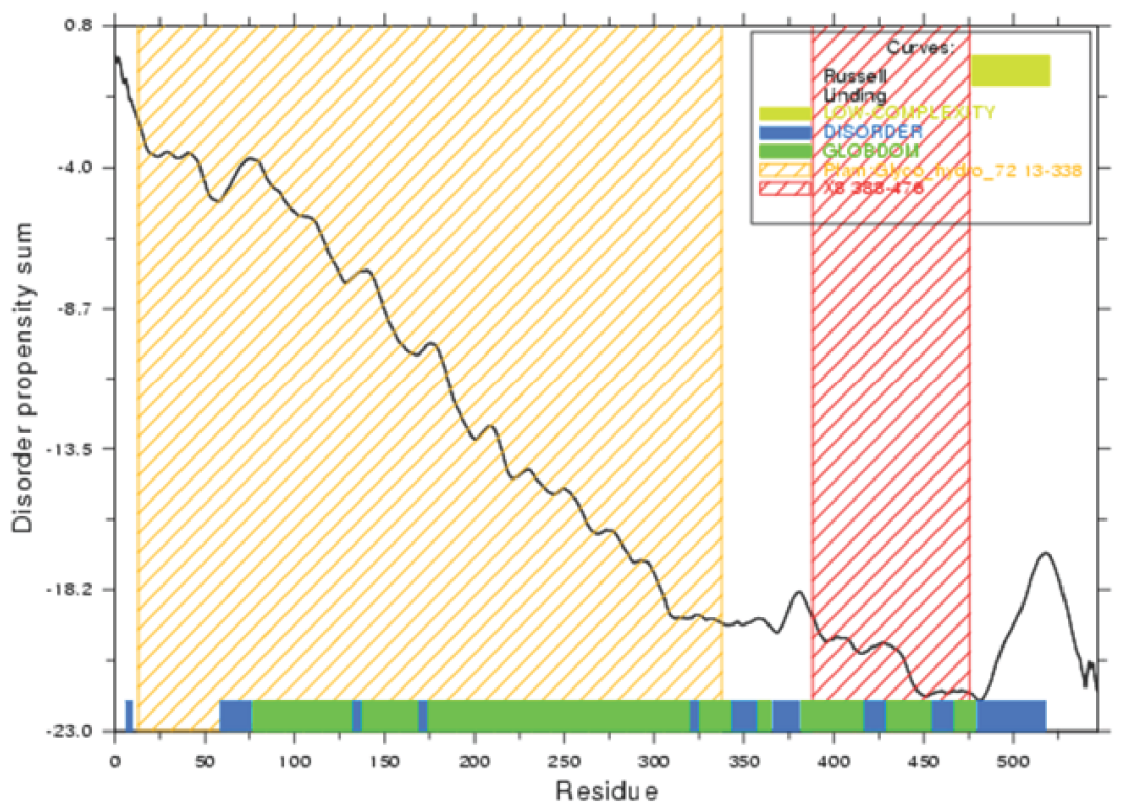

B

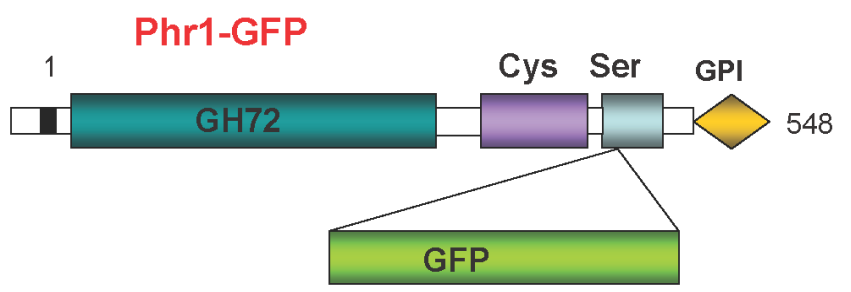

Figure 1. A. GlobPlot (http://globplot.embl.de/) prediction of the disorder regions (blue) and globular domains (green) of Phr1 protein. B. Schematic representation of the tagging of the Green Fluorescent Protein before the GPI-anchor signal of Phr1 protein in Candida albicans. 
For the tagging at the $\mathrm{N}$-terminal region, insertion of GFP was done between S24-S25, whereas for the tagging before the GPI-anchored signal G489-G490 were selected. The strategy used to obtain the transformant strain that expressed the fusion proteins is described in Materials and Methods. Basically, a PCR-based strategy was used. The GFP-URA3-GFP cassette, amplified from pGUG plasmid was used to transformed CAF3-1 strain. Ura+ transformants were checked by PCR for the proper integration of the cassette and plated onto 5-FOA for the excision of the URA3 marker. Transformants obtained in this way were screened first by checking the fluorescence under the microscope by directly picking a colony from the plate and then with a more detail analysis. Preliminary experiment performed with cells growing in the yeast form indicated that fluorescence of the the GFP-Phr1 fusion protein at the $\mathrm{N}$-terminal region gave a weak signal that was not good enough to analyze the localization of the protein (data not shown). Fluorescent emitted by cells expressing the GFP fused to the $\mathrm{C}$ terminal domain of the Phr1 protein was good to perform deep analyses on the localization of the protein. Figure 1B shows the schematic representation of the version of the Phrl protein with the Green Fluorescent Protein fused right before the GPI-anchor signal.

In order to demonstrate that the transformant strain expressed a functional Phr1-GFP tagged protein, Calcofluor White (CW) sensitivity was tested. A representative clone, JC9.4 was plated onto YPD plates without CW (control) or containing 10 or $20 \mu \mathrm{g} / \mathrm{ml}$ Calcofluor White. As shown in Figure 2, the mutant strain, CAS8, grew normally on YPD plates at $\mathrm{pH} 8.0$ without Calcofluor White (CW) whereas on

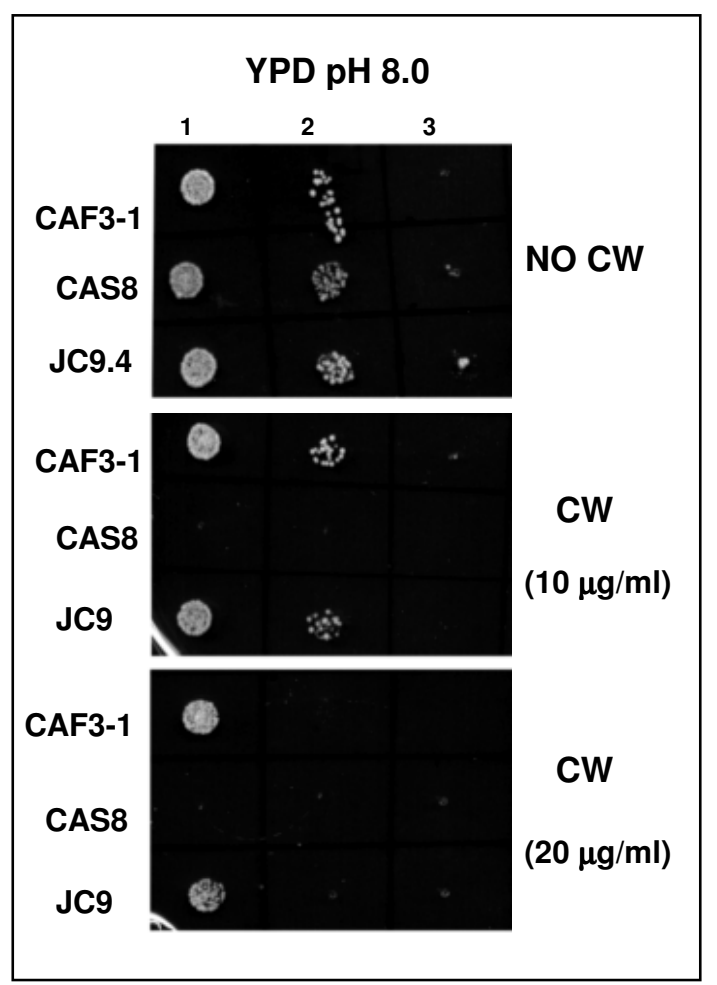

Figure 2. Calcofluor white sensitivity assay of JC9.4 strain expression Phr1-GFP of Candida albicans. 
plates with $10 \mu \mathrm{g} / \mathrm{ml}$ or $20 \mu \mathrm{g} / \mathrm{ml} \mathrm{CW}$, it was unable to grow. The transformant strain JC9.4, that expresses the Phr1GFP fusion protein, did not show the hypersensitivity to calcofluor white characteristic of the mutant strain being able to grow as the parental strain on plates with $10 \mu \mathrm{g} / \mathrm{ml}$ of $\mathrm{CW}$ and only a little bit slower on $20 \mu \mathrm{g} / \mathrm{ml} \mathrm{CW}$ plates.

Western blot analyses of total protein extracts from cells of clone 9.4 were done in both yeast and hyphal growth conditions. For growth in yeast form cells were inoculated in YPD buffered at $\mathrm{pH} 4.5$ or 8.0 and putted in an incubator at $25^{\circ} \mathrm{C}$. Cells were harvested in exponential growing phase and total protein extracts were analyzed by immunoblot. At pH 8.0, a polypeptide of about $118 \mathrm{KDa}$, corresponding with the molecular weight expected for the fusion of the Phr1 protein with the GFP, was detected. As expected for Phr1p, that is expressed at alkaline $\mathrm{pH}$ and repressed at $\mathrm{pH}$ values below 5.5, no signal were detected at $\mathrm{pH} 4.5$ (Figure 3A). Hyphal growth was performed by inoculating cells from an overnight culture in unbuffered YPD at $25^{\circ} \mathrm{C}$ in $\alpha$-MEM medium buffered at $\mathrm{pH} 8$ at $37^{\circ} \mathrm{C}$. For immunoblot analyses cells were harvested at time zero and after $30 \mathrm{~min}$, 1h, 1,5h, 2,5h, 3,5h, 4,5h and 5,5h from the induction. As it shown in Figure 2B, a polypeptide with a molecular weight corresponding to the fusion protein was already detected after 30 min giving a stronger signal from that point forward with the time course of the experiment that reached a pick after 4,5-5,5 h. Actin was used as a reference for the loading.

Taken together, results from these experiments indicate that the internal tagging of Phr1 protein at the C-terminal region expressed a protein that was properly produced and secreted at alkaline $\mathrm{pH}$ in both yeast and hyphal growth. 


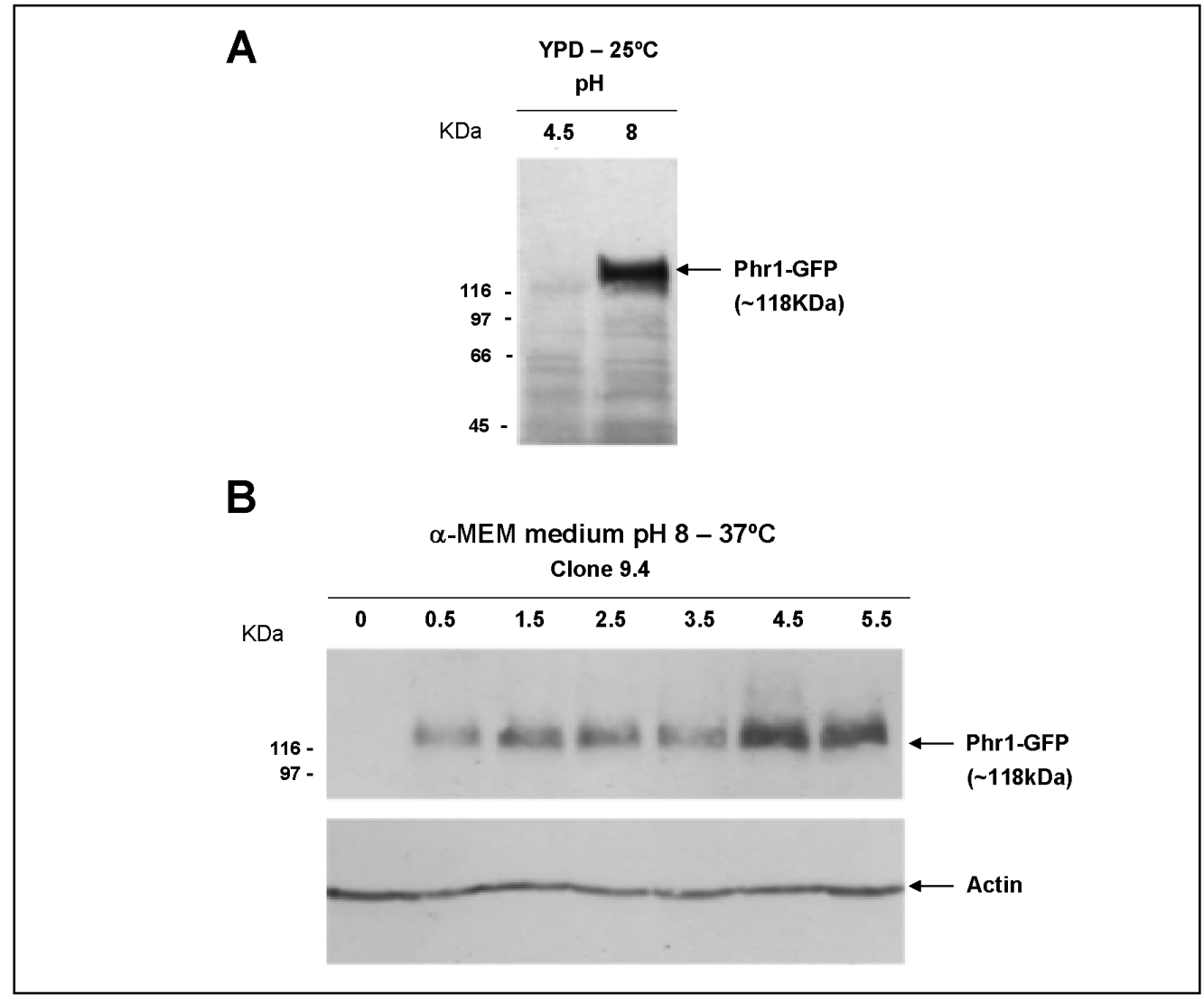

Figure 3. Immunoblot analysis of total protein extracts from A. cells growing in exponential phase in YPD buffered at $\mathrm{pH} 4.5$ or $\mathrm{pH} 8.0$ and $\mathrm{B}$. cells coming from an overnight culture in inbuffered YPD at $25^{\circ} \mathrm{C}$ growing in a-MEM pH 8.0 at $37^{\circ} \mathrm{C}$. Actin served as a control for the loading.

\section{Phr1-GFP protein was found at} discret points on the cell surface and on lipid rafts during vegetative growth:

We used JC9 strain expressing the Phr1-GFP fusion protein to examine the cellular localization of $\mathrm{Phr} 1$ protein in Candida albicans. In order to study the localization of the protein during vegetative growth, cells were incubated in YPD pH 7,5 in batches at $25^{\circ} \mathrm{C}$. After $4 \mathrm{~h}$ cells were harvested, washed twice with PBS and observed under a confocal fluorescence microscope. In a preliminary analysis we observed that Phr1-GFP protein localizes all over the cell surface of exponentially growing cells giving a stronger signal in some specific points. As shown in Figure 4A (left panel), the green fluorescence decorates the cell periphery confirming 


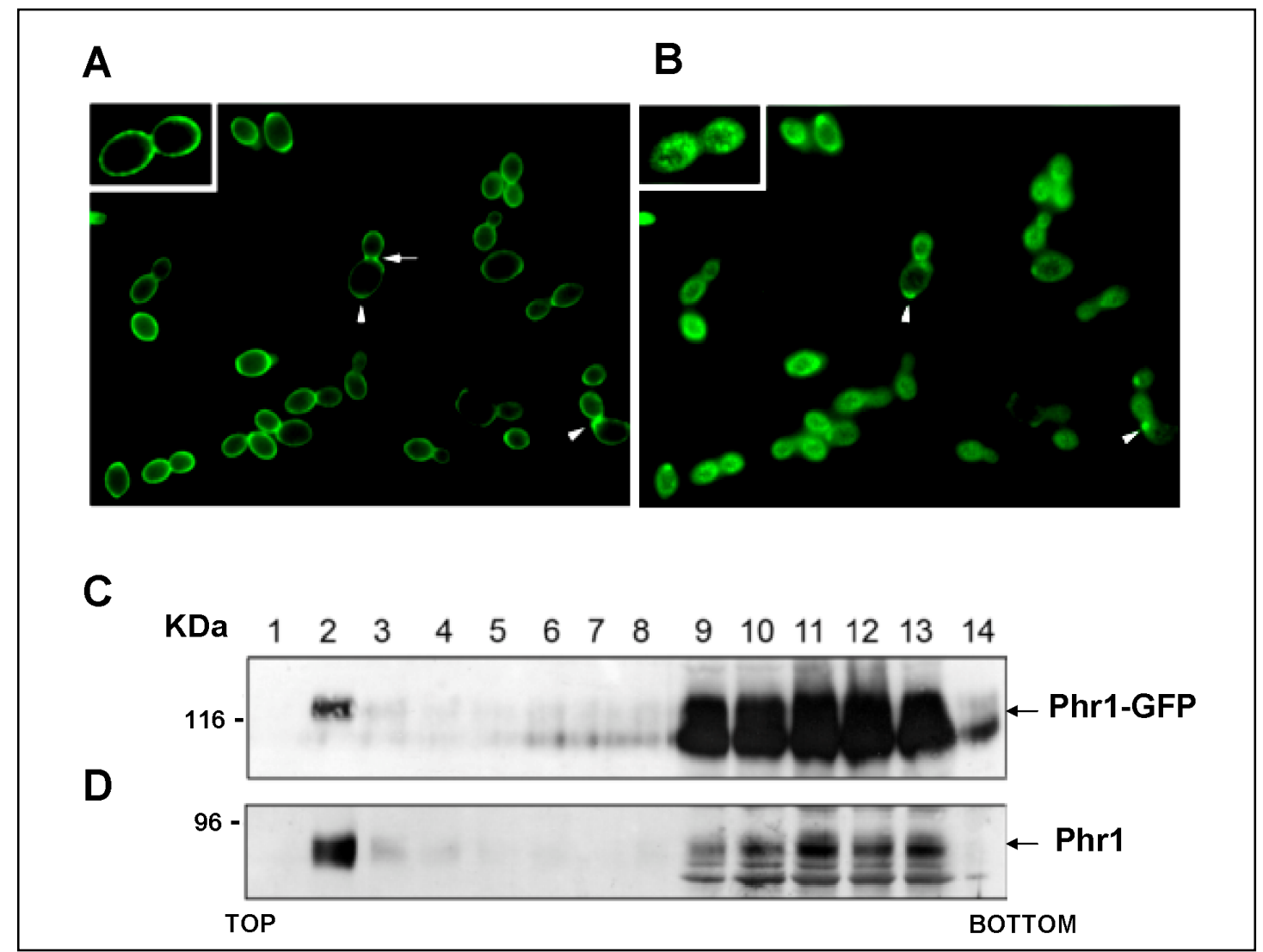

Figure 4. A. Microscopy immage of Candida albicans cells from strain JC9.4 (Phr1-GFP transformant strain) growing in YPD $\mathrm{pH} 8.0$ at $25^{\circ} \mathrm{C}$. B. Image from a different focal plane from the same cells. C and D. Immunoblot of different fractions (from top to botton) of detergent-resistant membranes obtained from JC9.4 cells. Proteins were analyzed by Western Blot using an anti-GFP monoclonal antibody $(\mathrm{C})$ or an anti-Gas1p polyclonal serum $(\mathrm{E})$.

that the protein was properly produced and secreted. We found a stronger signal in the neck region between the mother and daughter cells (see white arrows in Figure 4). In some areas we observed some crater-like structures suggesting that the protein could also localize in the bud scars. Interestingly, we also realized that the green fluorescence over the cell periphery presented a punctuate pattern. These very fine patches or dots are visible in the magnification that is shown in the upper part of the left panel in Figure 4A. Moreover, when we observed single-plane images in the confocal microscope, fine dots all over the cell and in discrete sites around the cell were clearly visible. Gas $1 p$ and Phr1p in Saccharomyces cerevisiae and Candida albicans have been found in several biochemical studies in the DRMs (Bagnat, Keranen et al. 2000; Bagnat and Simons 2002; Insenser, Nombela et al. 2006) and in our laboratory we have shown evidences for the localization of a functional version of Gas 1p-GFP in lipid rafts in Saccharomyces cerevisiae (see Chapter I). To verify that Phr1-GFPp is 
also present in detergent-resistance membranes in C. albicans, Triton X-100 treated extracts from cells expressing the Phr1-GFP protein and from the parental strain were prepared. After centrifugation and fractionation in iodixanol (OptiPrep) discontinuous step gradients, the detergent resistance fraction protein was analyzed by Western Blot. Using an anti-GFP monoclonal antibody, Phr1-GFP was detected at the top of the gradient sharing the same behaviour of Phrlp (Figure $4 \mathrm{C}$ and $\mathrm{D}$ ). The molecular weight of the band was compatible with that expected for the fusion of Phr1 (about 88KDa) and GFP (about 30 KDa). In fractions 6-14 another band of lower size was also detected that could be the result of the accumulation of partially folded forms.

Phr1p is localized at the cell periphery, in the neck region, in the septum and in bud scars during vegetative growth:

To further investigate the localization of Phr1-GFP protein during vegetative growth in Candida albicans, we performed more detailed analysis using confocal microscopy. For this purpose JC9.4 strain expressing the Phr1-GFP protein was grown under conditions that unable budding formation. As reported in Materials and Methods, cells from stationary phase were incubated into YPD buffered at $\mathrm{pH} 7.5$ with $150 \mathrm{mM}$ Hepes at $25^{\circ} \mathrm{C}$. Exponentially growing cells were harvested and processed for fluorescent microscopy analysis. Green fluorescent signal from Phr1-GFP was found at different locations. It decorated the cell periphery consistently with the plasma membrane localization of the protein (Figure 5A; upper panel, right). We also noticed that the signal was brighter in some regions. As shown in the magnification in the upper panel in Figure 5A, the signal was brighter the in emerging buds and in the neck region. Chitin staining with Calcofluor White (blue) was used to visualize the sites in which green fluorescence from Phr1GFP colocalized with the chitin. Light blue color in the merge in Figure 5A represents the overlapping between Phr1-GFP and chitin. Figure 5B shows the overlapping of the fluorescence signals in more detail. As shown in the upper panel of Figure 5B, in medium budded cells the green fluorescent was preferentially located in the emerging bud. Interestingly, two brighter dots (arrow head) were placed at both sides of the mother-daughter neck region. 


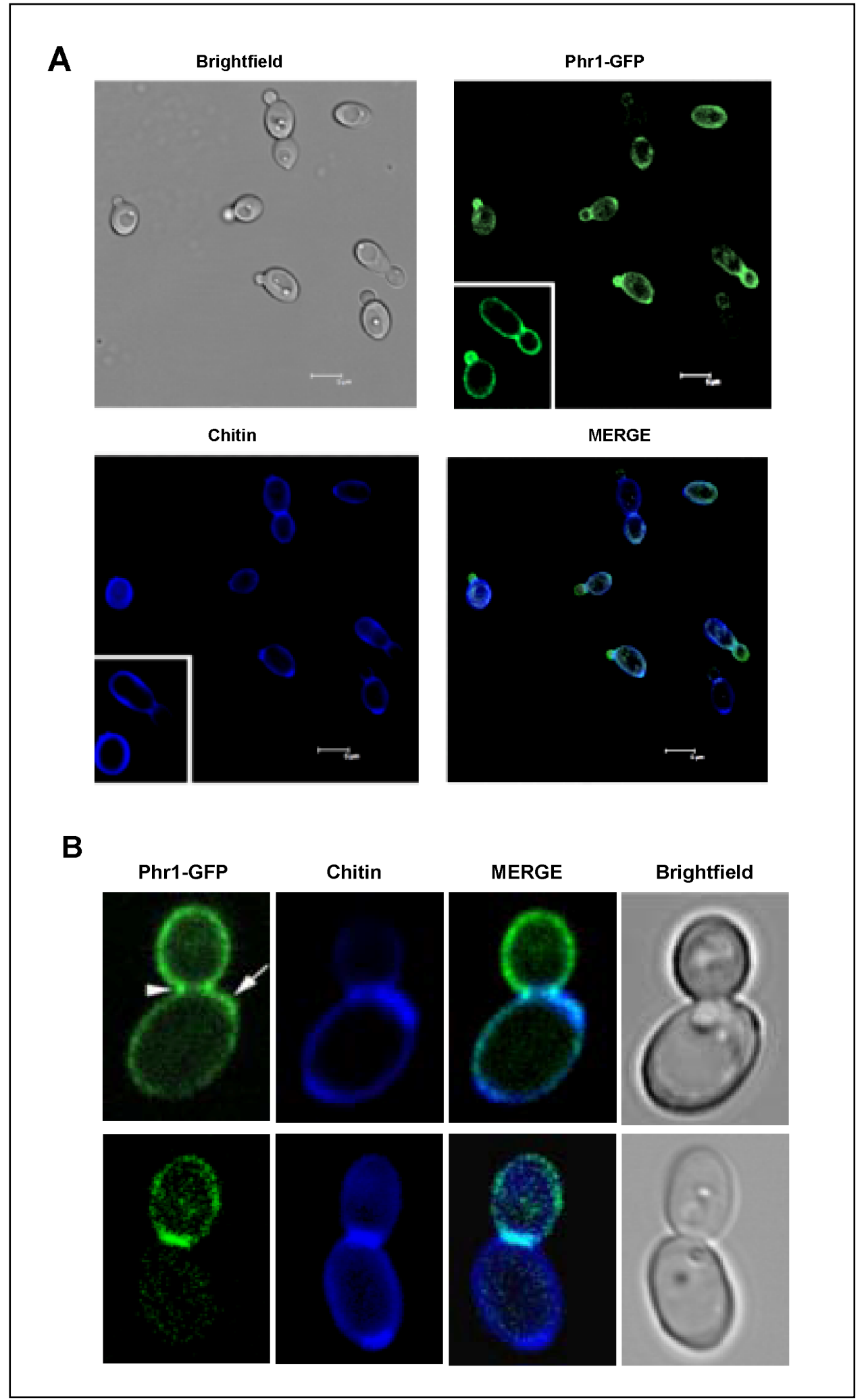

Figure 5. Confocal micrographs of Candida albicans cells expressing Phr1-GFP protein during vegetative growth. A. Cells were grown in YPD buffered at $\mathrm{pH} 7.5$ at $25^{\circ} \mathrm{C}$ and collected during log phase for microscopy analyses. Chitin staining was performed using $1 \mu \mathrm{g} / \mathrm{ml}$ of Calcofluor White. B. Detail of the neck regions in a medium-budded cell (upper panels) and in a large-budded cell (bottom panels). 


\section{CHAPTER I}

In many cells a crater-like structure was also present beside the neck region (arrow in Figure 5B). Chitin signal was stronger in the mother cell and, as expected, it was concentrated in the chitin ring and in bud scars. As shown in the merge in Figure 5B, Phr1-GFP colocalized with the chitin ring and was also present in the bud scar where it also colocalized with the chitin. In largebudded cells (Figure 5B, bottom panel), Phr1-GFP nicely decorated the septum region. Green fluorescent was also found in a segment that appeared to be coincident with the primary septum (light blue color in the bottom panel in Figure 5B).

\section{Loss of PHRI affects the width of the neck region:}

In order to confirm that Phrlp plays an important role in the neck region, we measured the width of the motherdaughter neck diameter in wild type (CAI10) and phrls null mutant cells (CAS10). Measurements of the neck were performed using a confocal microscopy.

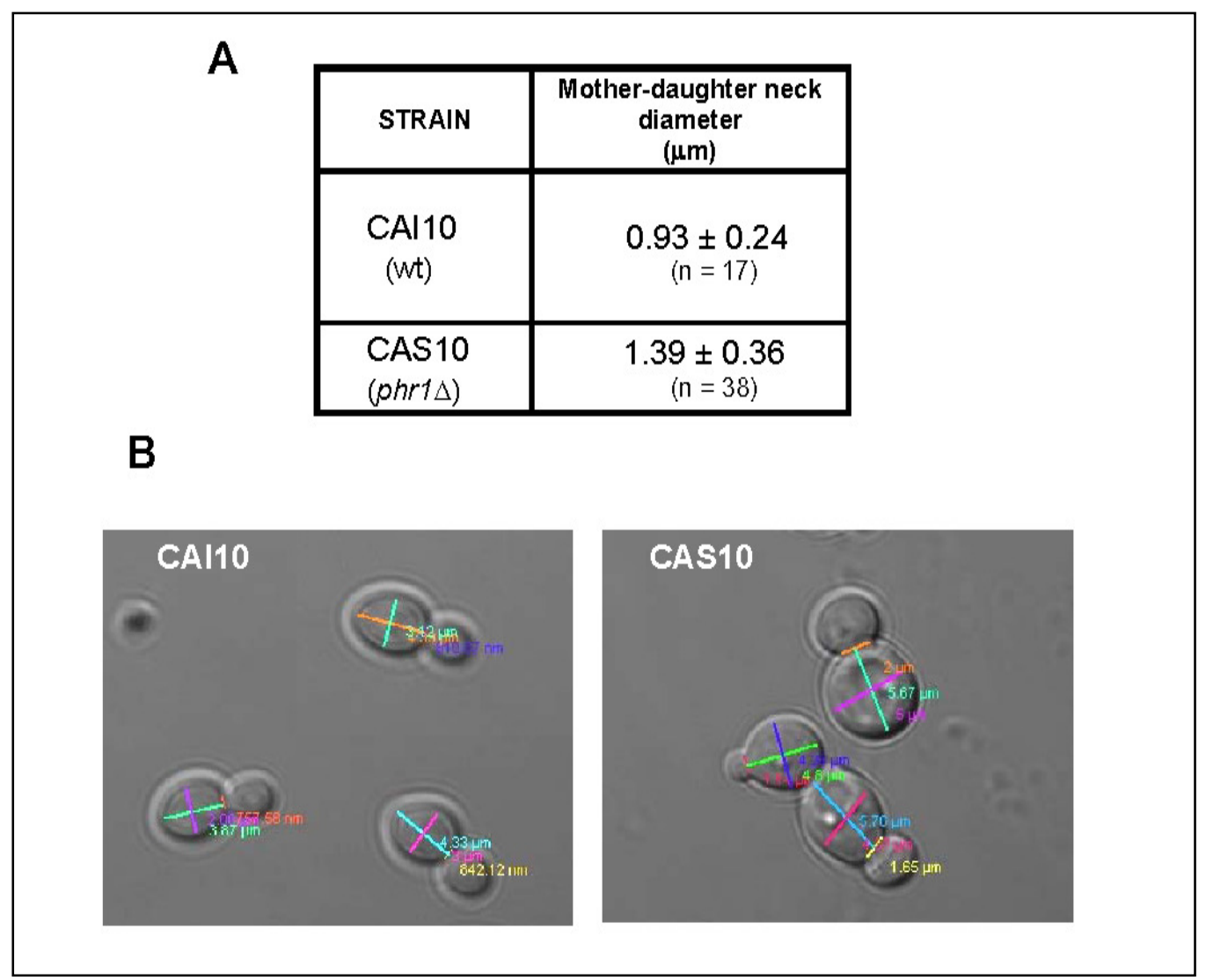

Figure 6. Enlargement of the mother-daughter neck in phrls mutant cells. A. mean values $( \pm$ s.d.) of the measurement of the diameter $(\mu \mathrm{m})$ of the neck region. B. Examples of the micrographs obtained at the confocal microscope where the measurements of the wideness of the neck where performed. 
Since we had found that Phr1-GFP protein is present in the chitin ring, we hypothesized that the localization of the protein in that area might have a biological meaning. If that statement is true, cells lacking Phrlp should have a morphological defect due to the absent of the activity of the protein. As shown in Figure 6, the diameter of the neck is increased by about 1.5 times in the mutant compared with the wild type. Thus, Phrlp might have a role in reinforcing the cell wall in that area that needs to remain constricted during the septation process.

\section{Shitf down from pH 7.5 to pH 4.5:}

The work presented here about the localization of Phr1p in C. albicans was performed in paralleled with the work with Gas1p in S. cerevisiae. While we were analyzing the localization of Phrlp in C. albicans we had already observed that in Saccharomyces cerevisiae a fluorescent version of Gas1p (mRFPGas1p) is present in two fractions: a mobile fraction localized in the plasma membrane and an immobile one which is likely to be bound to the cell wall (see Chapter I), we wanted to investigate whether Phrlp of Candida albicans could present the same behaviour. For this purpose we set up an experiment in which cells growing at $25^{\circ} \mathrm{C}$ in YPD buffered at $\mathrm{pH} 7.5$ were shifted to YPD buffered at $\mathrm{pH}$ 4.5. So with this experiment cells were basically shifted from a $\mathrm{pH}$ in which the protein is expressed at high levels, a $\mathrm{pH}$ in which the protein is not expressed, $\mathrm{pH} 4.5$.

Analysis of the total protein extracts that were took after different time intervals from the down shift of $\mathrm{pH}$ revealed that the levels of the Phr1-GFP protein rapidly decreased when cells were shift to a restrictive $\mathrm{pH}$. As shown in Figures 7A and B, after $30 \mathrm{~min}$ from the shift the relative protein level of the protein was already decreased to a $70 \%$ and after $1 \mathrm{~h}$ the relative protein level was almost $40 \%$. Afterwards the level of the protein continuous decreasing with values below $20 \%$ after $3 \mathrm{~h}$ and being almost undetectable after $7 \mathrm{~h}$. 


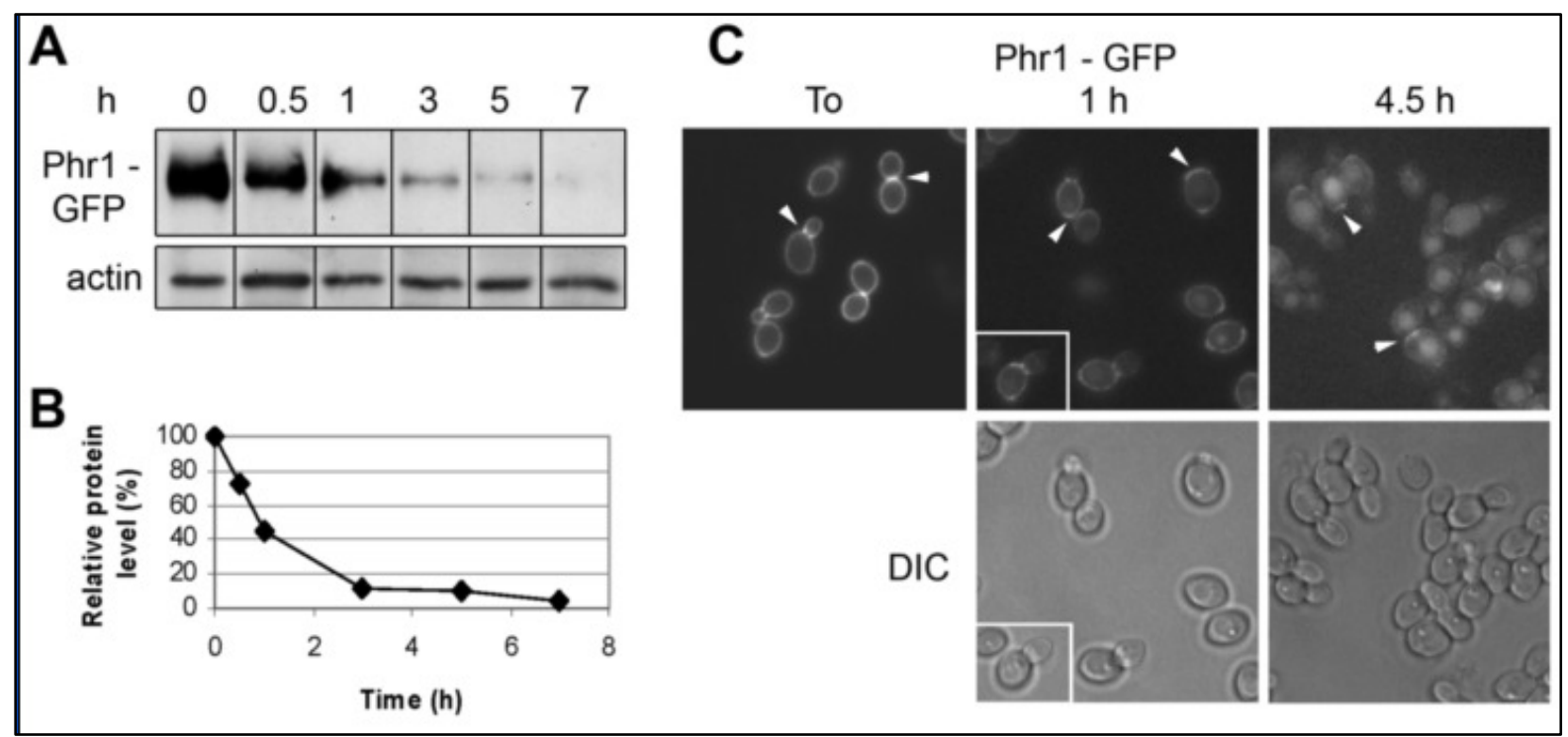

Figure 7: Shift down of $\mathrm{pH}$ in C. albicans cells expressing the Phr1-GFP protein. Cells growing in YPD buffered at $\mathrm{pH} 7.5$ were shifted to YPD buffered at $\mathrm{pH}$ 4.5. A. Western Blot analysis of total protein extracts harvested at different time points from the shift down of $\mathrm{pH}$. B. Relative protein levels of Phr1-GFP that were calculated by normalization with the level of the actin. C. Microscopy analysis of the cells expressing the Phr1-GFP protein during the experiments.

Mycroscopy analysis of the samples harvested at this different time point revealed that the green fluorenscence rapidly diminished. After $1 \mathrm{~h}$ the fluorescence in the contour of the cells almost disappeared been completely undetectable after 4.5h (Figure 7C). Nevertheless the signal was still bright in some specific points and in particular in some crater-like structures that are thought to correspond to the bud scars (head arrows in Figure 7C).

Together these observations point out that the rapid decrease of the level of the protein is due to the absent of de novo synthesis at restrictive $\mathrm{pH}$ but also to a high turnover of the protein that is rapidly removed from the plasma membrane. Strikingly, as occurs in Saccharomyces cerevisieae, the protein present in the bud scars seems to be protected from degradation.

\section{Phr1 protein localizes in the tips of the germ tubes and redistributes during \\ hyphal growth:}

In order to analyze the localization of Phr1p during hyphal growth and in an attempt to gain insights into the role of the protein during filamentation, cells expressing the Phr1-GFP protein were induced to form hyphae in Medium 199 at pH 7.5 and $37^{\circ} \mathrm{C}$. Cells were harvested at time zero and at different time intervals during hyphal formation and 
treated for fluorescent microscopy analysis. At time zero, Phr1-GFP protein was hardly detected as expected from cells coming from a stationary phase culture in unbuffered YPD at $25^{\circ} \mathrm{C}$ in which the protein is not expressed because of the acidic $\mathrm{pH}$. As shown in the left panel in Figure 8, after $30 \mathrm{~min}$ from the induction of the filamentation process Phr1-GFP protein was already detected being concentrated in the tips of the emerging germ tubes, whereas no signal was detected in the cell body. After 1h, when the germ tubes were longer, the green fluorescence was still strong in the tips and it was also present along the lateral walls of the germ tubes (second panel in Figure 8). In the third panel of Figure 8 the hyphae at longer times are shown. When the hyphal growth continues, the signal in the tip is not so strong and Phr1-GFP protein is present all over the hyphal surface. At $3 \mathrm{~h}$ we also observed that the green fluorescence was bright in the septa of the hyphae. After 5.5h Phr1-GFP protein distributed along the hyphal wall and gave a stronger signal in the septa. At this time point the signal in the tips of the hyphae was less strong than at the beginning of the process (see right panel on Figure 8). These observations indicate a polarized localization of $\mathrm{Phr} 1$ protein during germ tube formation and suggest a redistribution of the protein during hyphal growth. Moreover these results reveal an unexpected localization of the protein in the septa of the hyphae.

We further analyzed the localization of Phrlp during hyphal growth using confocal microscopy. The experiments were performed in the same way as described above and, in order to see whether the protein colocalizes with the chitin, a calcofluor white staining was performed as described in Materials and Methods. As shown in Figure 9A, when the primary septum was already formed Phr1-GFP localized in the tip of the hyphae and distributed along the surface of the hyphae. It produced a strong signal in the septum, indicating that the protein concentrated in this site, whereas no signal was detected in the cell body. Chitin is present in the cell body but not in the apical region which allows the hyphae to elongate. As shown in the merge image in Figure 9A, Phr1-GFP colocalized with the chitin in the septum giving an intense light blue color. In longer hyphae the distribution of the fluorescence is similar (Figure 9B). The green fluorescent is primarily localized in the apical region and from that point the intensity of the signal decreased along the surface of the hyphae being coincident with the chitin in the septa. 


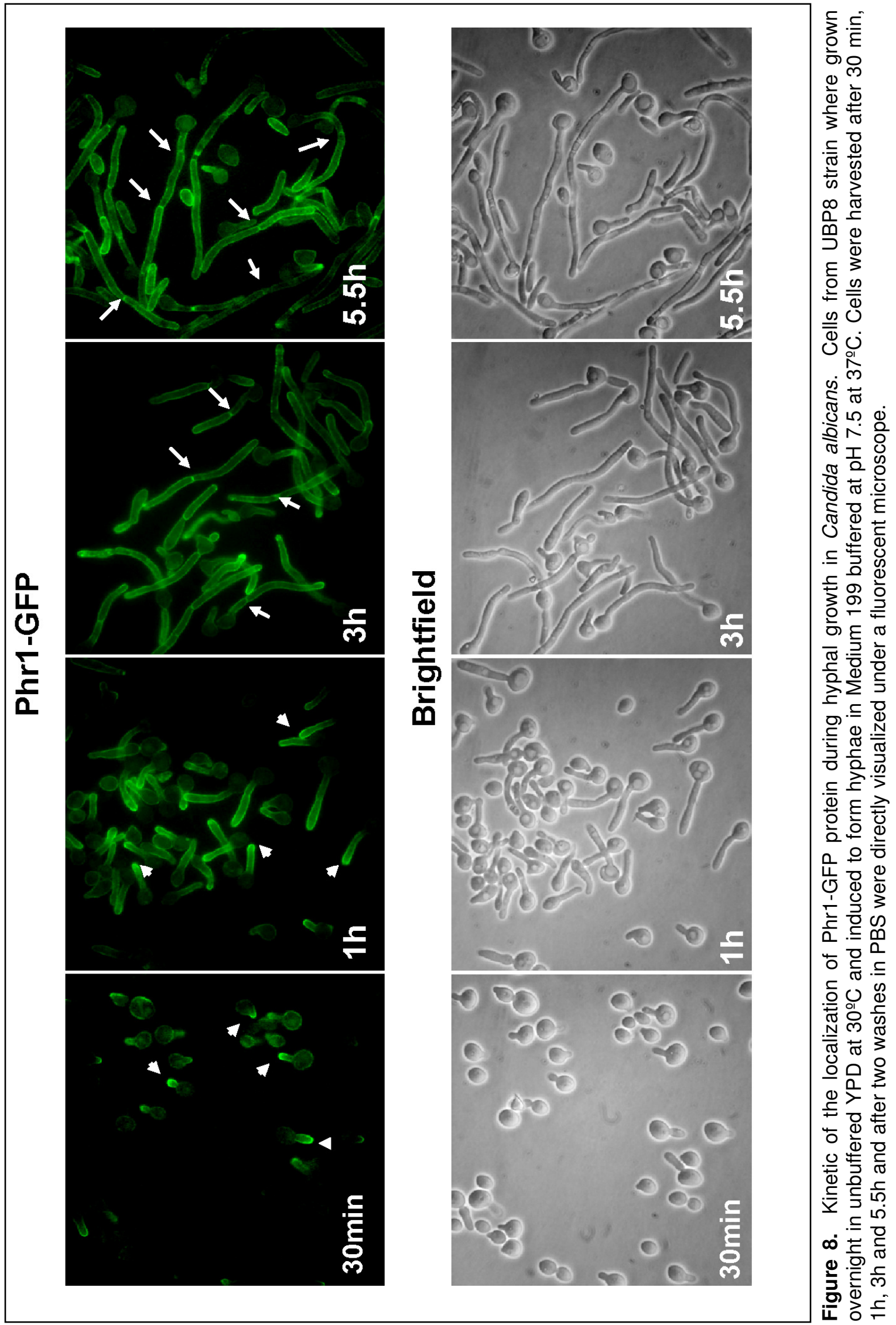




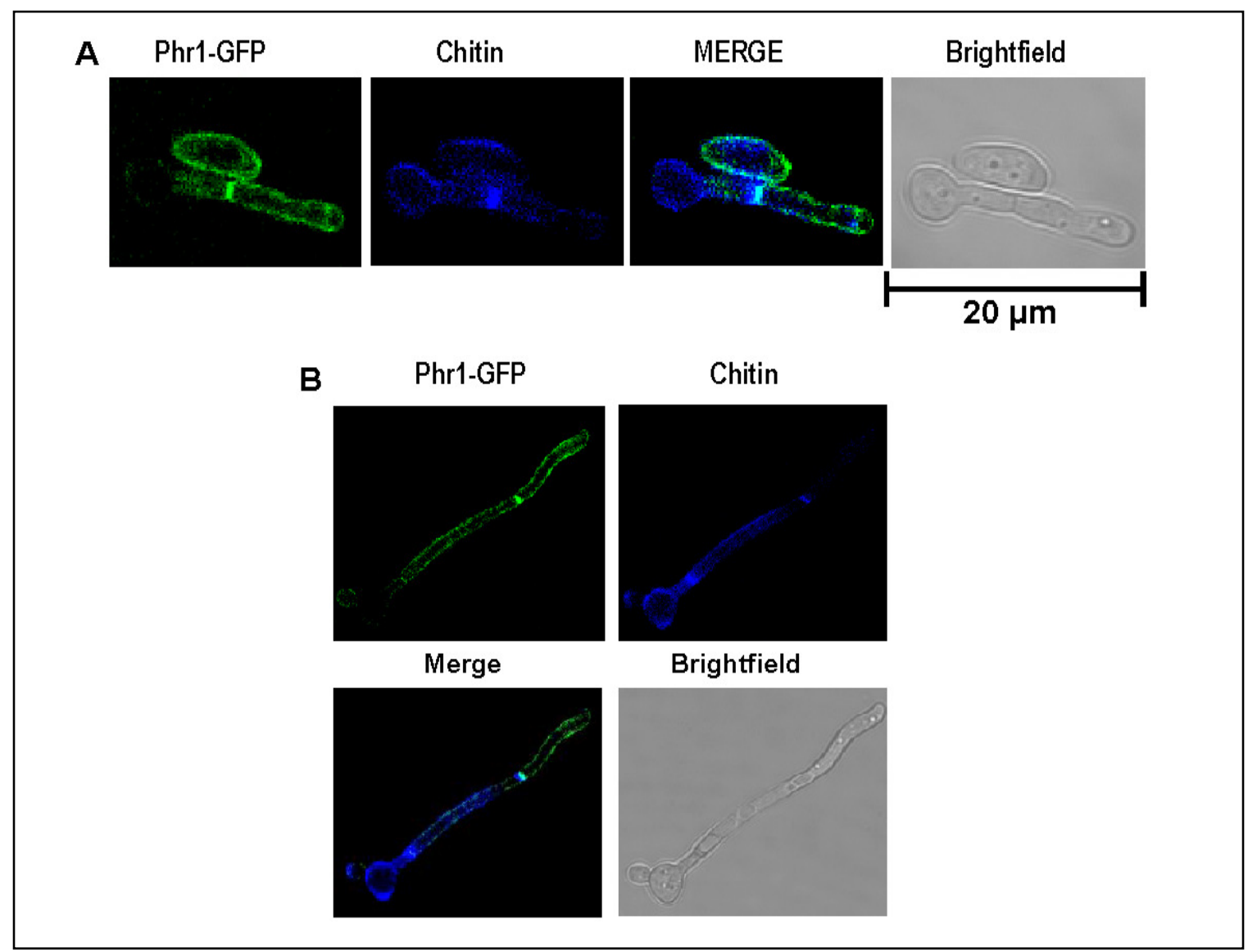

Figure 9. Details of the colocalization with chitin of Phr1-GFP protein in the hyphae. Induction of hyphal formation was performed in Medium 199 at $\mathrm{pH} 7.5$ at $37^{\circ} \mathrm{C}$. Chitin was stained with $0.2 \mu \mathrm{g} / \mathrm{ml} \mathrm{of}$ Calcofluor White that was added directly to the medium during the last 10 minutes of growth. Cells were collected after $1.5 \mathrm{~h}(\mathrm{~A})$ and $3 \mathrm{~h}(\mathrm{~B})$ from the induction. Images were taken under a confocal microscopy.

\section{Polarized localization of Phr1-GPF during hyphal growth does not depend on microtubes:}

To explore the mechanism by which Phr1-GFP localizes in a polarized manner during hyphal formation, we wanted to determine whether the localization of the protein depends on cytoskeletal components. The requirement of microtubules was tested by treating cells with Nocodazole (NZ) which causes microtubule depolymerization. The efficacy of inhibition was assayed quantitatively by monitoring the inhibition of nuclear division both during yeast or hyphal formation (Table 1 and Table 2). DAPI staining of DNA was used to count cells with divided nuclei. Treatment with $20 \mu \mathrm{g} / \mathrm{ml} \mathrm{NZ}$ resulted in a reduction in the number of cells within the population that successfully divided their nuclei (Tables 1 and 2). Moreover, during yeast growth most of the cells treated with NZ arrested as large budded cells (96\% versus $47.2 \%$ in the untreated control) (Table 1). 


\begin{tabular}{ccc}
\hline & $\begin{array}{l}\% \text { cells with } \\
\text { divided nuclei } \\
\text { Cells }\end{array}$ & $\begin{array}{c}\% \text { Large-budded } \\
\text { cells }\end{array}$ \\
\hline$(-) \mathrm{NZ}$ & $25,4 \%(\mathrm{n}=210)$ & $96 \%(\mathrm{n}=232$ \\
\hline$(+) \mathrm{NZ}$ & $7,2 \%(\mathrm{n}=213)$ & $47.2 \%(\mathrm{n}=229)$ \\
\hline
\end{tabular}

Table 1. Cells from strain UBP8 expressing Phr1GFP protein were grown overnight in YPD to stationary phase, diluted to $\mathrm{OD}=0.2$ and let grow in the presence or absence of $20 \mu \mathrm{g} / \mathrm{ml}$ of NZ for 3.5h. Cells were washed once with PBS and kept on ice for $15 \mathrm{~min}$. Status of nuclear division was assessed by staining nuclear DNA with DAPI and the number of cells with divided nuclei was determined directly by counting at least 200 cells. Large-budded cells were those cells where the daughter cell was at least two-third the size of the mother cell.

Together these results demonstrate that this concentration of NZ efficiently inhibited microtubule function and therefore arrested nuclear division in these cells. Importantly, inhibition of microtubule polimerization with $\mathrm{NZ}$ did not affect either the abilitiy of the cells to form hyphae or the rate of formation (data not shown).

\begin{tabular}{cc}
\hline Cells & $\begin{array}{c}\% \text { cells with divided nuclei } \\
\text { (hyphal growth) }\end{array}$ \\
\hline$(-) \mathrm{NZ}$ & $35,1 \%(\mathrm{n}=217)$ \\
\hline (+) NZ & $6,7 \%(\mathrm{n}=221)$ \\
\hline
\end{tabular}

Table 2. Cells from strain UBP8 expressing Phr1GFP protein were grown overnight in YPD to stationary phase, diluted to $\mathrm{OD}=0.2$ and induced to form hyphae in the presence of absence of $20 \mu \mathrm{g} / \mathrm{ml}$ of NZ for $1.5 \mathrm{~h}$ before harvesting. DAPI staining allowed us to quantify the number of cells with divided nuclei by counting at least 200 cells.

In order to determine whether the localization of Phr1 protein to the tips of the germ tubes depends on microtubules, we performed an experiment with cells expressing the Phr1 GFP fusion protein starting from stationary phase cells that were diluited to $\mathrm{OD}=0.5$. Cells were induced to form hyphae at $37^{\circ} \mathrm{C}$ in Medium 199 buffered at $\mathrm{pH} \quad 7.5$ containing $20 \mu \mathrm{g} / \mathrm{ml}$ of NZ. To verify that microtubules were depolymerized, nuclei were stained with DAPI as described in Materials and Methods. Quantification of divided nuclei demonstrated that in most of the cells treated with NZ nuclear division was arrested (Figure 10A, left panels).

As shown in Figure 10A (right panels) Phr1-GFP was highly polarized, decorating with a bright signal the tips of the germ tubes, independently of the addition of NZ to the culture (Figure 10B). Thus, when germ tubes were emerging microtubule disfunction did not affect the localization of Phr1-GFP. 


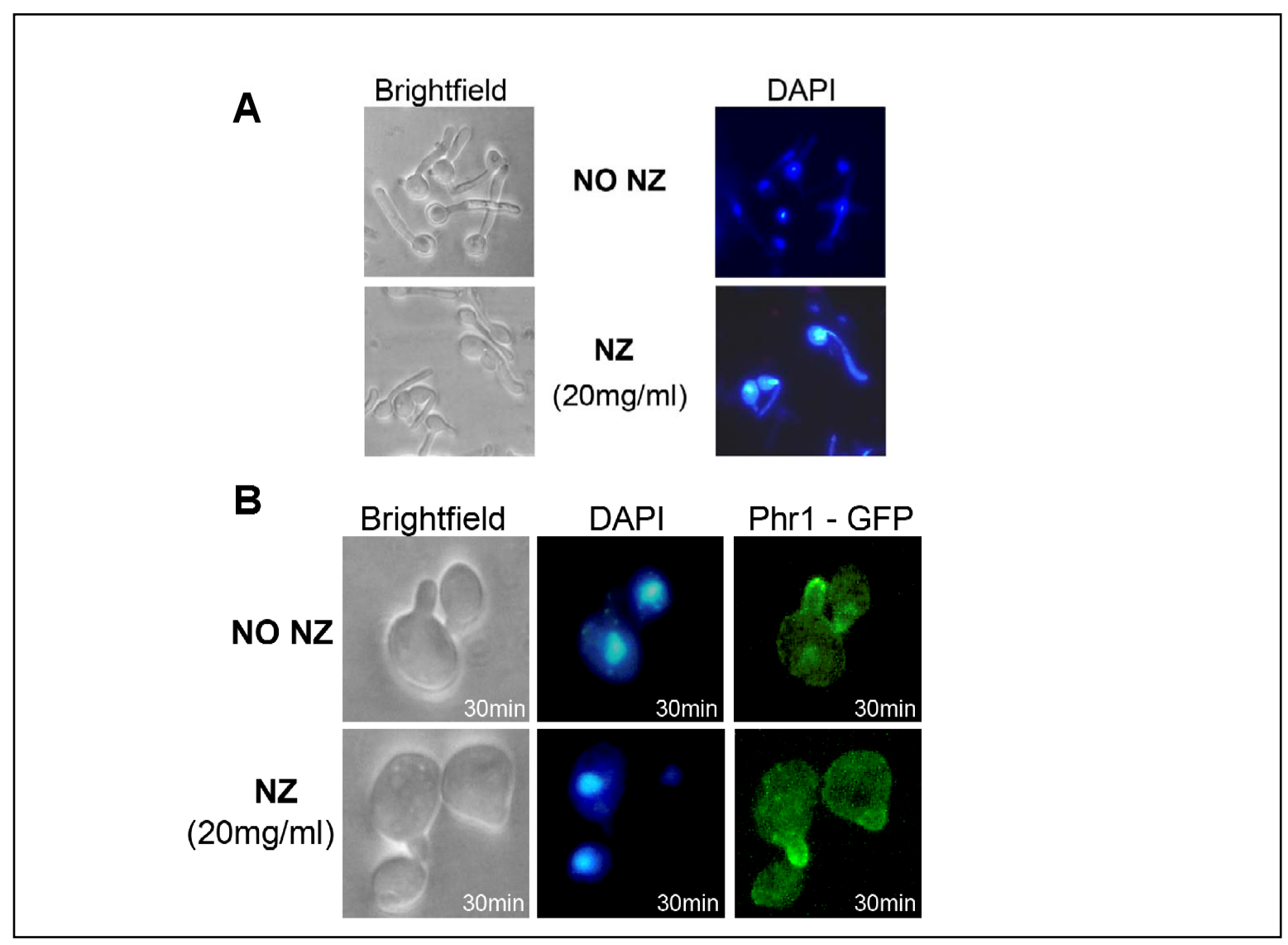

Figure 10. Effect of Nocodazole on germ tube formation in Candida albicans. Cells (UBP8 strain) were induced to form hyphae in Medium 199 at $\mathrm{pH} 7.5$ at $37^{\circ} \mathrm{C}$ with or without Nocodazole (NZ) that was added directly to the culture in the moment of the hyphal induction. (A) $20 \mu \mathrm{g} / \mathrm{ml}$ of NZ were sufficient to arrest nuclear division and therefore inhibit microtubule polarization. (B) Germ tubes formed after 30 minutes from the induction of the filamentation in untreated cells (upper panels) or in cells treated with $20 \mu \mathrm{g} / \mathrm{ml}$ of $\mathrm{NZ}$.

Afterwards, the germ tubes continued growing and hyphae formation occurred normally. In cells treated with NZ Phr1GFP was found to localize in the distal region and in the lateral walls of the hyphae as in the control cells. Strikingly, by making a calcofluor white staining we observed that cells treated with NZ presented an abnormal chitin deposition in the septa.

As shown in Figure 11, in cells treated with NZ many septa were thicker and in others chitin was deposited abnormally producing septa that were not totally closed. 


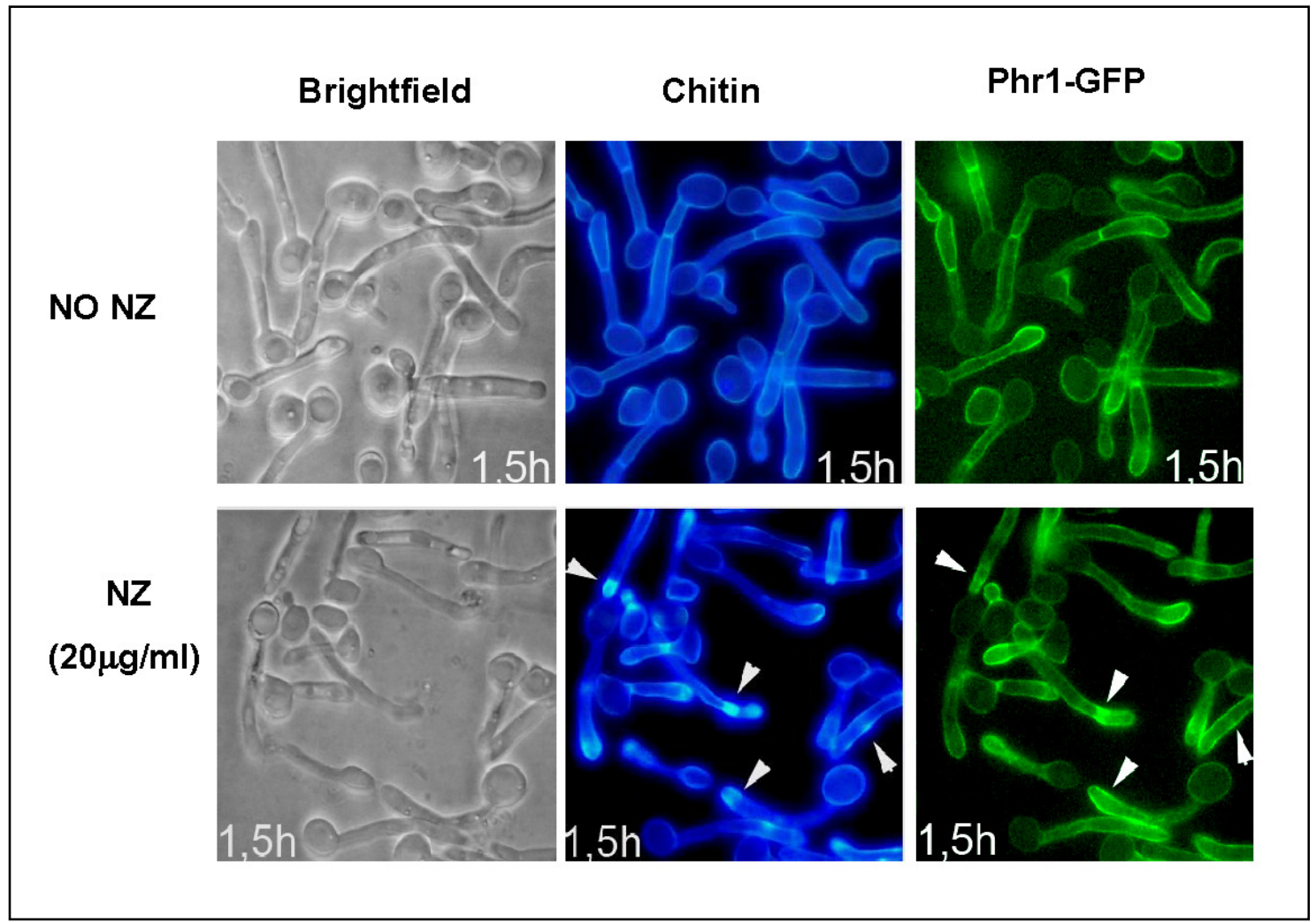

Figure 11. Effect of Nocodazole in Candida albicans hyphal growth. Cells from UBP8 strain hyphae were grown in Medium 199 at $\mathrm{pH} 7.537^{\circ} \mathrm{C}$ and after $2 \mathrm{~h}$ from the induction of the filamentation $20 \mu \mathrm{g} / \mathrm{ml} \mathrm{of} \mathrm{NZ}$ were added to the culture. Cells were collected after $1.5 \mathrm{~h}$ from the addition of the inhibitor. $1 \mu \mathrm{g} / \mathrm{ml} \mathrm{of}$ Calcofluor White was added to the culture for chitin staining during the last 10 minutes of growth. Before microscopy observation cells were washed twice with PBS. Cells growing in the absence of NZ were used as a control (upper panels).

Green fluorescence indicated that in those regions Phr1-GFP did not localized properly being almost impossible to find hyphae with thick green segments that were present in the region of the septa in untreated cells.

Together, all these observations indicate that polarized localization of Phr1-GFP in the tip of the germ tubes, as well as the localization of the protein in the lateral wall of the hypha, does not depend on microtubules whereas a proper polymerization of microtubules is required for proper chitin deposition and localization of Phr1-GFP in the septa.

Actin is required for correct localization of Phr1-GPF during yeast and hyphal growth:

As a first approach to examine the requirement of actin for Phr1 polarization, we did an experiment with cells expressing the Phr1-GFP fusion protein that were incubated overnight in YPD at $30^{\circ} \mathrm{C}$. Cells were then induced to form hyphae (in Medium 199 buffered at 
$\mathrm{pH} 7.5$ at $37^{\circ} \mathrm{C}$ ) in the presence or absent of Cytochalasin A (CA), an inhibitor of actin cable formation. To identify the actin concentration that was efficient to disrupt the organization of the actin cytoskeleton in $C$. albicans cells expressing Phr1-GFP protein, various concentration of $\mathrm{CA}$ ranging from 5 to $20 \mu \mathrm{g} / \mathrm{ml}$ were tested. Effect of CA on actin cytoskeleton was assesed by visualizing the actin cytoskeleton directly in Rhodamine-phalloidine stained cells. As previously reported (Hazan, Sepulveda-Becerra et al. 2002), actin in hyphal cells (assayed by visualization of phalloidin-stained cells) was primarily detected as bright cortical patches associated with the distal region of the growing hyphae (Figure 12A, left panel). Actin cable inhibition by CA was found to be effective at a concentration of $20 \mu \mathrm{g} / \mathrm{ml}$ of CA.

We observed that, independently of the ability to disrupt actin cytoskeleton at all the concentrations, CA inhibited hyphal formation when added in the hyphae inducing medium. So, with this experiment we were not able to address the question of whether Phr1 localization in during hyphal growth depends on actin cytoskeleton. Nevertheless, we observed that in cells growing as yeasts (due to the effect of CA that abolished hyphal growth) treatment with $\mathrm{CA}$ altered Phr1-GFP localization. Phr1-GFP remained in the cytosol as bright internal patches (Figure 12B). Thus, with this experiment we could demonstrate that actin cytoskeleton is essential for correct transport of Phrl protein to the cell surface.

In order to asses whether the localization of Phr1-GFP protein was affected by disruption of actin cable formation in hyphal growing cells, we designed an experiment in which CA was added to the culture $2 \mathrm{~h}$ after the induction of the filamentation. Like in previous experiments stationary-phase cells were induced to form hyphae in Medium 199 buffered at $\mathrm{pH} 7.5$ at $37^{\circ} \mathrm{C}$. In these conditions, different concentrations were added to the hyphal growing cells $2 \mathrm{~h}$ after the induction of the hyphal process. 

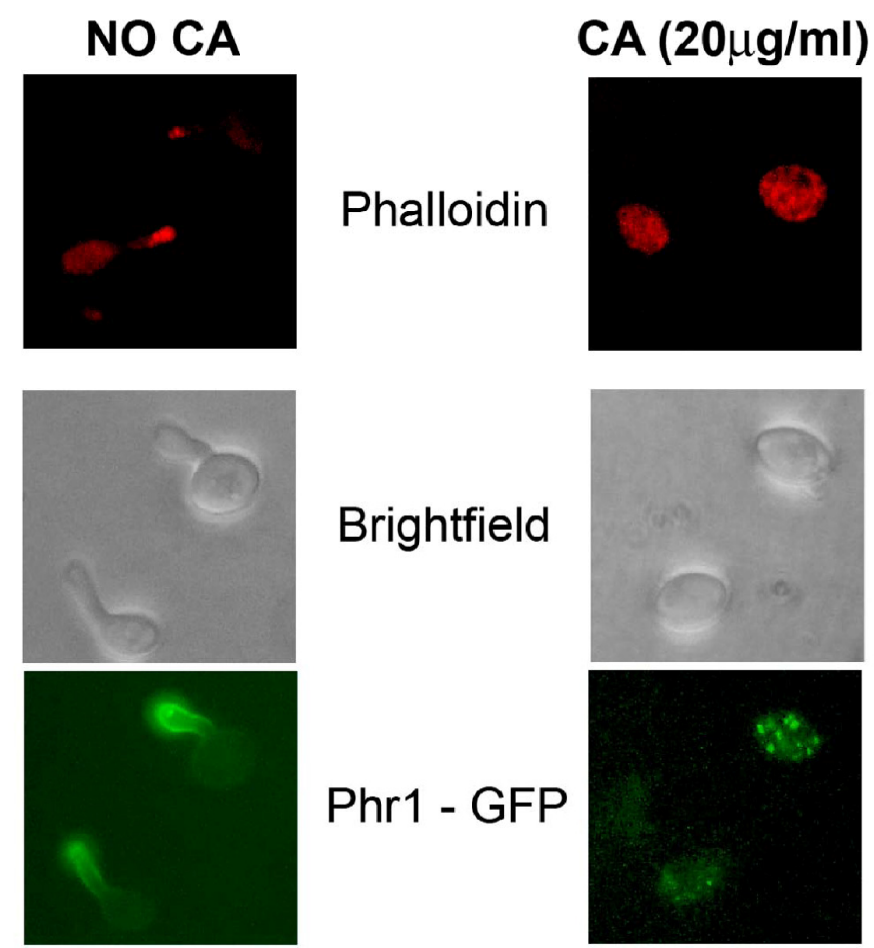

Phr1 - GFP

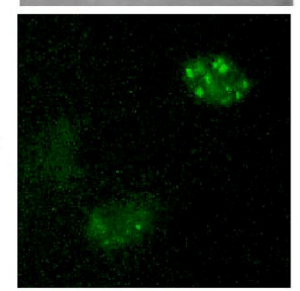

Figure 12. Cythocalasin A abolishes hyphal formation when added directly to the hyphal inducing Media. Cells expressing Phr1-GFP were induced to form hyphae in Medium 199 at $\mathrm{pH}$ 7.5 and $37^{\circ} \mathrm{C}$ with $20 \mu \mathrm{g} / \mathrm{ml}$ of CA. (A) Staining of actin cytoskeleton with Rodhamine phalloidin. (B) Phr1-GFP visualized after 30 minutes from the addition of CA.

We tested concentration of CA ranging from 5 to $20 \mu \mathrm{g} / \mathrm{ml}$ and observed the effect on actin structure by visualizing the actin cystoskeleton with Rhodamin-falloidin. Cells were observed under a fluorescent microscope 30 minutes after the addition of the inhibitor. We did not find differences in the organization of the actin cytoskeleton when $5 \mathrm{mg} / \mathrm{ml}$ were added to the culture (data not shown). In contrast, treatment of cells with $10 \mu \mathrm{g} / \mathrm{ml}$ of CA led to a loss of the bright tip localization pattern of actin patches and in many cases caused a swelling of the tip of the hyphae (data not shown). The effect was more evident when using $20 \mu \mathrm{g} / \mathrm{ml}$ of CA (Figure 13). 


\section{NO CA Brightfield \\ Phalloidin}

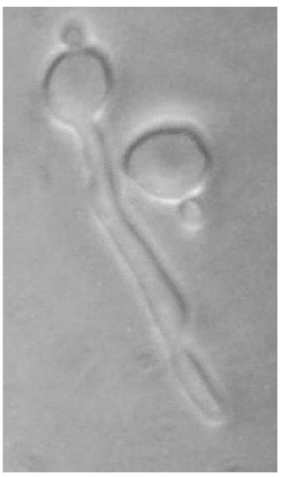

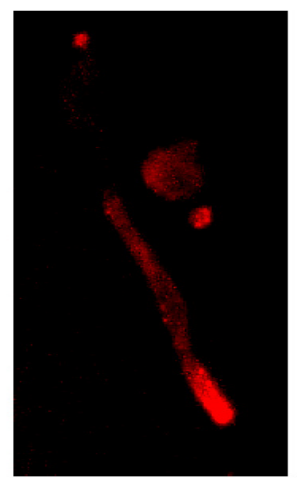

CA $(20 \mu \mathrm{g} / \mathrm{ml})$ Brightfield Phalloidin
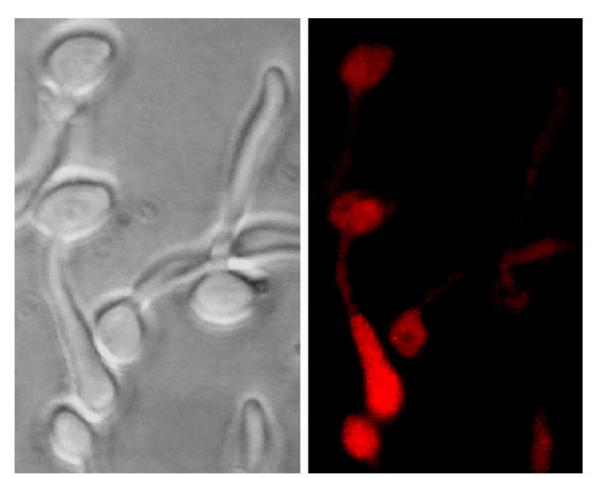

Figure 13. Addition of $20 \mu \mathrm{g} / \mathrm{ml}$ of CA to hyphal growing cells results in the desorganization of the actin cytoskeleton. Cells were induced to form hyphae in Medium $199 \mathrm{pH} 7.5$ at $37^{\circ} \mathrm{C}$ and after $2 \mathrm{~h} 20 \mu \mathrm{g} / \mathrm{ml} \mathrm{of}$ CA were added to the culture. Cells were observed under the fluorescence microscope 30 min after the addition of the inhibitor .

To analyze whether the Phr1-GFP localization was affected by CA treatment we used $20 \mathrm{mg} / \mathrm{ml}$ of CA that were added to the culture $2 \mathrm{~h}$ after hyphal growth induction. Whereas in untreated cells Phr1-GFP localizes in the distal region of the hyphae and is present along the entire surface of the hyphae, in cells treated with CA the Phr1-GFP protein did not accumulate in the tips of the hyphae and also the green fluorescence was less bright in the walls of the hyphae (Figure 14). This defect in the localization of Phr1-GFP protein was already visible when using $10 \mu \mathrm{g} / \mathrm{ml}$. (data not shown). Moreover, in many cells an internal signal in the hyphae was observed with the green fluoresnce remaining as bright internal dots distributed along the hyphae.

Thus, CA treatment affects the polarization of Phr1-GFP protein that does not generate a bright signal in the tips of the hyphae and affects also the distribution of the protein along the walls of the hyphae.

Internal fluorescence in the hyphae in the presence of CA can be interpreted as a consequence of the impaired transport of the newly synthetized protein to the cell surface during hyphal growth and/or as a consequence of an abnormal redistribution of the protein along the membrane from the distal regions. 


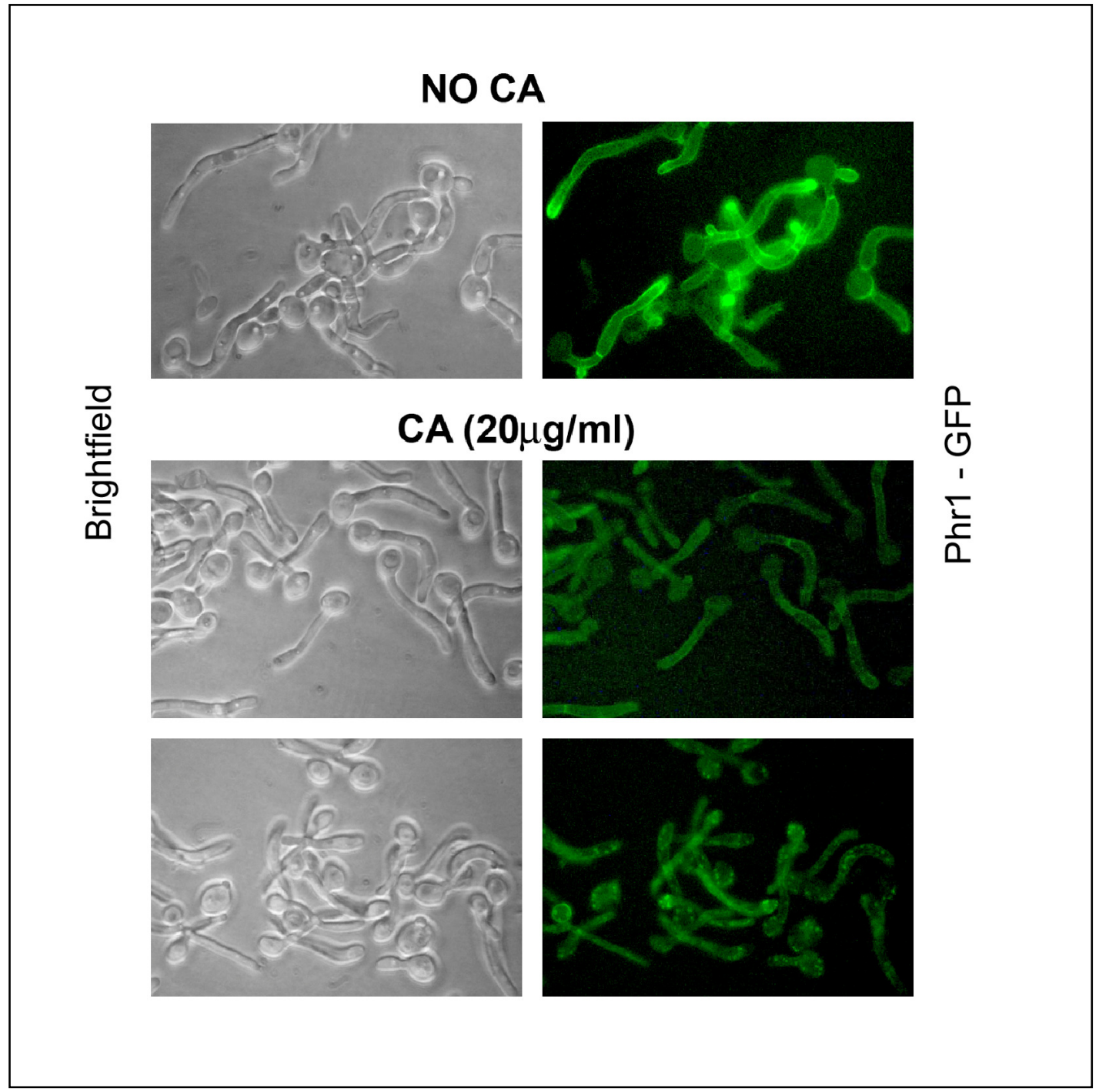

Figure 14. Correct localization of Phr1-GFP in hyphal growing cells requires an inctact actin cytoskeleton. Stationary phase cultures of UBP8 cells were induced to form hyphae in Medium 199 $\mathrm{pH} 7.5$ for $2 \mathrm{~h}$. Cells were then treated with $10 \mu \mathrm{g} / \mathrm{ml}$ of CA for $20 \mathrm{~min}$ (upper panel in treated cells) or $30 \mathrm{~min}$ (bottom panel in treated cells). 
DISCUSSION:

Candida albicans is recognized as one of the most common causes of bloodstream infections, with a high attributable mortality rate (Almirante, Rodriguez et al. 2005; Horn, Neofytos et al. 2009). The limited therapeutic drug arsenal and the emergence of resistant strains make crucial the study of $C$. albicans pathobiology in order to discover new molecular targets for the development of antifungal drugs (Horn, Neofytos et al. 2009). Cell wall and plasma membrane are promising biological structures to look for proteins involved in cell wall biogenesis, hostpathogen interactions and cellular signalling. The study of these proteins might reveal new antifungal targets, diagnostic and prognostic markers as well as vaccine candidates (Roemer, Jiang et al. 2003; Gozalbo, Roig et al. 2004).

Yeast cell walls are dynamic exocellular structures that provide physical and osmotic protection and for this reason they are essential for cell viability. They are constantly changing in architecture and composition during the cell cycle and in response to external stimuli (Klis, Mol et al. 2002; Firon, Lesage et al. 2004). Phr1p of C. albicans is a homologue of Gas1p of $S$. cerevisiae. It is endowed with $\beta$-(1,3)- glucan-transferase activity which is known to be required for proper cell wall assembly (Fonzi 1999). Phr1p is an important virulence factor of C.albicans nevertheless it is still a poorly characterized protein. Thus, the aim of this $\mathrm{PhD}$ work has been the analysis of Phr1p localization in C.albicans both in yeast and hyphal form. In addition, I have analyzed the role of Phrlp in adhesion and invasion of human epithelia as these processes are crucial for the establishment and progression of C. albicans infections (see discussion Chapter II). The results support the notion that Phr1p plays an important role in guiding the apical growth and promoting adhesion and invasion of epithelia. This makes the Phr1 protein a very promising target for antifungal drugs.

It should be recalled that $\mathrm{Phr} / \mathrm{Gas} / \mathrm{Gel}$ proteins are also potent fungal antigens which maybe suitable for the development of new antifungin diagnostic kits (Arroyo, Sarfati et al. 2007). Moreover, it has also been reported that these glycoproteins are effective when used as vaccines for immunotherapy in mouse. (Xue, Hung 2005; Delgado, Xue et al 2003). 
Localization of Phr1-GPF in the lipid rafts:

In this work we have been able to visualize Phr1-GFP in microdomains of the plasma membrane of $C$. albicans during vegetative growth and confirmed biochemically that Phr1-GFP is recovered in the lipid rafts fraction, also named detergent-resistant membranes (DRMs) or sterol- and sphingolipidenriched domains (SRDs). This result is consistent with previous published data from proteomic analysis that identified the wild type Phrlp in DRMs of $C$. albicans (Insenser, Nombela et al. 2006). Lipid rafts are known to be involved in many dynamic cellular processes, like protein sorting, signal transduction, cytokinesis, cell polarity and therefore the proteins sequestered in DRMs are presumed to be important for the fitness of the organism. In $S$. cerevisiae, membrane microdomain clustering at the mating projection is involved in the generation and maintenance of polarity during mating (Bagnat and Simons 2002). A model proposed that lipid and protein polarization result from a rapid endocytotic recycling and slow diffusion in the membrane at the tip of the mating projections (Valdez-Taubas and Pelham 2003). However, other authors argued that septins, which localize at the basis of the mating projections, establish a boundary domain that helps to sequester lipids and proteins at the shmoos tips (Martin and Konopka 2004). A polarization of lipid rafts has been detected in hyphal growth in C. albicans but not in budding or pseudohyphal cells (Alvarez, Douglas et al 2007). Polarity is a matter of crucial importance in C.albicans since hyphal growth is required for full virulence.

The presence of Phrlp in the lipid raft fraction of $C$. albicans and at the tip of germ tubes hyphae, as reported in this thesis, but not at the tip of the bud must be taken into account in this regard. Further analysis of the possible colocalization of Phr1-GFP with a marker of the sterol-rich membranes, such as the fluorescent probe "filipin", will be performed to assess whether Phr1p is sequestered in sterol-rich rafts at the apex of the hyphae. Filipin was also used to show that sterol polarization in hyphal growth is disrupted by latrunculin $\mathrm{A}$, indicating that lipid rafts polarizations requires the actin cytoskeleton.

Interestingly, it has been reported that filipin staining is enriched not only at the hyphal apex but also at septation sites in hyphae where colocalization with septins was observed (Martin and Konopka 2004). This suggests that sterol-rich domain play a role also in septation and 
septins may create a boundary domain to restrict their diffusion. Interestingly, in the present $\mathrm{PhD}$ work Phr1-GFPp was detected also in the septa of hyphae. Future studies will be performed to further analyze whether the localization of Phrlp is coupled to polarization of sterol-rich domains and if agents that inhibit sphingolipid (myriocin) or sterol biosynthesis (ketoconazole) also affect Phr1p localization.

\section{Localization of Phr1-GFP during vegetative growth in $C$. albicans:}

We have observed that in exponentially growing cells Phrl-GFP is present at the cell periphery in agreement with the predicted plasma membrane localization of the protein. Interestingly, it also localizes in the chitin ring, in the septum and in bud scars. In these last three sites Phr1-GFP colocalizes with the chitin. A twodimensional confocal analysis has shown that cells which have completed cytokinesis but not yet the cell separation, the green fluorescence of Phrlp covers all the plane of division at the septum region (data not shown). This indicates that Phrlp is localized at -or very close to- the primary septum. This is consistent with the data obtained for Gas1p (Chapter III and enclosed article).
In future studies, inhibitors of the chitin synthase III (Chs3p), which is responsible for the chitin ring which surrounds the septum and Chs1p, the chitin synthase responsible for the formation of chitin disk in primary septum, will be used to study the effects of perturbation of chitin synthesis on Phrlp localization.

As for Gas1p of $S$. cerevisiae, Phr1p has been considered for many years as a canonical plasma membrane protein. In recent years, the identification of Phr1p among the set of the covalently linked cell wall proteins of C.albicans has been reported (de Groot, de Boer et al. 2004). However, no studies were performed to test whether the cell wall-form of Phr1p is localized at specific sites. Gas1p, the homolog of Phr1p in S. cerevisiae, was also reported to be covalently linked to the cell wall (De Sampaio, Bourdineaud et al. 1999; Yin, de Groot et al. 2005). In other studies of our laboratory, which were carried out in parallel to those of $C$. albicans, Gas1p was detected as covalently bound to the cell wall both to the $\beta(1,3)$-glucan and to chitin (see further Chapter III ). Moreover, Gas1p was found to be specifically cross-linked to the cell wall in the chitin ring, and bud scars which are derived from it, and to the cell wall of secondary septa which lie in close proximity of the primary septum 
(Rolli Ragni et al, 2009). Thus, colocalization of Phrlp with the chitin ring and primary septum raises the possibility that also the cell wall-form of Phrlp undergoes the cross-linking into these sites similarly to what has been reported for Gas1p. A model is shown in Figure 15 (Annex Chapter I).

Thus, we further investigated the potential role of Phrlp in the septum region of vegetative growing cells.

\section{Phr1p is required for strengthening} the neck region during cell division:

It is well known that the lack of PHRI gene results in morphological abnormalities of $C$. albicans cells grown at alkaline $\mathrm{pH}$. Cells become round, enlarged and multibudded after extended growth (Saporito-Irwin, Birse et al. 1995). As in the gas $1 \Delta$ mutant of $S$. cerevisiae, the last trait of the phrld null mutant is likely to be a consequence of defective bud maturation and bud separation. Here, we have shown that the neck region is enlarged in phrls null mutants supporting the idea that Phrlp could play an important role in strengthening the cell wall at the level of the neck region between the mother and daughter cells and also in preventing growth in this area. In wild type cells the diameter of the bud neck is constant during the cell cycle and its high resistance contributes to drive the growth into the bud. Thus, the enlargement of the bud neck size, as occurs in the phrls mutant, could also give origin to defects in bud maturation.

\section{Localization of Phr1-GFP during} filamentation in $C$. albicans and role of Phr1p:

We have also performed a detailed analysis of the localization of the Phr1 protein during hyphal growth. We have found that Phr1-GFP protein concentrates at the tip of the germ tubes at the beginning of the filamentous growth giving origin to a very bright fluorescence. When the germ tubes grow, the fluorescent protein remains at the apex of the hyphae, but it also gradually distributed along the lateral cell walls of the hyphae as they elongate. Phr1 protein is highly polarized during hyphal growth being more abundant in the tip and subapical regions of the hyphae where chitin is less abundant. These results suggest that Phrlp is required at the site of maximal growth probably for the incorporation and elongation of newly synthesized $\beta$-(1,3)glucan in the expanding cell wall. This function could be synergic with the 
action of $\beta$-(1,3)-glucan synthase Fks1p, the enzyme responsible for the production of $\beta-(1,3)$-glucan in $C$. albicans. In these sites of highly polarized growth chitin is absent as the cell wall needs to be more elastic and expandable. When Phr1-GFP distributes along the lateral cell walls of the hyphae, it probably contributes in the formation of cross-links with other cell wall components, such as chitin or mannoproteins, and altogether confer a high mechanical resistance to the cell walls which contributes to drive the growth toward the apical region. Strikingly, the tips of the hyphae are still bright as hyphae elongate but at the same time the fluorescence becomes more visible along the length of the hyphae. Thus, at longer time from hypha induction Phr1-GFP label the entire periphery of the hyphae.

This pattern of localization of Phr1GFP could be a consequence of the apical transport of vesicles from the Golgi to the tip of the germ tubes and hyphae. Afterwards the transport of the vesicles might exhibit a longitudinal pattern along the walls of the hyphae. Alternatively, the lateral distribution of Phrlp could be explained also in terms of lateral mobility within the lipid bilayer of the plasma membrane thanks to the GPI-anchor that confers mobility to the protein and to the movement of the lipid rafts. However, it has been reported that the majority of the Golgi complex is redistributed to and maintained at the distal portion of the hyphae near the growing apical tip (Rida, Nishikawa et al. 2006). Therefore the redistribution of Phrlp along the hyphae is more likely to be due to the lateral mobility through the membrane GPI-anchor.

As mentioned above, Phrlp also shows an additional localization: it is present as a thin line along the septa of the hyphae, indicating a potential role of the protein also at this site. Also in the hypha the presence of Phrlp at the septation sites might be related to the need of increasing locally the cell wall resistance.

\section{Role of cytoskeleton in the polarized localization of Phr1p:}

The mechanisms that establish and maintain apical growth during hyphal formation in $C$. albicans are not well understood. However, based on the knowledge of how polarized growth occurs in other systems, it is generally accepted to involve two possible mechanisms: (i) the asymmetric deposition of membrane and cell wall components to the growing tip could 
result from endocytotic recycling and the slow diffusion of membrane or (ii) the septins could establish a boundary domain that help to sequester proteins and sterol-rich domains and maintain the polarization to the apical region (Rambourg, Jackson et al. 2001; Pruyne, Legesse-Miller et al. 2004). The process of budding in Saccharomyces cerevisiae requires many proteins that regulate the site selection, reorganization of the actin cytoskeleton, and polarization of the secretory apparatus (Pruyne, LegesseMiller et al. 2004). Hyphal growth in $C$. albicans presents an additional challenge owing to the requirement to rapidly deliver of membrane and cell wall materials over a long distance. In $S$. cerevisiae, polarization and targeting of post-Golgi vesicles to the selected growth site is an actin-based process and does not require microtubules. Although the actin cytoskeleton is essential for hyphal formation in C. albicans, there are conflicting reports on the role of microtubules in this process (Yokoyama, Kaji et al. 1990; Akashi, Kanbe et al. 1994). In other filamentous fungi, microtubules are important for hyphal growth (Raudaskoski, Mao et al., 1994; Steinberg Wedlich-Söldner at al., 2001). It has been proposed that they are responsible for the long distance transport of post-Golgi secretory vesicles to the Spitzenkörper, whereas actin filaments controls short-range vesicle transport form the Spitzenkörper to the plasma membrane (Crampin, Finley et al. 2005). However, a study of the role of the secretory pathway and cytoskeleton during hyphal formation in C. albicans, published by Rida et al. in 2006, has demonstrated that microtubules do not play a role in transporting or in maintaining the Golgi Apparatus near the growing hyphal tip (Rida, Nishikawa et al. 2006).

Using Nocodazole (NZ), an inhibitor of microtubule polymerization, we have shown in this work that Phr1-GFP polarization and distribution along the hyphae do not depend on microtubule function. These results are in accordance with those regarding the independence of the Golgi vesicles transport reported above. However, we observed that localization of the protein in the septa of the hyphae is altered when the microtubules are depolymerized. It should be marked that in that case chitin deposition in the septa in this conditions is considerably altered and giving the fact that Phr1-GFP protein colocalizes with chitin in that area, mislocalization of Phrlp in the septa could not be directly linked to microtubule disfunction but more likely with the unproper depositon of chitin in that area. 
Our results also indicate that proper localization of Phrlp during hyphal growth depends on a correct organization of actin cytoskeleton. Extensive genetic evidence demonstrates that proper assembly and polarization of the actin cytoskeleton are essential for polarized fungal cell growth. The role of actin cytoskeleton in hyphal formation in C. albicans has also been demonstrated (Akashi, Kanbe et al. 1994). Moreover, it has been shown that the maintenance of the Golgi at the distal portion of hyphal cells and the vesicles motility to the tip of the hyphae requires an intact actin cytoskeleton (Rida, Nishikawa et al. 2006; Veses and Gow 2008). So, our findings suggest that abnormal localization of Phrlp in hyphal cells treated with Cytochalasin A, an inhibitor of actin cable formation, is probably due to theinability of the cells to maintain the Golgi at the distal portions as well as to failure in the transport of secretory vesicles to the cell surface. The fact that in our experiments a small fraction of the protein was found in the lateral cell walls could be a consequence of the fact that the inhibitor of the actin cable formation was added $2 \mathrm{~h}$ after the induction of filamentation. During this time Phrlp was localized in the tips of the hyphae as normally occurs and afterwards, when the organization of the actin cytoskeleton was altered, the fraction of the protein that was deposited in the tips of the hyphae before the addition of the inhibitor could distribute along the membrane. 


\section{MODEL PROPOSED FOR THE LOCALIZAZION OF Phr1p DURING VEGETATIVE GROWTH IN C. albicans:}

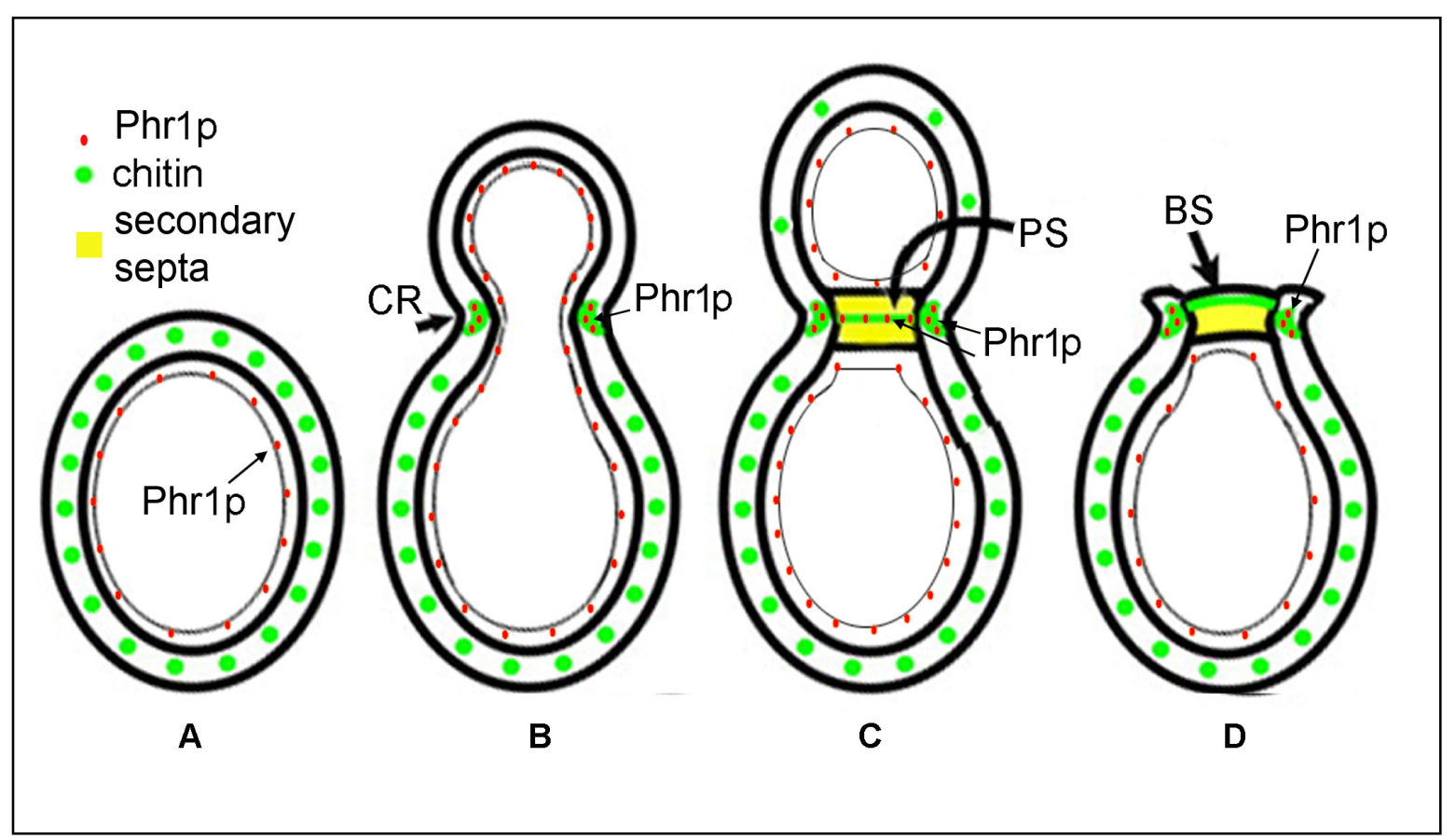

Figure 15. Scheme summarizing the localization of Phr1p in $C$. albicans vegetative cells. (A) In a new born cell Phr1p is in microdomains of the plasma membrane. (B) In a small-budded cell, Phr1p is in the plasma membrane but is also cross-linked to the chitin ring presumably through a transglycoylase reaction. (C) In large budded cells Phr1p is localized to or in close proximity to the primary septum. (D) After cell separation, Phrlp remains in the bud scar on the mother cell side and in the plasma membrane. CR: Chitin Ring synthesized by $C a C h s 3 p$; PS: Primary Septum produced by $C a C h s 1 p$ : BS: Bud Scar. 
CHARACTERIZATION OF THE phr1s MUTANT PHENOTYPE. DEFECTS IN ADHESION AND INVASION IN RECONSTITUTED HUMAN EPITHELIAL MODELS:

\section{BACKGROUND:}

The ability to grow in a wide range of ambient $\mathrm{pH}$ is crucial for Candida albicans to survive in different host niches and for its success as a pathogen. Several C. albicans genes are regulated by the extracellular $\mathrm{pH}$ through Rim101dependent pathways (Bensen, Martin et al. 2004). PHRl is one of these $\mathrm{pH}$ regulated genes.

As we have already mentioned in this thesis, in vitro, the homozygous null mutant of PHRI exhibits $\mathrm{pH}$-conditional morphological defects (see pages 36 and 37 on Introduction). It is known that at alkaline $\mathrm{pH}$ values, the mutant, unlike the parental strain, is unable to conduct apical growth either in the yeast or hyphal forms (Saporito-Irwin, Birse et al. 1995). We have also observed that the defects in filamentation on solid media a are stronger than in liquid media. As shown in the Annex on this chapter phrls mutant is completely unable to form hyphae on solid media at alkaline $\mathrm{pH}$.
It is known that in vivo PHRl gene is required for virulence. The defects in virulence of the phrls are $\mathrm{pH}$-dependent defects.. Studies performed using a mouse model of systemic candidiasis by Fonzi and col. (Ghannoum, Spellberg et al. 1995) demonstrated that phrls null mutant is avirulent. The effect on virulence was not associated with differences in the viability of the strains as both the mutant and the revertant strains presented a similar viability in vitro. The reduced virulence was neither associated with a rapid clearance of host tissue. In another study published later by Fonzi and col. (De Bernardis, Muhlschlegel et al. 1998) it was demonstrated that the virulence phenotype of a PHRI null mutant paralleled the $\mathrm{pH}$ dependence in vitro phenotype. Since vaginal $\mathrm{pH}$ is around 4.5, phrl $\Delta$ mutant was found to be uncompromised in its ability to cause vaginal infection but was avirulent in systemic infection which regards the diffusion into the bloodstream, a compartment with a slightly alkaline $\mathrm{pH}$ (around 7.4). Moreover the virulence of a heterozygous strain $(\mathrm{Phr} 1+/-)$ was indermediate indicating the possibility of a gene dosage effect. 
The first step during host-pathogen interactions is attachment of the microorganism to epithelial surfaces of the host followed by invasion into deeper tissues. During mucosal infection, $C$. albicans invades the oral mucosa and persists within the epithelium causing superficial lesions. Normally, fungal proliferation, colonization and invasion are hindered by dense epithelial layers with high turnover rates of cell renewal and innate defence mechanism such as the secretion of antimicrobial compounds. However, C. albicans has evolved several strategies to overcome these defence mechanism for both commensal growth and infections. These attributes allow not only the development of superficial infections, but also disseminations via the blood system and the invasion of virtually every organ of the human host.

Remodelling the cell wall architecture is critical to preserve cellular integrity in response to environmental and stress conditions including challenge with antifungal drugs. In addition, the dynamic nature of the cell surface alters the physical properties of the fungal-host interaface and thereby influences adhesion to the host and recognition by components of the host's immune system.

\section{OBJECTIVES:}

In order to better understand the role of Phrlp in the pathogenesis of Candida albicans, we wanted to investigate whether phrls null mutant was affected in the early stages of the host-pathogen interactions. In particular the aims of our work were to analyze the ability of the mutant to: (1) adhere to both biotic and abiotic surfaces and (2) invade human tissues using reconstituted human epithelial models (RHE). For that purpose I moved to the Fraunhofer Institut in Stuttgart (Germany) where I worked in the group of Dr. Steffen Rupp.

\section{RESULTS:}

Phr1s mutant is defective in adhesion to abiotic surfaces:

Candidiasis is often initiated by the colonization of inert surfaces. Using an in vitro adhesion assay we wanted to determine whether deletion of PHRI alters the ability of $C$. albicans to adhere to plastic surface. Experiments were performed in Stuttgart using the assay that was previously described by Rupp et al. (Sohn, Senyurek et al. 2006). Since the assays were establish to analyze the behaviour of Candida albicans in the adhesion of different surfaces including confluent monolayers of human 
epithelial cell lines, the experiments were set up at $37^{\circ} \mathrm{C}$ under $5 \% \mathrm{CO}_{2}$ using D-MEM supplemented with $10 \%$ fetal calf serum, which is the preferred culture medium for growth and propagation of most mammalian cell lines. It is known that these conditions also induce the morphogenetic switch from yeast to hyphal growth in Candida albicans. We first checked that phrls mutant showed its defective phenotype in the condition that would be used for the assays. The strains used were: the PHRI deleted strain (CAS10), the parental strain (CAI10) and the reconstituted strain (CAS11).

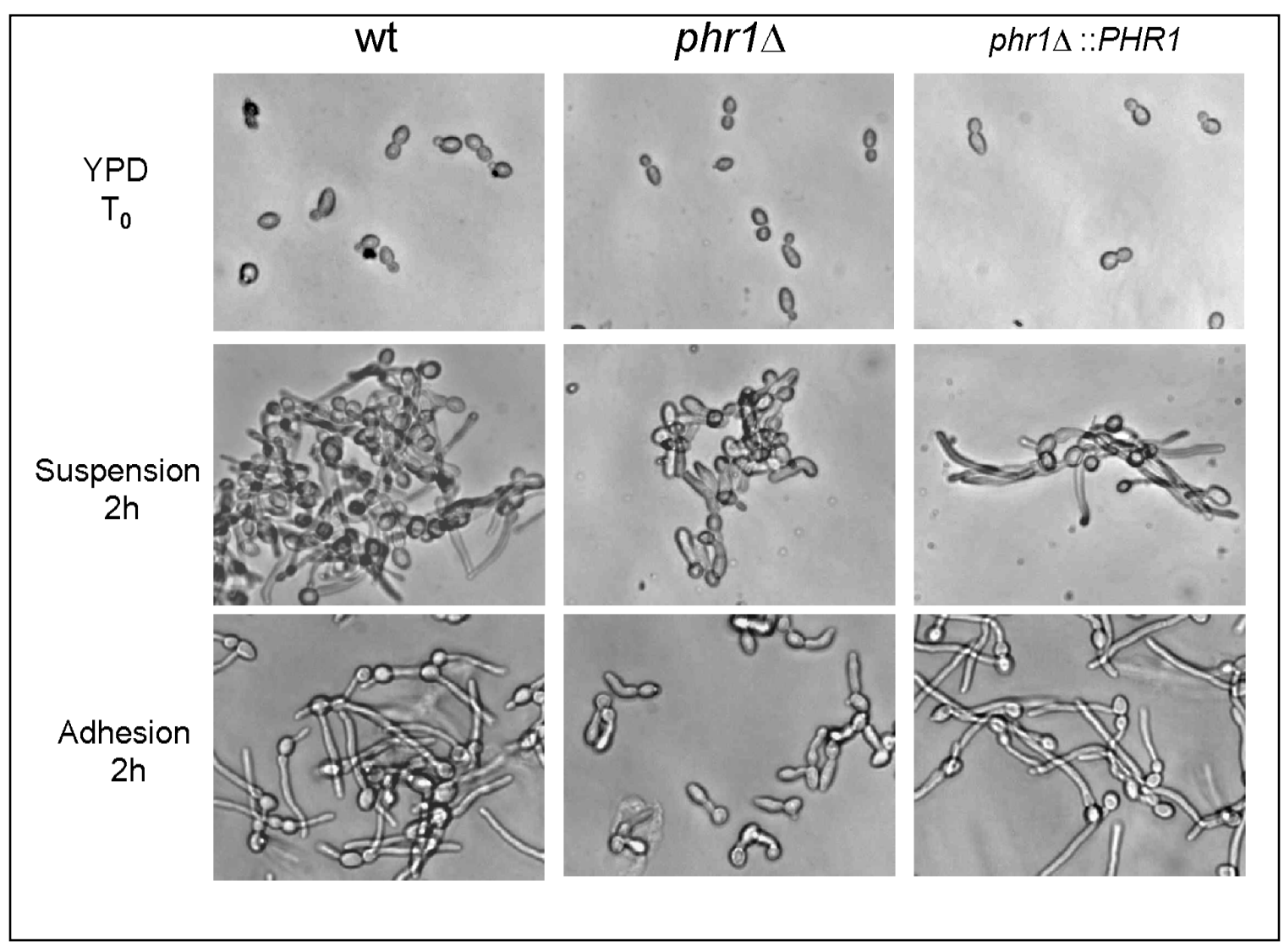

Figure 1. Micrographs of cells of the phrls strain, as well as those for the wild type and the revertant strain growing in erlenmeyers flask in YPD buffered at $\mathrm{pH} 6$ at $30^{\circ} \mathrm{C}$ and after $2 \mathrm{~h}$ of growth in suspension in D-MEM at $37^{\circ} \mathrm{C}$ and $5 \% \mathrm{CO}_{2}$ with shacking or after $2 \mathrm{~h}$ of growth in 24-well-polystyrene-plates with D$\mathrm{MEM}$ at $37^{\circ} \mathrm{C}$ and $5 \% \mathrm{CO}_{2}$.

We found that the mutant was able to develop germ tubes (Figure 1), an indication of the commitment of the cells to the hyphal program, but was defective in elongating them. This corresponds to the typical aberrant phenotype that has been extensively described for the growth under filament inducing conditions. Adhesion assays were performed as described in Materials and 
Methods using 24-well-polystyrene plates. In order to avoid the influence of PHR2 expression, cells were pre-growth in YDP buffered al $\mathrm{pH}$ 6.0. After an overnight culture, $3 \times 10^{2}$ cells (in $50 \mu \mathrm{l}$ ) were added to the each well (for 5 time points in duplicate for each strain) that contained pre-warmed supplemented DMEM medium. Plates were incubated at $37^{\circ} \mathrm{C}$ under $5 \% \mathrm{CO}_{2}$ and at different time intervals (30, 60, 120 and $240 \mathrm{~min})$ non adherent and adherent cells were plated onto YPD plates that were then incubated at $30^{\circ} \mathrm{C}$ for two days. Numbers of $\mathrm{CFU}$ were counted for both adherent and non-adherent cells for each time point in duplicate. The mean values and percentage of adherent cells from two independent experiments were calculated.

A

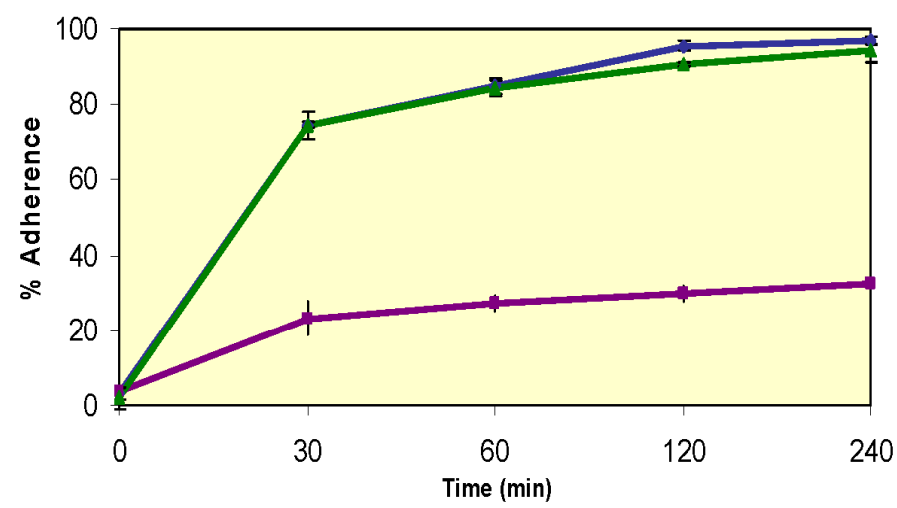

B

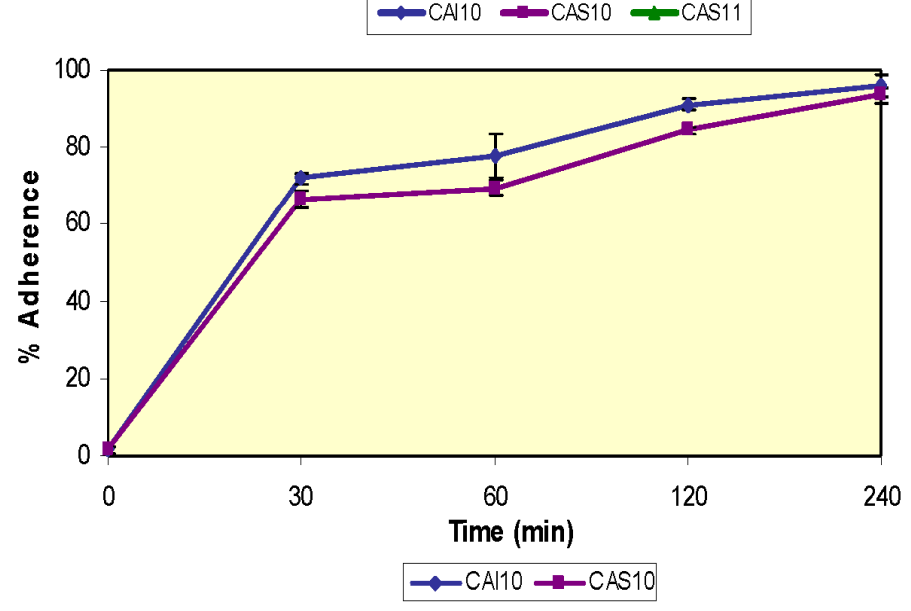

Figure 2. A. Graphics showing the percentage of adhesion of cells from the wild type, phrl $\Delta$ mutant and the reconstituted strain growing in 24-well-polysterine-plates with D-MEM at $37^{\circ} \mathrm{C}$ and $5 \% \mathrm{CO}_{2}$ after 30 , 60, 120 and 240 minutes from the incubation. B. Graphics of the percentage of adhesive cells from the wild type and the phrl $\Delta$ mutant cells growing in 24-well-polysterine-plates with D-MEM buffered at $\mathrm{pH}$ 6 at $37^{\circ} \mathrm{C}$ and $5 \% \mathrm{CO}_{2}$. Percentage of adhesion were calculated from two independent experiments and in each one the data from adherent and non-adherent cells were collected in duplicate. Error bars indicate the s.d. for each set of experiments. 
As shown in Fig 2A, the adhesion of wild type strain (CAI10) was very rapid and occurred with high efficiency. In contrast, adhesion of the phrls mutant strain (CAS10) was notably reduced. The defect in adhesion was already detected after $30 \mathrm{~min}$ as $C$. albicans the wild type reached a value of about $75 \%$ of adhesion while the value of the mutant was about 20\%. Maximum adhesion occurred after $2 \mathrm{~h}$ resulting in approximately $95 \%$ of cells adhering to the substrate in the wild type whereas the percentage of adhesion of the phrls mutant strain reached a maximum value of about $30 \%$. The efficiency and values of adhesion of the reconstituted strain (CAS11) were like that of the wild type indicating that the reintroduction of the PHRI allele fully restores the levels and kinetics of adhesion of the wild type.

As an additional control, we also performed the experiments in the same condition but using supplemented DMEM medium buffered at $\mathrm{pH}$ 6.0, a $\mathrm{pH}$ at which the phenotype of phrld is not yet manifested and $P H R 2$ expression is at a minimum level. At pH6 the adhesion of wild type cells still occurred with a kinetics similar to $\mathrm{pH} 7.5$, indicating that the acidic $\mathrm{pH}$ does not influence this property. We did not find significant differences in the adhesion behaviour of phrls mutant and the wild type strain (Figure 2B) indicating that the defect in adhesion we reported at $\mathrm{pH} 7.5$ was specifically associated to the PHRI deletion and not to other mutations present in the deleted strain.

Loss of Phr1p reduces adhesion of Candida albicans cells to monolayers of epithelial cell lines:

To test the ability of phrls strain of Candida albicans to adhere to human epithelium the assay was similar to that used for the abiotic surface but in this case the cavities of the 24-well-plates were pre-treated to establish a confluent monolayer of epithelial cell lines. Two cell lines were tested: the intestinal epithelial cell line $\mathrm{Caco} 2$ and the oral epithelial model cell line TR146.

In the assay, which was carried out as described in Materials and Methods, the $\mathrm{pH}$ of the medium was around 7.5, as required for the cell lines, and this was appropriate to test the behaviour of phrl $\Delta$ mutant because this is a $\mathrm{pH}$ value at which the PHRl gene is expressed at a high level.

Figure 3 shows the kinetics of adhesion of the three strains used. Attachment of $C$. albicans wild type and reconstituted strains to both $\mathrm{Caco} 2$ and 
TR146 cell lines occurred with a slightly slower kinetic and was less efficient compared with adhesion to plastic. This is in agreement with what has been reported about adhesion of $C$. albicans to abiotic surfaces that is more efficient than the adhesion to monolayers of epithelial cell lines (Sohn, Senyurek et al. 2006).

As shown in Figures $3 \mathrm{~A}$ and $\mathrm{B}$, kinetics of adhesion of the wild type strain were similar for both $\mathrm{Caco} 2$ and TR146 cell lines. In the wild type maximum adhesion occurred after 2-4 h of incubation reaching percentages of adhesion of about $95 \%$ after $4 \mathrm{~h}$ of incubation. The ability to adhere of the phrls mutant strain to both Caco2 and TR146 cells was clearly reduced compared to the wild type strain reaching maximum values of about $30 \%$

A

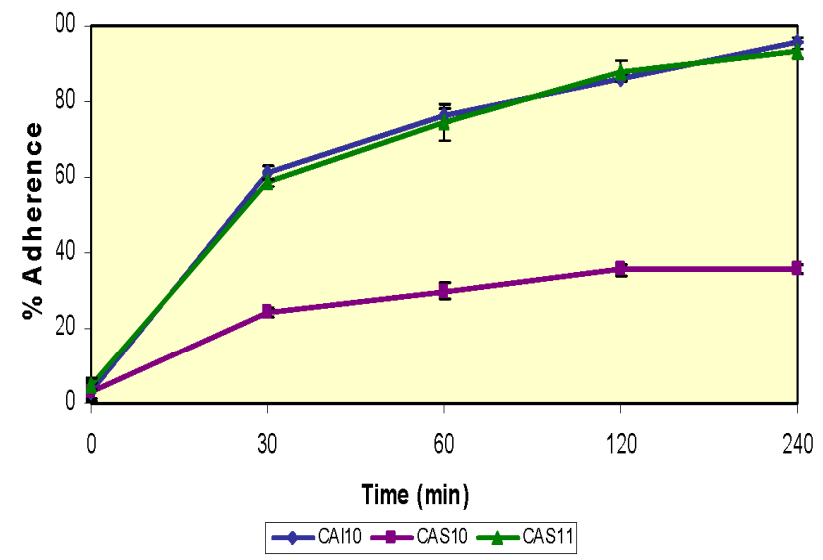

B

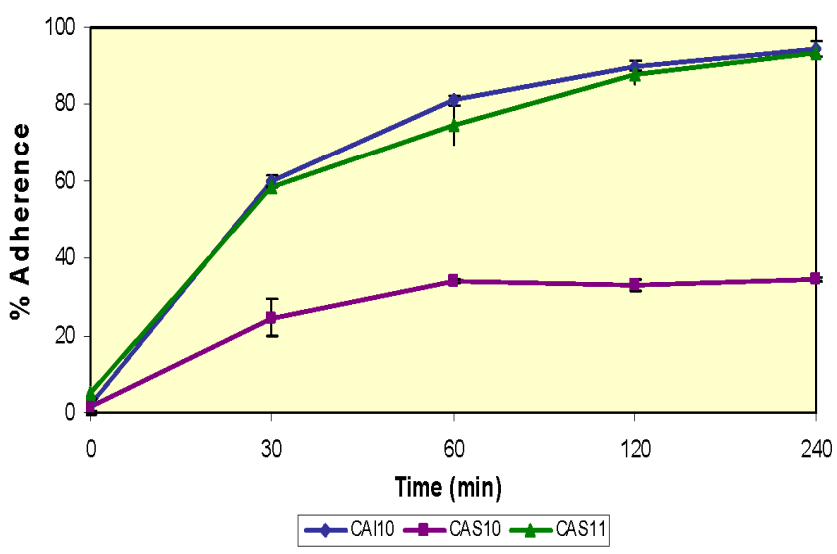

Figure 3. Graphics showing the percentage of adhesion of cells from the wild type, phrl $\Delta$ mutant and the reconstituted strain growing in 24-well-polysterine-plates coated with a monolayer of the intestinal epithelial cell line Caco2 (A) and the oral epithelial model cell line TR146 (B) with D-MEM at $37^{\circ} \mathrm{C}$ and $5 \% \mathrm{CO}_{2}$ after 30, 60, 120 and 240 minutes from the incubation. Percentage of adhesion were calculated from two independent experiments and in each one the data from adherent and non-adherent cells were collected in duplicate. Error bars indicate the s.d. for each set of experiments. 
The defect in adhesion was already evident at the 30min from the incubation of the cell monolayers with $C$. albicans PHR1 deleted cells. After this time, the isogenic strain reached a value of about $60 \%$ whereas the phrls mutant showed a level of adhesion of about $25 \%$.

The PHRI reconstituted strain (CAS11) rescued the defects in adhesion showing percentages similar to those of the wild type strain indicating that the defects of the mutant strains where due to the lack of the PHRI gene.

Expression of adhesion and hyphaspecific genes is affected in phr1s mutant during adhesion to an abiotic surface:

We monitored the expression of a series of selected genes by qRT-PCR. Total RNA was isolated from cultures pre-grown to stationary phase in YPD buffered at $\mathrm{pH} 6$ (time zero) and after $2 \mathrm{~h}$ from the shift to conditions of adhesion on polysterene surfaces. We examined the morphologies of the cells after $2 \mathrm{~h}$ of incubation in adhesion conditions. Strains CAI-10 displayed elongated and thin hyphae whereas PHRI null mutant cells exhibited short and enlarged germ tubes (Figure 4) in agreement with the previously described phenotype (Saporito-Irwin, Birse et al. 1995)
(Figure 6 in Annex of this Chapter). The reintroduction of the wild type allele of PHR1 gene fully complemented the defects in morphology of the strain (Figure 4).

We assayed the expression of two cell-surface adhesin-encoding genes, $A L S 3$ and EAP1. ALS3 is a member of the agglutinin-like $A L S$ gene family which is known to encode differentially regulated cell surface glycoproteins that promote fungal adhesion (see as a review (Chaffin, 2008). ALS3 is specifically expressed during hyphal development and Als3p is consistently distributed on the germ tube and hypha surfaces (Coleman et al., 2009; Hoyer et al., 1998). We also analyzed the expression of EAP1, a gene that it is required for binding of C.albicans cells to polysterene surfaces and to human epithelial cells ( $\mathrm{Li}$ et al., 2007). In addition to these genes, we tested the expression of two hyphal growthassociated genes, HWPI and ECE1. HWPl is thought to be a target of mammalian transglutaminases and is required for adhesion to mammalian cell surface (Chaffin, 2008). ECE1 is highly expressed during hypha formation and its expression increases with the extent of elongation of the cell (Chaffin, 2008; Li and Palecek, 2003; Li and Palecek, 2008). Whereas lack of $H W P 1$ abolishes 
hyphal development, the deletion of ECE1 is not essential for cell elongation or hyphae formation (Chaffin, 2008). The products of all these genes are GPIanchored proteins.

As shown in Figure 4, ALS3 and EAP1 transcript levels were not affected by the PHRI deletion as they were approximately at the same values as the wild type strain (Fig. 5). Since $A L S 3$ is a hyphae specific gene, upregulation of
$A L S 3$ in the condition of adhesion in the phrls cells could be a consequence of the activation of the hyphal program in the mutant. Interestingly, deletion of the PHRI gene caused a marked reduction of $H W P 1$ and ECEl transcripts (Figure 4). The transcript levels of $H W P I$ and ECE1 underwent a decrease of about $60 \%$ and $65 \%$ respectively, if compared to the levels present in the isogenic strain.

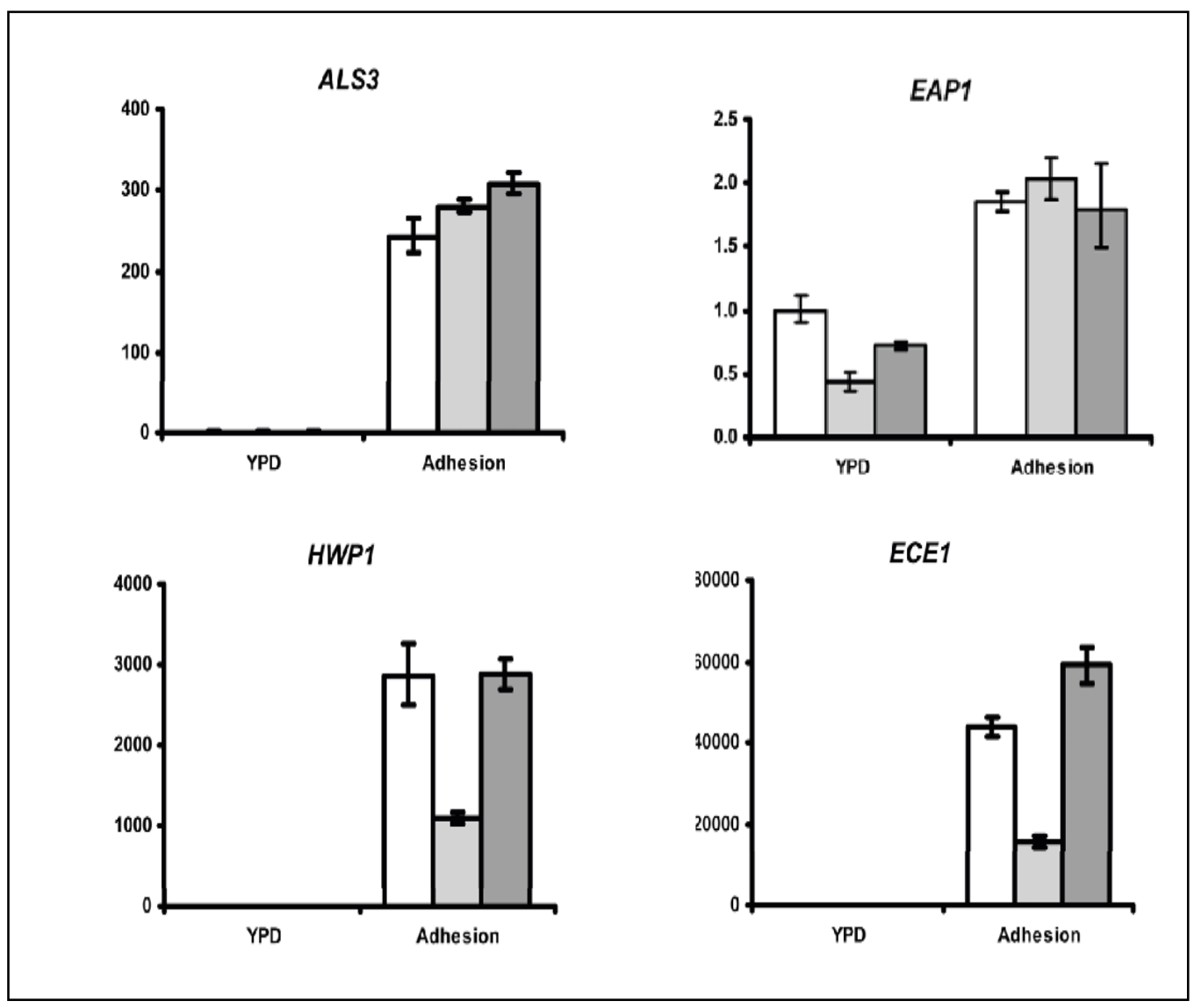

Figure 4. Modulation of gene expression in adhering $\Delta p h r l$ cells. Transcriptional profiling wild type strain CAI-10 (empty bars), $\Delta p h r l$ mutant strain CAS-10 (light grey) and its respective phrl::PHRl revertant strain CAS-11 (dark grey) after $2 \mathrm{~h}$ of adhesion to polystyrene surface under hyphae-inducing conditions. Gene regulation is related to the mRNA level of wild type strain grown in YPD culture. Error bars are estimated for data in triplicate. In total, three independent experiments were performed and the results of a representative one is shown. As a reference control gene TDH3 encoding glyceraldehyde-3-phosphate dehydrogenase was used. 
Reintroduction of the wild type allele restored the wild type levels. In addition, a similar modulation of expression for these genes was present in cells growing in suspension suggesting that the effect is due to the culture conditions and is not specific to the adhesion condition (data not shown)

These results suggest that the morphogenetic defects of the PHRI null mutant activate regulatory pathways that strongly inhibit the expression of hyphal associated genes, HWPI and ECEl, while do not act on the expression of the adhesin-encoding genes $A L S 3$ and EAPI.

Phr1s mutant is unable to invade reconstituted human epithelia:

To investigate the contribution of PHRl gene to invasion of human epithelia we used a reconstituted human epithelial model (RHE) that was developed in Rupp's laboratory in Stuttgart (Hernandez and Rupp 2009). In particular, we used a simple in vitro model in which the initial steps of the host-pathogen interactions are mimicked. The protocol used for these experiments is explained in Materials and Methods and basically consists in the addition of a number of Candida albicans cells (about $2.8 \times 10^{4}$ cells) to a confluent monolayer of Caco2 cells (an intestinal epithelial cell line) that had been built up on a collagen gel matrix. Inserts containing the collagen and the monolayer of $\mathrm{Caco} 2$ cells were then incubated at $37^{\circ} \mathrm{C}$ in an incubator under $5 \% \mathrm{CO}_{2}$. After 7 and $24 \mathrm{~h}$ of incubation, the tissue inserts were removed and subjected to routine histological processing.

Figure 5 shows the images obtained with the histological stained sections that were obtained after 7 and $24 \mathrm{~h}$ from the infection. As shown in Figure 5a, after $24 \mathrm{~h}$ that the wild type strain (CAI10) penetrate into the entire epithelial cell layer very efficiently and invades the collagen matrix. Figure $5 b$ shows that the phrls mutant strain (CAS10) is clearly defected either in penetration into the epithelium or invasion of the inner collagen matrix. The reconstituted strain (CAS11) was able to penetrate into the model tissue and invade the collagen. However, and as observed in three different experiments, it seems to invade less efficiently than the parental strain. (Fig 5c). With this model is difficult to measure epithelial cell damage because the monolayer of the Caco2 cells can appear broken because total confluent was not totally reached during the construction of the inserts or due to the processing of the samples during histologic treatment. Nevertheless, it 
seems that the wild type, unlike the mutant strain, is able invade and to cause damage to the epithelia. The reconstituted strain is able to invade the epithelia but the efficiency is a bit lower compared to the wild type and it is not able to cause epithelial cell damage indicating a gene dosage effect.

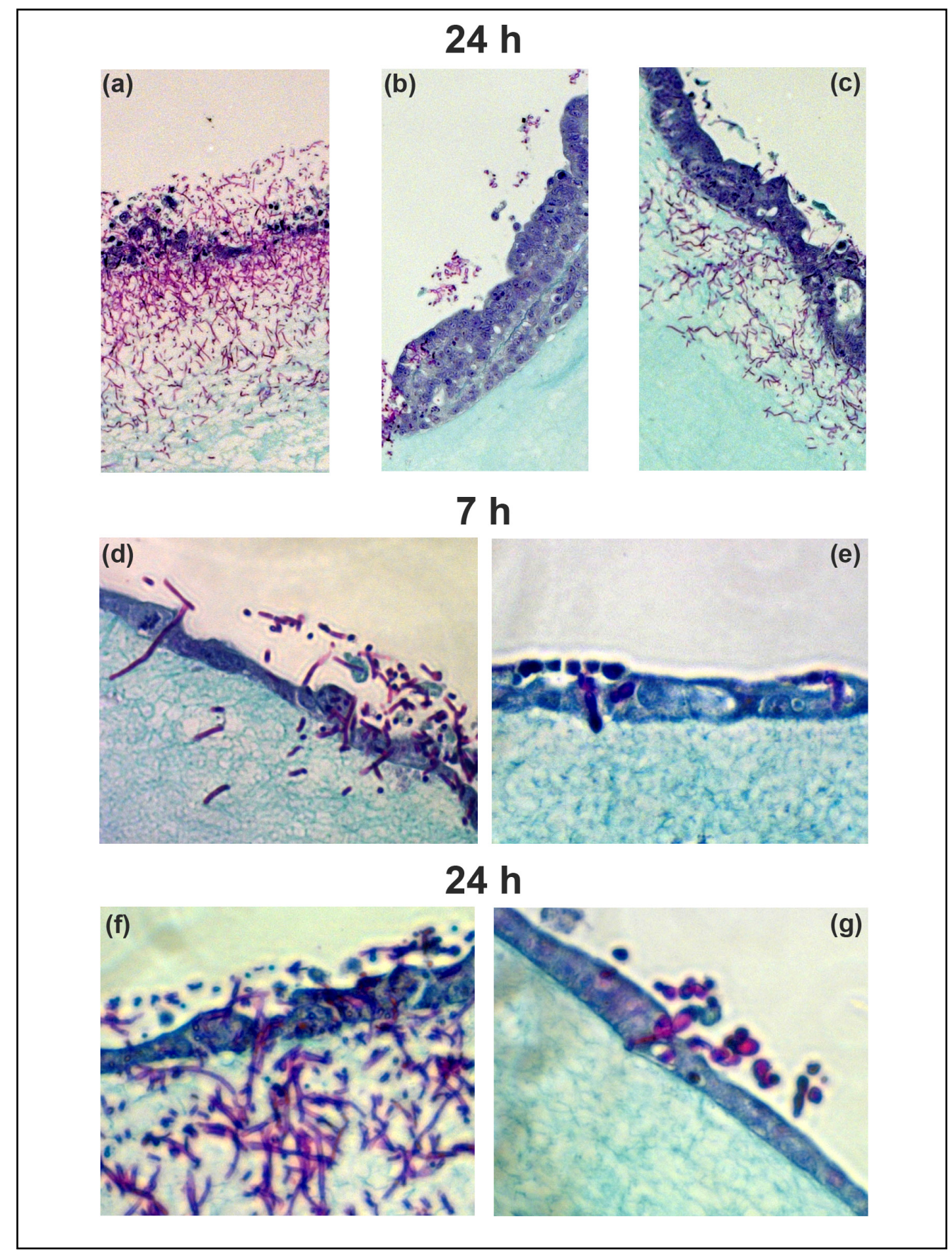

Figure 5. (a to c) Infection of the reconstituted epithelial model (RHE) in which a monolayer of Caco 2 cells is laid on a collagen matrix with wild type (CAI10), phrls (CAS10) and the reconstituted strain (CAS11) after 24h from the infection. (d to g) Magnification images of the infection of the wild type and the phrl $\Delta$ strain after 7 and ( $\mathrm{d}$ and e) and $24 \mathrm{~h}$ (f and $\mathrm{g}$ ). 
In order to analyze with more detail the differences in invasion of the phrls mutant strain with that of the wild type strain we observed the cell morphologies of the strains tested. Figures $5 \mathrm{~d}$ to $\mathrm{g}$ show images of higher magnification of the histological staining after 7 and $24 \mathrm{~h}$. As shown in Figure 5d, after 7 h the wild type strain (CAI10) had already formed long hyphae and penetrated the monolayer of $\mathrm{Caco} 2$ cells. In this early time point most of the wild type Candida hyphae are present inside the epithelial monolayer and some of them have already reached the collagen matrix. By contrast, cells from phrls mutant strain (CAS10) appeared round and with the peanut-shape characteristic of the mutant growing under hyphae inducing condicitions. They were rarely found attached to the Caco2 monolayer. Only in some specific areas they were forming aggregates and occasionally penetrate into the epithelium without reaching the collagen matrix (Figure 5e). After 24h, cells from strain CAI10 and CAS11 had penetrated through the epithelial cell monolayer reaching the collagen matrix. As shown in Figure $2 \mathrm{f}$ and $\mathrm{g}$, most of the hyphae formed by CAI10 strain (Fig. 5f) had invade the epithelia and are present in the collagen matrix. By contrast, cells from CAS10 $(p h r 1 \Delta)$ strain did not produce hyphae and remained with distorted growth projections and peanut-shaped morphologies (Fig. 5g) and did not penetrated into the collagen matrix even if some of them seemed to entry into the epithelium.

\section{DISCUSSION:}

The results reported in this work indicate that the ability to adhere to a plastic surface is strongly reduced in the phrls mutant. Adhesion and hyphal morphogenesis are two tightly linked processes in Candida albicans that contribute to the establishment and progression of the infections. On the cell wall surface $C$. albicans exposes ligands of receptors of human cells, and other molecules which are responsible for adhesion to epithelial and endothelial cells, plastic, cell-cell aggregatoin and formation of biofilm (Chaffin, LopezRibot et al. 1998). Moreover, the hydrophobicity of the cell wall surface also mediates the process of adhesion (Verstrepen and Klis 2006).

In an attempt to explore the adhesion defects of phrls mutant at a molecular level, we analyzed the expression of a few marker genes encoding GPI- 
proteins. $H W P 1$ is a gene encoding a highly mannosylated surface protein that also acts as a target of mammalian transglutaminases and generates covalent cross-links involved in adhesion to human cells. It is also a marker of hypha-specific expression and is also required for hyphal development and virulence (Sharkey, McNemar et al. 1999; Tsuchimori, Sharkey et al. 2000). $E C E 1$, which shares a partial common regulation at a transcriptional level with HWP1 (Sharkey, McNemar et al. 1999) encodes a cell surface protein. ECE1 is up-regulated as a function of the hypha elongation but it is dispensable for hyphal development (Birse, Irwin et al. 1993). Interestingly, we detected a reduction of 60 and $65 \%$ respectively in the expression levels of of $H W P 1$ and ECEl in the PHRI deleted strain at $2 \mathrm{~h}$ from induction of hyphal growth in adhesion to plastic. This suggests that negative morphogenically regulated pathways are activated by the inability to elongate the germ tubes in the mutant. CaNrg1 or Tup1 could be transcriptional repressors involved in mediating such a modulation but we cannot exclude other mechanisms of regulation. ALS3 is an important adhesion-encoding gene of $C$. albicans. It belongs to Als family and is a mannoprotein of the outer flocculant layer which was detected along the entire surface of the germ tubes (Coleman, Oh et al. 2009). It is involved in cell aggregation and adhesion to epithelial and endothelial cells probably (Oh, Cheng et al. 2005; Chaffin 2008). We did not find differences in the transcript level of $A L S 3$ in the PHR1 null mutant compared to the isogenic strain. This suggests that $A L S 3$ is induced in the frame of the commitment to the hyphal genetic program of the mutant but unlike $H W P 1$ and ECE1, which are also hyphae specific genes, it is not repressed. This suggests that either ALS3 has a different kinetic of repression or is not a target of the morphogenically repression pathway. However, the defects in cell wall assembly due to the lack of Phrlp could affect the localization of the $A L S 3$ gene product or the different architecture of the phr1 cell wall could shed the protein and reduce its interaction with the plastic surface. It has been recently shown that an anti-Als3 monoclonal antibody can block the adhesion of C.albicans suggesting that exposure of the protein epitopes is crucial or the adhesion process (Coleman, Oh et al. 2009). ALS3 expression could explain the residual capability of the mutant to adhere. Similar comments can be made for EAP1. We cannot exclude that also EAP1 gene product is not properly localized at the surface and this could 
affect adhesion in the mutant. Phrlp, through its activity on cell wall glucan, creates the attachment site for other mannoproteins (Fonzi 1999). In the absence of Phrlp activity the proper anchoring and specific localization of surface mannoproteins may be altered as mannoproteins are diverted to the binding with chitin. As a consequence the molecular mechanism of adhesion could be affected.

It should be recalled that Phr1p was also identified as a component of the cell wall proteome (de Groot, de Boer et al. 2004) and can be detected as extractable cell wall protein (Urban, Sohn et al. 2003). This suggests that Phrlp could be anchored to the plasma membrane through GPI but a fraction, could undergo a transglycosylase reaction at the cell surface and be cross-linking to the glucan network, as occur for other cell wall mannoproteins (De Groot, Ram et al. 2005). Phr1p could be exposed to the cell surface and remodel the outer surface layer also affecting the position of cell wall mannoproteins. Further studies are necessary to determine the localization of Phr1p and its role in remodeling the cell wall surface during adhesion.

Using a reconstituted human epithelial model (RHE), in which a confluent monolayer of the enterocytic cell line
Caco2 was used, we have found that the deletion of PHRI abolishes the ability of penetrate into the tissue and causes attenuated epithelial damage. The reduced adherence of the phrls mutant can influence in the ability of the mutant to invade epithelia as attachment to the epithelial cells is considered to be the initial step in the infection process followed by other processed such as the formation of epithelial cell protrusions and membrane ruffling characteristic of induced endocytosis or active penetration of C. albicans in the epithelia. Interestingly, in a recent study in which a molecular dissection of the distinct stages of infections as well as transcriptional profiles during infection of human epithelial model (RHE) have been performed, PHRI, together with PRAI and RIM101 have been found to be induced during the invasion phase (Wilson, Thewes et al. 2009). Upregulation of these genes gives emphasis to the importance of the $\mathrm{pH}$ response in the infection process. Upregulation of PHRI is particularly interesting since this data is in agreement with our findings of the strongly reduced ability of phrls mutant to invade and cause tissue damage.

The combined defects in adhesion and invasion we reported here are likely to contribute to the avirulence of the 
mutant reported in a mouse model of systemic infection (De Bernardis, Muhlschlegel et al. 1998). Consistently with a crucial role of PHRI in the attachment to and invasion of epithelia, a recent large scale analysis of the $C$. albicans transcriptome highlighted the occurrence of PHRI transcript among the most induced gene during progression of $C$. albicans infections of human oral epithelial cells (Wilson, Thewes et al. 2009).

Taken together, our findings highlight the importance of the Phr1 protein in the pathogenesis of Candida albicans. Since it is known that alterations in the cell wall introduce variations at the $C$. albicans surface that could have implications in the interaction of the fungus with the host, our data should be interpreted on the basis of an that alteration of the cell wall as a consequence of the lack of the cell wall remodelling activity of the Phr1 protein. With this work we have gain insights into the molecular basis of the reduced virulence of Phr1 protein and highlight the potential of the protein as a good molecular target for the development of new antifungal drugs. Nevertheless more efforts are necessary to understand how the cell responds to the alterations of the biochemical and physical properties of the cell wall as a consequence of the lack of the Phr1 protein. 


\section{CHARACTERIZATION OF THE phr1A MUTANT PHENOTYPE DURING FILAMENTATION ON SOLID MEDIUM:}

The phenotype of phrls mutant during filamentation has been extensively described (see pages 36-27 of the Introductión). As shown in Figure 6, at alkaline $\mathrm{pH}$ phrld mutant is unable to sustain apical growth. The phenotype of the mutant during growth in solid media has not yet characterized. In this work we have demonstrated that the mutant is completely unable to form hyphae on solid Medium 199 at pH 7.5 or 8 (Figure 7).

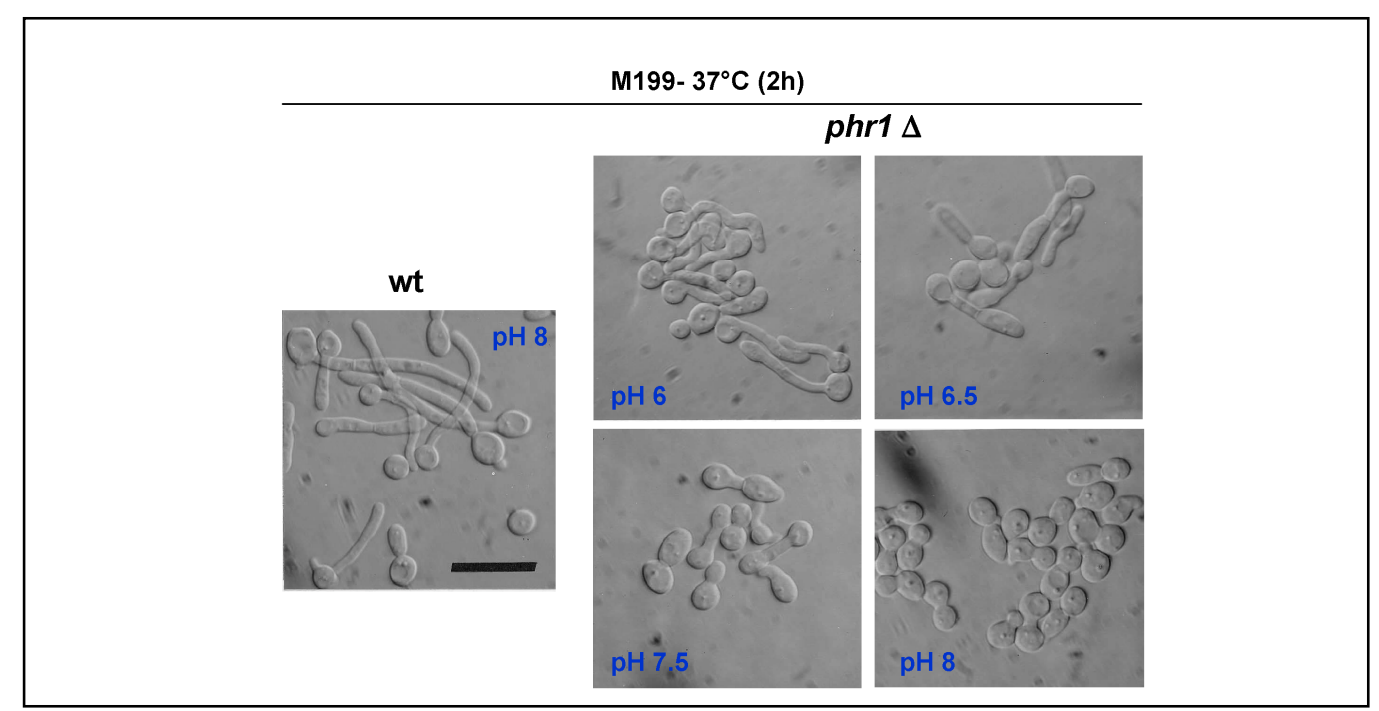

Figure 6. Phenotype of phrl $1 \Delta$ mutant on liquid media at different values of $\mathrm{pH}$ (from Saporito-Irwin, Birse et al. 1995)

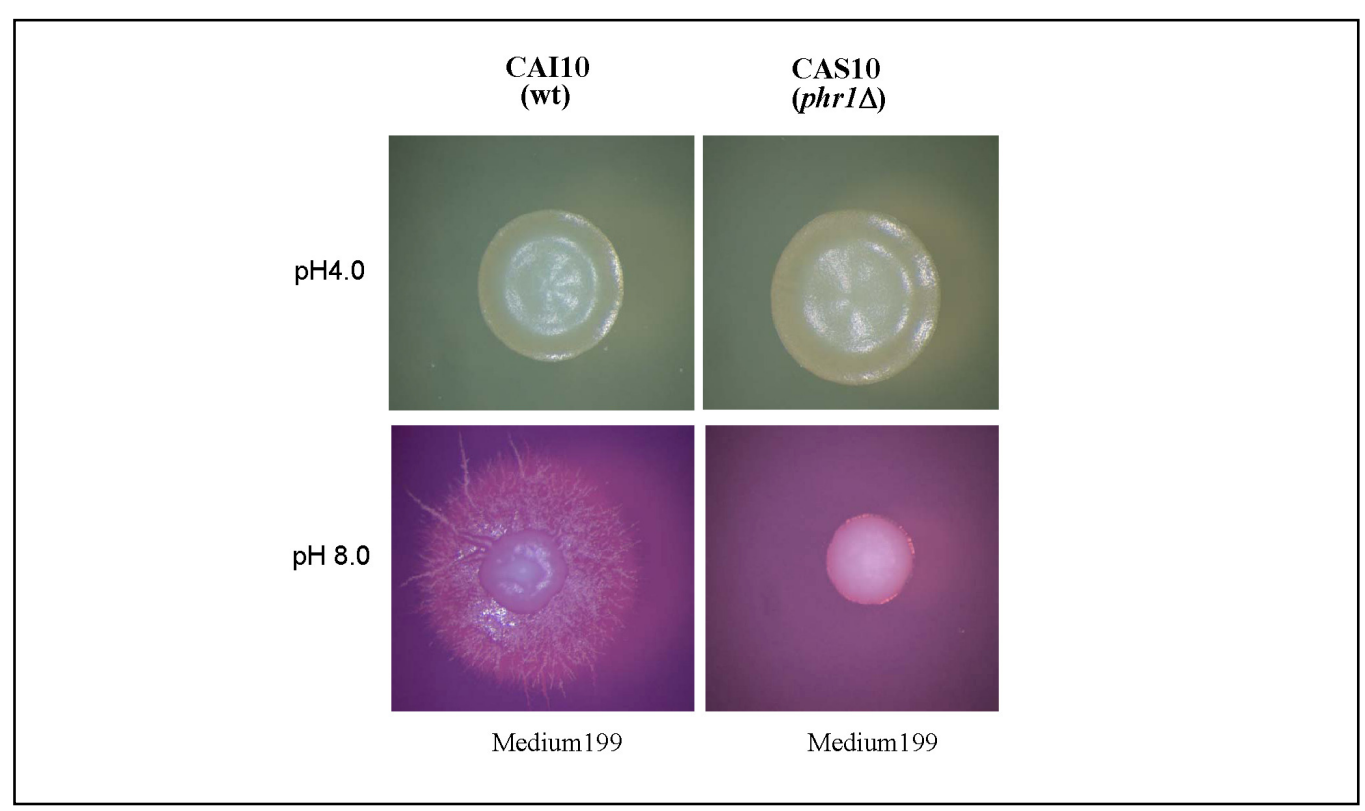

Figure 7. Phenotype of phrls mutant on solid media at $\mathrm{pH}$. The induction of the filamentous growth on solid media was performed as described in Materials and Methods. 


\section{IMMOBILIZATION OF}

Gas1p OF S. cerevisiae IN THE CHITIN RING IS REQUIRED FOR PROPER

\section{MORPHOGENESIS:}

\section{BACKGROUND:}

The components of the yeast cell wall are linked to each other as macromolecular complexes in which a $\beta$-(1,6)-glucan acts as a cross-linker, being attached to the major components, $\beta$-(1,3)-glucan and mannoproteins, and occasionally to chitin (Kollar, Reinhold et al. 1997). Polymer cross-linking contributes to the mechanical strength of the cell wall but it is also important in the loosening and remodelling of the cell wall required during morphogenetic processes, such as bud emergence (Cabib, Roh et al. 2001).

This organized structure of crosslinked macromolecules results from the coordinated actions of several extracellular enzymes, most of which have still to be characterized, and from the integration of these extracellular assembly processes with the cytoskeleton and polarity machinery of the cell (Firon, Lesage et al. 2004). Gas protein family members are well known cell wall remodelling enzymes (Popolo and Vai 1999; Mouyna, Fontaine et al.
2000; Carotti, Ragni et al. 2004). GAS gene family of Saccharomyces cerevisiae is composed of five members (GAS1-5). As mentioned in the Introduction Gas1p is the best characterized member of the Gas protein family. This protein is crucial for proper cell wall assembly and morphogenesis. In fact, mutants deleted in the GAS1 gene in Saccharomyces cerevisiae display morphological defects, including a rounder shape and larger size and clumpy phenotype and exhibit a reduced growth rate (Popolo, Vai et al. 1993). Gas $1 \Delta$ cells prevent lysis, caused by cell wall weakening, by activating a compensatory response involving the upregulation of several genes and changes in the composition and architecture of the cell wall (Popolo, Vai et al. 1993; Lagorce, Hauser et al. 2003). GAS1 is expressed during vegetative growth together with GAS5, which appears to play an auxiliary role, and GAS3, which is expressed at a very low level and whose function is still unknown.

A common feature of all Gas proteins is the attachment of a glycolipid moiety, a glycosylphosphatidylinositol (GPI) to the C-terminal region of the protein that anchors the protein to the outer leaflet of the plasma membrane. GPI confers lateral mobility in to the lipid environment to linked proteins 
(Malinska, Malinsky et al. 2004) and promotes association with specific membrane microdomains, which have also been named "lipid rafts", enriched in sphingolipids and ergosterol. The tight packing organization and composition of these membrane microdomains makes them insoluble in non-ionic detergents, such as Triton X-100 (TX100) at low temperatures (Bagnat, Keranen et al. 2000). Gas1p was the first recognized marker protein recovered in the detergent-resistant membranes (DMRs), also named detergent-insoluble glycolipid-enriched complexes (DIGs) (Bagnat, Keranen et al. 2000). Recently, Gas1p, Gas3p and Gas5p were all found to be enriched specifically in the plasma membrane derived DRMs (Aronova, Wedaman et al. 2007).

Gas1p has been considered for many years as a canonical plasma membrane protein, but a possible cell wall anchorage of the protein was reported by De Sampaio and col. in 1999 when they showed that the protein was released from the cell wall by $\beta$-1,3-treatment (De Sampaio, Bourdineaud et al. 1999). Moreover, mass spectrometry analyses of the cell wall proteome have revealed that Gas1, Gas3 and Gas5 proteins are covalently cross-linked to the glycan network (Yin, de Groot et al. 2005). Together with Gas proteins, other GPI- mannoproteins with putative cell wall cross-linking activities were recovered in the cell wall proteome. Among them, the GPI-proteins Crh1p and Crh2p, which are putative transglycosydases required for the linking of the chitin to $\beta-(1,3)$ glucose branches of the $\beta$ - $(1,6)$-glucan in the cell wall. This activity is high in the bud scars consistent with the localization to these proteins (Yin, de Groot et al. 2005; Cabib, Blanco et al. 2007; Cabib, Farkas et al. 2008).

\section{OBJECTIVES:}

The aim of this work was to study the localization of Gas1p by exploiting fluorescent protein fusion techniques. We wanted to analyze the localization of the protein during vegetative growth and in conditions of highly polarized growth. In addition, the aim of this study was to explore whether the cell wall anchoring of Gas1p was due to a leakiness of the mechanism of retention in the plasma membrane or a specific destination of the protein 
RESULTS:

\section{Construction of a functional version of mRFP-Gas1 fusion protein:}

Since Gas1 protein contains a signal peptide at the $\mathrm{N}$-terminal that is required for its translocation in the secretory pathway and a C-terminal sequence necessary for the attachment of the GPI, an internal tagging was necessary for the study of the localization of the protein in living cells. For the internal tagging we used a construct of a monomeric Red Fluorescent Protein (mRFP) that was reported by Fujita et al. in a successfully tagging of Gas1p for a different purpose (Fujita, Yoko et al. 2006). We used the integrative plasmid pMF608, which was kindly donated by the authors and harboured, an in-frame fusion of the signal peptide, the mRFP coding sequence and GASI orf. The creation of the fusion gene is described in Materials and Methods. Figure 1A shows a schematic representation of the fusion protein. AN117-16D and AN117-4B strains were transformed with the linearized plasmid but in both cases a weak signal fluorescent was obtained. Diploid strains obtained by crossing both AN117-16D and AN117-4B with ER311 which harbours a GAS1 deletion gave also a weak red fluorescence. By using another strain, W3031A, with a different genetic background we were able to obtain a bright red fluorescence but most of the the fluorescence in the cytosol of the cells in a perinuclear compartment which was indicative of a localization in the ER (data not shown). Interestingly, we found that mRFP-Gas1p was properly produced and transported to the cell surface only in a gas 1 null mutants (WAH strain) suggesting that that the co-expression of the fusion protein competes with the endogenous Gas $1 p$ for folding and that the retention of the hybrid protein in the ER was probably the reason the presence of perinuclear fluorescence in wild type cells (data not shown). Different gas 1 clones expressing mRFP-Gas1p were analyzed with which we could preliminary detect a clear labelling at the cell surface in agreement with a plasma membrane localization of Gas1p. Finally, clone 9 was used for further studies and the transformant strain expressing the mRFP-Gas1 fusion protein was named JC9.

In order to determine if the fluorescent fusion protein was functional we tested its sensitivity to Calcofluor White $(\mathrm{CW})$, a cell wall perturbant agent. This dye binds to nascent chitin fibrils and destabilizes cell wall. Cell wall mutants, such as $\operatorname{gas} 1 \Delta$, are hypersensitive to $\mathrm{CW}$ because of their compensatory chitin hyperpaccumulation (Popolo and Vai 1999; Valdivieso, Ferrario et al. 2000). 
In this test gas $1 \Delta$ was used as a control to test the ability of the strain expressing the fusion protein (JC9) to suppress $\mathrm{CW}$ hypersensitivity. As shown in Figure 1B, mRFP-Gas1 fully complemented the CW-hypersensitivity of gas $1 \Delta$ cells indicating that the hybrid protein was functional. As shown in the microscopy images of Figure 1C, mRFP-Gas1p also rescued other phenotypic traits typical of gas $1 \Delta$ cells, such as the round morphology, the larger size and the appearance of "mickey mouse" cells and clumpy phenotype (the last two traits are a consequence of defective bud maturation and cell separation) (Popolo, 1993).

al.

et

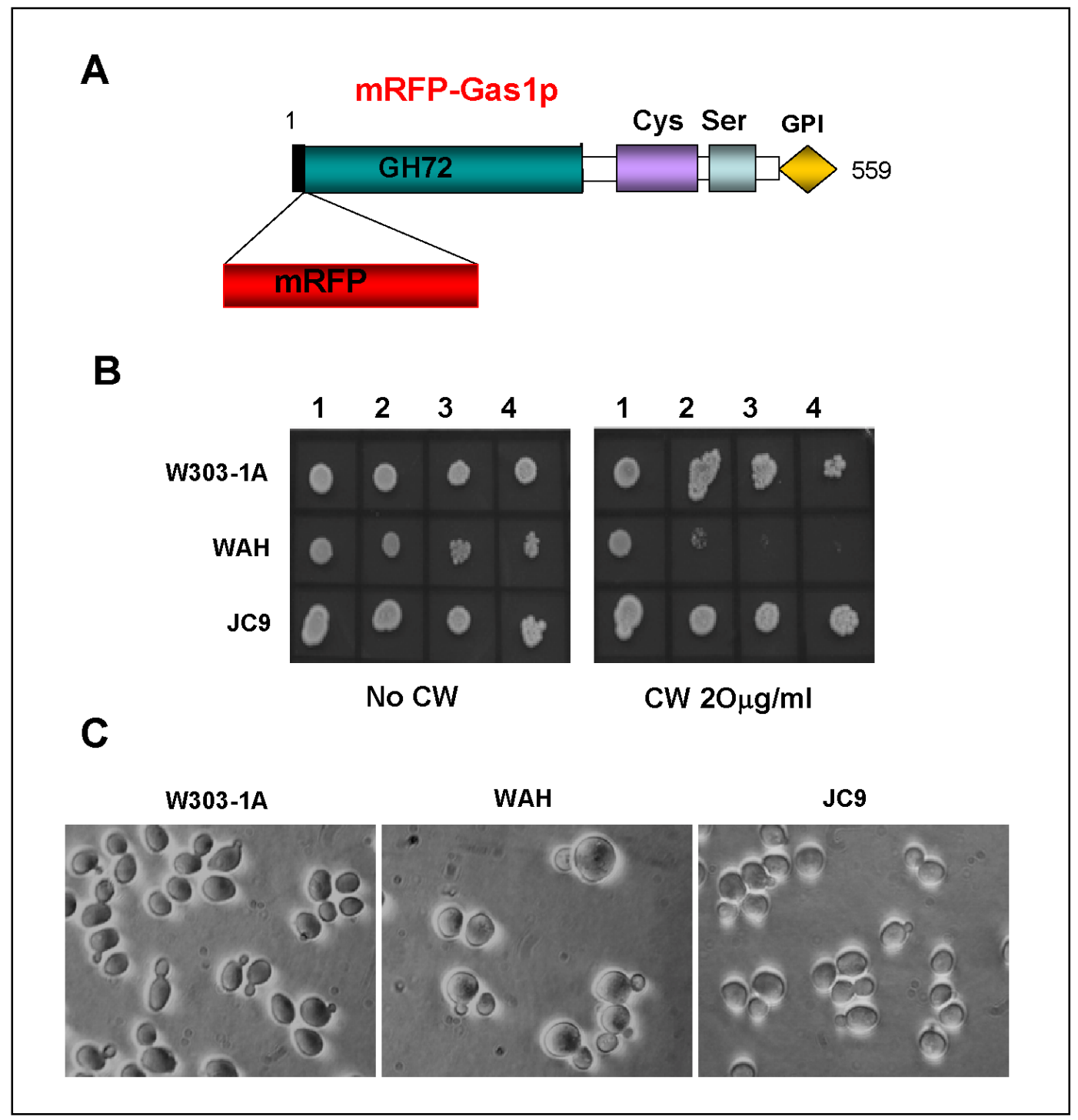

Figure 1. Fusion of mRFP at the N-terminal region of Gas $1 p$ results in a functional protein. A. Schematic representation of the domain organization and the site of insertion of mRFP into the GAS1 gene. B. Calcofluor White $(\mathrm{CW})$ sensitivity was assayed by drop test on YPD plates containing $20 \mu \mathrm{g} / \mathrm{ml}$ of $\mathrm{CW}$. C. Micrographs of formaldehyde-fixed cells growing in YPD at $25^{\circ} \mathrm{C}$. 
Moreover, the reduced growth rate $(\mu)$ of gas $1 \Delta$ cells, $0.254 \pm 0.02 . \mathrm{h}^{-1}$, was reverted to the value of the parental strain, $0.66 \pm 0.05 \mathrm{~h}^{-1}$, being the value of the growth rate of the strain expressing mRFP-Gas1p $0.65 \pm 0.04 \mathrm{~h}^{-1}$. The growth rates of the parental strain (W303), the gas1s strain (WAH) and the strain expressing mRFP-Gas1p (JC9) were calculated with three independent experiments in which the growth was monitored as described in Materials and Methods.

Gas1p is localized at the cell periphery, at the mother-daughter neck region, and in the bud scars:

Microscopy observations of exponentally growing cells allowed us to determine that mRFP-Gas1p is present at different locations. Cells were grown at $30^{\circ} \mathrm{C}$ in YPD and harvested for microscopy analysis at different time points. Percentage of budding was calculated by counting at least 200 cells after mild sonication. With fluorescent microscopy analysis we found that mRFP-Gas1p decorates the cell periphery consistently with the plasma membrane localization of the protein (Figure 2a). In medium-budded cells two bright dots appears at both sides of the neck region between the mother and daughter cells (Figure 2b) and in largebudded cells the red fluorescence nicely decorates the entire septum region (Figure 2c). Interestingly, in some cells we could observed bright crater-like regions (arrow in Figure 2d) and also some crescent-shaped fluorescent segments (head arrows in Figure 2d) that may represents lateral views of one of more adjacent bud scars.

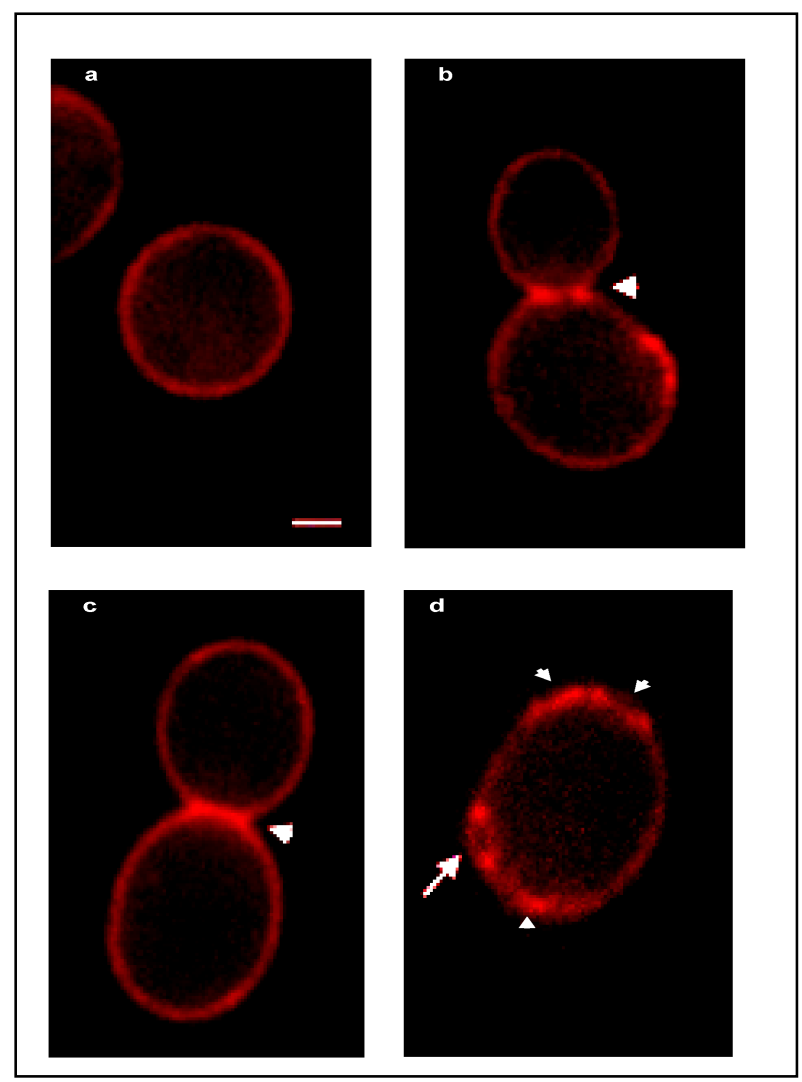

Figure 2. Confocal microscopy images form cells from strain JC9, a gas $1 \Delta$ strain expressing mRFP-Gas1p, growing in YPD at $30^{\circ} \mathrm{C}$. Bar, $1 \mu \mathrm{m}$. 
Similar results of the localization of Gas1 during vegetative growth were obtained by using a fusion with the Green Fluorescent Protein (GFP). (Eleonora Rolli's PhD Thesis and Rolli, Ragni et al., 2009).

In this case, the site selected for the insertion was a Ser-rich region before the GPI-anchored sequence at the Cterminal region of the protein. In this case, the fusion gene was not integrated into the genome but was placed on episomic plamids. When this proteins was expressed both in a gas $1 \Delta$ mutant strain and in the parental strain with a different genetic background with respect to the fusion with the Red Fluorescent Protein, it was found be located also in the four locations that I mentioned before. It was present in the plasma membrane, in the motherdaughter neck in the septum and in bud scars. Together, these results indicated that independently of the type of fluorescent protein, site of fusion, genetic background or gene dosage of the construct Gas1p presents the same localization. Figure 3 shows an example of the localization of Gas1p-GFP in yeast cells.

By use of the two different fusions we could conclude that Gas1p is present at the cell periphery, in the bud neck region in small-budded cells, in the septum region in large-budded cells and in the bud scars where it remains for several generations.

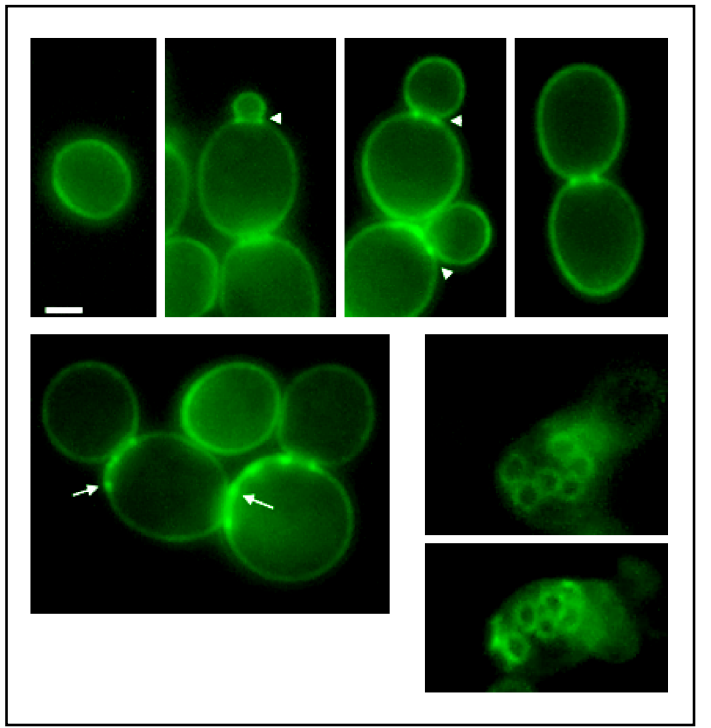

Figure 3. Cells of the diploid strain ER336 (a diploid gas $1 \Delta$ mutant carrying plasmid pRS416GAS1-GFP) were grown to log phase in YPD at $30^{\circ} \mathrm{C}$. Cells were observed in PBS without fixation. The figure shows the various subcellular locations of Gas1p. Arrowheads: two dots aside the neck or septum. Arrows: bud scars. Bar, $3 \mu \mathrm{m}$.

\section{mRFP-Gas1 fusion protein localizes in} mobile and immobile pools:

In order to investigate whether Gas1p localization changes in conditions of high polarized growth, we designed an experiment with the addition of $\alpha$-Factor $(\alpha-F)$, a situation that arrests cells in G1. The experiments were performed with cells expressing the mRFP-Gas1 fusion protein (MATa cells) that were treated with $\alpha$-Factor. The effect on cell cycle 
progression was monitored by measuring the decrease of budding index (Figure 4A) and the appearance of shmoos. Microscopy analysis revealed that addition of $\alpha$-Factor to the culture induced a rapidly decrease of the mRFPGas1p fluorescence around the cell.

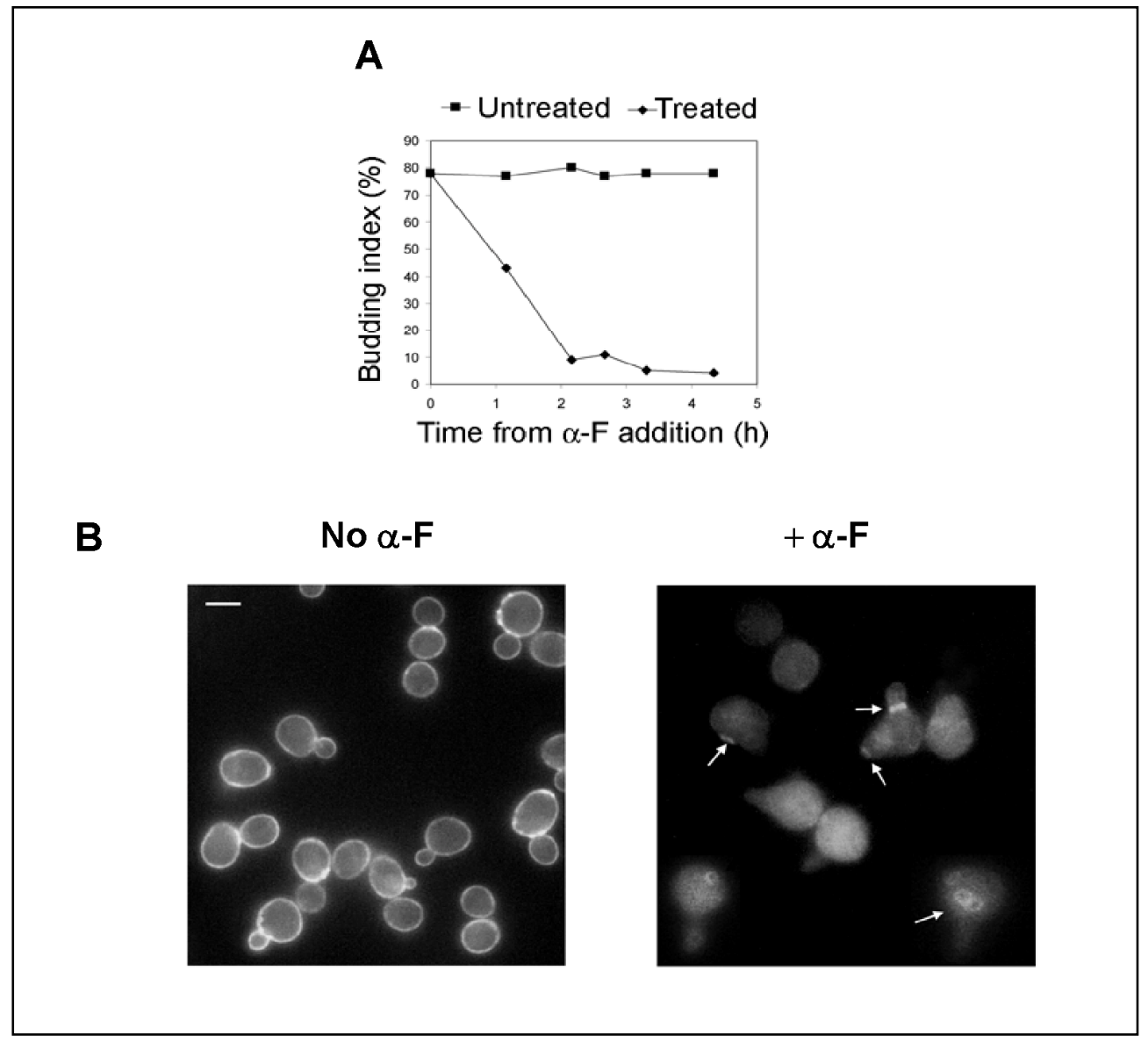

Figure 4. Cells from strain JC9 treated with $\alpha$-Factor. Cells from an exponentially growing culture were treated with $20 \mathrm{mg} / \mathrm{ml}$ of $\alpha$-Factor. A. Plot showing the percentage of budded cells in W303-1A or JC9 cells. B. Microscopy analyses of cells expressing mRFP-Gas1p. Cells were collected, fixed with formaldehyde and washed twice with PBS. Left panel shows the cells time zero (right before $\alpha$-Factor addition). Right panel shows the same cells after 3.25h from $\alpha$-Factor addition.

We observed that the signal on the cell surface became more and more weak with the time course of the experiment and after $3.25 \mathrm{~h}$ from the addition of $\alpha-F$, the red fluorescence signal at the cell contour was almost undetectable except for bud scars and rare septa that were still fluorescent (Figure 4B).

These findings suggested that mRFPGas1p in the membrane could be labile and the fraction of the protein that is cross-linked to the bud scars could be protected from degradation. So, we moved from our initial purpose to 
investigate the polarized localization of the protein in the shmoos to thoroughly study these new findings. To further test our hypothesis we determined the level of the fusion protein at different time points after addition of $\alpha$-Factor and compared it with the level of Gas1p in the wild type strain. Immunoblot of total protein extracts of both the wild type and the strain expressing the fusion protein was performed and, as shown in Figure $5 \mathrm{~A}$, Gas1p was detected as a polypeptide of about $125 \mathrm{kDa}$ whereas mRFP-Gas1p migrated as a polypeptide of about 155 $\mathrm{kDa}$, which is compatible with the molecular weigh expected for Gas1p fused to mRFP ( 30 kDa). During the preparation of the extracts the cell walls were discarded and so Gas1p possibly cross-linked to them was not recovered.

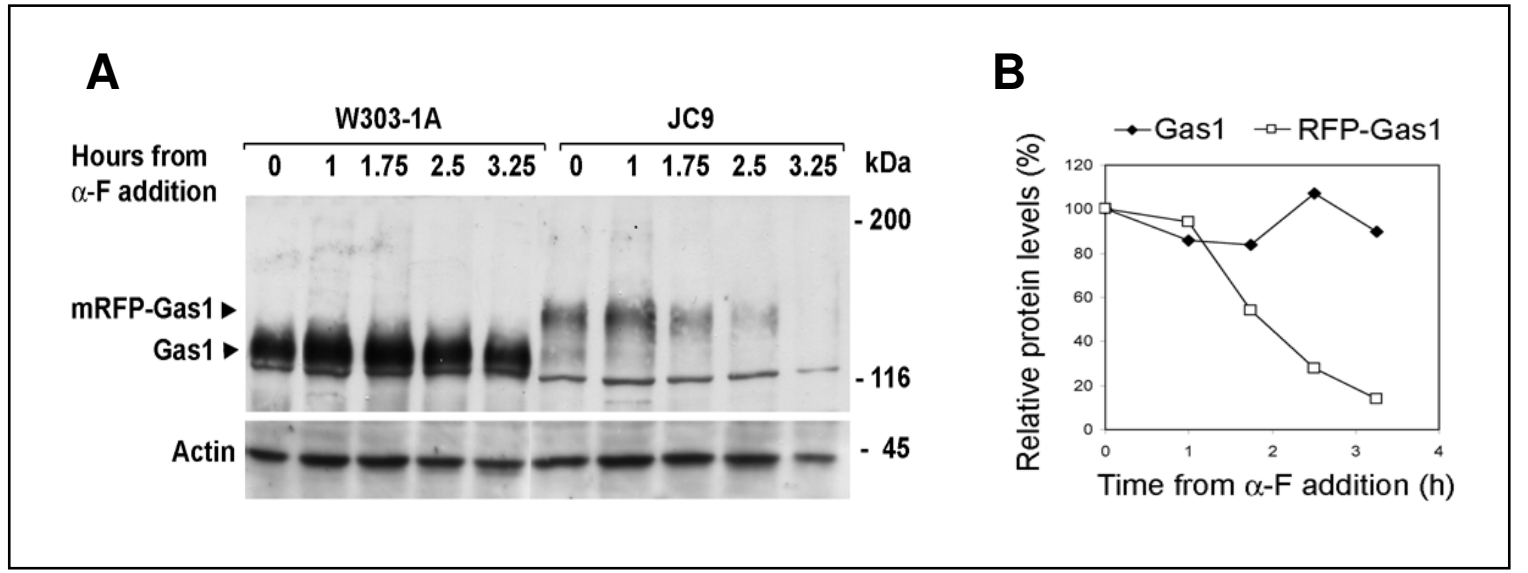

Figure 5. A. Immunoblot analysis of total protein extracts (about $80 \mu \mathrm{g}$ protein) from W303-1A and JC9 cells at different time points from $\alpha$-Factor addition. B. Relative protein levels of Gas $1 \mathrm{p}$ and mRFP-Gas $1 \mathrm{p}$ determined by densitometric analysis of the bands of the immunoblot in $\mathrm{B}$ as a function of the time of treatment with $\alpha$-Factor.

With the addition to $\alpha-F$, the intensity of the 125 kDa-band did not significantly change even after a long exposition to the pheromone. Even at $3.25 \mathrm{~h}$ after addition of $\alpha-\mathrm{F}$, the level of the protein was mainly the same as it was at the beginning. By contrast, the 155 kDa-band appeared to rapidly diminish with a significantly decrease in its level after $1.75 \mathrm{~h}$ and became undetectable after $3.25 \mathrm{~h}$ of treatment. Levels of Gas1p and mRFP-Gas1p polypeptides were normalized to the intensity of the actin band. As shown, in Figure 5B, the level of the endogenous Gas1p maintained approximately constant whereas mRFP-Gas1p levels strongly decreased during the treatment with $\alpha-F$. Moreover, the levels measured at time zero indicated that the 
steady-state level of mRFP-Gas1p was $50 \%$ the level of Gas1p. Together these results point out that the fusion protein is either less synthesized or becomes degraded more rapidly than the endogenous protein during vegetative growth.

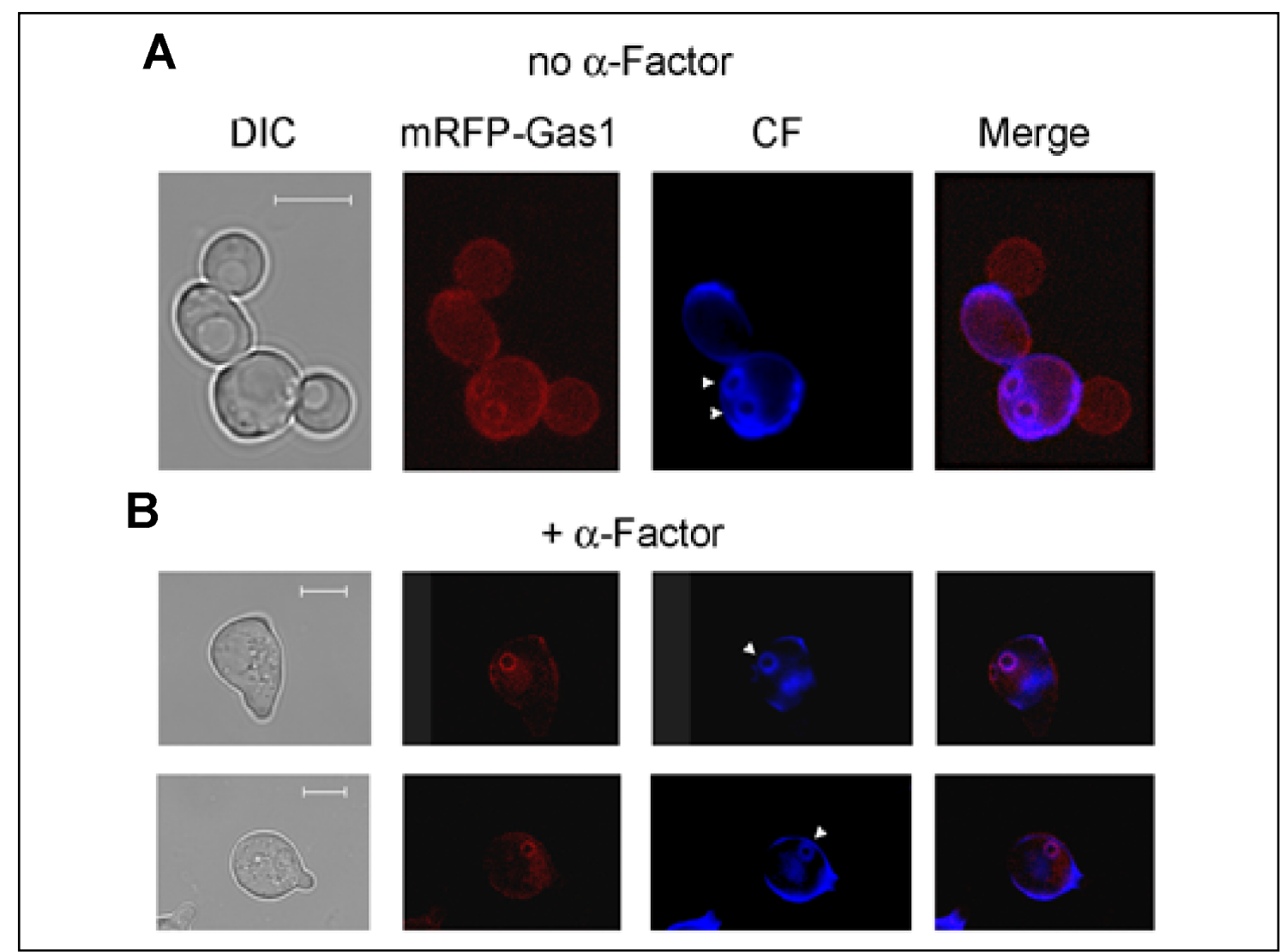

Figure 6. Confocal microscopy images from cells carrying the mRFP-Gas1 construct growing to log phase in YPD at $30^{\circ} \mathrm{C}$ and treated with $\alpha$-Factor. The images are the result of superimposed optical sections. A. Cells collected right before the addition of $\alpha$-Factor . B. Cells collected 3.25h after $\alpha-\mathrm{F}$ addition.

Since GAS1 expression is turned off and its mRNA levels rapidly decrease during $\alpha-F$ treatment (Popolo, Cavadini et al. 1993), these results suggest an increase in the rate of degradation of mRFP-Gas $1 p$ compared to Gas1p. Thus, the rapid disappearance of mRFP-Gas $1 p$ from the cell contour observed by microscopy analysis is presumably due both to a high turnover of the protein, which causes a removal of the protein from the plasma membrane, and to the absence of de novo synthesis.

We exploited the instability of mRFPGas1p to analyze the localization of the fluorescence labelling that persisted after $\alpha-F$ treatment. Confocal microscopy analyses were performed using $\mathrm{CW}$ to 
stain chitin in cells expressing the mRFP-Gas1 protein. For chitin staining CW was added directly to the culture as described in Materials and Methods.

During vegetative growth mRFPGas1p decorates the cell surface and, as shown in Figure 6 (upper panels), clearly colocalizes with the bud scars (arrows in Figure $6 \mathrm{~A}$ ) as detected by the merging of the red and blue fluorescence which produces a light pink color. After 3.25htreatment with $\alpha-\mathrm{F}$, the red fluorescence at the cell surface strongly decreased but the signal from the merging in the bud scars remained intense (Figure 6, lower panels).

Together these results indicate that Gas1 protein fused to mRFP produces a protein that is more unstable than the endogenous Gas1p and undergoes a high turnover in the plasma membrane whereas it is stabilized in the bud scars.

\section{The immobile form of Gas1 is cross-}

\section{linked to the bud scars:}

The particular behaviour of the fusion of mRFP at the N-terminal end of Gas 1 protein described above allowed us to further investigate the localization of Gas1p in the bud scars. Subcellular fractionation was performed in order to determine whether mRFP-Gas $1 p$ in $\alpha-F$ treated cells was covalently bound to the bud scars. Based on the results regarding the presence of mRFP-Gaslp in two fractions, a mobile fraction localized in the plasma membrane and an immobile one which is likely to be bound to the cell wall, we hypothesized that in cells treated with $\alpha-F$ we should not detect to the fraction in the membrane but we should still detect mRFP-Gas1p bound to the cell wall fraction.

Western blots experiments were performed from subcellular fractions and purified cell walls that were obtained at time zero and after $3.25 \mathrm{~h}$ after $\alpha$-Factor addition according to the scheme shown in Figure 7A. Proteins were analyzed by immunoblot using anti-Gas1p antibodies. As shown in Figure 7B, both Gas1p and mRFP-Gas1p were detected in the membrane fraction from untreated cells. After $3.25 \mathrm{~h}$ of treatment with $\alpha-\mathrm{F}$, Gas1p was enriched in the membrane fraction whereas mRFP-Gas1p was not detectable in agreement with the lability of the fusion protein. In addition, the proteins were also detected in the material released after treatment of purified cell walls with $\beta$-1,3-glucanase in agreement with the presence of Gas $1 p$ in the cell wall proteome (De Sampaio, Bourdineaud et al. 1999; Yin, de Groot et al. 2005). 

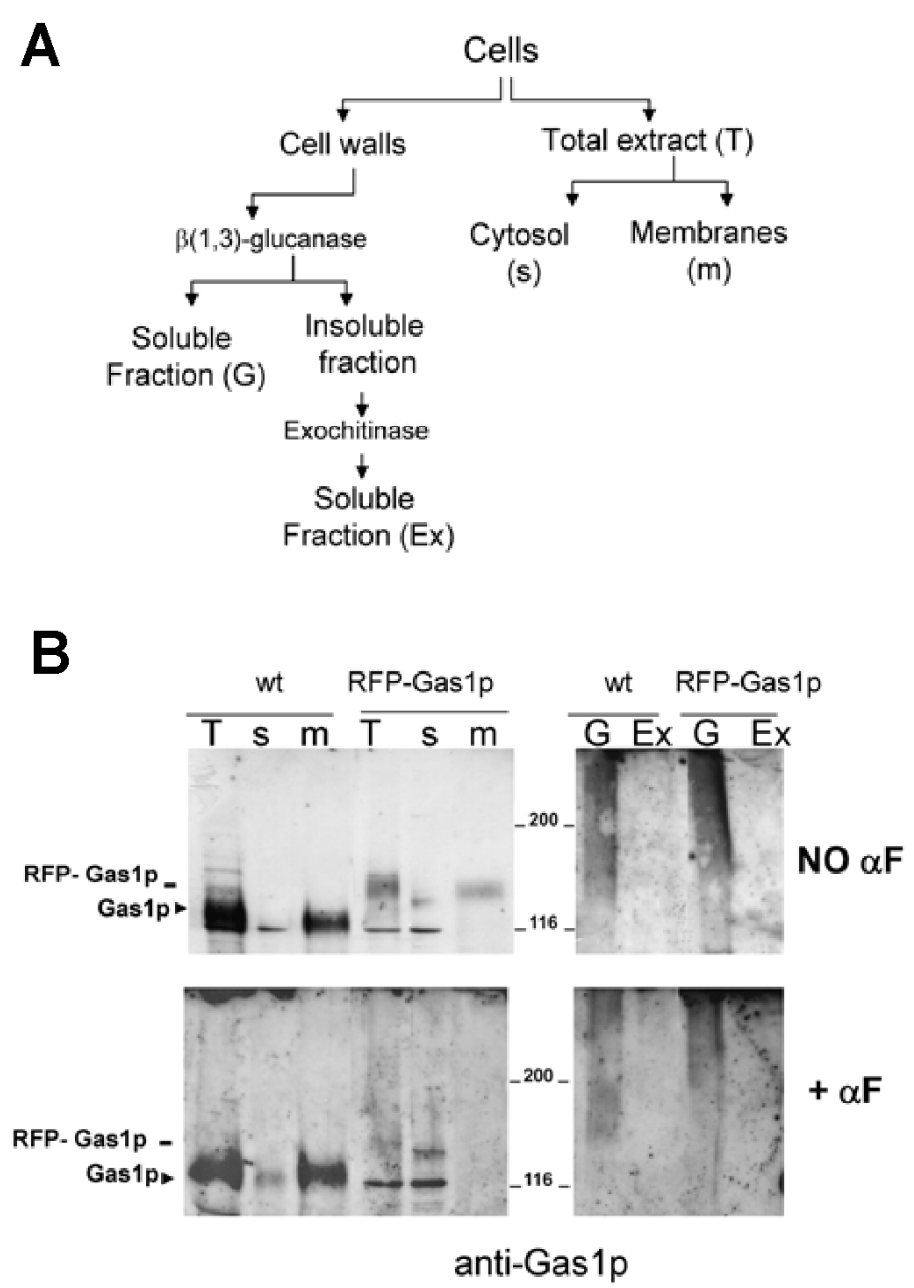

Figure 7. Subcellular fractionation and cell wall analysis of $\alpha$-Factor treated cells. A. Flow chart of subcellular fractionation and cell wall treatments. B. Left: immunublot analysis of total protein extracts $(\mathrm{T})$, soluble $(\mathrm{S})$ and membrane $(\mathrm{M})$ fractions form W303-1A and JC9 cells before and 3.25h from the addition of $\alpha-F$. Right: b- $(1,3)$-glucanase extract $(\mathrm{G})$ of isolated cell walls and exochitinase extracts (E) from the residual insoluble fraction.

The electrophoretic mobility of the cell-wall forms of Gas1p and RFPGas1p is rather heterogeneous in size (>130 KDa) consistent with the proteins that are covalently linked to glucans of different size and depending on accessibility of the glucan chains to the enzymatic cleveage. It is known that a minor fraction of GPI-CWPs binds to chitin through a short $\beta$-(1,3)-glucan side branch of the $\beta$-(1,6)-glucan (Kollar, Reinhold et al. 1997). If Gas1p were linked to chitin through this alternative linkage should become soluble upon action of exochitinase (Kollar, Reinhold et al. 1997). So, we treated the purified 
cell walls with exochitinase. The commercial preparation contained a protease that degraded Gas1p so we used pure exochitinase in this study. The treatment of the cell walls released both Gas1p and RFP-Gas1p (Figure 7B) indicating that these protein are also linked to chitin probably trough a $\beta$ $(1,6)$-glucan. After $\alpha-F$ treatment the enzymatic treatment did not release Gas1p or RFP-Gas1p at a detectable level. After 3, $25 \mathrm{~h}$ of treatment with $\alpha$ $\mathrm{F}$, Gas1p was enriched in the membrane fraction whereas mRFP-Gas $1 p$ was not detectable. On the other hand in the $\beta$ $(1,3)$-glucanase-release fraction from purified cell walls both Gas1p and mRFP-Gas1p were still present (Figure 7B). Therefore, two cell-wall bound pools of Gas1p exist: one is linked to $\beta$ $(1,3)$-glucan and was previously described, the other is directly bound to chitin and was never described before. Interestingly this second form is not recovered after $\alpha-f$ treatment suggesting that its synthesis probably ceases when cells arrest in G1 phase and that the previous form could bee converted in a new complex which is not solubilized by exochitinase.

\section{Loss of GAS1 affects the width of the} neck region:

In order to explore the role of Gas 1 protein localization in the chitin ring we investigated whether the lack of Gas1p could affect the morphogenesis of the neck region. The neck region is constriction that is formed between the mother and daughter cells in the moment of bud emergence. Normally the width of the neck region remains constant through the cell cycle since growth in that area is prevented (Rodriguez-Pena, Rodriguez et al. 2002).

The width of the neck region was measured in gasl null mutant cells (WB2d) and in wild type cells (W3031A). As shown in Figure 8, the width of the neck region increases almost 2 times its diameter. The enlargement of the neck suggests that Gas1p in the chitin ring could be necessary to maintain the rigidity required in the neck region. 


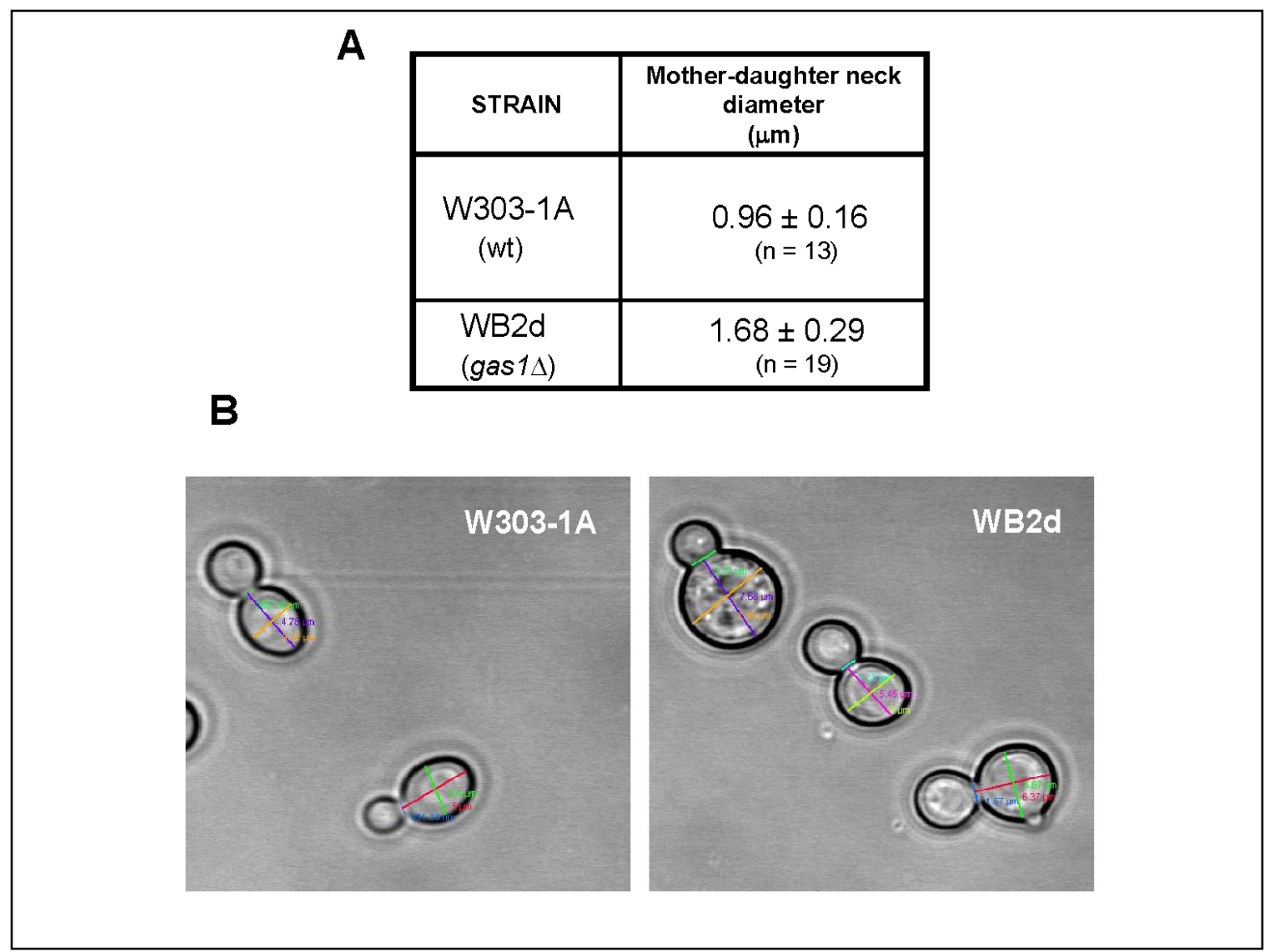

Figure 8. Enlargement of the mother-daughter neck in gas $1 \Delta$ mutant cells. A. mean values $( \pm$ s.d.) of the measurement of the diameter $(\mu \mathrm{m})$ of the neck region. B. Examples of the micrographs obtained at the confocal microscope where the measurements of the wideness of the neck where performed. 


\section{DISCUSSION:}

Despite large scale approaches of random fusion or targeted fusion of GFP into all yeast ORFs were published a few years ago (Huh, Falvo et al. 2003), the localization of GPI-anchored proteins was not be reliably established. Moreover, the fusion of the fluorescent protein at the $\mathrm{C}$-terminal end before the STOP codon caused the loss of the GFP as GPI-proteins have a C-terminal signal sequence for GPI attachment that is removed during the processing of the protein in the ER. In this study, we have investigated the localization of Gas $1 p$ by exploiting fluorescent protein fusion techniques and our current knowledge of the domain organization of Gas1p. An $\mathrm{N}$-terminal mRFP-Gas1 fusion protein, where mRFP was inserted immediately after the $\mathrm{N}$-terminal signal peptide, was expressed from its endogenous promoter in a gas $1 \Delta$ mutant derived from the haploid strain W303-1A. The construct was integrated into the yeast genome and directed the expression of a functional hybrid protein indicating that the fusion did not affect the biochemical activity and the processing of Gas1p.
Localization of Gas1 protein in Saccharomyces cerevisae during vegetative growth:

During vegetative growth mRFPGas1p was present in four distinct locations. As expected it labelled the plasma membrane but surprisingly it was detected also in the chitin ring, in the septum region and in the bud scars where it remained also after several generations. Similar results were obtained in another work performed in parallel in our laboratory using a different fluorescent version protein of Gas1. In this second construct GFP was inserted in the serine-rich region that proceeds the GPI-anchoring signal at the C- terminal end of the protein. This site was chosen since the serine rich region is dispensable for the activity and is a disorder region of the Gas1 protein (Popolo, Ragni et al. 2008). The construct was placed on episomic plasmid, both single and multi-was expressed both in a gas $1 \Delta$ null mutant derived from the diploid strain AN120 and in the parental strain. The same pattern of localization observed with mRFP-Gas1p was detected also with the Gas1-GFP hybrid protein. Moreover, the same localization was maintained when the hybrid GAS1-GFP gene was placed either on single copy or multicopy plasmids. These results 
clearly validate the distinct locations of Gas1 protein that are referred in this thesis. Thus, independently of the type of fluorescent protein, the site of fusion, genetic background or diploidity and gene dosage of the construct, Gas1p maintains the same localization in yeast cells.

\section{Identification of a fraction of mRFP-} Gas1 protein that is covalently bound to the cell wall:

In this work we have observed that mRFP-Gas1 protein is present in two different pools: a mobile one that is located in the plasma membrane and immobile one that is covalently linked to the cell wall where is protected from degradation. The presence of Gas1p in the cell wall was first described in an study in which the protein was recovered from the cell wall by $\beta$-(1,3)-treatment (De Sampaio, Bourdineaud et al. 1999). Moreover, proteomic analyses of the cell wall have also demonstrated that Gas1p, together with Gas3p and Gas5p, is covalently linked to the cell wall (Yin, de Groot et al. 2005). A quantitative analysis has indicated the presence of a low number of Gas1p molecules in the cell wall (Yin, de Groot et al. 2007), supporting the notion that the cell wall is not the primary localization of Gas1p. Its detection in the cell wall has been controversial. The presence of Gas1p in the cell wall proteome could be due to the high level of expression of the protein that might cause some leakiness in the mechanism of retention of proteins in the plasma membrane and also to the high sensitivity of the mass spectrometry technique. However, an alternative hypothesis postulated that Gas1p could be distributed between the plasma membrane and the cell wall, due to the presence of a long Ser-box just upstream of the $\omega$-site that was proposed to affect the distribution of GPI proteins between the cell wall and the membrane (Frieman and Cormack 2004).

With this work we have validated the hypothesis that a form of Gas1p is covalently bound to the cell wall. We have provided evidence of its incorporation at specific sites in the cell wall. We have also exploited the instability of mRFP-Gas1p to analyze the localization of the Gas1p form that is specifically linked to the bud scar. By treating the cells with $\alpha$-Factor, a pheromone that arrest the cells in G1, we could switch off the expression of GAS1. We found that $\alpha$-Factor treatment induced a rapid degradation of mRFPGas1p and disappearance from the cell contour. Interestingly, the protein 
remained in the bud scars. Since at early stages Gas1p was localized in the chitin ring, we conclude that Gas1p could be already cross-linked to the chitin ring from which the bud scar originates. In Western Blot analysis of subcellular fractions of alpha-Factor treated cells mRFP-Gas1p was not detected in either the total or membrane fractions whereas Gas1p was enriched in the membrane fraction. In addition, both Gas $1 p$ and mRFP-Gas1p were detected in the material released after enzymatic treatment with $\beta$-(1,3)-glucanase indicating that the acceptor molecule of Gas 1p cross-linked to the chitin of the bud scar is $\beta$-(1,3)-glucan. Other biochemical analyses have been performed in our laboratory in which the nature of the anchoring have been further analyzed (Rolli, Ragni et al., 2009).

Incorporation of cell wall protein in a localized manner has been demonstrated for three structural mannoproteins, Tip1p, Cw1p and Cw2p. Tip1p was found only in mother cells whereas $\mathrm{Cw} 2 \mathrm{p}$ was incorporated in small-to medium-sized buds. The localization of these proteins was shown to be completely determined by the timing of transcription during the cell cycle. $\mathrm{Cw} 1 \mathrm{p}$ was found to be localized in the birth scar. Localization of Cwp1p depends not only on a cell cycle-regulated expression but also on the timing of septum formation, composition of the septum, or both. (Smits, Schenkman et al. 2006). Synthesis of Gas1p has been found to be maximal at the G1-to-S transition being coincident with the moment of chitin formation at bud emergence (Popolo, Cavadini et al. 1993; Ram, Brekelmans et al. 1995). The incorporation of Gas1p into the chitin ring could be determined by this pattern of expression. However, other mechanisms cannot be ruled out. Mechanisms that depends on actin cytoskeleton and septin ring organization, as occurs with the GPIprotein Crh2p (Rodriguez-Pena, Rodriguez et al. 2002) should be also considered. 


\section{LOCALIZATION OF GAS1 BASED ON THE RESULTS OBTAINED IN OUR LABORATORY:}

The results reported here for the localization of mRFP-Gas $1 p$, together with other results obtained in the laboratory, have been recently published in an article in which we have demonstrated that Gas1p is present in distinct locations (Rolli E. 2009).

Figure 9 shows a scheme of the localization of Gas $1 p$ according to the results that have been obtained. Gas $1 p$ is presented microdomains in the plasma membrane, in the chitin ring and in the septum. After citokinesis, it remains in the bud scars. Moreover, our results have revealed that the immobilization of Gas $1 \mathrm{p}$ at specific sites is required for proper morphogenesis.

Rolli, Ragni et al. 2009 is enclosed at the end of this chapter

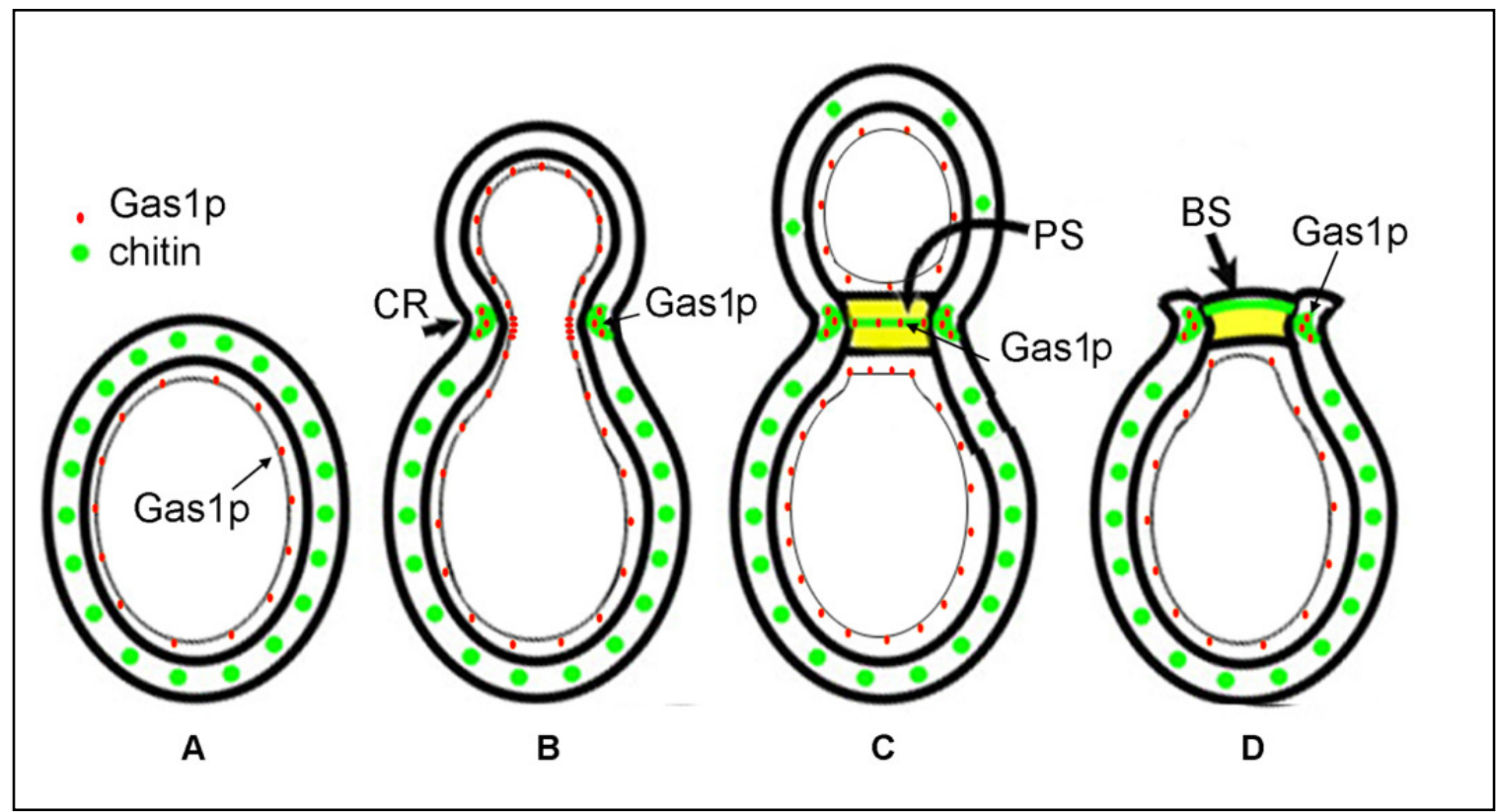

Figure 9. Scheme of the localization of Gas1p. (A) In a new born cell Gas1p (red dots) localizes in microdomains of the plasma membrane. In our laboratory Gas1p has been found in the lipid raft fraction by making biochemical studies using a Gas1p-GFP construction confirming previous cell wall proteomic analyses. (B) In a small-budded cell, Gas1p is in the plasma membrane but is also linked to the chitin ring presumably through a transglycosidase reaction which is likely to take place at the neck region sometime during the cell cycle. (C) In large-budded cells Gas1p is localized at, or in close proximity to, the primary septum. This linkage is unknown. (D) Gaslp remains in the bud scar and all over the plasma membrane. CR: chitin ring, PS: primary septum, BS: bud scar. 
Immobilization of the Glycosylphosphatidylinositol-anchored Gas1 Protein into the Chitin Ring and Septum is required for proper morphogenesis in Yeast.

Rolli E, Ragni E, Calderon J, Porello S, Fascio U, Popolo L.

Mol Biol Cell. November 19, 2009 


\section{GENERAL CONCLUSIONS:}

\section{In Candida albicans:}

1. Phr1-GFP protein is present in discrete points on the cell surface and on lipid rafts during vegetative growth

2. Phr1p localizes at the cell periphery, in the neck region, in the septum and in bud scars during vegetative growth

3. Phrlp co-localizes with the chitin at the level of the chitin ring, in the bud scars and in the septum

4. Phrlp is required for strengthening the neck region during cell division

5. Phr1-GFP protein concentrates at the tip of the germ tubes at the beginning of the filamentous growth and when the germ tubes elongates the fluorescent protein remains at the apex of the hypahe but it also gradually distribute along the lateral walls of the hyphae as they elongate

6. Phr1 protein is highly polarized during hyphal growth and it is required at the site of maximal growth probably due to the incorporation and elongation of newly synthesized $\beta$-(1,3)-glucan in the expanding cell wall

7. The localization of Phrlp in the tips of the germ tubes and in the lateral walls of the hyphae does not depend on microtubules but a correct polymerization of microtubules is required for the proper localization in the septa of the hyphae

8. In yeast cells actin cystoskeleton is essential for correct transport of Phr1-GFP protein to the cell surface and during hyphal growth actin is required for a correct polarized localization of Phr1-GFP

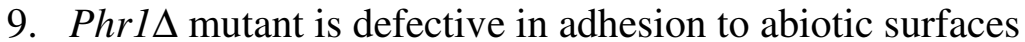

10. Loss of Phr1p strongly reduces adhesion of Candida albicans cells to monolayers of human epithelial cells

11. Transcript levels of $A L S 3$ and EAP1 genes are not affected by the deletion of PHRl gene during the conditions of adhesion to an abiotic surface. By contrast, the deletion of PHRI gene causes a marked reduction of $H W P 1$ and ECE1 transcripts but the downregulation of these genes is not specific to the adhesion condition

12. Phrls mutant is unable to invade reconstituted human epithelia 
In Saccharomyces cerevisiae:

1. Gas1 protein is localized at the cell periphery, in the chitin ring and in the septum and after cytokinesis it remains in the bud scars

2. The presence of Gas1p in the chiting ring could be necessary to maintain the rigidity required in the neck region

3. mRFP-Gas1 fusion protein is present in two different pools: a mobile one that is located in the plasma membrane and an immobile one that is protected from degradation

4. The immobile fraction of Gas $1 p$ is covalently linked to the cell wall 


\section{Candida albicans COMO PATÓGENO FÚNGICO:}

C. albicans es una especie del género Candida que se encuadra dentro del Phylum Ascomycota. C. albicans es un organismo diploide que aparentemente carece de ciclo sexual. Sin embargo, en los últimos años varios estudios han demostrado evidencias que sugieren la posible existencia de un ciclo sexual en esta levadura (Tzung, Williams et al. 2001; Bennett and Johnson 2005).

C. albicans tiene un genoma diploide que se haya dividido en 8 pares de cromosomas. El genoma de C. albicans es ligeramente mayor que el de $S$. cerevisiae, probablemente por la presencia de una enorme cantidad de familias de retrotransposones. Además contiene varias familias de genes que codifican proteasas, lipasas y proteínas de pared celular que no están presentes con tal redundancia en el genoma de $S$. cerevisiae (Berman and Sudbery 2002).

\subsection{PATOGENICIDAD}

\section{FACTORES DE VIRULENCIA}

En los últimos años se ha producido un notable incremento de las infecciones fúngicas, siendo C. albicans el principal agente etiológico de este tipo de infecciones. Se trata de un patógeno oportunista que coloniza las superficies mucosas tanto de cavidades orales y vaginales como del tracto gastrointestinal siendo también capaz de causar una gran variedad de infecciones, según las condiciones de hospedador (Mavor, Thewes et al. 2005).

Las infecciones por Candida (candidiasis) se clasifican en superficiales y profundas. La infección se produce, normalmente, por una alteración del equilibrio entre el hospedador y la levadura. Las infecciones sistémicas por Candida son particularmente difíciles de diagnosticar y tratar, resultando en ocasiones fatales. (Mavor, Thewes et al. 2005; KarkowskaKuleta, Rapala-Kozik et al. 2009).

El tratamiento de las infecciones fúngicas, y especialmente el de las sistémicas, es difícil debido al gran parecido entre el hospedador y el hongo, ambos eucariotas. Las terapias actuales para el tratamiento de las micosis invasivas sólo abarcan un reducido número de antifúngicos como la anfortericina B o el fluconazol. Además, en la última década se ha experimentado un incremento significativo de la resistencia a agentes antifúngicos que conlleva implicaciones importantes en la morbilidad, mortalidad y el coste sanitario. En los últimos años se han 
desarrollado agentes de nueva generación así como de familias químicas ya empleadas como el voriconazol, la caspofungina o la micafungina. Sin embargo, aún es necesario revisar nuevas dianas antifúngicas y proponer terapias combinadas (Gauwerky, Borelli et al. 2009).

El potencial patogénico del C.albicans está determinado por la combinación de diversos factores de virulencia entre los que destacan: el fenómeno de la transición fenotípica ("phenotypic switching"); el dimorfismo o transición dimórfica entre las formas de levadura e hifa; la capacidad de adhesión o la producción de enzimas hidrolíticas tales como proteinasas, lipasas o fosfolipasas (Calderone and Fonzi 2001; Brown, Odds et al. 2007).

\subsection{CICLO BIOLÓGICO Y POLIMORFISMO}

C. albicans puede considerarse un hongo polimórfico ya que es capaz de crecer en una gran variedad de formas morfológicas que abarcan desde formas unicelulares, como levaduras o clamidosporas, a formas filamentosas como hifas o pseudohifas (Sudbery, Gow et al. 2004). Un componente importante de dicha versatilidad es la capacidad de sobrevivir como comensal en diversos lugares del hospedador en los cuales $C$. albicans debe ser capaz de adaptarse a una gran variedad de presiones fisiológicas.

En particular, la transición entre las formas de levadura y de hifa, también denominada dimorfismo, se considera un importante factor de virulencia. Tanto hifas como pseudohifas son invasivas siendo capaces de invadir, por ejemplo, una matriz de agar cuando crecen en placas de laboratorio. Dicha propiedad puede promover la penetración en los tejidos del hospedador durante las etapas iniciales de la infección siendo importante para la colonización de los órganos del hospedador. La forma de levadura, por su parte, puede ser más apropiada para la diseminación en el torrente sanguíneo. La transición dimórfica, por tanto, juega un papel esencial en la virulencia de $C$. albicans (Sudbery, Gow et al. 2004; Whiteway and Oberholzer 2004).

La transición dimórfica es un proceso reversible que puede inducirse in vitro en respuesta a diversos factores tales como, la presencia de suero, Nacetylglucosamina (GlcNAc), $\mathrm{pH}$ (ardedor de 7- 7.5), altas temperaturas $\left(37^{\circ} \mathrm{C}\right)$ o presencia de $\mathrm{CO}_{2}$. Dichas condiciones de inducción reflejan la enorme variedad de señales que 
promueven la filamentación en distintos ambientes in vivo.

Regulación transcripcional de la transición dimórfica:

Entre los hongos existe un alto grado de conservación de los mecanismos que conectan las señales ambientales con los cambios en diferenciación celular. Muchos de los genes necesarios para los procesos de polarización en Saccharomyces cerevisiae están conservados en Candida albicans. La conservación de esta maquinaria sugiere que el desarrollo de hifas en C. albicans no es un totalmente nuevo sino que representa una modulación de los mecanismos que controlan el crecimiento polarizado (Sanchez-Martinez and Perez-Martin 2001; Whiteway and Oberholzer 2004).

En el proceso de inducción de la filamentación, diversos factores activan, a través de proteínas sensoras, diferentes rutas de transducción de señales. Dichas de transducción señalización confluyen en factores de transcripción que regulan la expresión de genes diana específicos de las hifas (Ernst 2000). Según distintos modelos, las vías de señalización pueden converger en factores de transcripción distintos o idénticos que regulan la expresión de genes específicos de las hifas. Las distintas vías de señalización forman una compleja red que integra las señales ambientales del exterior para un adecuado control de la transcripción y la morfogénsesis.

La Figura 5 (Introduction) muestra un esquema de las distintas vías de transducción de señales que regulan la transición dimórfica en C. albicans.

Los genes específicos de hifas (HSGs, del inglés: hypha-specific genes) identificados hasta el momento incluyen: HWP1, ECE1, HYR1, ALS3, ALS8, RBT1 y RBT4. Dichos genes codifican proteínas de pared celular o proteínas de secreción que se ha demostrado son importantes factores de virulencia en las infecciones sistémicas (Calderone and Fonzi 2001; Yang 2003; Brown, Odds, et al 2007).

\section{Checkpoint morfogenético y ciclo celular:}

Dada la importancia de los procesos morfogenéticos para la virulencia de $C$. albicans es importante conocer la relación entre ciclo celular y morfología y la manera en que dichas relaciones regulan la transición morfológica.

El crecimiento polarizado se consigue en $C$. albicans, al igual que en otros hongos, mediante la localización del citoesqueleto de actina y el transporte de 
los materiales necesarios para el crecimiento celular hacia puntos concretos de la superficie. Por ello, durante el ciclo celular tienen lugar cambios en el citoesqueleto de actina y en los microtúbulos que están correlacionados con los eventos más importantes del crecimiento polarizado de la célula. (Finley and Berman 2005).

\section{El citoesqueleto de actina:}

Durante el desarrollo hifal, la expansión de la superficie celular se haya restringida al ápice de las hifas. El citoesqueleto de actina es importante para dicho crecimiento polarizado.

Las células levaduriformes de $C$. albicans presentan un cambio temporal en la organización del citosqueleto de actina durante el ciclo celular que se asemeja al de $S$. cerevisiae mientras que el cistosqueleto de actina se encuentra altamente polarizado durante el crecimiento hifal (Sudbery, Gow et al. 2004). La organización de los filamentos y "patches" de actina en el ciclo celular de las levaduras y en el primer ciclo celular de pseudohifas e hifas se encuentra esquematizada en la figura 6 (Introduction).

\section{Los microtúbulos:}

Una diferencia clara entre las distintas formas celulares de C. albicans es la coordinación espacial de la dinámica nuclear y la morfología. La dinámica nuclear es más dramática en las hifas donde el núcleo migra en los tubos germinativos y se divide a través del futuro sitio de reptación unas 10-20 $\mu \mathrm{m}$ más allá de la célula madre. La manera en que los núcleos migran hacia el sitio de septación no es del todo clara. Sin embargo, se sabe que para dicha migración es necesario el citoesquelo de microtúbulos (MTs). Los microtúbulos en $C$. albicans están implicados en el posicionamiento del núcleo y en la regulación de la morfogénesis (Finley and Berman 2005).

La Figura 7 (Introduction) muestra un modelo de la dinámica nuclear y de los microtúbulos durante el ciclo celular en el proceso de crecimiento hifal.

\section{4 .} HOST-PATHOGEN

\section{INTERACTION}

Las infecciones por Candida se producen cuando existe un desequilibrio entre las defensas del hospedador y el microorganismo. Comprender las relaciones entre el patógeno y el hospedador y los mecanismos que influencian el paso de comensal a 
patógeno es crucial para establecer las bases del potencial de $C$. albicans como patógeno.

Uno de los primeros pasos en el proceso de infección es la unión a las superficies tisulares del hospedador, proceso al que le sigue la invasión de los tejidos internos (Rupp, S. 2007). Así, adhesión e invasión son dos procesos esenciales para el establecimiento de la infección.

\section{Adhesión a las superficies del hospedador:}

Candida albicans expresa varias adhesinas entre las que se incluyen proteínas Als, Hwp1p, Eap1p, Csh1p así como otras proteínas de superficie celular que promueven la unión a los tejidos del hospedador.

La familia de genes $A L S$ codifica proteínas que median la adhesión a los epitelios, la agregación de levaduras y la formación de biofilms. La familia está compuesta por ocho genes, ALS1-7 y $A L S 9$. Diversos estudios han demostrado la expresión de todos los genes $A L S$ en aislados clínicos y en modelos murinos de infección. Sin embargo, la expresión de los genes $A L S 1, A L S 3$ y $A L S 9$ se ha detectado con mayor frecuencia. (Biswas, Van Dijck et al. 2007; Karkowska-Kuleta, Rapala-Kozik et al.
2009). La proteína Hwp1 de C. albicans, es una proteína anclada mediante GPI a la pared celular que se sabe es un susbstrato para las transglutaminasas de mamífero. Hwp1p participa en las uniones covalentes entre el hongo y el epitelio del hospedador. Otras adhesinas conocidas en $C$. albicans son Eap1p y Csh1p. Eap1p media la adhesión a las superficies plásticas y por tanto dicha proteína se considera puede ser crucial en las candidiasis inducidas a partir de prótesis y catéteres. Por su parte la proteína Chs1p de C. albicans se sabe que aumenta la hidrofobicidad de las células de $C$. albicans facilitando de esta manera las interacciones receptorlingando entre el patógeno y el hospedador (Karkowska-Kuleta, RapalaKozik et al. 2009).

\section{Proceso de invasión:}

En $C$. albicans se conocen diversos factores que se sabe son necesarios para que el hongo se convierta en patógeno. El proceso de invasión intuitivamente parece ser facilitado por la transición entre las formas de levadura y de hifa. Sin embargo, dicha afirmación es sólo "parcialmente" cierta dado que existen otros mecanismos tales como la persopción de células levaduriformes en la mucosa intestinal hacia los tejidos 
internos así como la fagocitosis inducida por células esofágeas (Calderone and Fonzi 2001). Además, deben de considerarse otros factores, tales como el proceso de adhesión y las adhesinas que son necesarias en un primer momento en las interacciones patógeno-hospedador así como la actividad de enzimas hidrolíticas que contribuyen a la invasión tisular mediante la digestión de membranas celulares y por la degradación de moléculas de la superficie del hospedador (Staib, Kretschmar et al. 2000; Calderone and Fonzi 2001; Schaller, Borelli et al. 2005).

\section{THE FUNGAL CELL WALL}

Los hongos y levaduras están rodeados de una pared celular, una estructura compleja esencial para el mantenimiento de la forma celular, la prevención de la lisis y la protección frente a condiciones externas adversas. Según esta definición la pared celular parece ser una estructura estática. Sin embargo, la pared celular está continuamente cambiando de forma y de tamaño para acompañar el crecimiento celular y además debe ser remodelada durante los procesos morfogenéticas (Cabib, Drgon et al. 1997; Lagorce, Hauser et al. 2003).
Es importante destacar que la pared celular fúngica es una estructura esencial para los hongos pero que está ausente en las células eucariotas superiores, lo que la convierte en una atractiva diana para nuevos antifúngicos.

\subsection{ESTRUCTURA Y} COMPOSICIÓN DE LA PARED CELULAR:

La pared celular ha sido estudiada en gran detalle en $S$. cerevisiae y su bioquímica, fisiología y estructura han sido ampliamente revisadas aun cuando la comprensión de aspectos como la interacción de los distintos componentes y su ensamblaje es aún limitada.

S. cerevisiae consume una gran cantidad de energía metabólica en la construcción de su pared celular que representa aproximadamente el $20 \%$ de su peso seco. La pared celular está constituída principalmente por tres macromoléculas: $\beta$-glucano -formado por enlaces $\beta-(1,3), \beta-(1,6)$, o ambos-, quitina y manoproteínas. La estructura de la pared se encuentra ilustrada en la Figura 9 (Introduction). Básicamente, las cadenas de $\beta$-(1,3)-glucano se encuentran ramificadas con enlaces $\beta-(1,6)$ formando un matriz fibrilar a la que se unen quitina, $\beta-(1,6)$ y manoproteínas. 
Esta estructura está dispuesta en capas, según se observa mediante microscopía electrónica de transmisión. Las fibras insolubles de $\beta$-(1,3)-glucano se distribuyen alrededor de la superficie de la célula, uniéndose mediante un enlace covalente a las cadenas de quitina $\mathrm{y}$ conformando así la parte interna de la pared celular. Hacia el exterior de la pared se sitúan las manoproteínas unidas al extremo no reductor de las moléculas de $\beta$-(1,3)-glucano, bien directamente, como es el caso de las proteínas Pir, bien indirectamente a través de una molécula de $\beta$-(1,6)-glucano en el caso de las proteínas GPI. También están presentes en la pared proteínas unidas a quitina mediante cadenas cortas de $\beta$-(1,6)glucano, aunque en menor proporción (Kollar, Reinhold et al. 1997; de Groot, de Boer et al. 2004; Lesage and Bussey 2006).

La organización de la pared celular de Candida albicans es similar a la de Saccharomyces cerevisiae presentando también una doble capa cuando se observa al microscopio electrónico de transmisión. Sin embargo, existen algunas diferencias en cuanto a la composición y biosíntesis de la pared celular. Así, por ejemplo, C. albicans presenta una cantidad de $\beta$-(1,6)-glucano en su pared celular que es aproximadamente el doble de la que presenta S. cerevisiae (Mio, YamadaOkabe et al. 1997). Además, en $C$. albicans distintas evidencias apoyan la idea de que el $\beta$-(1,6)-glucano es una prometedora diana para el desarrollo de nuevos antifúngicos (Kitamura, Someya et al. 2009).

\section{LA FAMILIA DE PROTEÍNAS GH72}

La familia de proteínas Gel/Gas/Phr de Aspergillus fumigatus, Saccharomyces cerevisiae y Candida albicans desempeña un papel fundamental en la biogénesis de la pared celular actuando como enzimas que procesan el $\beta$-(1,3)glucano (Mouyna, Fontaine et al. 2000). Las enzimas pertenecientes a dicha familia catalizan una reacción in vitro que consiste en la ruptura de una unión glicosídica interna de las cadenas de $\beta$ (1,3)-glucano, la liberación del extremo reductor y la transferencia del nuevo extremo reductor al extremo no-reductor de otra molécula de $\beta$-(1,3)-glucano que actúa como aceptor. Hasta el momento se han incluido 94 proteínas de distintos orígenes que en conjunto forman la familia GH72 de las Glicosil Hidrolasas (GHs)

(http://afmb.cnrs- 


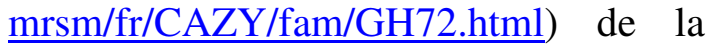

base de datos Pfam

(http://www.sanger.ac.uk//cgi-

bin/Pfam/getacc??PF03198). Dichas

proteínas comparten algunas

características estructurales comunes.

Todas ellas son proteínas modulares que poseen un dominio catalítico común de aproximadamente 310 aa (dominio GluTD, de Glucanosyl-Transferase Domain) así como un péptido señal en el extremo amino. La parte central de estas proteínas contiene un segmento de unión ("linker") y el extremo carboxílico incluye una región rica en serinas y una secuencia señal para la unión del motivo GPI (Ragni, Fontaine et al, 2007).

Diversos estudios han demostrado que en $A$. fumigatus y en $C$. albicans, la disrupción de homólogos de los genes que codifican para dichas proteínas (genes GEL y $P H R$ respectivamente) conduce a una notable reducción en la virulencia en modelos animales de infección (De Bernardis, Muhlschlegel et al. 1998; Mouyna, Morelle et al. 2005). Dada su importancia en el ensamblaje de la pared celular y en la virulencia las proteínas de dicha familia son prometedoras dianas para nuevos antifúngicos.

\subsection{FAMILIA MULTIGÉNICA GAS} DE S. cerevisiae.

La familia de genes $G A S$ de $S$. cerevisiae está constituida por cinco miembros, GAS1-5. La familia de genes $G A S$ desempeñana un papel fundamental en el ensamblaje y la remodelación de los componentes de la pared celular actuando $\quad$ como $\quad \beta-(1,3)$ glucanosiltranferasas. GAS1, es el miembro mejor caracterizado de la familia y se expresa, junto a GAS5, exclusivamente durante el crecimiento vegetativo, encontrándose reprimido durante la meiosis y la esporulación. GAS2 y GAS4 presentan un patrón de expresión inverso encontrándose inducidos durante la meiosis y la esporulación y reprimidos durante el crecimiento vegetativo. GAS3 se encuentra débilmente expresado durante el crecimiento vegetativo y débilmente inducido durante la esporulación (Ragni, Coluccio et al. 2007).

La secuencia de los cinco ortólogos de las proteínas Gas ha sido ampliamente analizada en nuestro laboratorio. La Figura 11A (Introduction) muestra una representación esquemática de la arquitectura modular de las proteínas Gas. Las proteínas Gas comparten un péptido señal en el extremo amino y un domino amino terminal, denominado $\mathrm{NtD}$ de aproximadamente 330-350 
aminoácidos. El dominio NtD es el putativo domino catalítico ya que contiene dos residuos de glutamato, conservados en todas las proteínas Gas así como en todos los miembros de la famila GH72, que son esenciales para la catálisis (Kapteyn, Ram et al. 1997; Mouyna, Fontaine et al. 2000; Carotti, Ragni et al. 2004). Dicho dominio está anotado en la base de datos Pfam como Glyco_hydro_72 (GH72). Un segmento de unión ("linker") rico en aminoácidos flexibles, tales como T,G,A,P y S y que contiene un residuo de cisteínas altamente conservdo une el dominio $\mathrm{NtD}$ con la región C-terminal. La región Cterminal tiene una extensión variable y difiere considerablemente entre las distintas proteínas Gas excepto entre Gas1p y Gas2p. Gas1p y Gas $2 p$ comparten una elevada identidad aminoacídica en la región C-terminal (41\%) debido a la presencia de un dominio denominado Cys-box de aproximadamente 100 aminoácidos. El domino Cys-box se encuentra anotado en la base de datos Pfam como PfamX8. Dicho dominio ha sido recientemente clasificado como CBM43 dentro del CBMs (del inglés "carbohydrate binding domains") de la base de datos CAZy (del inglés "carbohydrate active enzymes"). En una publicación reciente en la que ha participado nuestro laboratorio la Familia
GH72 ha sido clasificada en dos subfamilas, $\mathrm{GH}_{72}{ }^{+}$y $\mathrm{GH} 72^{-}$, que se disferencian en la presencia o ausencia del dominio rico en cisteínas, Cys-box (Ragni, Fontaine et al. 2007). Además, todas las proteínas Gas contienen una secuencia de anclaje a GPI. Dicho motivo es una señal para la localización de proteínas en la membrana plasmática (Caro, Tettelin et al. 1997; Frieman and Cormack 2003).

\section{Fenotipo del mutante gas1 $1 \Delta$ :}

La ausencia de la actividad glucanosiltransferásica de Gas1p causa una morfología celular aberrante y un daño celular transitorio. Las células que carecen de la proteína Gas1 presentan una reducción en la tasa de crecimiento del 15 al $40 \%$ y se presentan como células redondeadas, engrosadas, altamente vacuolazas y con múltiples yemas (Popolo, Vai et al. 1993).

La pared celular de las células mutantes de GASl está también afectada: la permeabilidad a las substancias externas aumenta, las células son más sensibles a agentes externos que perturban la pared celular tales como el calcofluor white, y la resistencia a enzimas hidrolíticas es elevada y similar a la de las células en fase estacionaria (Ram, Wolters et al. 1994; Popolo, 
Gilardelli et al. 1997). Dichos defectos reflejan modificaciones en la organización y composición de la pared celular. De hecho, la ausencia de Gas1p conduce a una disminución en la incorporación de nuevas cadenas de glucano en la pared preexistente y a una disminución en el ratio glucano alkalisoluble/-insoluble. Así mismo, se produce un aumento en el contenido de quitina y los defectos en el ensamblaje de la pared dan lugar a la liberación de manoproteínas en el terreno de cultivo. (Popolo, Gilardelli et al. 1997; Valdivieso, Ferrario et al. 2000).

La debilitación de la pared celular induce un mecanismo compensatorio ("integrity pathway"), una ruta de MAP kinasas, que conlleva al reenforzamiento de la pared celular (Garcia, Bermejo et al. 2004).

\subsection{FAMILIA DE PROTEÍNAS Phr DE Candida albicans:}

Los primeros homólogos de genes GAS descritos en Candida albicans fueron los genes PHR1 y PHR2 (Saporito-Irwin, Birse et al. 1995; Muhlschlegel and Fonzi 1997). Las proteínas Phr1p y $\mathrm{Phr} 2 \mathrm{p}$ son proteínas ancladas a la superficie celular mediante un motivo GPI que procesan el $\beta$ - $(1,3)$ glucano de una reacción similar a la que catalizan las proteínas Gas en $S$. cerevisiae. Más tarde, tres nuevos homólogos de los genes GAS fueron identificados: PHR3, PGA4 y PGA5 (Eckert, Heinz et al. 2007).

El patron de expression de los genes PHR1 y PHR2 depende del $\mathrm{pH}$ ambiental. Así, in vitro, la expression de PHRI se detecta cuando el $\mathrm{pH}$ ambiental es mayor de 5.5 incrementando su expression con $\mathrm{pH}$ más alcalinos. $P H R 2$ presenta un patron de expresón opuesto encontrándose expresado a valores de $\mathrm{pH}$ por debajo de 6 (Saporito-Irwin, Birse et al. 1995; Muhlschlegel and Fonzi 1997; Fonzi 1999). Por su parte, la expresión de los genes PHR3, PGA4 Y PGA5 es independiente del $\mathrm{pH}$. La expresión de PHR3 y PGA5 es constitutivamente baja mientras que PGA4 muestra liveles de expresión constitutiva fuertes (Eckert, Heinz et al. 2007).

Cabe destacar que los genes PHRI y PHR2 son fundamentals para una correcta morfogénesis y que la ausencia de dichos genes conduce a una reducida virulencia en modelos animals de infección (Ghannoum, Spellberg et al. 1995; De Bernardis, Muhlschlegel et al. 1998). 
Fenotipo del mutante phr1s y su relación con la virulencia:

La ausencia de PHRI provoca defectos morfológicos tanto en el crecimiento en forma de levadura como en el crecimiento filamentoso. Cuando el mutante phrls crece a $25^{\circ} \mathrm{C}$ a $\mathrm{pH}$ alcalino las levaduras presentan una morfología aberrante con formas redondeadas y alargadas después de largos periodos de incubación. Periodos de incubación más cortos resultan en defectos morfológicos más sutiles. Durante el crecimiento en medio alcalino, las yemas que se forman en el mutante son defectuosas presentando una forma más redondeada y aplanada en comparación con las yemas de la cepa parental (Saporito-Irwin, Birse et al. 1995). Los defectos en filamentación del mutante phrl $\Delta$ son también dependientes del pH. A pH 6 el mutante no presenta diferencias en cuanto a la frecuencia o morfología de los tubos germinativos que dan lugar a las hifas. Sin embargo, a valores de $\mathrm{pH}$ mayores, progresivos aumentos en el $\mathrm{pH}$ dan lugar a una correspondiente reducción en el tamaño de los tubos germinativos y en la expansión del ápice de los mismos. A pH 8, dicho patrón culmina con la aparición de tubos germinativos distorsionados que no llegan a alargarse y aparecen muchas célululas que adoptan morfologías similares a smhoos o formas aberrantes que se han denominado "peanut-shaped" (similares a cacahuetes). Por tanto, el $\mathrm{pH}$ del medio altera la capacidad del mutante phrls para mantener y llevar a cabo un crecimiento polarizado tanto el crecimiento levaduriforme como durante el crecimiento hifal (Saporito-Irwin, Birse et al. 1995).

El mutante nulo de PHRI es avirulento en modelos de infección sistémica mientras que la capacidad de causar infección vaginal en ratas no se encuentra alterada (De Bernardis, Muhlschlegel et al. 1998). Dado que el pH sanguíneo es cercano a la neutralidad el $\mathrm{pH}$ vaginal es aproximadamente 4.5, los defectos en virulencia reflejan la dependencia del $\mathrm{pH}$ de los defectos morfológicos observados in vitro que se corresponden con el patrón de expresión del gen.

\section{La respuesta al $\mathrm{pH}$ en Candida albicans:}

La capacidad de $C$. albicans de responder a cambios en el $\mathrm{pH}$ extracelular es crucial para su supervivencia en diversos ambientes y, consecuentemente, para su potencial como patógeno (Davis 2003). 
En Ascomicetes, entre los que se incluye $C$. albicans, y en basidiomicetes se ha descrito una vía de señalización que regula la adaptación a $\mathrm{pH}$ neutro-alcalino (Davis, Wilson et al. 2000; Davis 2003; Kullas, Martin et al. 2007). Dicha ruta de señalización está controlada por el factor de transcripción Rim101/PacC. Rim101 se une a los promotores induciendo la expresión de genes que se expresan preferencialmente a $\mathrm{pH}$ ácido (Baek, Martin et al. 2006; Aronova, Wedaman et al. 2007). La actividad de Rim101 está controlada por la eliminación proteolítica de una porción de la región C-terminal (Li, Martin et al. 2004). En ambientes ácidos Rim101 es procesado a una forma inactiva en $C$. albicans mientras que en S. cerevisiae no se procesa. En ambientes neutros-alcalinos Rim101 es procesado a una forma activa tanto en $S$. cerevisiae como en $C$. albicans. Para el procesamiento de Rim101 son necesarios otros miembros de la vía de señalización, entre los que se encuentran Rim8/PalF, Rim13/PalB, Riom20/PalA, Rim21/PalH, Dfg16 y Snf7 (Futai, Maeda et al. 1999; Porta, Ramon et al. 1999; Davis, Wilson et al. 2000; Davis, Bruno et al. 2002; Davis 2003; Kullas, Martin et al. 2007). 


\section{OBJETIVOS DE ESTA TESIS:}

El objetivo de esta tesis fue la caracterización funcional de dos proteínas contenientes glisosilfosfatidilinositol (GPI), Phr1p en el patógeno fúngico Candida albicans y su homólogo Gas1p en la levadura de gemación Saccharomyces cerevisiae. Mediante el uso de técnicas de fusión de proteínas fluorescentes pretendíamos llevar a cabo un estudio de la localización de CaPhr1p y ScGas1p con el propósito de analizar la función de dichas proteínas de membrana en distintos procesos morfogenéticas. Además, nuestro objetivo fue también el estudio del papel de Phrlp, un importante factor de virulencia, en la patogénesis de $C$. albicans mediante el análisis de su contribución a la adhesión e invasión, dos procesos fundamentales en el establecimiento de la infecciones por Candida albicans.

En concreto, los objetivos del trabajo fueron:

1. El análisis de la localización dinámica de la proteína Phr1p durante el crecimiento en forma levaduriforme y durante la filamentación en C. albicans

2. El uso de modelos de tejido reconstituído para el análisis in vitro de la capacidad del mutante phrls de C. albicans para adherir e invadir tejidos humanos

3. El análisis de la localización de la proteína Gas1p durante el crecimiento vegetativo en $S$. cerevisiae

4. El estudio de la localización de membrana y del anclaje a la pared celular de la proteína Gas1p en $S$. cerevisiae para determinar si la pared celular es un destino específico de la proteína 
LOCALIZACIÓN DE LA

PROTEÍNA Phr1p DE Candida

albicans DURANTE EL

CRECIMIENTO EN FORMA

DE LEVADURA Y DURANTE

LA FILAMENTACIÓN

\section{ANTECEDENTES:}

La pared celular de $C$. albicans está en contacto íntimo con las células del hospedador y es altamente dinámica, respondiendo a cambios externos, crecimiento celular y condiciones de esters. Además, la pared celular de $C$. albicans debe remodelarse con los cambios morfogenéticos del hongo. Alteraciones en la pared celular pueden influir en la capacidad de Candida albicans para evadir las defensas del hospedador y para colonizar diferentes nichos (Nather and Munro 2008).

La proteína Phrlp de Candida albicans perteneciente a la Familia GH72 de las Glicosil Hidrolasas, desempeña un papel fundamental en la remodelación de la pared celular siendo necesaria para una correcta morfogénesis (Saporito-Irwin, Birse et al. 1995). Así mismo, Phrlp se considerea un importante factor de virulencia en Candida albicans (Ghannoum, Spellberg et al. 1995; De Bernardis, Muhlschlegel et al. 1998).
La proteína Phrlp tiene una sequencia señal de anclaje a GPI en su extremo carboxi-terminal. El anclaje de proteínas por medio de GPI en células eucariotas se considera un mecanismo de anclaje de proteínas a la superficie celular. Sin embargo, existen distintas evidencias que indican que la proteína Phrlp no es solo una proteína de membrana sino que se encuentra también unida covalentemente a la pared celular. Así, en estudios de espectrometría de masas Phr1p ha sido identificada como unida covalentemente a la pared celular (de Groot, de Boer et al. 2004).

Es importante considerar que existen diversas razones por las que la proteína Phrlp de Candida albicans se puede considerer una prometedora diana de nuevos antifúngicos.

\section{OBJETIVOS:}

Este trabajo se enmarca dentro de un proyecto más amplio que tiene como objetivo el entendimiento de la function de la proteína Phr1p y su relación con la virulencia de $C$. albicans.

En particular el objetivo de nuestro trabajo fue la caracterización de la localización de la proteína durante el crecimiento en forma de levadura así como durante el crecimiento filamentoso. 
RESULTADOS:

Construcción de una version

functional de la proteína de fusión Phr1-GFP:

Haciendo uso de las técnicas de fusión con proteínas fluorescentes para obtuvimos una fusión de la proteína Phr1 con la Proteína Verde Fluorescente (GFP). Mediante un tagging interno introdujimos la GFP tanto en el extremo amino como en el extreme carboxilo de la proteína. Para la selección del sitio de inserción se utilizó el programa bioinformático GlobPlot (http://globplot.embl.de/) que predice los dominios globulares de las proteínas. De esta manera seleccionamos una region desordenada de la proteína para la inserción de la Proteína Verde Fluorescente (GFP). La inserción en el extremo amino de la proteína se realizó detrás de la secuencia señal entre los aminoácidos S24-S25, mientras que la inserción en la region carboxílica se realizó antes de la señal para la union del GPI entre los aminoácidos G489-G490. Utilizamos una estrategia basada en PCR en la que a partir del plásmido pGUG amplificamos el cassette GFP-URA3GFP. Mediante el análisis de los transformantes obtenidos a partir de los productos de PCR tras su paso por FOA pudimos observar que la fusion al extremo N-terminal daba lugar a una proteína con una fluorescencia débil mientras que la fusion al C-terminal parecía ser óptima para los estudios de localización. La figura 1 (Chapter I) muestra el GlobPlot y el esquema de la fusión al extremo C-terminal.

Mediante un test se sensibilidad a Calcofluor White pudimos determinar que la proteína de fusión era funcional dado que complementaba la hipersensibilidad a Calcofluor White del mutante phrls. Además, mediante Western Blot pudimos confirmar que la proteína de fusión se detectaba tanto durante el crecimiento en forma de levadura como durante el crecimiento filamentoso a $\mathrm{pH}$ alcalino.

\section{La proteína Phr1-GFP localiza en microdominios de membrana:}

En un primer análisis de la localización de la proteina durante crecimiento vegetativo en YPD tamponado a $\mathrm{pH} 7.5$ a $25^{\circ} \mathrm{C}$ observamos que, en celulas en fase exponencial de crecimiento, la proteína Phr1-GFP se encuentra presente alrededor de la superficie celular dando una señal más intensa en la parte del cuello entre la célula madre y la célula hija. De manera interesante, observamos que la fluorescencia en torno a la célula no era uniforme sino que aparecía como una 
serie de puntos intensos (Figura 4A; Chapter I). Dicha observación se confirmaba observando un plano focal diverso en el que la fluorescencia a puntos aparecía en puntos discretos alrededor de las células (Figura 4B; Chapter I). Dicho patrón "puntuado" concuerda con la localización de la proteína en los "lipids rafts" de la membrana. Los lipid rafts son microdominios ricos en esfingolípidos y ergosterol insolubles en detergents noiónicos a bajas temperaturas y por esta característica se denominan "membranas resistentes a detergentes" (DRMs).

Para confirmar dicha localización analizamos lisados celulares después de la extracción con Triton-X100 y tras la centrifugación en gradiente discontínuo. El análisis por Western Blot de las fracciones del gradiente confirmó la detección de la proteína Phr1-GFP así como de la proteína endógena en la fracción de membrana resistente a detergentes (DRMs) (Figura 4C y D; Chapter I).

\section{Localización de la proteina Phr1 durante el crecimiento vegetativo:}

El análisis detallado de la localización de la proteína Phr1-GFP durante el crecimiento vegetativo se obtuvo mediante microscopía confocal. En dichos experimentos se crecieron las células en YPD tamponado a $\mathrm{pH} 7.5$ a $25^{\circ} \mathrm{C}$. Realizamos además una tinción con Calcofluor White para determinar si la proteína colocalizaba con la quitina. Con dichos experimentos observamos que durante el crecimiento vegetativo la proteína Phr1-GFP se encuentra localizada en la superficie celular, de acuerdo con la localización de membrana, y se acumula en puntos específicos (Figura 5A). En células con una yema pequeña, la señal en la yema es más intensa que la señal en la célula madre. Además, la fluorescencia verde aparece en dos puntos brillantes a ambos lados del cuello entre la célula madre e hija y colocaliza con la quitina en el anillo de quitina. Phr1-GFP colocaliza con la quitina también en las cicatrices de gemación (Figura 5B, panel superior). En células con la yema más grande, Phr1GFP da una señal intensa en el septo colocalizando también con la quitina en dicha región (Figura 5B, panel inferior)

\section{La pérdida de PHRI afecta la} longitude del diámtro del cuello:

Con el objetivo de determinar si la proteína Phr1 desempeña un papel biológico en la zona del cuello realizamos medidas del diámetro de dicha región en el mutante phrls y en 
células de tipo silvetre. Tal como muestra

la figura 6 (Chapter I), la longitud del diámetro del cuello en el mutante aumenta aproximadamente 1,5 veces en comparación con el diámetro de la cepa silvetre. Por tanto, Phrlp podría desempeñar un papel en el reforzamiento de la pared celular

\section{Shitf down de pH 7.5 a pH 4.5:}

La expresión de Phr1p en función del pH nos permitió diseñar un experimento en el que pretendíamos analizar si la proteína Phr1p está anclada a la pared celular en algún punto específico. Así, realizamos un experimento en el que tras la incubación a $25^{\circ} \mathrm{C}$ en YPD tamponado a $\mathrm{pH} 7.5, \mathrm{pH}$ en el que la proteína se expresa a altos niveles, pasamos las células a terreno YPD tamponado a $\mathrm{pH}$ 4.5, $\mathrm{pH}$ en el que la expresión de la proteína se reprime. Hipotetizamos que si la proteína deja de expresarse a $\mathrm{pH} 4.5$ la proteina unida a membrana podría sufrir un "turnover" que llevaría a la desaparición de la fluorescencia en torno a la célula mientras que permanecería inalterada en los puntos en los que se encuentre unida covalentemente a la pared celular.

El análisis de los extractos totales de proteína recogidos a diferentes intervalos a partir del paso a YDP pH 4.5 reveló que los niveles de la proteína de fusión Phr1-GFP disminuían rápidamente alcanzando valores del $20 \%$ después de $3 \mathrm{~h}$ y llegando a ser completamente indetectables después de 7h (Figura 7A y B; Chapter I). Dichos resultados sugieren que la disminución del nivel de la proteína de manera tan rápida sea debida a la ausencia de síntesis de novo así como a un alto nivel de degradación de la proteína.

Mediante el análisis microscópico pudimos observar que la fluorescencia verde en torno a la superficie de la célula disminuía de manera rápida. Después de una hora del paso a $\mathrm{pH} \quad 4.5$ la fluorescencia en el contorno de las células había disminuído de manera considerable y llegaba a ser completamente indetectable después de 4.5h. Sin embargo, de manera interesante, la señal verde permanecía intensa en las cicatrices de gemación (Figura 7C; Chapter I). Así, la observación microscópica confirma que la proteína Phr1-GFP presente en la membrana está sujeta a un alto "turnover" y sugiere que la porción de la proteína presente en las cicatrices de gemación o "bud scars" es más estable y está protegida de la degradación. 
Localización de la proteína Phr1 durante la filamentación:

El análisis de la localización de Phr1p durante el crecimiento filamentoso se llevó a cabo usando células de la cepa que expresa la proteína Phr1-GFP. La inducción de la filamentación se llevó a cabo en Medium 199 a pH 7.5 y $37^{\circ} \mathrm{C}$.

Encontramos que la proteína Phr1-GFP a los 30min de la inducción del proceso de filamentación se encuentra altamente concentrada en el ápice de los tubos germinativos. Después de 1h la proteína se encuentra aún presente en el ápice y se hace visible también en las paredes laterales de las hifas. Transcurridas 3h, la señal verde en el ápice deja de ser tan fuerte y la proteína se encuentra distribuída a lo largo de toda la superficie de la hifa sugiriendo una redistribución de la proteína a lo largo de la superficie de la hifa partiendo del ápice. Además Phr1-GFP se encuentra presente también en los septos de las hifas. A tiempos más largos, cuando las hifas son más largas se continua observando dicho patrón de distribución de la fluorescencia (Figure 8; Chapter I).

La localización de Phrlp se analizó con mayor detalle usando un microscopio confocal y utilizando Calcofluor White para determinar si la proteína colocaliza en algún punto con la quitina. Así, pudimos observar que Phr1-GFP se encuentra presente en las regiones apicales de la hifa donde la quitina no se deposita y colocaliza con la quitina a nivel de los septos de las hifas (Figure $9^{a}$ y B; Chapter I).

Dichos resultados indican una clara polarización de la proteína Phrlp durante la filamentación y sugieren un papel crucial de la proteína en el mantenimiento del crecimiento apical necesario para la formación de las hifas.

\section{La polarización de la proteína Phr1- GPF no depende de los microtúbulos:}

Con el objetivo de analizar los mecanismos moleculares mediante los cuales Phr1-GFP localiza de forma polarizada durante el crecimiento filamentoso analizamos la dependencia de los componentes del citoesqueleto.

La dependencia de los microtúbulos fue utilizando Nocodazol (NZ) para inhibir la polimerización de los microtúbulos. La eficacia del inhibitor se analizó mediante coloración de los núcleos con DAPI.

Así, realizamos un experimento en el que se llevó a cabo la inducción del crecimiento filamentoso añadiendo NZ al medio inductor. Con dichos experimentos observamos que la fluorescencia verde se mantenía en el ápicde los germ tubes y en las paredes 
laterales hifas independientemente de la adicción del inhibitor (Figura 10; Chapter I). Sin embargo, observamos una localización defectiva de la proteína Phr1-GFP en los septos de las hifas. La coloración con Calcofluor White nos permitió observar que la quitina se acumulaba de manera aberrante en dichos puntos formando septos engrosados (Figura 11; Chapter I).

Así, la disrupción de la localización de la proteína Phr1-GFP en el ápice de los tubos germinativos o en las paredes laterales de las hifas no depende de los micrútubulos. Sin embargo, localización de la proteína en los septos de las hifas depende de una correcta polimerización de los microtúbulos.

\section{La polarización de la proteína Phr1-} GPF no depende de los microtúbulos:

El requerimiento del citosqueleto de actina se analizó utilizando Citocalasina A (CA) como inhibidor de la formación de los cables de actina. El efecto del inhibidor fue examinado visualizando directamente el citosesqueleto de actina mediante tinción con FaloidinaRodamina.

En un primer experimento la CA fue añadida directamente al medio inductor. De esta manera la formación de hifas queda totalmente inhibida de acuerdo con observaciones previas. Con dicho experimento observamos que la proteína Phr1-GFP da una señal interna y no alcanza la superficie celular de las levaduras. Por tanto, durante el crecimiento en forma de levadura el citosesquelo de actina es necesario para el correcto transporte de la proteína a la superficie celular.

Con el propósito de responder a la pregunta que nos planteábamos sobre la dependencia de la actina para la polarización de Phrlp durante el crecimiento hifal, realizamos un experimento en el que la CA fue añadida 2 h después de la inducción de la filamentación. Con dicho experimento observamos que en las células tratadas con CA la fluorescencia verde en el ápice $\mathrm{y}$ en las paredes laterales de las hifas era mucho menos intensa que en las células control. Además, en muchos casos se observó una acumulación intracelular de la proteína Phr1-GFP. Por tanto, durante la filamentación la actina es necesaria para una correcta localización de la proteína Phr1-GFP siendo necesaria para el transporte polarizado hacia el ápice y para la localización en las paredes laterales de las hifas. 


\section{DISCUSIÓN:}

El trabajo que hemos realizado se centra en la proteína Phr1 de Candida albicans que se sabe desempeña un papel fundamental en la morfogénesis y virulencia del hongo. Sin embargo, no se dispone de información relativa a su localización. La determinación de la localización subcelular de una proteína que contribuye, junto con otro tipo de estudios funcionales a la determinación de su función biológica. Así, en un intento de esclarecer la función de la proteína Phrlp, hemos creado una cepa que expresa una versión funcional de la proteína unida a la Proteína Verde Fluorescente (GFP). El gen utilizado está optimizado para el uso de codones en $C$. albicans.

\section{Localización de la proteína Phr1-GFP en microdominios de membrana:}

En este trabajo la proteína Phrlp ha sido identificada en microdominios de membrana durante el crecimiento vegetativo en Candida albicans. Dicha localización ha sido confirmada mediante estudios bioquímicos en los que Phr1GFP ha sido recupera en la fracción de membrana resistente a detergentes. Precedentemente, análisis mediante SDSPAGE de la fracción rica en proteínas de las membranas resistentes a detergentes (DRMs) en $C$. albicans habían identificado Phrlp como un componente de los "lipid rafts" de membrana (Insenser, Nombela et al. 2006).

Los "lipid rafts" están implicados en multitud de procesos celulares y funcionan como centros de organización para la transducción de señales, para la morfogénesis celular y el mantenimiento de la polaridad celular (Bagnat, Simons, 2002; Alvarez, Douglas, Konopka 2007). Estudios adicionales son necesarios para determinar la presencia de Phrlp en los "lipid rafts" durante el crecimiento filamentoso en C. albicans.

\section{Localización de la proteína Phr1-GFP} durante el crecimiento vegetativo en $C$. albicans:

En células en fase exponencial de crecimiento Phr1-GFP ha sido encontrada en la periferia celular de acuerdo con la localización de membrana de la proteína. Además, encontramos que Phr1-GFP está presente en el anillo de quitina, en el septo y en las cicatrices de gemación colocalizando en dichos puntos con la quitina.

Tanto Gas1p en S. cerevisiae como Phr1p en C. albicans se han considerado durante años como proteína de membrana. Sin embargo, en los últimos años se han mostrado evidencias de la presencia de dichas proteínas en la pared celular (de Groot et al., 2004; De 
Dampaio et. al, 1999; Yin et al., 2005). Además, en esta tesis, en experimentos realizados en paralelo al trabajo en $C$. albicans, hemos demostrado que Gas1p se encuentra covalentemente unida a la pared celular (ver Capítulo III). Así, la colocalización de Phr1p con el anillo de quitina y el septo refuerzan la idea de que estas proteínas desempeñan un papel importante en dichas zonas.

\section{Localización de la proteína Phr1-GFP} durante la filamentación en C. albicans:

El análisis de la localización de la proteína de fusión Phr1-GFP durante la formación de hifas nos ha permitido observar la dinámica de la proteína durante el proceso de filamentación. Phrlp se encuentra concentrada al inicio del proceso en el ápice de los tubos germinativos. A medida que éstos se alargan, la proteína se distribuye gradualmente en las paredes laterales de las hifas y aparece además en los septos donde colocaliza con la quitina. Phr1p se encuentra altamente polarizada durante la filamentación siendo abundante en el ápice y en las regiones apicales de las hifas donde la quitina es menos abundante. Estas observaciones sugieren que Phr1p es necesaria en los puntos de máxima polarización probablemente para la incorporación de nuevos residuos de $\beta$ - (1,3)-glucano en la pared en expansión. Además, la presencia de la proteína a lo largo de la superficie lateral de las hifas podría ayudar a reesforzar la pared celular de las

Dicho patrón de localización durante la filamentación podría ser una consecuencia del transporte de las vesículas de Golgi hacia el ápice de los germ tubes y de las hifas cortas y, más adelante, el transporte de las vesículas podrías presentar un patrón longitudinal a lo largo de las paredes laterales de las hifas. No obstante, dicha distribución de la proteína podría explicarse también como consecuencia de una movilidad lateral de la proteína a través de la bicapa lipídica de la membrana gracias a la presencia del motivo GPI que confiere movilidad a las proteínas. Sin embargo, se ha demostrado que la mayoría del Golgi se distribuye y mantiene en las porciones distales de las hifas cerca del ápice (Rida, Nishikawa et al. 2006). Por tanto, la distribución de Phr1p a lo largo de las hifas parece ser más una consecuencia de la movilidad lateral a través de la membrana dado que la mayor parte del Golgi se concentra en el ápice.

La presencia de Phr1p en el septo de las hifas indica un potencial papel de la proteína también en dicha región. 
Papel del citoesqueleto en la polarización de Phr1p:

Con el objetivo de analizar de manera más detallada los mecanismos moleculares implicados en la polarización de Phrlp durante la filamentación estudiamos el papel de los microtúbulos y el citoesqueleto de actina.

Usando Nocodazol (NZ) como inhibidor de la polimerización de los microtúbulos hemos encontrado que la polarización de Phr1-GFP y su distribución a lo largo de las paredes laterales de las hifas no depende de los microtúbulos. Existen datos publicados en los que se demuestra que el transporte de las vesículas del Golgi no depende de los microtúbulos (Rida, Nishikawa et al. 2006). De manera interesante, encontramos que la localización de la proteína en los septos de las hifas se altera cuando los microtúbulos están depolimerizados. Sin embargo, en este caso debe tenerse en cuenta que la quitina en dichos puntos se encuentra depositada de manera aberrante por lo que la alteración de la localización de Phr1-GFP podría no depender de manera directa de la disfunción de los microtúbulos y estar relacionada con los defectos en la deposición de la quitina.

Respecto al citoesquelo de actina, nuestros datos indican que la polarización y correcta localización de la proteína Phr1 durante la filamentación dependen de una correcta organización del citoesqueleto de actina. En $C$. albicans se ha demostrado que el mantenimiento del Golgi en las regiones distales de las hifas y la movilidad de las vesículas hacia el ápice require un esquelo intacto de actina (Rida, Nishikawa et al. 2006; Veses and Gow 2008). Por tanto, la incorrecta localización de Phr1p cuando el citoesqueleto de actina se encuentra desorganizado podría deberse a la desorganización del Golgi así como a un incorrecto transporte de las vesículas de secrección. 


\section{LA ACTIVIDAD DE LA PROTEÍNA Phr1p ES NECESARIA PARA LOS PROCESOS DE ADHESIÓN E INVASIÓN EN $C$. albicans:}

\section{ANTECEDENTES:}

La capacidad de Candida albicans de responder a cambios en el $\mathrm{pH}$ es fundamental para su supervivencia en distintos nichos ambientales y por tanto también para su potencial como patógeno. Existen distintos genes en $C$. albicans que están regulados por el $\mathrm{pH}$ ambiental a través de la ruta controlada por Rim101 (Bensen, Martin et al. 2004). PHRl es uno de los genes cuya expression está controlada por el $\mathrm{pH}$.

In vivo, el gen PHRl es necesario para la virulencia de $C$. albicans. Usando modelos de infección sistémica en ratones Fonzi y col. demostraron en 1995

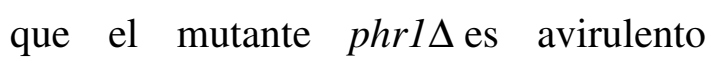
(Ghannoum, Spellberg et al. 1995). En un estudio publicado más tarde se demostró que el fenotipo virulento del mutante nulo de PHRI refleja la dependencia del $\mathrm{pH}$ del defecto morfológico mostrado por el mutante in vitro (De Bernardis, Muhlschlegel et al. 1998). Dado que el pH vaginal es aproximadamente $\quad 4.5$ la capacidad virulenta del mutatne phrls no se encuentra alterada en modelos de infección vaginal en ratas mientras que es avirulento en modelos de infección sistémica dado que el $\mathrm{pH}$ sanguíneo es ligeramente alcalino (alrededor de 7.4).

El primer paso en las interacciones patógeno-hospedador es la union del microorganismo a las superficies epiteliales del hospedador seguido de la invasion a los tejidos internos. Normalmente, la proliferación de los hongos, la colonización y la invasion están dificultadas por densas capas epiteliales con altas tasas de "turnover" y mecanismos de defensa innata tales como la secreción de compuestos antimicrobianos. Sin embargo, $C$. albicans ha desarrollado una serie de mecanismos para superar las barreras defensivas del hospedador tanto para la supervivencia como comensal como para los procesos de infección. Cuando existe un desequilibrio entre las defensas del hospedador y el microorganismo, dichos atributos permiten el desarrollo de infecciones superficiales e incluso la diseminación por el torrente sanguíneo y la invasion de prácticamente todos los órganos del hospedador.

La pared celular es un importante componente en las interacciones patógeno hospedador. $\mathrm{Su}$ esttructura dinámica altera las propiedades físicas de la interfase hongo-hospedador y por tanto 
influencia el reconocimiento por parte de los componentes del sistema immune del hospedador y además es determinante en los procesos de adhesión e invasión.

\section{OBJETIVOS:}

Con este trabajo pretendiamos obtener un mayor entendimiento del papel de la proteína Phrlp en la patogénesis de Candida albicans. Así, nos propusimos investigar si el mutante phrls era defectuoso en los estadíos iniciales de la interacción patógeno hospedador. En particular, nuestros objetivos fueron: analizar la capacidad del mutante phrls (1) de adhesion tanto a superficies epiteliales como a superficies abióticas y (2) de invasión usando modelos de epitelio humano reconstituído (RHE).

Los experimentos pertinentes fueron realizados durante mi estancia en el "Fraunhofer Institut" en Stuttgart (Germany) donde trabajé con el grupo del Dr. Steffen Rupp.

\section{RESULTADOS:}

El mutante phr1 $\operatorname{ces}$ defectuoso en su capacidad de adhesion a superficies abióticas:

La capacidad de adhesión del mutante phrl $\Delta$ se analizó utilizando un modelo de adhesión que ha sido puesto a punto por y descrito por Rupp et al. (Sohn, Senyurek et al. 2006). En dicho modelo los ensayos de adhesión se llevan a cabo en placas de poliestireno de 24 pocillos que en el caso de la adhesión a superficies abióticas se usan sin previo tratamiento. En dichas placas se efectúa la infección con las células de $C$. albicans y se incuban a $37^{\circ} \mathrm{C}$ bajo atmósfera de $\mathrm{CO}_{2}(5 \%)$ usando D-MEM suplementado con suero bovino fetal (10\%) y a distintos intervalos desde el momento de la infección las células adherents y no-adherentes se plaquean en YPD sólido. Después de tres días se procede al recuento de colonias (cfu) para después obterner el porcentaje de células adherentes.

En nuestros experimentos, las células de $C$. albicans fueron previamente incubadas en YDP tamponado a $\mathrm{pH} 6$ para evitar la influencia de $P H R 2$, homólogo de PHRl.

En lo que respecta a la adhesión a la superficie plástica observamos que el mutante phrls presenta una marcada 
reducción en la adhesión en comparación con la cepa silvestre. El defecto en adhesión se hacía visible desde los 30 cuando la cepa silvestre alcanzaba valores de aproximadamente el $80 \%$ y los valores del mutante eran del $20 \%$. Con el transcurso del experimento la cepa silvestre alcanzaba valores máximos del $95 \%$ mientras que los valores máximos del mutante phrls eran del $30 \%$. En los experimentos se incluyó además la cepa reconstituida en la que se introdujo uno alelos del gen PHRl. La cepa reconstituida presentó los mismos valores que los de la cepa silvestre (Figura 2A, Chapter II).

Además, como control adicional realizamos el mismo experimento pero tamponando el terreno (D-MEM) a $\mathrm{pH}$ 6.0 que es el valor de $\mathrm{pH}$ en el que el

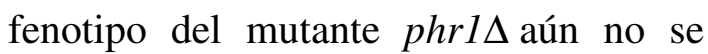
manifiesta y la expresión del homólogo PHR2 es mínima. Con dicho experimento no se encontraron diferencias significativas entre el mutante y la cepa silvestre indicando que el defecto en adhesión está específicamente asociado a la deleción de PHRl (Figura 2A, Chapter II).
La pérdida de Phr1p reduce la adhesión a líneas celulares epiteliales:

Con el objetivo de analizar la capacidad del mutante phrls de adherirse a los epitelios humanos se realizó un ensayo similar al anterior en el que la superficie de los pocillos de las placas (24-well-plates) habían sido previamente tratados con líneas celulares epiteliales hasta crear una monocapa confluente. Utilizamos dos líneas celulares: Caco2, una línea celular del epitelio intestinal y TR146, una línea celular de epitelio oral. El pH del medio utilizado era 7.5, pH óptimo para el crecimiento y mantenimiento de las líneas celulares. Dicha condición era apropiada para analizar el comportamiento del mutante $p h r l \Delta$ dado que a $\mathrm{pH} 7.5$ el gen $P H R I$ se expresa a altos niveles.

Los resultados obtenidos con las dos líneas celulares utilizadas fueron similares. En ambos casos la cepa silvestre presentó una cinética de adhesión más lenta y menos eficiente de la que presentó en adhesión a la plástica. Dichas observaciones concuerdan con datos en los que se demuestra que la adhesión a la plástica es más eficiente que la adhesión a líneas celulares epiteliales (Sohn, Senyurek et al. 2006). No obstante, la cepa silvestre alcanzó porcentajes de adhesión después de $4 \mathrm{~h}$ 
del $95 \%$ mientras que los valores máximos alcanzados por el mutante no fueron mayores del 30\%. Los valores de adhesión de la cepa reconstituida revertieron los valores de la cepa silvestre.

La expresión de genes específicos de hifas y adhesinas se encuentra en el mutante phr1s durante la adhesión a una superficie abiótica:

Mediante experimentos de RT-PCR cuantitativa se analizó la expresión de una serie de genes durante la condición de adhesión al poliestireno.

Analizamos la expresión de genes que codifica una adhesinas, ALS3 and EAP1. $A L S 3$ es un miembro de la Familia genes Als ("agglutinin-like genes") que codifican glicoproteínas implicadas en adhesión Chaffin, 2008). ALS3 se expresa de manera específica en la fase hifal. EAPl es un gen que se sabe está implicado en la unión a poliestireno y a células epiteliales humanas ( $\mathrm{Li}$ et al., 2007). Además, analizamos la expresión de dos genes específicos de hifas, HWP1 y ECE1. HWP1 codifica una proteína de superficie celular que se actúa como target de transglutaminasas de mamífero (Chaffin, 2008). ECEl se encuentra altamente expresado durante el crecimiento hifal y su expresión incrementa a medida que aumenta la longitud de las hifas (Birse, Irwin et al. 1993; Chaffin, 2008).

Tal como muestra la Figura 4 (Chapter II), los niveles transcripcionales de $A L S 3$ y EAP1 no se encontraron afectados en el mutante phrls mostrando aproximadamente los mismos niveles que los de la cepa silvestre. Dado que $A L S 3$ es un gen específico de hifas su regulacion en la condición de adhesión en el mutante puede darse debido a una activación del programa hifal en el mutante. De manera interesante, la deleción del gen PHRI causa una marcada reducción en los niveles transcripcionales de $H W P 1$ y ECE1 que sufren una reducción en el mutante del 60 y $65 \%$ respectivamente con respecto a la cepa silvestre. Sin embargo, dicha downregulación se encontró también en células en suspensión (datos no mostrados).

\section{El mutante $p h r 1 \Delta$ es tiene afectada su capacidad de invasión a epitelio humano reconstituído:}

La capacidad de invasión del mutante phrls se analizó in vitro utilizando un modelo de tejido humano reconstituido (RHE) desarrollado en el laboratorio de Rupp S. (Hernandez and Rupp 2009). 
Dicho modelo consiste en la preparación de insertos que contienen una monocapa de células de la linea celular Caco2 sobre una matriz de colágeno. Los insertos así preparados son infectados con las células de Candida albicans, colocados en pocillos contenientes D-MEM suplementado con suero bovino fetal (10\% FCS) e incubados a $37^{\circ} \mathrm{C}$ bajo atmósfera de $\mathrm{CO}_{2}$. Después de 7 y $24 \mathrm{~h}$ los insertos son procesados mediantes técnicas rutinarias de histología (la Figura 1 de Materials and Methods muestra un esquema del experimento).

En nuestros experimentos, las células de C. albicans fueron previamente incubadas en YDP tamponado a pH 6.0.

Como se observa en la Figura 5, después de 24 h la cepa silvestre había penetrado a través de la capa de células epiteliales invadiendo la matriz de colágeno. Por su parte, el mutante phrl $\Delta$ mostró claros defectos tanto en la penetración en el epitelio como en la invasión de la matriz de colágeno. La cepa reconstituida fue capaz de penetrar en la capa de epitelio e invadir la matriz de colágeno aunque se encontraron efectos de dosis génica. Las morfologías celulares de las distintas cepas fueron analizadas en detalle a las 7 y las $24 \mathrm{~h}$. Tanto la cepa silvestre como la cepa reconstituida formaron hifas que llegaban a ser muy alargadas a las $24 \mathrm{~h}$. La cepa mutante no fue capaz de filamentar y se observó la morfología que ha sido descrita durante la filamentación en medio líquido con tubos germinativos distorsionados que no llegan a alargarse.

\section{DISCUSIÓN:}

Adhesión e invasión son dos procesos fundamentals para el establecimiento de las infecciones por Candida. Los resultados de este trabajo indican que en el mutante phrls de C. albicans la capacidad de adhesión tanto a susperficies plásticas como a epitelio humano reconstituído se encuentran claramente alteradas. Además hemos analizado la capacidad de invasión del mutante nulo de PHRI utilizando un modelo de tejido humano reconstituído. Hemos observado que el mutante es completamente defectivo en su capacidad de penetrar a través del epitelio e invadir el espacio subyacente.

Los defectos combinados de adhesión e invasión in vitro presentados aquí probablemente contribuyan a la avirulencia del mutante phrls encontrada en modelos de infección sistémica (De Bernardis et al., 1998). De acuerdo con un papel crucial del gen PHRl en adhesión e invasión, recientemente $P H R 1$ ha sido encontrado como uno de los genes inducidos de 
manera significativa en un modelo de infección oral (Wilson et al., 2009).

En un intento de las analizar las bases moleculares del defecto en adhesión del mutante phrls, hemos analizado la expresión de una serie de genes que codifican proteínas unidas a GPI. En particular se analizó la expresión de $A L S 3$ y $E A P 1$, dos genes que codifican adhesinas, y de HWPl y ECEl que son dos genes que se expresan de manera diferencial en las hifas.

No encontramos diferencias en la expresión de $A L S 3$ ni de EAPl en el mutante phrls con respecto a la cepa silvestre. Dado que ALS3 es un gen que se expresa diferencialment en la condicion de filamentación, su inducción durante la adhesión en el mutante parece indicar que una inducción del programa de inducción hifal en el mutante a pesar de la incapacidad del mismo de formar hifas. Sin embargo, los defectos en el ensamblaje de la pared celular debidos a la ausencia de Phr1p en el mutante pueden afectar la localización de la proteína codifica por ALS3. Recientemente se ha demostrado que anticuerpos monoclonas anti-Als3 pueden bloquear la adhesión en $\mathrm{C}$. albicans sugiriendo que la exposición de determinados epítopos de la proteína es crucial para el proceso de achesión. (Coleman, Oh et al. 2009). Por tanto, aunque no hemos encontrado diferencias ne la expresión del gen, no podemos excluir un papel de la proteína Als3p en la adhesión dado que la proteína podría encontrarse "enmascarada" debido a los defectos de pared celular del mutante phrls. Los mismo podría ocurrir también con la proteína Eap1p. Phr1p, a través de su actividad glucanosiltransferásica, crea sitios de union para otras manoproteínas (Fonzi 1999). En ausencia de la actividad de Phrlp la unión y localización específica de manoproteínas de superficie podría estar alterada $\mathrm{y}$, en consecuencia, las propiedades de adhesión se podrían encontrar afectadas.

Por su parte, los genes HWP1 y ECE1 se encontraron fuertemente reprimidos en la cepa delecionada en PHRI con respecto a la cepa silvestre. Sin embargo dichos genes se encontraron también reprimidos en células en suspension en condiciones de indución de la filamentación (datos no mostrados). Por tanto, la repression de dichos genes parece no ser específica de la condición de adhesión. Debe tenerse en cuenta que Phr1p, ha sido identificada como un componente del proteoma de la pared celular de C. albicans (de Groot et al., 2004). Una fración de la proteína unida a membrane mediante GPI podría sufrir una reacción de transglicosilación en la 
superficie celular y encontrarse anclada

al glucano de pared celular, como ocurre en otras manoproteínas (De Groot et al., 2005). Así, Phr1p podría estar expuesta a la superficie celular y su actividad podría afectar a la localización de otras manoproteínas. 


\section{LA IMBOLIZACIÓN DE LA PROTEÍNA Gas1p DE $S$. cerevisiae EN EL ANILLO DE CHITINA ES ESENCIAL PARA UNA CORRECTA MORFOGÉNESIS:}

\section{ANTECEDENTES:}

Los miembros de la familia Gas de Saccharomyces cerevisiae son enzimas cuya actividad es importante para el remodelamiento de la pared celular (Popolo and Vai 1999; Mouyna, Fontaine et al. 2000). Gas1p es el miembro major caracterizado de la famila. Las células de los mutantes delecionados en el gen GAS1 presentan, además de una reducida tasa de crecimiento, defectos morfológicos, mostrándose como células redondeadas y de gran tamaño. Los mutantes gas $1 \Delta$, sin embargo, previenen la lisis celular causada por el debilitamiento de la pared celular mediante la activación de una respuesta compensatoria en la que se inducen varios genes y se producen cambios en la composición y arquitectura de la pared celular (Popolo, Vai et al. 1993; Popolo, Ragni et al 2001; Lagorce, Hause et al 2003).

GAS1 se expresa durante el crecimiento vegetativo junto con GAS5, que parece tener un papel auxiliar, y
$G A S 3$, que se expresa a bajos liveles y su función es desconocida.

La proteína Gas1p, al igual que el resto de proteínas Gas, presenta una molécula glicolipídica, un glicosilfosfatidilinositol (GPI), en el extremo carboxi-terminal. Se sabe que el motivo GPI sirve como anclaje a la parte externa de la membrane plasmática y confiere movilidad en el ambiente lipídico de la membrana a las proteíonas que lo contienen (Malinska, Malinsky et al. 2004). Además, la presencia del GPI promueve la asociación con microdominios específicos de la membrana enriquecidos en esfingolípidos y ergosterol que han sido denominados "lipid rafts". Dichos microdominios de membrana se caracterizan por ser insolubles en detergents no-iónicos, tales como Triton X100 (TX100) a bajas temperaturas La proteína Gas1p fue el primer miembro de la familia en ser recuperada en análisis bioquímicos en las membranas resistentes a los detergents (DRMs, del ingles "detergent-resistan membranes") (Bagnat, Keranen et al. 2000). Más recientemente Gas3p y Gas5p han sido también recuperadas en las DRMs (Aronova, Wedaman et al. 2007).

Debe tenerse en cuenta un posible anclaje de la proteína Gas1p a la pared celular que fue propuesto por primera 
vez en 1999 cuando se demostró que la proteína se liberaba de la pared celular con tratamiento con una $\beta-(1,3)$ glucanasa (De Sampaio, Bourdineaud et al. 1999). Posteriormente análisis de espectrometría de masas han revelado que Gas1p, Gas3p y Gas5p se encuentran covalentemente unidas al complejo de glucanos de la pared celular (Yin, de Groot et al. 2005).

\section{OBJETIVOS:}

El objetivo de esta parte del trabajo fue el estudio de la localización de la proteína Gas1p en $S$. cerevisiae utilizanto técnicas de fusión con proteínas fluorescentes. En particular, nuestro objetivo fue el análisis de la localización subcelular de Gas1p durante el crecimiento vegetativo $y$ en condiciones de crecimiento polarizado.

Además, con este trabajo pretendíamos también estudiar si el anclaje de Gas1p a la pared celular que había sido previamente observado se debía a una anomalía en el mecanismo de retención a la membrana plasmática o si, por el contrario, la pared celular es un destino específico de la proteína.

\section{RESULTADOS:}

Construcción de una versión functional de la proteína de fusión mRFP-Gas1:

Para la obtención de la proteína de fusión se realizó un tagging interno de la proteína Gas1p con la proteína de fluorescencia roja mRFP (monomeric Red Fluorescent Protein) en el extremo amino amino de la proteína. En particular la mRFP se insertó detrás de la sequencia señal (esquema en Figura 1A; Chapter I). Utilizando el plásmido pMF608 que contiene la cassetta mRFP-GAS1 con el promotor natural de GAS1 realizamos una transformación integrativa. Para ello, en primer lugar procedimos a la linearización del plásmido con la enzima BfrI. Los transformantes Leu+ así obtenidos se analizaron para la presencia de la proteína de fusión. De manera interesante, encontramos que la proteína de fusión mRFP-GAS1 sólo se producía y secretaba de manera correcta cuando se expresaba en una cepa mutante de gas $1 \Delta$.

Para determinar si la proteína de fusión así creada era functional analizamos su capacidad de suprimir el defecto fenotípico del mutante gas $1 \Delta$. Mediante un test de sensibildad a Calcofluor White pudimos observar que la cepa expresando la proteína de fusión complementa de manera total la hipersensibilidad a Calcofluor White del 
mutante gas $1 \Delta$. También se encontró que revertía los defectos morfológicos de las células del mutante gas $1 \Delta$ (Figura 1B y C; Chapter I). Además, la cepa que expresaba la proteína de fusión revertía los valores de la reducida tasa de crecimiento de la cepa gasld a los valores de la cepa silvestre.

\section{Localización de la proteína Gas1 durante el crecimiento vegetativo:}

La observación microscópica nos permitió determinar la localización de la proteína mRFP-Gas1p durante el crecimiento vegetativo. En células en fase exponencial de crecimiento la proteína mRFP-Gas1p está presente en diversos puntos. La proteína de fusión se encuentra presente en la periferia celular de acuerdo con la localización de la proteína en la membrana plasmática. Además, en células en división se encuentra en dos puntos brillantes que se localizan a ambos lados del cuello entre la célula madre e hija y en una fase más tardía de la división la fluorescencia roja decora completamente la región del septo. Se encontró también una fluorescencia interna en algunas estructuras con forma de cráter que podrían corresponder con las cicatrices de gemación o "bud-scars" que se forman después de la citoquinesis. Incluso, en algunas células encontramos segmentos brillantes que podrían corresponder con los "bud-scars" en una visión transversal (Figure 2, Chapter I).

En el laboratorio se han obtenido resultados similares respecto a la localización de Gas1 durante el crecimiento vegetativo usando una fusión con la Proteína Verde Fluorescente (GFP). La inserción de la proteína GFP se realizó en la región carboxi-terminal de la proteína, concretamente en una región rica en serinas delante de la secuencia señal para el anclaje al GPI. Además, en este caso, el gen de fusión no se integró en el genoma sino que se colocó en plásmidos episómicos. Cuando la dicha proteína de fusión se expresó en tanto en un mutante gas $1 \Delta$ como en la cepa parental, ambos con un background genético diverso al utilizado en la fusión con la mRFP, la proteína de fusión se encontró en las mismas cuatro localizaciones mencionadas anteriormente. La proteína estaba presente en la membrana plasmática, en el cuello entre la célula madre e hija, en la región del septo y en la cicatriz de gemación o "bud scar" (Figure 3; Chapter I). De esta manera pudimos confirmar que, independientemente del tipo de proteína fluorescente, el sitio de fusión, backgraound genético o dosis 
génica, la proteína Gas1p presenta el mismo patrón de localización.

\section{La proteína de fusión mRFP-Gas1 se localiza en dos "pools": uno móvil y otro inmóvil:}

Con el objetivo de analizar si la localización de Gas1p cambia en condiciones de crecimiento polarizado diseñamos un experimento en el que las células de la cepa que expresa la proteína de fusión mRFP-Gas1 fueron tratadas con $\alpha$-Factor $(\alpha-F)$. $\alpha-F$ es una feromona que arresta las células en fase G1 y después de $3 \mathrm{~h}$ se observa la formación de "shmoos". El efecto de la feromona en la progresión del ciclo celular se analizó mediante el cálculo de la percentual de gemación de las células después a distintos intervalos después de la adición del $\alpha-F$. En células tratadas con $\alpha-F$ encontramos una marcada reducción en el índice de gemación y además se observó la formación de estructuras tipo "shmoo". Mediante observación microscópica pudimos observar que la adición del $\alpha-F$ conducía a una rápida disminución de la fluorescencia roja en el contorno de la proteína. La señal que se observaba en la superficie celular se hacía más débil con el transcurso del experimento y después de $3,25 \mathrm{~h}$ la señal en el contorno celular era prácticamente indetectable. Sin embargo, de manera interesante, la fluorescencia roja continuaba siendo intensa en las cicatrices de gemación ("bud scars”) y en algunos septos. Dichas observaciones sugerían que la proteína de fusión mRFP-Gas1 está sujeta a un alto "turnover" en la membrana plasmática mientras que la asociación de la proteína con las cicatrices de gemación podría protegerla de la degradación.

Para confirmar dicha hipótesis procedimos a determinar los niveles de de proteína endógena y proteína de fusión en lisados totales a distintos intervalos después de la adición del $\alpha-F$. Así, mediante análisis por Western Blot pudimos observar que los niveles relativos de la proteína endógena durante el tratamiento con $\alpha-\mathrm{F}$ permanecían prácticamente constantes mientras que los niveles de la proteína de fusión mRFP-Gas1p disminuían de manera drástica con el tratamiento con $\alpha-F$. Durante la obtención de los extractos de proteína total las paredes celulares habían sido descartadas.

Dado que durante el trataminto con el $\alpha-\mathrm{F}$ GAS1 no se expresa y los niveles del $\mathrm{ARN}$ mensjero disminuyen rápidamente, estas observaciones sugieren que la tasa de degradacion de la proteína mRFP- 
Gas1p es mayor que la de la protéina endógena. Por tanto, la disminución de la fluorescencia observada al microscopio probablemente se debe a un alto "turnover" así como a la ausencia de síntesis de novo de la proteína.

La localización de la proteína se analizó con más detalle mediante microscopía confocal realizando además una tinción de la quitina con Calcofluor White. Así, pudimos observar que la fluorescencia que permanence en los "bud scar" después del tratamiento con $\alpha-F$ colocaliza con la quitina.

\section{La forma inmóvil de Gas1p se} encuentra unida covalentemente a la pared celular en los "bud scars":

El particular comportamiento de la proteína de fusión Gas1 con la mRFP en el extremo amino nos permitió investigar más detalladamente la localización de la proteína en los "bud scars" o cicatrices de gemación. Así, realizamos un análisis mediante Western Blot de las diferentes fracciones subcelulares y de las paredes celulares purficadas obtenidas antes de la adicción de la adicción del $\alpha$-F y después de 3,25h, momento en el que los liveles de proteína total habían sido prácticmente indetectables. Como puede observarse en el immunoblot de la Figura 5 (Chapter I), en células no tratadas tanto la proteína endógena como la proteína de fusión mRFP-Gas1p se detectaron en la fracción de membrana. Sin embargo, en células tratadas con $\alpha-F$ Gas1p se encontró enriquecida en la fracción de membrana mientras que la proteína mRFP-Gas1 no se detectó ni en la fracción de membrana ni en la fracción total. Además, tanto Gas1p como mRFPGas1p se detectaron en el material liberado después del tratamiento de las paredes celulares purificadas con una $\beta$ (1,3)-glucanasa. Dicho resultado concuerda con los datos publicados del análisis del proteoma de $S$. cerevisiae en en los que Gas1p se ha detectado en la pared celular (Yin, de Groot et al. 2005).

\section{DISCUSIÓN:}

En este trabajo hemos investigado la localización de Gas1p mediante el uso de técnicas de fusión de proteínas y nuestro conocimeitno sobre los dominios estructurales de la proteína. La construcción de una version functional de la proteína mRFP-Gas1 mediante un tagging interno en la región amino terminal nos permitió el análisis de la localización de Gas1p en S. cerevisiae. 
Localización de la proteína Gas1 en Saccharomyces cerevisiae durante el crecimiento vegetativo:

Durante el crecimiento vegetativo mRFP-Gas1p se encontró en distintas localizaciones. La proteína de fusión se encontró decorando la membrana plasmática en consistencia con la localización de membrane de la proteína. Además, la proteina se detectó en el anillo de quitina, en la región del septo y en las cicatrices de gemación donde permanecía durante varias generaciones.

Resultados similares se obtuvieron en el laboratorio utilizando una fusión con la Proteína Verde Fluorescente (GFP) en la región carboxi-terminal de la proteína.

Utilizando ambas proteínas de fusión observamos que la proteína Gas1p presenta la misma localización independientemente del tipo de proteína fluorescente utilizada, del sitio de inserción, background genético o dosis génica.

\section{Identificación de una fracción de la proteína mRFP-Gas1 que se encuentra unida covalentemente a la pared celular:}

La presencia de Gas1p en la pared celular se describió por primera vez en un estudio en el que la proteína se recuperó mediante tratamiento de la pared celular con una $\beta$-(1,3)-glucanasa (De Sampaio, Bourdineaud et al. 1999). Gas1p, junto con Gas3p y Gas5p, fue posteriormente identificada, como covalentemente unida a la pared (Yin, de Groot et al. 2005). Un estudio cuantitativo identificó la presencia de un número pequeño de moléculas de Gas1p en la pared celular (Yin, de Groot et al. 2007) indicando que la pared celular no es la localización principal de la proteína. $\mathrm{Su}$ detección en la pared celular ha generado controversia. La presencia de Gas1p en el proteoma de pared celular podría deberse a un alto nivel de expresión que podría causar un mal funcionamiento del mecanismo de retención de la proteína a la membrana plasmática así como a la alta sensibilidad de la técnica de espectrometría de masas. Una hipótesis alternativa es que Gas1p se distribuya entre la membrana plasmática y la pared celular debido a la presencia de un amplio dominio rico en serinas (Ser-box) antes del sitio de $\omega$ que puede afectar a la distribución de las proteínas unidas a GPI entre la membrana y la pared celular (Frieman and Cormack 2004).

En este trabajo hemos validado la hipótesis de la existencia de una fracción de Gas1p que se encuentra unida covalentemente a la pared celular. La proteína mRFP-Gas1 ha sido encontrada 
en dos fracciones: una fracción móvil localizada en la membrana plasmática y una fracción inmóvil que se encuentra unida de manera covalente a la pared celular donde se encuentra estabilizada. Además, aprovechamos la inestabilidad de mRFP-Gas1p para analizar la localización de la forma de Gas1p unida de manera específica a las cicatrices de gemación. En células tratadas con $\alpha$ Factor, una feromona que arresta las células en $\mathrm{G} 1$, observamos una rápida degradación de la proteína mRFP-Gas1 así como una desaparición de la fluorescencia en el contorno de las células. Sin embargo, observamos que la proteína permanecía unida a las cicatrices de gemación (o "bud scars"). En análisis mediante Western Blot de fracciones subcelulares de células tratadas con $\alpha-F$ la proteína mRFP-Gas1p no se detectó ni en las fracción de membrane ni en la fracción de proteína total mientras que Gas1p se encontraba enriquecida en la fracción de membrana. Además, tanto Gas1p como mRFP-Gas1p se detectaron en el material liberado por tratamiento enzimático con $\beta$-(1,3)-glucanase indicando que la molécula aceptora en la union a la quitina de Gas1p en la cicatriz de gemación es el $\beta$-(1,3)-glucano. Análisis bioquímicos adicionales en los que se ha analizado con detalle la naturaleza del anclaje se han llevado a cabo en el labotorio (Rolli, Ragni et al., 2009).

La incorporación de proteínas en la pared celular de una manera localizada ha sido demonstrada para las manoproteínas Tip1p, Cw1p y Cwp2p. La incorporación de Tip1p, Cw2p en la pared celular se ha demostrado que depende del momento de la transcriptición durante el ciclo celular. La localización de $\mathrm{Cw} 1 \mathrm{p}$ depende no solo de su expresión durante el ciclo celular, sino también del momento de formación del septo y de la composición del mismo (Smits, Schenkman et al. 2006). La síntesis de Gas1p se ha encontrado que es maxima durante la transición G1 a $\mathrm{S}$ coincidiendo con el momento de formación del anillo de quitina (Popolo, Cavadini et al. 1993; Ram, Brekelmans et al. 1995). La incorporación de Gas1p en el anillo de quitina podría estar determinada por dicho patrón de expresión aunque no se pueden excluir otro tipo de mecanismos. 
Los resultados aquí presentados sobre la localización de la proteína mRFP-Gas1p durante el crecimiento vegetativo, junto con otros datos obtenidos en el laboratorio, han sido publicados recientemente en un artículo (Rolli E., 2009). En dicho trabajo hemos demostrado que la proteína Gas1p se encuentra en diversas localizaciones durante el crecimiento vegetativo. Gas1p localiza en la membrana celular, en el anillo de quintina y en el septo y permanence, después de la citoquinesis, en la cicatriz de gemación o "bud scar”. Además, nuestros experimentos han demostrado que la inmovilización de Gas1p en el anillo de chitina y en el septo es fundamental para la morfogénesis.

Immobilization of the Glycosylphosphatidylinositol-anchored Gas1 Protein into the Chitin Ring and Septum is required for proper morphogenesis in Yeast.

Rolli E, Ragni E, Calderon J, Porello S, Fascio U, Popolo L. Mol Biol Cell. November 19, 2009

(Artículo adjunto al final del capítulo III- Annex Chapter III) 


\section{CONCLUSIONES GENERALES:}

\section{En Candida albicans:}

1. La proteína Phr1-GFP localiza en puntos discretos de la superficie celular encontrándose en los "lípid rafts" de membrana durante el crecimiento vegetativo

2. La proteína Phr1p se localiza en la periferia celular, en la región del cuello entre madre e hija, en el septo y en la cicatriz de gemación durante el crecimiento vegetativo

3. Phrlp es necesaria para reforzar la región del cuello durante la división celular

4. La proteína Phr1-GFP se concentra en el ápice de los tubos germinativos al inicio de la filamentación. Cuando los tubos germinativos se alargan la proteína permanece en la zona apical de las hifas y se distribuye gradualmente a lo largo de las paredes laterales de las hifas

5. La proteína Phrlp se encuentra altamente polarizada durante la filamentación. Phr1p es necesaria en los puntos de máximo crecimiento probablemente debido su función en la incorporación y alargamiento del $\beta$-(1,3)-glucano recién sintetizado de la pared celular en expansión

6. La localización de la proteína Phr1p en el ápice de los tubos germinativos y en las paredes laterales de las hifas no depende de los microtúbulos mientras que es necesaria una correcta polimerización de los mismos para la correcta localización de la proteína en los septos de las hifas

7. En células levaduriformes el citoesqueleto de actina es necesario para un correcto transporte de la proteína Phr1-GFP a la superficie celular y durante la formación de hifas la actina es necesaria para la localización polarizada de la proteína

8. El mutante $p h r 1 \Delta$ presenta defectos en la adhesión a superficies abióticas

9. La pérdida de la proteína Phr1p reduce notablemente la adhesión de las células de C. albicans a células epiteliales 
10. La expression de los genes $A L S 3$ y EAPl no se encuentra afectada en el mutante phrl $\Delta$ con respecto a la cepa Silvestre durante la adhesión a superficies abióticas. Por su parte, los niveles de expresión de los genes HWPl y ECE1 se encuentran marcadamente reducidos como consecuencia de la delección del gen PRH1. Sin embargo, la subexpresión de los genes HWP1 y ECE1 no es específica de la condición de adhesión

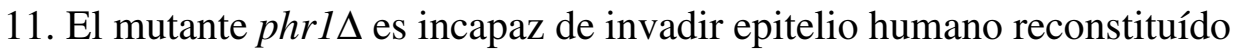

En Saccharomyces cerevisiae:

5. La proteína Gas 1p se localiza en la periferia celular, en el anillo de quitina y en el septo y después de la citoquinesis permanece en las cicatrices de gemación

6. La presencia de la proteína Gas1p en el anillo de quitina es necesaria para mantener la rigidez en la región del cuello

7. La proteína mRFP-Gas1 se encuentra presente en dos fracciones: una móvil que se localiza en la membrane plasmática y una inmóvil que se encuentra protegida de degradación

8. La fracción inmóvil de la proteína Gas1p se encuentra unida covalentemente a la pared celular 
Abe, M., I. Nishida, et al. (2001). "Yeast 1,3-beta-glucan synthase activity is inhibited by phytosphingosine localized to the endoplasmic reticulum." J Biol Chem 276(29): 26923-30.

Akashi, T., T. Kanbe, et al. (1994). "The role of the cytoskeleton in the polarized growth of the germ tube in Candida albicans." Microbiology 140 ( Pt 2): 27180.

Almirante, B., D. Rodriguez, et al. (2005). "Epidemiology and predictors of mortality in cases of Candida bloodstream infection: results from population-based surveillance, barcelona, Spain, from 2002 to 2003." $\underline{\mathrm{J} \text { Clin }}$ Microbiol 43(4): 1829-35.

Alvarez, F.J., Douglas, L. M., et al (2007) " Sterol-rich plasma membrane domains in fungi" Eukaryotic Cell. 6(5):755-63

Aronova, S., K. Wedaman, et al. (2007). "Probing the membrane environment of the TOR kinases reveals functional interactions between TORC1, actin, and membrane trafficking in Saccharomyces cerevisiae." $\underline{\mathrm{Mol}}$ Biol Cell 18(8): 2779-94.

Arroyo, J., J. Sarfati, et al. (2007). "The GPI-anchored Gas and Crh families are fungal antigens." Yeast 24(4): 289-96.

Asmundsdottir, L. R., H. Erlendsdottir, et al. (2008). "Molecular epidemiology of candidemia: evidence of clusters of smoldering nosocomial infections." Clin Infect Dis 47(2): e17-24.

Au-Young, J. and P. W. Robbins (1990). "Isolation of a chitin synthase gene (CHS1) from Candida albicans by expression in Saccharomyces cerevisiae." $\underline{\mathrm{Mol}}$ Microbiol 4(2): 197-207.

Baek, Y. U., S. J. Martin, et al. (2006). "Evidence for novel $\mathrm{pH}$ - dependent regulation of Candida albicans Rim101, a direct transcriptional repressor of the cell wall beta-glycosidase Phr2." Eukaryot Cell 5(9): 1550-9.

Bagnat, M., S. Keranen, et al. (2000). "Lipid rafts function in biosynthetic delivery of proteins to the cell surface in yeast." Proc Natl Acad Sci U S A 97(7): 3254-9.

Bagnat, M. and K. Simons (2002). "Cell surface polarization during yeast mating." Proc Natl Acad Sci U S A 99(22): 14183-8.

Bagnat, M. and K. Simons (2002). "Lipid rafts in protein sorting and cell polarity in budding yeast Saccharomyces cerevisiae." Biol Chem 383(10): 1475-80.

Bennett, R. J. and A. D. Johnson (2005). "Mating in Candida albicans and the search for a sexual cycle." Annu Rev Microbiol 59: 233-55.

Bensen, E. S., S. J. Martin, et al. (2004). "Transcriptional profiling in Candida albicans reveals new adaptive responses to extracellular $\mathrm{pH}$ and functions for Rim101p." Mol Microbiol 54(5): 1335-51.

Berman, J. and P. E. Sudbery (2002). "Candida Albicans: a molecular revolution built on lessons from budding yeast." Nat Rev Genet 3(12): 918-30.

Birse, C. E., M. Y. Irwin, et al. (1993). "Cloning and characterization of ECE1, a gene expressed in association with cell elongation of the dimorphic pathogen Candida albicans." Infect Immun 61(9): 3648-55.

Biswas, S., P. Van Dijck, et al. (2007). "Environmental sensing and signal transduction pathways regulating morphopathogenic determinants of Candida albicans." Microbiol Mol Biol Rev 71(2): 348-76. 
Boone, C., A. Sdicu, et al. (1991). "Isolation from Candida albicans of a functional homolog of the Saccharomyces cerevisiae KRE1 gene, which is involved in cell wall beta-glucan synthesis." $\mathrm{J}$ Bacteriol 173(21): 6859-64.

Brown, A. J., F. C. Odds, et al. (2007). "Infection-related gene expression in Candida albicans." Curr Opin Microbiol 10(4): 30713.

Brown, D. A. and E. London (1998) "Functions of lípid rafts in biological membranas" Annu Rev Cell Dev Biol. 14:111-36.

Brown, J. L. and H. Bussey (1993). "The yeast KRE9 gene encodes an $\mathrm{O}$ glycoprotein involved in cell surface beta-glucan assembly." Mol Cell Biol 13(10): 6346-56.

Bulawa, C. E. (1993). "Genetics and molecular biology of chitin synthesis in fungi." Annu Rev Microbiol 47: 505-34.

Bulawa, C. E., D. W. Miller, et al. (1995). "Attenuated virulence of chitin-deficient mutants of Candida albicans." Proc Natl Acad Sci U S A 92(23): 10570-4.

Cabib E. (2004) "The septation apparatus, a chitin-requiring machine in pudding yeast" Arch Biochem Biophys. 426(2):201-7.

Cabib, E., N. Blanco, et al. (2007). "Crh1p and Crh2p are required for the cross-linking of chitin to beta(1-6)glucan in the Saccharomyces cerevisiae cell wall." Mol Microbiol 63(3): 92135.

Cabib, E., T. Drgon, et al. (1997). "The yeast cell wall, a dynamic structure engaged in growth and morphogenesis." Biochem Soc Trans 25(1): 200-4.

Cabib, E., V. Farkas, et al. (2008). "Assembly of the yeast cell wall. Crh1p and Crh2p act as transglycosylases in vivo and in vitro." J Biol Chem 283(44): 29859-72.

Cabib, E., D. H. Roh, et al. (2001). "The yeast cell wall and septum as paradigms of cell growth and morphogenesis." J Biol Chem 276(23): 19679-82.

Cabib, E., A. Sburlati, et al. (1989). "Chitin synthase 1, an auxiliary enzyme for chitin synthesis in Saccharomyces cerevisiae." $\underline{\mathbf{J}}$ Cell Biol 108(5): 1665-72.

Calderone, R. A. and W. A. Fonzi (2001). "Virulence factors of Candida albicans." $\underline{\text { Trends }}$ Microbiol 9(7): 327-35.

Caro, L. H., H. Tettelin, et al. (1997). "In silicio identification of glycosylphosphatidylinositol-anchored plasma-membrane and cell wall proteins of Saccharomyces cerevisiae." Yeast 13(15): 147789.

Carotti, C., E. Ragni, et al. (2004). "Characterization of recombinant forms of the yeast Gas1 protein and identification of residues essential for glucanosyltransferase activity and folding." Eur J Biochem 271(18): 3635-45.

Castillon, G. A., N. R. Adames, et al. (2003). "Septins have a dual role in controlling mitotic exit in budding yeast." Curr Biol 13(8): 654-8.

Cid, V. J., A. Duran, et al. (1995). "Molecular basis of cell integrity and morphogenesis in Saccharomyces cerevisiae." Microbiol Rev 59(3): 345-86.

Coleman, D. A., S. H. Oh, et al. (2009). "Monoclonal antibodies specific for Candida albicans Als3 that immunolabel fungal cells in vitro and in vivo and block adhesion to host surfaces." J Microbiol Methods 78(1): 71-8.

Conzelmann, A., C. Fankhauser, et al. (1991). "Biosynthesis of glycophosphoinositol anchors in 
Saccharomyces cerevisiae." Cell Biol Int Rep 15(9): 863-73.

Cote, P., H. Hogues, et al. (2009). "Transcriptional Analysis of the Candida albicans Cell Cycle." Mol Biol Cell.

Crampin, H., K. Finley, et al. (2005). "Candida albicans hyphae have a Spitzenkorper that is distinct from the polarisome found in yeast and pseudohyphae." J Cell Sci 118(Pt 13): 2935-47.

Chaffin, W. L. (2008). "Candida albicans cell wall proteins." Microbiol Mol Biol Rev 72(3): 495-544.

Chaffin, W. L., J. L. Lopez-Ribot, et al. (1998). "Cell wall and secreted proteins of Candida albicans: identification, function, and expression." Microbiol Mol Biol Rev 62(1): 130-80.

Chandra, J., D. M. Kuhn, et al. (2001). "Biofilm formation by the fungal pathogen Candida albicans: development, architecture, and drug resistance." $\mathrm{J}$ Bacteriol 183(18): 5385-94.

Chen-Wu, J. L., J. Zwicker, et al. (1992). "Expression of chitin synthase genes during yeast and hyphal growth phases of Candida albicans." Mol Microbiol 6(4): 497-502.

Choi, W. J., A. Sburlati, et al. (1994). "Chitin synthase 3 from yeast has zymogenic properties that depend on both the CAL1 and the CAL3 genes." Proc Natl Acad Sci U S A 91(11): 4727-30.

d'Enfert, C., S. Goyard, et al. (2005). "CandidaDB: a genome database for Candida albicans pathogenomics." Nucleic Acids Res 33(Database issue): D353-7.

Davis, D. (2003). "Adaptation to environmental $\mathrm{pH}$ in Candida albicans and its relation to pathogenesis." Curr Genet 44(1): $1-7$.
Davis, D., J. E. Edwards, Jr., et al. (2000). "Candida albicans RIM101 pH response pathway is required for host-pathogen interactions." Infect Immun 68(10): 5953-9.

Davis, D., R. B. Wilson, et al. (2000). "RIM101-dependent andindependent pathways govern $\mathrm{pH}$ responses in Candida albicans." Mol Cell Biol 20(3): 971-8.

De Bernardis, F., F. A. Muhlschlegel, et al. (1998). "The $\mathrm{pH}$ of the host niche controls gene expression in and virulence of Candida albicans." Infect Immun 66(7): 3317-25.

de Groot, P. W., A. D. de Boer, et al. (2004). "Proteomic analysis of Candida albicans cell walls reveals covalently bound carbohydrate-active enzymes and adhesins." Eukaryot Cell 3(4): 955-65.

De Groot, P. W., A. F. Ram, et al. (2005). "Features and functions of covalently linked proteins in fungal cell walls." Fungal Genet Biol 42(8): 657-75.

Delgado, N., Xue, J. et al (2003) "A recombinant beta-1,3glucanosyltransfersae homologo of Coccidioides posadasii protects mice aginst coccidioidomycosis" Infect Immun. 71(6):3010-9

De Sampaio, G., J. P. Bourdineaud, et al. (1999). "A constitutive role for GPI anchors in Saccharomyces cerevisiae: cell wall targeting." Mol Microbiol 34(2): 247-56.

Desnos-Ollivier, M., S. Bretagne, et al. (2008). "Mutations in the fks 1 gene in Candida albicans, C. tropicalis, and C. krusei correlate with elevated caspofungin MICs uncovered in AM3 medium using the method of the European Committee on Antibiotic Susceptibility Testing." 
Antimicrob Agents Chemother 52(9): 3092-8.

Dijkgraaf, G. J., M. Abe, et al. (2002). "Mutations in Fks1p affect the cell wall content of beta-1,3- and beta-1,6-glucan in Saccharomyces cerevisiae." Yeast 19(8): 671-90.

Douglas, C. M. (2001). "Fungal beta(1,3)-D-glucan synthesis." Med Mycol 39 Suppl 1: 55-66.

Douglas, C. M., J. A. D'Ippolito, et al. (1997). "Identification of the FKS1 gene of Candida albicans as the essential target of 1,3-betaD-glucan synthase inhibitors." Antimicrob Agents Chemother 41(11): 2471-9.

Eckert, S. E., W. J. Heinz, et al. (2007). "PGA4, a GAS homologue from Candida albicans, is up-regulated early in infection processes." Fungal Genet Biol 44(5): 368-77.

Ernst, J. F. (2000). "Transcription factors in Candida albicans environmental control of morphogenesis." Microbiology 146 ( Pt 8): 1763-74.

Filler, S. G. and D. C. Sheppard (2006). "Fungal invasion of normally non-phagocytic host cells." PLoS Pathog 2(12): e129.

Finley, K. R. and J. Berman (2005). "Microtubules in Candida albicans hyphae drive nuclear dynamics and connect cell cycle progression to morphogenesis." Eukaryot Cell 4(10): 1697-711.

Firon, A., G. Lesage, et al. (2004). "Integrative studies put cell wall synthesis on the yeast functional map." Curr Opin Microbiol 7(6): 617-23.

Fluckiger, U., O. Marchetti, et al. (2006). "Treatment options of invasive fungal infections in adults." Swiss Med Wkly 136(29-30): 447-63.

Fonzi, W. A. (1999). "PHR1 and PHR2 of Candida albicans encode putative glycosidases required for proper cross-linking of beta-1,3and beta-1,6-glucans." Bacteriol 181(22): 7070-9.

Ford, R. A., J. A. Shaw, et al. (1996). "Yeast chitin synthases 1 and 2 consist of a non-homologous and dispensable $\mathrm{N}$-terminal region and of a homologous moiety essential for function." Mol Gen Genet 252(4): 420-8.

Frieman, M. B. and B. P. Cormack (2003). "The omega-site sequence of glycosylphosphatidylinositolanchored proteins in Saccharomyces cerevisiae can determine distribution between the membrane and the cell wall." Mol Microbiol 50(3): 883-96.

Frieman, M. B. and B. P. Cormack (2004). "Multiple sequence signals determine the distribution of glycosylphosphatidylinositol proteins between the plasma membrane and cell wall in Saccharomyces cerevisiae." Microbiology 150(Pt 10): 310514.

Fujita, M., O. T. Yoko, et al. (2006). "Inositol deacylation by Bst1p is required for the quality control of glycosylphosphatidylinositolanchored proteins." Mol Biol Cell 17(2): 834-50.

Futai, E., T. Maeda, et al. (1999). "The protease activity of a calpain-like cysteine protease in Saccharomyces cerevisiae is required for alkaline adaptation and sporulation." Mol Gen Genet 260(6): 559-68.

Garcia, R., C. Bermejo, et al. (2004). "The global transcriptional response to transient cell wall damage in Saccharomyces cerevisiae and its regulation by the cell integrity signaling pathway." J Biol Chem 279(15): 15183-95.

Gatti, E., L. Popolo, et al. (1994). "Olinked oligosaccharides in yeast 
glycosyl phosphatidylinositolanchored protein gp115 are clustered in a serine-rich region not essential for its function." J Biol Chem 269(31): 19695-700.

Gauwerky, K., C. Borelli, et al. (2009). "Targeting virulence: a new paradigm for antifungals." Drug Discov Today 14(3-4): 214-22.

Ghannoum, M. A., B. Spellberg, et al. (1995). "Reduced virulence of Candida albicans PHR1 mutants." Infect Immun 63(11): 4528-30.

Gietz, R. D., R. H. Schiestl, et al. (1995). "Studies on the transformation of intact yeast cells by the LiAc/SSDNA/PEG procedure." Yeast 11(4): $355-60$.

Gladfelter, A. S., J. R. Pringle, et al. (2001). "The septin cortex at the yeast mother-bud neck." $\underline{\text { Curr }}$ Opin Microbiol 4(6): 681-9.

Gow, N. A., P. W. Robbins, et al. (1994). "A hyphal-specific chitin synthase gene (CHS2) is not essential for growth, dimorphism, or virulence of Candida albicans." Proc Natl Acad Sci U S A 91(13): 6216-20.

Gozalbo, D., P. Roig, et al. (2004). "Candida and candidiasis: the cell wall as a potential molecular target for antifungal therapy." Curr Drug Targets Infect Disord 4(2): 117-35.

Hausler, A., L. Ballou, et al. (1992). "Yeast glycoprotein biosynthesis: MNT1 encodes an alpha-1,2mannosyltransferase involved in O-glycosylation." Proc Natl Acad Sci U S A 89(15): 6846-50.

Hazan, I., M. Sepulveda-Becerra, et al. (2002). "Hyphal elongation is regulated independently of cell cycle in Candida albicans." $\mathrm{Mol}$ Biol Cell 13(1): 134-45.

Hernandez, R. and S. Rupp (2009). "Human epithelial model systems for the study of Candida infections in vitro: part II.
Histologic methods for studying fungal invasion." Methods Mol Biol 470: 105-23.

Herrero, A. B., P. Magnelli, et al. (2004). "KRE5 gene null mutant strains of Candida albicans are avirulent and have altered cell wall composition and hypha formation properties." Eukaryot Cell 3(6): 1423-32.

Horn, D. L., D. Neofytos, et al. (2009). "Epidemiology and outcomes of candidemia in 2019 patients: data from the prospective antifungal therapy alliance registry." $\underline{\text { Clin }}$ Infect Dis 48(12): 1695-703.

Huh, W. K., J. V. Falvo, et al. (2003). "Global analysis of protein localization in budding yeast." Nature 425(6959): 686-91.

Hurtado-Guerrero, R., A. W. Schuttelkopf, et al. (2009). "Molecular mechanisms of yeast cell wall glucan remodeling." $\underline{\mathbf{J}}$ Biol Chem 284(13): 8461-9.

Iida, H., Y. Yagawa, et al. (1990). "Essential role for induced $\mathrm{Ca} 2+$ influx followed by $[\mathrm{Ca} 2+] \mathrm{i}$ rise in maintaining viability of yeast cells late in the mating pheromone response pathway. A study of $[\mathrm{Ca} 2+] \mathrm{i}$ in single Saccharomyces cerevisiae cells with imaging of fura-2." J Biol Chem 265(22): 13391-9.

Inoue, S. B., N. Takewaki, et al. (1995). "Characterization and gene cloning of 1,3-beta-D-glucan synthase from Saccharomyces cerevisiae." Eur J Biochem 231(3): 845-54.

Insenser, M., C. Nombela, et al. (2006). "Proteomic analysis of detergentresistant membranes from Candida albicans." Proteomics 6 Suppl 1: S74-81.

Jaafar, L., I. Moukadiri, et al. (2003). "Characterization of a disulphide-bound Pir-cell wall protein (Pir-CWP) of Yarrowia lipolytica." Yeast 20(5): 417-26. 
Jones, T., N. A. Federspiel, et al. (2004).

"The diploid genome sequence of Candida albicans." Proc Natl Acad Sci U S A 101(19): 732934.

Kapteyn, J. C., R. C. Montijn, et al. (1996). "Retention of Saccharomyces cerevisiae cell wall proteins through a phosphodiester-linked beta-1,3/beta-1,6-glucan heteropolymer." Glycobiology 6(3): 337-45.

Kapteyn, J. C., A. F. Ram, et al. (1997). "Altered extent of cross-linking of beta1,6-glucosylated mannoproteins to chitin in Saccharomyces cerevisiae mutants with reduced cell wall beta1,3-glucan content." $\quad \underline{\mathbf{J}}$ Bacteriol 179(20): 6279-84.

Kapteyn, J. C., P. Van Egmond, et al. (1999). "The contribution of the O-glycosylated protein Pir2p/Hsp150 to the construction of the yeast cell wall in wild-type cells and beta 1,6-glucandeficient mutants." Microbiol 31(6): 1835-44.

Karkowska-Kuleta, J., M. Rapala-Kozik, et al. (2009). "Fungi pathogenic to humans: molecular bases of virulence of Candida albicans, Cryptococcus neoformans and Aspergillus fumigatus." Biochim Pol.

Kitamura, A., K. Someya, et al. (2009). "Discovery of a small-molecule inhibitor of \{beta\}-1,6-glucan synthesis." Antimicrob Agents Chemother 53(2): 670-7.

Klebl, F. and W. Tanner (1989). "Molecular cloning of a cell wall exo-beta-1,3-glucanase from Saccharomyces cerevisiae." $\quad \underline{J}$ Bacteriol 171(11): 6259-64.

Klis, F. M., P. Mol, et al. (2002). "Dynamics of cell wall structure in Saccharomyces cerevisiae." FEMS Microbiol Rev 26(3): 23956.
Kollar, R., E. Petrakova, et al. (1995). "Architecture of the yeast cell wall. The linkage between chitin and beta(1-->3)-glucan." J Biol Chem 270(3): 1170-8.

Kollar, R., B. B. Reinhold, et al. (1997). "Architecture of the yeast cell wall. Beta(1-->6)-glucan interconnects mannoprotein, beta(1-->)3-glucan, and chitin." Biol Chem 272(28): 17762-75.

Kullas, A. L., S. J. Martin, et al. (2007). "Adaptation to environmental $\mathrm{pH}$ : integrating the Rim101 and calcineurin signal transduction pathways." Mol Microbiol 66(4): 858-71.

Lagorce, A., N. C. Hauser, et al. (2003). "Genome-wide analysis of the response to cell wall mutations in the yeast Saccharomyces cerevisiae." $\mathrm{J}$ Biol Chem 278(22): 20345-57.

Lamb, T. M. and A. P. Mitchell (2003). "The transcription factor Rim101p governs ion tolerance and cell differentiation by direct repression of the regulatory genes NRG1 and SMP1 in Saccharomyces cerevisiae." $\mathrm{Mol}$ Cell Biol 23(2): 677-86.

Lane, S., C. Birse, et al. (2001). "DNA array studies demonstrate convergent regulation of virulence factors by $\mathrm{Cph} 1, \mathrm{Cph} 2$, and Efg1 in Candida albicans." J Biol Chem 276(52): 48988-96.

Lesage, G. and H. Bussey (2006). "Cell wall assembly in Saccharomyces cerevisiae." Microbiol Mol Biol Rev 70(2): 317-43.

Levin, D. E. (2005). "Cell wall integrity signaling in Saccharomyces cerevisiae." Microbiol Mol Biol Rev 69(2): 262-91.

Li, M., S. J. Martin, et al. (2004). "Candida albicans Rim13p, a protease required for Rim101p processing at acidic and alkaline pHs." Eukaryot Cell 3(3): 74151. 
Liu, H. (2001). "Transcriptional control of dimorphism in Candida albicans." Curr Opin Microbiol 4(6): 728-35.

Lo, H. J., J. R. Kohler, et al. (1997). "Nonfilamentous C. albicans mutants are avirulent." Cell 90(5): 939-49.

Lussier, M., A. M. Sdicu, et al. (1995). "Localization and targeting of the Saccharomyces cerevisiae Kre2p/Mnt1p alpha 1,2mannosyltransferase to a medialGolgi compartment." J Cell Biol 131(4): 913-27.

Lussier, M., A. M. Sdicu, et al. (1998). "The Candida albicans KRE9 gene is required for cell wall beta-1, 6-glucan synthesis and is essential for growth on glucose." Proc Natl Acad Sci U S A 95(17): 9825-30.

Malinska, K., J. Malinsky, et al. (2003). "Visualization of protein compartmentation within the plasma membrane of living yeast cells." Mol Biol Cell 14(11): 4427-36

Malinska, K., J. Malinsky, et al. (2004). "Distribution of Can1p into stable domains reflects lateral protein segregation within the plasma membrane of living S. cerevisiae cells." J Cell Sci 117(Pt 25): 6031-41.

Martin, S. W. and J. B. Konopka (2004). "Lipid raft polarization contributes to hyphal growth in Candida albicans." Eukaryot Cell 3(3): 675-84.

Mavor, A. L., S. Thewes, et al. (2005). "Systemic fungal infections caused by Candida species: epidemiology, infection process and virulence attributes." Curr Drug Targets 6(8): 863-74.

Meaden, P., K. Hill, et al. (1990). "The yeast KRE5 gene encodes a probable endoplasmic reticulum protein required for (1----6)-betaD-glucan synthesis and normal cell growth." Mol Cell Biol 10(6): 3013-9.

Metcalf, S. C. and D. H. Dockrell (2007) "Improve outcomes associated with advances in therapy for invasive fangal infections in immunocompromised hosts" Infect. 55(4):287-99

Metcalf, S. C. and D. H. Dockrell (2007) "Improve outcomes associated with advances in therapy for invasive fangal infections in immunocompromised hosts" $\underline{\mathrm{J}}$ Infect. 55(4):287-99

Mio, T., T. Yabe, et al. (1996). "Role of three chitin synthase genes in the growth of Candida albicans." J Bacteriol 178(8): 2416-9.

Mio, T., T. Yamada-Okabe, et al. (1997). "Isolation of the Candida albicans homologs of Saccharomyces cerevisiae KRE6 and SKN1: expression and physiological function." $\quad \underline{\mathrm{J}}$ Bacteriol 179(7): 2363-72.

Moukadiri, I., L. Jaafar, et al. (1999). "Identification of two mannoproteins released from cell walls of a Saccharomyces cerevisiae mnn1 mnn9 double mutant by reducing agents." $\underline{\mathrm{J}}$ Bacteriol 181(16): 4741-5.

Mouyna, I., T. Fontaine, et al. (2000). "Glycosylphosphatidylinositolanchored glucanosyltransferases play an active role in the biosynthesis of the fungal cell wall." J Biol Chem 275(20): 14882-9.

Mouyna, I., W. Morelle, et al. (2005). "Deletion of GEL2 encoding for a beta(1-3)glucanosyltransferase affects morphogenesis and virulence in Aspergillus fumigatus." Mol Microbiol 56(6): 1675-88.

Muhlschlegel, F. A. and W. A. Fonzi (1997). "PHR2 of Candida albicans encodes a functional 
homolog of the pH-regulated gene PHR1 with an inverted pattern of $\mathrm{pH}$-dependent expression." Mol Cell Biol 17(10): 5960-7.

Munro, C. A., D. A. Schofield, et al. (1998). "Regulation of chitin synthesis during dimorphic growth of Candida albicans." Microbiology 144 ( Pt 2): 391401.

Munro, C. A., K. Winter, et al. (2001). "Chs1 of Candida albicans is an essential chitin synthase required for synthesis of the septum and for cell integrity." Mol Microbiol 39(5): 1414-26.

Naglik, J., A. Albrecht, et al. (2004). "Candida albicans proteinases and host/pathogen interactions." Cell Microbiol 6(10): 915-26.

Naglik, J. R., S. J. Challacombe, et al. (2003). "Candida albicans secreted aspartyl proteinases in virulence and pathogenesis." Microbiol Mol Biol Rev 67(3): 400-28, table of contents.

Nather, K. and C. A. Munro (2008). "Generating cell surface diversity in Candida albicans and other fungal pathogens." Microbiol Lett 285(2): 137-45.

Oh, S. H., G. Cheng, et al. (2005). "Functional specificity of Candida albicans Als3p proteins and clade specificity of ALS3 alleles discriminated by the number of copies of the tandem repeat sequence in the central domain." Microbiology 151(Pt 3): 673-81.

Onishi, J., M. Meinz, et al. (2000). "Discovery of novel antifungal (1,3)-beta-D-glucan synthase inhibitors." Antimicrob Agents Chemother 44(2): 368-77.

Orlean, P. (1987). "Two chitin synthases in Saccharomyces cerevisiae." J Biol Chem 262(12): 5732-9.

Orlean, P., H. Ammer, et al. (1986). "Synthesis of an O-glycosylated cell surface protein induced in yeast by alpha factor." Proc Natl Acad Sci U S A 83(17): 6263 6266.

Papaleo, E., P. Fantucci, et al. (2006). "Three-dimensional structure of the catalytic domain of the yeast beta-(1,3)-glucan transferase Gas1: a molecular modeling investigation." $\mathrm{J}$ Mol Model 12(2): 237-48.

Phan, Q. T., C. L. Myers, et al. (2007). "Als3 is a Candida albicans invasin that binds to cadherins and induces endocytosis by host cells." PLoS Biol 5(3): e64.

Popolo, L., P. Cavadini, et al. (1993). "Transcript accumulation of the GGP1 gene, encoding a yeast GPI-anchored glycoprotein, is inhibited during arrest in the G1 phase and during sporulation." Curr Genet 24(5): 382-7.

Popolo, L., D. Gilardelli, et al. (1997). "Increase in chitin as an essential response to defects in assembly of cell wall polymers in the ggp1delta mutant of Saccharomyces cerevisiae." $\underline{\mathrm{J}}$ Bacteriol 179(2): 463-9.

Popolo, L., R. Grandori, et al. (1988). "Immunochemical characterization of gp115, a yeast glycoprotein modulated by the cell cycle." Eur J Cell Biol 47(2): 173-80.

Popolo, L., Ragni, E. et al. (2001). "The yeast cell-wall salvage pathway" Med Mycol. 39(1):111-21.

Popolo, L., E. Ragni, et al. (2008). "Disulfide bond structure and domain organization of yeast beta(1,3)-glucanosyltransferases involved in cell wall biogenesis." J Biol Chem 283(27): 18553-65.

Popolo, L. and M. Vai (1998). "Defects in assembly of the extracellular matrix are responsible for altered morphogenesis of a Candida albicans phr1 mutant." Bacteriol 180(1): 163-6. 
Popolo, L. and M. Vai (1999). "The Gas1 glycoprotein, a putative wall polymer cross-linker." Biochim Biophys Acta 1426(2): 385-400.

Popolo, L., M. Vai, et al. (1993). "Physiological analysis of mutants indicates involvement of the Saccharomyces cerevisiae GPI-anchored protein gp115 in morphogenesis and cell separation." I Bacteriol 175(7): 1879-85.

Porta, A., A. M. Ramon, et al. (1999). "PRR1, a homolog of Aspergillus nidulans palF, controls $\mathrm{pH}$ dependent gene expression and filamentation in Candida albicans." J Bacteriol 181(24): 7516-23.

Pruyne, D., A. Legesse-Miller, et al. (2004). "Mechanisms of polarized growth and organelle segregation in yeast." Annu Rev Cell Dev Biol 20: 559-91.

Ragni, E., A. Coluccio, et al. (2007). "GAS2 and GAS4, a pair of developmentally regulated genes required for spore wall assembly in Saccharomyces cerevisiae." Eukaryot Cell 6(2): 302-16.

Ragni, E., T. Fontaine, et al. (2007). "The Gas family of proteins of Saccharomyces cerevisiae: characterization and evolutionary analysis." Yeast 24(4): 297-308.

Ram, A. F., S. S. Brekelmans, et al. (1995). "Identification of two cell cycle regulated genes affecting the beta 1,3-glucan content of cell walls in Saccharomyces cerevisiae." FEBS Lett 358(2): 165-70.

Ram, A. F., A. Wolters, et al. (1994). "A new approach for isolating cell wall mutants in Saccharomyces cerevisiae by screening for hypersensitivity to calcofluor white." Yeast 10(8): 1019-30.

Rambourg, A., C. L. Jackson, et al. (2001). "Three dimensional configuration of the secretory pathway and segregation of secretion granules in the yeast Saccharomyces cerevisiae." $\quad \underline{J}$ Cell Sci 114(Pt 12): 2231-9.

Ramon, A. M. and W. A. Fonzi (2003). "Diverged binding specificity of Rim101p, the Candida albicans ortholog of PacC." Eukaryot Cell 2(4): 718-28.

Raudaskoski, M., Mao, W. Z. et al (1994) "Microtubule cytoskeleton in hyphal growth. Response to nocodazole in a sensitive and a tolerante strain of the homobasidiomycete Shizophyllum commune" Eur J Cell Biol. 64 (1):131-41

Reyes, A., M. Sanz, et al. (2007). "Chitin synthase III requires Chs4pdependent translocation of Chs3p into the plasma membrane." J Cell Sci 120(Pt 12): 1998-2009.

Richardson, M. and R. Rautemaa (2009). "How the host fights against Candida infections." Front Biosci (Schol Ed) 1: 246-57.

Richardson, M. D. (2005). "Changing patterns and trends in systemic fungal infections." $\mathrm{J}$ Antimicrob Chemother 56 Suppl 1: i5-i11.

Rida, P. C., A. Nishikawa, et al. (2006). "Yeast-to-hyphal transition triggers formin-dependent Golgi localization to the growing tip in Candida albicans." Mol Biol Cell 17(10): 4364-78.

Roberts, R. L., B. Bowers, et al. (1983). "Chitin synthesis and localization in cell division cycle mutants of Saccharomyces cerevisiae." $\mathrm{Mol}$ Cell Biol 3(5): 922-30.

Rodriguez-Pena, J. M., C. Rodriguez, et al. (2002). "Mechanisms for targeting of the Saccharomyces cerevisiae GPI-anchored cell wall protein $\mathrm{Crh} 2 \mathrm{p}$ to polarised growth sites." J Cell Sci 115(Pt 12): 2549-58.

Roemer, T. and H. Bussey (1991). "Yeast beta-glucan synthesis: 
KRE6 encodes a predicted type II membrane protein required for glucan synthesis in vivo and for glucan synthase activity in vitro." Proc Natl Acad Sci U S A 88(24): 11295-9.

Roemer, T. and H. Bussey (1995). "Yeast Kre1p is a cell surface Oglycoprotein." Mol Gen Genet 249(2): 209-16.

Roemer, T., S. Delaney, et al. (1993). "SKN1 and KRE6 define a pair of functional homologs encoding putative membrane proteins involved in beta-glucan synthesis." Mol Cell Biol 13(7): 4039-48.

Roemer, T., B. Jiang, et al. (2003). "Large-scale essential gene identification in Candida albicans and applications to antifungal drug discovery." Mol Microbiol 50(1): 167-81.

Roh, D. H., B. Bowers, et al. (2002). "The septation apparatus, an autonomous system in budding yeast." Mol Biol Cell 13(8): 2747-59.

Rolli, E., Ragni, E. (2009) "Immobilization of the Glycosylphosphatidylinositolanchored Gas1 Protein into the Chitin Ring and Septum Is Required for Proper Morphogenesis in Yeast" $\mathrm{Mol}$ Biol Cell. Sep 30. [Epub ahead of print]

Romani, L., F. Bistoni, et al. (2003). "Adaptation of Candida albicans to the host environment: the role of morphogenesis in virulence and survival in mammalian hosts." Curr Opin Microbiol 6(4): $338-43$.

Roumanie, O., C. Weinachter, et al. (2001). "Functional characterization of the Bag7, Lrg1 and Rgd2 RhoGAP proteins from Saccharomyces cerevisiae." FEBS Lett 506(2): 149-56.
Rupp, S. (2007). "Interactions of the fungal pathogen Candida albicans with the host." Future Microbiol 2: 141-51.

Sanchez-Martinez, C. and J. PerezMartin (2001). "Dimorphism in fungal pathogens: Candida albicans and Ustilago maydis-similar inputs, different outputs." Curr Opin Microbiol 4(2): 21421.

Saporito-Irwin, S. M., C. E. Birse, et al. (1995). "PHR1, a pH-regulated gene of Candida albicans, is required for morphogenesis." Mol Cell Biol 15(2): 601-13.

Schaller, M., C. Borelli, et al. (2005). "Hydrolytic enzymes as virulence factors of Candida albicans." Mycoses 48(6): 36577.

Selvaggini, S., C. A. Munro, et al. (2004). "Independent regulation of chitin synthase and chitinase activity in Candida albicans and Saccharomyces cerevisiae." Microbiology 150(Pt 4): 921-8.

Shahinian, S. and H. Bussey (2000). "beta-1,6-Glucan synthesis in Saccharomyces cerevisiae." $\underline{\mathrm{Mol}}$ Microbiol 35(3): 477-89.

Sharkey, L. L., M. D. McNemar, et al. (1999). "HWP1 functions in the morphological development of Candida albicans downstream of EFG1, TUP1, and RBF1." Bacteriol 181(17): 5273-9.

Shaw, J. A., P. C. Mol, et al. (1991). "The function of chitin synthases 2 and 3 in the Saccharomyces cerevisiae cell cycle." J Cell Biol 114(1): 111-23.

Silverman, S. J., A. Sburlati, et al. (1988). "Chitin synthase 2 is essential for septum formation and cell division in Saccharomyces cerevisiae." Proc Natl Acad Sci U S A 85(13): 4735-9.

Smits, G. J., L. R. Schenkman, et al. (2006). "Role of cell cycle- 
regulated expression in the localized incorporation of cell wall proteins in yeast." Mol Biol Cell 17(7): 3267-80.

Sohn, K., I. Senyurek, et al. (2006). "An in vitro assay to study the transcriptional response during adherence of Candida albicans to different human epithelia." FEMS Yeast Res 6(7): 1085-93.

Soll, D. R. (2004). "Mating-type locus homozygosis, phenotypic switching and mating: a unique sequence of dependencies in Candida albicans." Bioessays 26(1): 10-20.

Staib, P., M. Kretschmar, et al. (2000). "Differential activation of a Candida albicans virulence gene family during infection." Proc Natl Acad Sci U S A 97(11): 6102-7.

Staib, P. and J. Morschhauser (2007). "Chlamydospore formation in Candida albicans and Candida dubliniensis--an enigmatic developmental programme." Mycoses 50(1): 1-12.

Steinberg, G., Wedlich-Söldner, R., et al (2001). "Microtubules in the fangal pathogen Ustilago maydis are highly dynamic and determine cell polarity" $\mathrm{J}$ Cell Sci. 114(3):609-22.

Sudbery, P., N. Gow, et al. (2004). "The distinct morphogenic states of Candida albicans." $\underline{\text { Trends }}$ Microbiol 12(7): 317-24.

Sudoh, M., S. Nagahashi, et al. (1993). "Cloning of the chitin synthase 3 gene from Candida albicans and its expression during yeasthyphal transition." Mol Gen Genet 241(3-4): 351-8.

Thewes, S., M. Kretschmar, et al. (2007). "In vivo and ex vivo comparative transcriptional profiling of invasive and noninvasive Candida albicans isolates identifies genes associated with tissue invasion." Mol Microbiol 63(6): 1606-28.

Trautwein, M., C. Schindler, et al. (2006). "Arf1p, Chs5p and the ChAPs are required for export of specialized cargo from the Golgi." EMBO J 25(5): 943-54.

Trilla, J. A., T. Cos, et al. (1997). "Characterization of CHS4 (CAL2), a gene of Saccharomyces cerevisiae involved in chitin biosynthesis and allelic to SKT5 and CSD4." Yeast 13(9): 795-807.

Tsuchimori, N., L. L. Sharkey, et al. (2000). "Reduced virulence of HWP1-deficient mutants of Candida albicans and their interactions with host cells." Infect Immun 68(4): 1997-2002.

Urban, C., K. Sohn, et al. (2003). "Identification of cell surface determinants in Candida albicans reveals Tsa1p, a protein differentially localized in the cell." FEBS Lett 544(1-3): 22835.

Valdez-Taubas, J. and H. R. Pelham (2003). "Slow diffusion of proteins in the yeast plasma membrane allows polarity to be maintained by endocytic cycling." Curr Biol 13(18): 163640.

Valdivieso, M. H., L. Ferrario, et al. (2000). "Chitin synthesis in a gas1 mutant of Saccharomyces cerevisiae." J Bacteriol 182(17): 4752-7.

Verstrepen, K. J. and F. M. Klis (2006). "Flocculation, adhesion and biofilm formation in yeasts." $\underline{\mathrm{Mol}}$ Microbiol 60(1): 5-15.

Veses, V. and N. A. Gow (2008). "Vacuolar dynamics during the morphogenetic transition in Candida albicans." FEMS Yeast Res 8(8): 1339-48.

Walker, L. A., Gow, N. A. et al. (2009) "Fungal echinocandin resistance" 
Fungal Genet Biol. 2009 Sep 19. [Epub ahead of print]

Warenda, A. J. and J. B. Konopka (2002). "Septin function in Candida albicans morphogenesis." Mol Biol Cell 13(8): 2732-46.

Weissman, Z. and D. Kornitzer (2004). "A family of Candida cell surface haem-binding proteins involved in haemin and haemoglobin-iron utilization." Mol Microbiol 53(4): 1209-20.

Whiteway, M. and U. Oberholzer (2004). "Candida morphogenesis and host-pathogen interactions." Curr Opin Microbiol 7(4): 350-7.

Wilson, D., S. Thewes, et al. (2009). "Identifying infection-associated genes of Candida albicans in the postgenomic era." FEMS Yeast Res.

Xue, J., Hung, C. Y. et al. (2005). "Immune response of vaccinated and non-vaccinated mice to Coccidioides posadasii infection" Vaccine. 23(27):3535-44

Yang, Y. L. (2003). "Virulence factors of Candida species." J Microbiol Immunol Infect 36(4): 223-8.

Yin, Q. Y., P. W. de Groot, et al. (2007). "Mass spectrometric quantitation of covalently bound cell wall proteins in Saccharomyces cerevisiae." FEMS Yeast Res 7(6): 887-96.

Yin, Q. Y., P. W. de Groot, et al. (2005). "Comprehensive proteomic analysis of Saccharomyces cerevisiae cell walls: identification of proteins covalently attached via glycosylphosphatidylinositol remnants or mild alkali-sensitive linkages." J Biol Chem 280(21): 20894-901.

Yokoyama, K., H. Kaji, et al. (1990). "The role of microfilaments and microtubules in apical growth and dimorphism of Candida albicans." $\mathrm{J}$ Gen Microbiol 136(6): 1067-75.

Zakikhany, K., S. Thewes, et al. (2008). "From attachment to invasion: infection associated genes of Candida albicans." Nippon Ishinkin Gakkai Zasshi 49(4): 245-51. 